\title{
Study of B Meson Decays to ppbarh Final States
}

\author{
Tetiana B. Hryn’ova \\ Stanford Linear Accelerator Center \\ Stanford University \\ Stanford, CA 94309
}

SLAC-Report-810

Prepared for the Department of Energy

under contract number DE-AC02-76SF00515

Printed in the United States of America. Available from the National Technical Information Service, U.S. Department of Commerce, 5285 Port Royal Road, Springfield, VA 22161. 
This document, and the material and data contained therein, was developed under sponsorship of the United States Government. Neither the United States nor the Department of Energy, nor the Leland Stanford Junior University, nor their employees, nor their respective contractors, subcontractors, or their employees, makes an warranty, express or implied, or assumes any liability of responsibility for accuracy, completeness or usefulness of any information, apparatus, product or process disclosed, or represents that its use will not infringe privately owned rights. Mention of any product, its manufacturer, or suppliers shall not, nor is it intended to, imply approval, disapproval, or fitness of any particular use. A royalty-free, nonexclusive right to use and disseminate same of whatsoever, is expressly reserved to the United States and the University. 
STUDY OF $B$ MESON DECAYS TO $p \bar{p} h$ FINAL STATES

\author{
A DISSERTATION \\ SUBMITTED TO THE DEPARTMENT OF PHYSICS \\ AND THE COMMITTEE ON GRADUATE STUDIES \\ OF STANFORD UNIVERSITY \\ IN PARTIAL FULFILLMENT OF THE REQUIREMENTS \\ FOR THE DEGREE OF \\ DOCTOR OF PHILOSOPHY
}

Tetiana B. Hryn'ova

March 2006 
(C) Copyright by Tetiana B. Hryn'ova 2006

All Rights Reserved 
I certify that I have read this dissertation and that, in my opinion, it is fully adequate in scope and quality as a dissertation for the degree of Doctor of Philosophy.

Rafe H. Schindler Principal Co-Advisor

I certify that I have read this dissertation and that, in my opinion, it is fully adequate in scope and quality as a dissertation for the degree of Doctor of Philosophy.

\section{Peter C. Kim Principal Co-Advisor}

I certify that I have read this dissertation and that, in my opinion, it is fully adequate in scope and quality as a dissertation for the degree of Doctor of Philosophy.

\section{Stanley J. Brodsky}

I certify that I have read this dissertation and that, in my opinion, it is fully adequate in scope and quality as a dissertation for the degree of Doctor of Philosophy.

Patricia Burchat 
I certify that I have read this dissertation and that, in my opinion, it is fully adequate in scope and quality as a dissertation for the degree of Doctor of Philosophy.

\author{
Charles Prescott
}

Approved for the University Committee on Graduate Studies. 


\section{Acknowledgments}

There is no way I could possibly thank all those who have contributed to my graduate experience. I wish only to highlight those to whom I owe a particular debt of gratitude. Foremost, I would like to thank my advisors, Rafe Schindler and Peter Kim. Rafe, for supporting me all these years and allowing me to work on so many interesting projects, and Peter, without whom this work would not have been possible, for his contagious enthusiasm, unfaltering attention, and guidance both as a mentor and a friend. I would also particularly like to thank Bill Wisniewski, for his support and sage advice, Bob Cahn for many useful discussions and constant encouragement, and Bill Dunwoodie for reminding me that physics is more than just data analysis.

I would like to thank the members of Group E, for their support and help throughout my endeavors: Judy Meo and Melodi Masaniai, for creating a homey group environment so far away from home, Martin Perl, for being a wonderful group co-leader, Steven Robertson, for getting me started, Martin Kocian, Hojeong Kim, Johannes Bauer, Howard Rogers, Eric Lee and Valerie Halyo for being there for me. I would like to thank the members of my dissertation committee, Stanley Brodsky, Patricia Burchat, and Charles Prescott, for their time and many useful discussions throughout my graduate career. Many thanks to Jerome Friedman, for agreeing to chair the committee. I also thank Jim Allen, Ron Seefred, Justino Escalera, Mark Freitag, Lupe Salgado and Santa Chatterji for their help with my hardware project.

Finally, I would like to thank my best friends, Jörg Stelzer and Erkcan Özcan, for providing their companionship and advice, without them the past four years in Stanford would have been much less enjoyable experience. Огромное спасибо to my family, for their patience, support and understanding. But most of all I am indebted to my husband, Nicolas, for his support and encouragement through all these years. 


\section{Abstract}

$B$ mesons are unique among well-established non-quarkonium mesons in their ability to decay into baryons. Baryonic $B$ decays offer a wide range of interesting areas of study: they can be used to test our theoretical understanding of rare decay processes involving baryons, search for direct CP violation and study low-energy QCD.

This thesis presents measurements of branching fractions and a study of the decay dynamics of the charmless three-body decays of $B$ meson into $p \bar{p} h$ final states, where $h=\pi^{+}, K^{+}, K_{S}^{0}, K^{* 0}$ or $K^{*+}$. With a sample of 232 million $\Upsilon(4 S) \rightarrow B \bar{B}$ events collected with the BaBar detector, we report the first observation of the $B \rightarrow p \bar{p} K^{* 0}$ decay, and provide improved measurements of branching fractions of the other modes.

The distribution of the three final-state particles is of particular interest since it provides dynamical information on the possible presence of exotic intermediate states such as the hypothetical pentaquark states $\Theta^{*++}$ and $\Theta^{+}$in the $m_{p K^{+}}$and $m_{p K_{S}^{0}}$ spectra, respectively, or glueball states (such as the tensor glueball $f_{J}(2220)$ ) in the $m_{p \bar{p}}$ spectrum. No evidence for exotic states is found and upper limits on the branching fractions are set.

An enhancement at low $p \bar{p}$ mass is observed in all the $B \rightarrow p \bar{p} h$ modes, and its shape is compared between the decay modes and with the shape of the time-like proton form factor. A Dalitz plot asymmetry in $B \rightarrow p \bar{p} K^{+}$mode suggests dominance of the penguin amplitude in this decay and disfavors the possibility that the low mass $p \bar{p}$ enhancement originates from the presence of a resonance below threshold (such as the recently seen baryonium candidate at $\left.1835 \mathrm{MeV} / c^{2}\right)$.

We also identify decays of the type $B \rightarrow X_{c \bar{c}} h \rightarrow p \bar{p} h$, where $h=K^{+}, K_{S}^{0}, K^{* 0}$ or $K^{*+}$, and $X_{c \bar{c}}=\eta_{c}$ or $J / \psi$. In particular, we report on the evidence of the $B \rightarrow \eta_{c} K^{*+}$ decay and provide a measurement of the width of $\eta_{c}$. 


\section{Contents}

Acknowledgments $\quad$ v

Abstract $\quad$ vi

1 Introduction and Motivation 1

1.1 QCD and Exotics . . . . . . . . . . . . . . 1

1.2 Glueballs . . . . . . . . . . . . . . . . . . . . 3

1.3 Baryonium . . . . . . . . . . . . . . . . . . 6

1.4 New charmonium resonances . . . . . . . . . . . . . . . . . . 8

1.5 Pentaquarks . . . . . . . . . . . . . . . . . . . . 12

$1.6 \quad B$ decays . . . . . . . . . . . . . . . . . . . . 16

2 The BABAR Detector and Data 23

2.1 The PEP-II Collider . . . . . . . . . . . . . . . . . 23

2.2 The BABAR Detector . . . . . . . . . . . . . . . . . . 24

2.3 The Silicon Vertex Tracker . . . . . . . . . . . . . . 26

2.3.1 Layout and Electronics . . . . . . . . . . . . . . 26

2.3.2 Reconstruction and Performance . . . . . . . . . . . . . 28

2.4 The Drift Chamber . . . . . . . . . . . . . . . . . . . . . 29

2.4.1 Design and Geometry . . . . . . . . . . . . . . . . . . 29

2.4.2 Electronics and Readout . . . . . . . . . . . . . . 30

2.4.3 Calibration and Single-Cell Performance . . . . . . . . . . . . 32

2.4.4 Tracking and Performance . . . . . . . . . . . . . . 33

2.5 The Ring-Imaging Cerenkov Detector (DIRC) . . . . . . . . . . . 34 
2.5.1 Design and Geometry . . . . . . . . . . . . 35

2.5.2 Electronics and Reconstruction ........... . . 35

2.6 Trigger . . . . . . . . . . . . . . . . . . . . 38

2.6.1 The Level 1 Trigger . . . . . . . . . . . . . . . . . . 38

2.6.2 The Level 3 Trigger . . . . . . . . . . . . . . . . . 39

2.7 The Data . . . . . . . . . . . . . . . . . . . 40

$3 \quad B^{+} \rightarrow p \bar{p} K^{+}$Branching Fraction Measurement $\quad 42$

3.1 Track reconstruction requirements . . . . . . . . . . . . . . . . . 42

3.2 Particle Identification (PID) . . . . . . . . . . . . . . . . 43

$3.3 \quad B^{+} \rightarrow p \bar{p} K^{+}$Selection . . . . . . . . . . . . . . . . . 44

3.4 Background Characterization . . . . . . . . . . . . . 46

3.4.1 Continuum $(q \bar{q})$ Background . . . . . . . . . . . . 46

3.4.2 B Background . . . . . . . . . . . . . . . . . 49

3.5 Event Selection . . . . . . . . . . . . . . . . 50

3.6 Branching fraction measurement . . . . . . . . . . . . 50

3.6.1 $\mathcal{B}\left(B^{+} \rightarrow p \bar{p} K^{+}, m_{p \bar{p}}<2.85 \mathrm{GeV} / c^{2}\right)$ measurement . . . . . 53

3.6.2 $\mathcal{B}\left(B^{+} \rightarrow \eta_{c} K^{+}\right) \times \mathcal{B}\left(\eta_{c} \rightarrow p \bar{p}\right)$ and $\Gamma\left(\eta_{c}\right)$ measurements . . . 55

3.6.3 Total $\mathcal{B}\left(B^{+} \rightarrow p \bar{p} K^{+}\right)$measurement . . . . . . . . . . . . . . 57

3.6.4 Measurement of the charge asymmetry in $B^{+} \rightarrow p \bar{p} K^{+} \ldots$. . 60

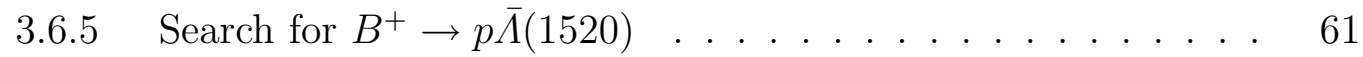

3.6.6 $\Theta^{*++}$ pentaquark search . . . . . . . . . . . . . 62

3.7 Systematic Studies . . . . . . . . . . . . . . . . 66

3.8 Summary . . . . . . . . . . . . . . . . . . . . . . 68

4 The $B \rightarrow p \bar{p} h$ Maximum Likelihood Analysis $\quad 69$

4.1 Event Selection . . . . . . . . . . . . . . . . 69

4.1.1 Track reconstruction . . . . . . . . . . . . . . 69

4.1 .2 Particle Identification . . . . . . . . . . . . . . . . 70

$4.1 .3 K_{S}^{0}$ Selection . . . . . . . . . . . . . . . 70

4.1.4 $K^{*+}$ Selection . . . . . . . . . . . . . . 72

4.1.5 $K^{* 0}$ Selection . . . . . . . . . . . . . 72 
$4.1 .6 \quad$ B Selection . . . . . . . . . . . . . . . . . . . . 72

4.1.7 Background Characterization . . . . . . . . . . . 72

4.1.8 Summary of the event selection. . . . . . . . . . . . . 73

4.2 Maximum Likelihood Fit . . . . . . . . . . . . . . . . 76

4.2.1 Probability Distribution Function (PDF) Parameterization . . 80

4.2 .2 Signal Efficiency _ . . . . . . . . . . . . . . . 85

4.3 Systematic Errors . . . . . . . . . . . . . . . . . . . . 85

4.3.1 Fit Validation with Monte Carlo Samples . . . . . . . . . . 86

4.3.2 Efficiency Corrections and Systematic Errors . . . . . . . . . 87

4.3 .3 Monte Carlo statistics . . . . . . . . . . . . . . . . . . 89

4.3.4 Pre-selection cuts . . . . . . . . . . . . . . . . . . . . 89

4.3 .5 Fit Region . . . . . . . . . . . . . . . . . . . . . 89

4.3.6 Summary of Systematic Uncertainties . . . . . . . . . . . . 89

4.4 Summary of Results _ . . . . . . . . . . . . . . . . . . 92

4.4.1 $B \rightarrow p \bar{p} h$ Branching Fraction Measurements . . . . . . . . . 92

4.4.2 Study of Charmonium Substructure _ . . . . . . . . . . 97

4.4.3 $B \rightarrow \Lambda_{c}^{+} \bar{p}$ Branching Fraction Measurement . . . . . . . . 99

4.4.4 $\mathcal{B}\left(B^{0} \rightarrow \Theta(1540)^{+} \bar{p}\right)$ Upper Limit Calculation . . . . . . . . 102

4.4.5 Search for glueballs in $B \rightarrow p \bar{p} h$ decays . . . . . . . . . . 104

4.5 Summary . . . . . . . . . . . . . . . . . . . 105

5 Summary and Outlook 106

5.1 Summary of Branching Fraction Values . . . . . . . . . . . . 106

5.2 Study of the $B \rightarrow p \bar{p} h$ decay dynamics _ . . . . . . . . . 107

5.2 .1 Low $m_{p \bar{p}}$ enhancement . . . . . . . . . . . . . . 109

$5.2 .2 \quad$ Dalitz plot asymmetry . . . . . . . . . . . . . . . . . . 109

5.3 Exotic searches . . . . . . . . . . . . . . . . . . . . . . . . 111

5.4 Future Prospects . . . . . . . . . . . . . . . . . . . . . . 111

A Three-body decay kinematics $\quad 114$

A.1 Phase-space . . . . . . . . . . . . . . . . . . . . . . . . 114

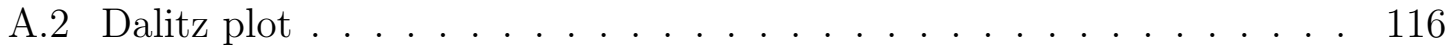


B Study of Radiation Damage of CsI(Tl) Crystals 118

B.1 The Electromagnetic Calorimeter . . . . . . . . . . . . . . . 118

B.1.1 Geometry and Electronics . . . . . . . . . . . . . 118

B.1.2 Reconstruction and Performance . . . . . . . . . . . 119

B.2 Radiation Damage . . . . . . . . . . . . . . . . . . . 121

B.2.1 Effects of Radiation on Crystals . . . . . . . . . . . . 121

B.2.2 Sources of Radiation Damage . . . . . . . . . . . . . 121

B.3 Dose Monitoring . . . . . . . . . . . . . . . . . . . . . 122

B.3.1 RadFET Monitoring . . . . . . . . . . . . . . . . 122

B.3.2 Leakage Currents . . . . . . . . . . . . . . . . . . . . 122

B.4 Total Light Yield (LY) Monitoring . . . . . . . . . . . . . . 123

B.4.1 Source Measurements . . . . . . . . . . . . . . . . . 125

B.4.2 Bhabha Measurements . . . . . . . . . . . . . 126

B.5 Study of Light-Yield Uniformity along the Crystal Length _. . . . 127

B.5.1 Experimental Setup . . . . . . . . . . . . . . . 128

B.5.2 Measurements . . . . . . . . . . . . . . . . . . . . 129

B.5.3 Study of the impact of non-uniformity . . . . . . . . . . 133

B.5.4 Conclusion . . . . . . . . . . . . . . . . . . 135

$\begin{array}{ll}\text { Bibliography } & 137\end{array}$ 


\section{List of Tables}

1.1 Possible spin-parity combinations for $g g$ glueball states from Ref. [13]. 3

1.2 Predicted and observed masses of $c \bar{c}$ states (in MeV) from Ref. [53] . 9

1.3 Experimental results with positive signals for $\Theta^{+}$state. . . . . . . 13

1.4 Predictions of the branching ratios for some two-body baryonic $B$ decays. 17

1.5 Summary of the experimental and theoretical values for the branching fractions $\left(\times 10^{-6}\right)$ of $B \rightarrow p \bar{p} h$. . . . . . . . . . . . . . 20

2.1 Generic Monte Carlo samples used in the analysis. . . . . . . . . . . . 40

3.1 The expected rates for the events of the type $B^{+} \rightarrow X K^{+}, X \rightarrow p \bar{p}[20] .50$

3.2 $\Delta E$ fit results used for the $B^{+} \rightarrow p \bar{p} K^{+}$branching fraction calculation. 55

3.3 Summary of the $B^{+} \rightarrow p \bar{p} K^{+}$asymmetry study. . . . . . . . . . . . . 61

3.4 Upper limits for the branching fraction of $B^{+} \rightarrow \Theta^{*++}\left(p K^{+}\right) \bar{p}$ as a function of $m_{p K^{+}}$without (with) background subtraction. . . . . . . 67

3.5 Systematic uncertainties in percent on the branching fraction measurements and in the values of uncertainties for the asymmetry measurements. 67

4.1 The expected rates for the peaking background events of the type $B^{0} \rightarrow$ $X h, X \rightarrow p \bar{p}$ and $h=K^{0}, K^{*+}, K^{* 0}, \pi^{+} \ldots \ldots \ldots 74$

4.2 Estimated non-charmonium $B$ background events with $p \bar{p} h$ signal final states in $210 \mathrm{fb}^{-1}$ of data. . . . . . . . . . . . . . . . . 75

4.3 Summary of the event selections. . . . . . . . . . . . . 75

4.4 Maximum Likelihood Fit Event Categories. . . . . . . . . . . . . . . 76 
4.5 The total number of event categories $\left(N_{s}\right)$ in Maximum Likelihood Fit Regions. . . . . . . . . . . . . . . . 78

4.6 Maximum Likelihood Fit Variables. . . . . . . . . . . . . . . . 78

4.7 The PDF parametrization of $B \rightarrow p \bar{p} h$ signal and background. . . . . 81

4.8 Systematic errors (in percent) and vertexing efficiency corrections for all modes. . . . . . . . . . . . . . . . . . . . . . 90 90

4.9 Systematic errors (in percent) and efficiency corrections (cor) for the charmonium decays. . . . . . . . . . . . . . . . . . . . . 90

4.10 Systematic errors (in percent) and efficiency corrections for the $\Lambda_{c}^{+}$ decays. . . . . . . . . . . . . . . . . . . . 91

4.11 Systematic errors (in percent) and efficiency corrections for the exotic searches. . . . . . . . . . . . . . . . . . 91

4.12 Summary of the resulting yields from the ML fit for all modes. . . . . 93

4.13 Non-resonant $B \rightarrow p \bar{p} h$ branching fractions after $B$ background and $K^{*}$ sideband subtractions where applicable. . . . . . . . . . . . 96

4.14 Comparison of non-resonant $B \rightarrow p \bar{p} h$ branching fractions from Ref. [131, 132] and this work. . . . . . . . . . . . . . . 96

4.15 Summary of the asymmetry study. Resulting yields from the ML fit for all modes. . . . . . . . . . . . . . . . . . . . . . . 100

4.16 Summary of the resulting branching fractions for $\eta_{c}$ and $J / \psi$ modes. . 100

5.1 Summary of the experimental values for the branching fractions $\left(\times 10^{-6}\right)$ of $B \rightarrow p \bar{p} h$ and their comparison to two-body mesonic modes from Ref. [149]. . . . . . . . . . . . . . . . . 106

B.1 Parametrization of $\Delta(D)$ in percent . . . . . . . . . . . . . . 133

B.2 Estimate of the EMC resolution for single photons in \% . . . . . . 135 


\section{List of Figures}

1.1 Predicted glueball spectrum from the Lattice QCD model from Ref. [15]. 5

1.2 The current state of knowledge of the charmonium system and transitions from Ref. [20]. . . . . . . . . . . . . . . . . . . 10

1.3 The predicted anti-decuplet [86] of pentaquark baryons. . . . . . . . . 12

1.4 Idealization [129] of the main Feynman diagrams for the non-resonant $B^{+} \rightarrow p \bar{p} K^{+}$decay. . . . . . . . . . . . . . . . . . . 18

1.5 (a) The current-produced $(\mathcal{J})$ and (b) transition $(T)$ diagrams for $B^{+} \rightarrow p \bar{p} K^{+}$decay from Ref. [28]. . . . . . . . . . . . . . . . . 19

2.1 Longitudinal cross-section of the BABAR detector. . . . . . . . . . 25

2.2 Longitudinal cross-section of the SVT. . . . . . . . . . . . . 27

2.3 Longitudinal cross-section of the DCH. . . . . . . . . . . . . . . 29

2.4 Schematic layout of the drift cells in the four inner DCH superlayers. 31

2.5 DCH single cell resolution. . . . . . . . . . . . . . . . . . . 32

2.6 (a) Elevated view of overall DIRC geometry. (b) Bar/SOB transition region. . . . . . . . . . . . . . . 36

3.1 (a) $\Delta E$ distribution and (b) $m_{E S}$ distribution of truth-matched $B^{+} \rightarrow$ $p \bar{p} K^{+}$signal Monte Carlo events. . . . . . . . . . . . . . . 45

3.2 (a) $\Delta E$ distribution and (b) $m_{E S}$ distribution of off-peak data background events reconstructed in $B^{+} \rightarrow p \bar{p} K^{+}$mode. . . . . . . . . . . 45

$3.3 B^{+} \rightarrow p \bar{p} K^{+}$signal Monte Carlo (histogram) - off-peak data background (dots) comparisons for: (a) $L_{2}$, (b) $L_{0}$, (c) $\cos \theta_{t h r}^{B}$, and (d) $\cos \theta_{\text {mom }}^{B} \ldots \ldots \ldots \ldots \ldots \ldots$. . . . . . . . . . . . . . . . . 47 
3.4 (a) Fisher discriminant in signal Monte Carlo (histogram) and off-peak data (dots); (b) Fisher discriminant cut optimization. . . . . . . . . . 49

3.5 Distribution of $\Delta E$ versus $m_{E S}$ for the $p \bar{p} K^{+}$candidates in on-resonance data. . . . . . . . . . . . . . . . . 51

3.6 (a) $\Delta E$ distribution of on-peak data $\left(5.27<m_{E S}<5.29 \mathrm{GeV} / c^{2}\right)$; (b) $m_{E S}$ distribution of on-peak data $(|\Delta E|<0.05 \mathrm{GeV})$. . . . . . . 51

3.7 (a) $\Delta E$ distribution of on-peak data $\left(5.27<m_{E S}<5.29 \mathrm{GeV} / \mathrm{c}^{2}\right)$; (b) $m_{E S}$ distribution of on-peak data $(|\Delta E|<0.05 \mathrm{GeV})$. . . . . . . . 52

3.8 Reconstructed efficiency of $B^{+} \rightarrow p \bar{p} K^{+}$signal events as a function of

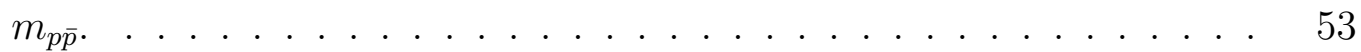

$3.9 \Delta E$ distribution for on-peak data in different $m_{p \bar{p}}$ regions for $B^{+} \rightarrow$ $p \bar{p} K^{+} \ldots \ldots \ldots \ldots \ldots 4$

3.10 The $m_{p \bar{p}}$ distribution for data in the wide signal (points) and sideband (shaded) regions. . . . . . . . . . . . . . . 56

3.11 Efficiency-corrected yield of $B^{+} \rightarrow p \bar{p} K^{+}$events as a function of $m_{p \bar{p}}$. 58

3.12 (a) The near threshold enhancement observed in $M_{p \bar{p}}-2 m_{p}$ distribution for the $J / \psi \rightarrow \gamma p \bar{p}$ event sample from Ref. [34]. (b) The same $M_{p \bar{p}}-$ $2 m_{p}$ distribution with events weighted by $q_{0} / q$. (c) Near threshold distribution of $m_{p \bar{p}}$ for the $p \bar{p} K^{+}$event sample. (d) The same $m_{p \bar{p}}$ distribution in the $B^{+} \rightarrow p \bar{p} K^{+}$sample with events weighted by $q_{0} / q$.

3.13 Phase-space-corrected $m_{p \bar{p}}$ distribution of $B^{+} \rightarrow p \bar{p} K^{+}$signal events (blue dots) compared to the effective form factor from $e^{+} e^{-} \gamma \rightarrow \gamma p \bar{p}$ [145] (red squares). . . . . . . . . . . . . . . . . 60

3.14 The $m_{\bar{p} K^{+}}$distribution for data events (points) in $B^{+} \rightarrow p \bar{p} K^{+}$signal box and $\Lambda(1520)$ mass region. . . . . . . . . . . . . . . .

3.15 (a) The $m_{\bar{p} K^{+}}$distribution for data events in $B^{+} \rightarrow p \bar{p} K^{+}$signal box and $\Lambda(1520)$ mass region. Results of the maximum likelihood fit are overlaid. (b) Likelihood distribution as a function of the branching fraction of $B^{+} \rightarrow p \bar{\Lambda}(1520)$. . . . . . . . . . . . . . . 63

3.16 The $B^{+} \rightarrow p \bar{p} K^{+}$signal reconstruction efficiency (a) and the detector resolution $(\mathrm{b})$ as functions of $m_{p K^{+}} \ldots \ldots \ldots$. . . . . . . 
3.17 The $m_{p K^{+}}$distribution for data events in $B^{+} \rightarrow p \bar{p} K^{+}$signal box. . .

3.18 The $m_{p K^{+}}$distributions for data reconstructed as $B^{+} \rightarrow p \bar{p} K^{+}$(solid line) and rescaled $m_{E S}$ sideband (dots). . . . . . . . . . . . . .

3.19 Upper Limit on the branching fraction of $B^{+} \rightarrow \Theta^{*++}\left(p K^{+}\right) \bar{p}$ at $90 \%$ confidence level. . . . . . . . . . . . . . . . . . . .

4.1 Relevant distributions of discriminating variables: (a) $\cos \alpha_{K_{S}^{0}}$, (b) $K_{S}^{0}$ vertex probability, (c) $K_{S}^{0}$ mass and (d) $B$ vertex probability. . . . . . 71

$4.2 \quad B^{0} \rightarrow p \bar{p} K_{S}^{0}$ Dalitz plot with bands of sub-resonance structures. . . . 77

4.3 PDFs for background in $B^{+} \rightarrow p \bar{p} K^{*+}$ mode: (a) $\Delta E$, (b) $m_{\mathrm{ES}}$, (c) Fisher and $(\mathrm{d}) m_{p \bar{p}} \ldots \ldots \ldots$. . . . . . . . . . . . . . . . . . 82

4.4 PDFs for signal events in $B^{+} \rightarrow p \bar{p} K^{*+}$ mode. . . . . . . . . . . . . . 83

4.5 PDFs for peaking $B$ background in $B^{0} \rightarrow p \bar{p} K^{0}$ mode. . . . . . . . . 84

4.6 (a) Signal efficiency; (b) absolute error on the signal efficiency; (c) relative error on the signal efficiency as a function of the Dalitz plot in $B^{0} \rightarrow p \bar{p} K^{0}$ mode. . . . . . . . . . . . . . . .

4.7 Distributions of $\Delta E, m_{\mathrm{ES}}$, and $\mathcal{F}$ for signal $B^{0} \rightarrow p \bar{p} K^{0}$ (a)-(c), $B^{+} \rightarrow$ $p \bar{p} K^{*+}(\mathrm{d})-(\mathrm{f}), B^{0} \rightarrow p \bar{p} K^{* 0}(\mathrm{~g})-(\mathrm{i}), B^{+} \rightarrow p \bar{p} \pi^{+}(\mathrm{j})$-(l) decays using the weighting technique described in Ref. [150]. . . . . . . . . .

4.8 Distributions of $\Delta E, m_{\mathrm{ES}}$, and $\mathcal{F}$ for combinatorial $B^{0} \rightarrow p \bar{p} K^{0}$ (a)$(\mathrm{c}), B^{+} \rightarrow p \bar{p} K^{*+}(\mathrm{d})-(\mathrm{f}), B^{0} \rightarrow p \bar{p} K^{* 0}(\mathrm{~g})-(\mathrm{i}), B^{+} \rightarrow p \bar{p} \pi^{+}(\mathrm{j})-(\mathrm{l})$ decays using the weighting technique described in Ref. [150]. . . . . . 95

4.9 The results of the ML fit for $m_{\mathrm{ES}}>5.27 \mathrm{GeV} / c^{2}$ for $B^{0} \rightarrow p \bar{p} K^{0}$ (a)-(c), $B^{+} \rightarrow p \bar{p} K^{*+}(\mathrm{d})-(\mathrm{f})$ and $B^{0} \rightarrow p \bar{p} K^{* 0}(\mathrm{~g})$-(i) modes. . . . . . . . . 98

4.10 The results of the ML fit in the $\Lambda_{c}^{+}$region for $m_{\mathrm{ES}}>5.27 \mathrm{GeV} / c^{2}$ for $B^{0} \rightarrow p \bar{p} K^{0}(\mathrm{a})-(\mathrm{c})$ and $B^{0} \rightarrow p \bar{p} K^{* 0}(\mathrm{~d})$-(f) modes. . . . . . . . . . . 101

4.11 sPlot of the $p K_{S}^{0}$ mass projections in $\Theta^{+}$mass region for $B^{0} \rightarrow p \bar{p} K^{0}$ signal events. . . . . . . . . . . . . . . . . . 103

4.12 Upper limits at $90 \% \mathrm{CL}$ on the product of branching fractions $\mathcal{B}(B \rightarrow$ $\left.f_{J}(2220) X\right) \times \mathcal{B}\left(f_{J}(2220) \rightarrow p \bar{p}\right)$ for $(\mathrm{a}) B^{+} \rightarrow p \bar{p} K^{+},(\mathrm{b}) B^{0} \rightarrow p \bar{p} K^{0}$, (c) $B^{+} \rightarrow p \bar{p} K^{*+}$ and (d) $B^{0} \rightarrow p \bar{p} K^{* 0}$ modes. . . . . . . . . . . 104 
5.1 Dalitz sPlots for $B \rightarrow p \bar{p} h$ modes. . . . . . . . . . . . . . 108

$5.2 m_{p \bar{p}}$ distribution sPlots for $B \rightarrow p \bar{p} h$ modes. . . . . . . . . . 110

5.3 Comparisons between sPlots of $m_{p \bar{p}}$ distributions for (a) $B^{0} \rightarrow p \bar{p} K^{0}$ (errors) vs $B^{0} \rightarrow p \bar{p} K^{* 0}$ (histogram), (b) $B^{+} \rightarrow p \bar{p} K^{+}$(errors) vs $B^{+} \rightarrow p \bar{p} K^{*+}$ (histogram) and (c) rescaled to the same area $B^{+} \rightarrow$ $p \bar{p} K^{+}$(histogram) vs $B^{0} \rightarrow p \bar{p} K^{0}$ (errors). . . . . . . . . .

$5.4 m_{p h}$ distribution sPlots in red for $m_{p h}>m_{\bar{p} h}$ and in black for $m_{p h}<$ $m_{\bar{p} h}$ for (a) $B^{0} \rightarrow p \bar{p} K^{0}$, (b) $B^{+} \rightarrow p \bar{p} K^{+}$, (c) $B^{0} \rightarrow p \bar{p} K^{* 0}$, (d) $B^{+} \rightarrow p \bar{p} K^{*+}$ and (e) $B^{+} \rightarrow p \bar{p} \pi^{+}$modes. . . . . . . . . 112

B.1 (a) Longitudinal section of EMC. (b) Crystal housing with front-end electronics . . . . . . . . . . . . . . . . . . . . . . . . . . . 119

B.2 Average dose in the EMC measured by (left) the RadFETs, (right) the leakage currents. . . . . . . . . . . . . . . .

B.3 Average dose in the EMC measured by the leakage currents depending on the beam condition. . . . . . . . . . . . . . .

B.4 Average change in the light yield in the EMC measured with (a) source (August 1999-December 2001), (b) source(s) and Bhabhas(B) (January-December 2001) . . . . . . . . . . . . . . . . . 125

B.5 Average change in the LY in the EMC by vendor. . . . . . . . 126

B.6 Experimental Setup: crystal array on the left, ${ }^{60} \mathrm{Co}$ source on the right. 128

B.7 a. Typical uniformity scan results for Type A crystal; b. Irradiation contribution to the uniformity. . . . . . . . . . . . . 130

B.8 a. Typical uniformity scan results for Type B crystal; b. Irradiation contribution to the uniformity. . . . . . . . . . . . . .

B.9 Dose dependence of the percentage drop of the light yield for a sample of different crystal types. . . . . . . . . . . . . . . .

B.10 MC study of the non-uniformity contribution to the energy resolution $\left(C_{u}\right)$ dependence on the total drop of the light yield $\left(\Delta_{t o t}\right)$. The error bars show typical uncertainties in the curves . . . . . . . . . 134 


\section{Chapter 1}

\section{Introduction and Motivation}

Three-body baryonic $B$ decays offer a clean environment to search for intermediate states, both exotic states (such as glueballs, in particular tensor glueball $f_{J}(2220)$, pentaquarks $\Theta^{+}(1540)$ and $\Theta^{*++}$, and baryonium) and non-exotic states (new charmonium resonances), as well as to study the properties of the known charmonium states. These decays can also be used to test our theoretical understanding of rare decay processes involving baryons, to search for direct $\mathrm{CP}$ violation, and to investigate low-energy QCD.

\subsection{QCD and Exotics}

In 1968, deep inelastic electron scattering experiments at SLAC, Stanford, California, gave the first clear evidence that point-like particles, so-called "partons", exist inside the proton [1]. These "partons" were later identified with the "quarks" predicted by Gell-Mann and Zweig [2]. All the known baryon and meson resonances at the time could be built out of a unitary triplet consisting of a quark doublet $(u, d)$ and a singlet $(s)$ with charge $\left(\frac{2}{3} e,-\frac{1}{3} e,-\frac{1}{3} e\right)$, strangeness $(0,0,-1)$ and baryon number $\left(\frac{1}{3}, \frac{1}{3}, \frac{1}{3}\right)$, respectively. In 1964 discovery of the $\Omega$ particle [3] with strangeness -3 led to introduction of a new type of charge called "color" [4]. The 1974 discovery of the charm quark at SLAC and BNL [5] lead to the establishment of a theory of strong interactions, "quantum chromodynamics"(QCD) [6]. 
QCD is a quantum field theory for the strong interactions, a non-abelian gauge field theory based on the gauge group $\mathrm{SU}(3)_{c}$ for quarks with massless vector particles mediating the force. In QCD the strong interaction between colored quarks occurs via the exchange of virtual particles, gluons, similar to the interaction between electrically charged particles mediated by the exchange of virtual photons. While photons electrically are neutral and therefore do not interact with each other, gluons carry color "charges" and are capable of interacting between themselves. Failure to observe free quarks experimentally [7] led to the concept of "confinement", according to which only the particles that are singlets of $\mathrm{SU}(3)_{c}$ can exist. Hadrons are then interpreted as bound states of quarks held together by gluons. Furthermore, all known hadrons can be described as color-antisymmetric combinations of either $q \bar{q}$, called mesons, or $q q q$, called baryons. These valence structures are surrounded by a virtual "sea" of quark-antiquark pairs and gluons, but the fundamental valence quarks determine the quantum numbers of the hadron. QCD has been tested and verified extensively by many experiments, and observations.

Nothing however, explicitly forbids the existence of other color-singlet combinations, such as multiquark mesons $(q q \bar{q} \bar{q})$ [8], pentaquark baryons $(q q q q \bar{q})[9]$, and baryonium $(q q q \bar{q} \bar{q} \bar{q})[10]$. The advent of QCD led to the natural assumption that gluons could also be part of the fundamental valence structural elements. For example "glueball" states, consisting of gluons only $(g g(g \ldots))$ [11], or hybrids, consisting of valence quarks and gluons ( $q \bar{q} g$ or $q q q g)$ [12] might exist. The term "exotic" hadrons is used to refer to these particles.

There exist a considerable number of theoretical models of exotic hadrons. Exotic particles may even consist of bound states of non-exotic colorless hadrons, and can thus decay into their colorless components without the creation of additional $q \bar{q}$ pairs from the vacuum. In the absence of kinematic suppression, however, decays of this kind can have considerable width, making them very difficult to observe experimentally.

It has also been suggested that there are relatively narrow exotic states within the complex internal color structure of these objects and within the singularities of color dynamics. If an exotic hadron consists of two colored parts that are separated 
Table 1.1: Possible spin-parity combinations for $g g$ glueball states from Ref. [13].

\begin{tabular}{||c|c||}
\hline $\mathrm{L}$ & $J^{P C}$ \\
\hline 0 & $0^{++}, 2^{++}$ \\
\hline 1 & $0^{-+}, 1^{-+}, 2^{-+}$ \\
\hline 2 & $2^{++}, 0^{++}, 4^{++}$ \\
\hline 3 & $2^{-+}, 3^{-+}, 4^{-+}$ \\
\hline
\end{tabular}

in space (e.g., because of the presence of a centrifugal barrier), then its decay to the color-singlet final states will be suppressed. Such exotic particles can be characterized by narrow decay widths, and hence are more accessible to experimental searches.

\section{$1.2 \quad$ Glueballs}

In QCD, gluons carry color charge and consequently they can interact with other gluons leading to couplings between two and three gluons. Hence, it may be possible for gluons to form bound states entirely composed of gluons with no quarks. These bound states of gluons are called "glueballs".

Gluons are spin-one vector particles and members of a color octet. The possible spin-parity combinations for two-gluon glueball states $(g g)$ are summarized in Table 1.1. Several modes in the table are impossible to form from $q \bar{q}$ meson states. For example, for neutral $(q \bar{q})$ mesons with total quark spin $S$ and orbital angular momentum $L$, the parity and charge conjugation quantum numbers are known to be given by $P=-(-1)^{L}, C=(-1)^{L+S}$, so that such mesons can only have the following combinations of quantum numbers $\left(J^{P C}\right)$ :

- for $L=0$ only $0^{-+}$and $1^{--}$states are possible,

- for $L=o d d$ : odd $d^{++}$, even $^{++}, o d d^{+-}$,

- for $L=\operatorname{even}(>0)$ : even ${ }^{-+}$, odd ${ }^{--}, e^{e v e n^{--}}$.

The exotic sets of quantum numbers not accessible to mesons are thus $J^{P C}=$ $0^{+-}, 0^{--}, 1^{-+}, 2^{+-}, 3^{-+}$, etc. 
From Table 1.1 we see that in the case of $g g$ glueballs, the only exotic combinations accessible $^{1}$ are the $J^{P C}=1^{-+}, 3^{-+}$, while they are all possible for $g g g$ glueballs.

Figure 1.1 shows predicted glueball spectrum from the Lattice QCD calculations [15]. The shown values of glueball masses where obtained by taking the lattice spacing to zero and scale parameter $r_{0}$ to be $\sim 0.5 \mathrm{fm}$ (as estimated from the string tension in heavy quark mesons). From Figure 1.1, the lightest glueballs are expected to have quantum numbers $J^{P C}=0^{++}$and $2^{++}$and masses [16] of $1611 \pm 163 \mathrm{MeV} / c^{2}$ and $2232 \pm 310 \mathrm{MeV} / c^{2}$, respectively. The glueballs with the exotic quantum numbers are predicted to lie far above $2 \mathrm{GeV} / c^{2}$. The lightest glueball with exotic quantum numbers $\left(2^{+-}\right)$has a mass of about $4 \mathrm{GeV} / c^{2}$. This is a region of mesons with higher radial and orbital excitations, where the states become increasingly broad and overlap, making any search difficult.

The glueball width is not predicted by lattice QCD. There are very rough ideas that the width should be between the width of conventional mesons and the partial width of Okubo-Zweig-Iizuka (OZI) suppressed decays. The width of an OZIsuppressed $\phi$ decay is $\sim 0.5 \mathrm{MeV}$; the width of a typical not-OZI-suppressed meson decay of the $\rho$ meson is $150 \mathrm{MeV}$ :

$$
\Gamma_{g g}=\sqrt{\Gamma_{O Z I} \Gamma_{q \bar{q}}} \sim \text { few 10s of MeV. }
$$

The expected features of a glueball are: (i) no place in $q \bar{q}$ nonets, (ii) enhanced production in gluon-rich channels, such as radiative $J / \psi$ decays and OZI-suppressed decay channels, (iii) decay rates incompatible with those of $q \bar{q}$ states and (iv) no radiative decays and no two-photon decays. As the lowest glueball states lie in a region rich in $q \bar{q}$ resonances of the same $J^{P C}$, mixing also becomes possible. This perturbs the mass spectrum, complicating the isolation of a glueball signal and leads to too many observed states with the same quantum numbers. Hence a careful understanding of conventional $q \bar{q}$ meson spectroscopy is necessary to interpret any glueball candidate.

The treatment of mesons as $q \bar{q}$ systems bound in a phenomenological QCD confining potential [17] provides a reasonable description of the whole spectrum of known

\footnotetext{
${ }^{1}$ The existence of a $g g$ glueball with spin $J=1$ is doubtful, because of the Landau-Yang theorem that forbids such states for massless gluons [14]
} 


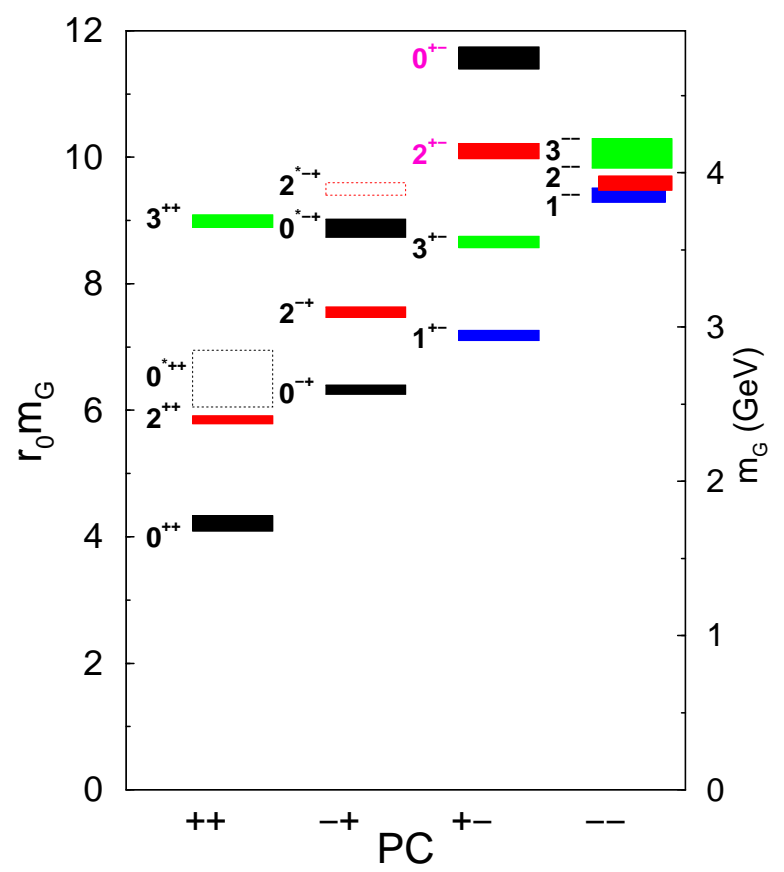

Figure 1.1: Predicted glueball spectrum from the Lattice QCD model from Ref. [15]. meson states, including those involving heavy quarks. In this model, the $a^{0}(980)$ and $f^{0}(980)$ scalar mesons are described as $K \bar{K}$ bound states with no isoscalar or strange meson partners [18]. An alternative bag-model description $[9,19]$ finds that they can occur in a low-mass nonet of "cryptoexotic" $(q q \bar{q} \bar{q})$ states, thus going beyond the $q \bar{q}$ description. However, this would imply the existence of strange and isoscalar members of such a nonet, the $\kappa$ and $\sigma$, respectively; there is an extensive debate as to whether these states exist [20].

One area of particular difficulty for the $q \bar{q}$ description involves the higher mass isoscalar mesons with $J^{P C}=0^{++}$: there are too many (three) such states in the region $1.35-1.75 \mathrm{GeV} / c^{2}: f_{0}(1370), f_{0}(1500)$ and $f_{0}(1700)$. Lattice calculations [15] predict that the lightest glueball lies between 1.4 and $1.8 \mathrm{GeV} / c^{2}$. In one of the interpretations $[11,20]$ the isoscalars $f_{0}(1370)$ and $f_{0}(1700)$ contain small fractions of glue, while $f_{0}(1500)$ is mostly gluonic.

There is some experimental evidence for a narrow structure $f_{J}(2220)$ reported around $2230 \mathrm{MeV} / \mathrm{c}^{2}$, where the $2^{++}$glueball state is expected. In 1986 the MARK III collaboration reported evidence for a narrow resonance at $\sim 2230 \mathrm{MeV} / c^{2}$ with a width 
of $\sim 20 \mathrm{MeV}$ in the invariant mass spectrum of $K^{+} K^{-}$and $K_{S} K_{S}$ [21] in radiative $J / \psi$ decays. In 1996 the BES Collaboration reported new evidence $(\sim 4 \sigma)$ for this state in their analysis of radiative $J / \psi$ decays [22]. Not only did they find a narrow signal in the $K^{+} K^{-}$and $K_{S} K_{S}$ spectra in agreement with the previous measurement, but they also claimed additional decay modes of $f_{J}(2220) \rightarrow \pi^{+} \pi^{-}, \pi^{0} \pi^{0}$ and $p \bar{p}$, all with product branching fractions $\mathcal{B}\left(J / \psi \rightarrow \gamma f_{J}(2220)\right) \times \mathcal{B}\left(f_{J}(2220) \rightarrow h h\right)$ in the range of $(1.5-5.6) \times 10^{-6}$. The nearly flavor-blind decay of the $f_{J}(2220)$, its production in the gluon rich environment of $J / \psi$ radiative decay, its non-observation in $\gamma \gamma$ collisions [23], its narrow width, $J^{P C}=(\text { even })^{++}$, and its mass in the range of lattice predictions, makes this state an attractive candidate for the $2^{++}$glueball.

However the DM2 collaboration repeated the measurement of MarkIII and found no indication of a narrow state [24], setting limits on the rates that are of the same order as the claimed observations. The Crystal Barrel collaboration performed a scan of the mass region $\sqrt{s}=2222.7-2239.7 \mathrm{MeV} / c^{2}$ investigating $\pi^{0} \pi^{0}$ and $\eta \eta$ final states: no indication of the formation of $f_{J}(2220)$ was found [25]. Combining the upper limit in $p \bar{p}$ formation and the claimed decay to $p \bar{p}$ gives an upper limit for the process $J / \psi \rightarrow \gamma f_{J}(2220)$ of $\sim 2.3 \times 10^{-3}$ [26], which should be visible in the inclusive photon spectrum [27]. There is also a recent speculation that it might be seen in charmless $B$ decay to $p \bar{p} K^{+}[28]$. This possibility is investigated in the current work.

\subsection{Baryonium}

Theoretical investigations of baryon-antibaryon bound states date back to the proposal of Fermi and Yang [29] to make the pion from a nucleon-antinucleon pair. The model of Nambu and Jona-Lasinio [30], which is constructed to give a nearly zeromass pion as a fermion-antifermion bound state, also has a scalar resonance of twice the fermion mass. The short range nucleon-nucleon interaction is repulsive, presumably due to heavy meson $t$-channel exchanges (e.g., $\omega$ exchange). Through $G$-parity transformation the interaction becomes attractive for various partial waves of the proton-antiproton system, and a rich spectrum of bound states and resonances for 
the proton-antiproton system is predicted [31].

In the 1970's there were many indications of new mesons coupled to the nucleonnucleon system. A comprehensive search for baryonium conducted at the Low Energy Antiproton Ring (LEAR) at CERN resulted in no clear evidence for the existence of baryonium [32]. With the possible exception of the $f_{2}(1565)$ candidate [33], none of the baryonium states were observed, perhaps because they easily decay into mesons and are therefore very broad. Also, the predictions for bound states rely on the short range attraction of the nucleon-nucleon interaction, which may instead be mediated by a one-gluon exchange spin-spin contact interaction, in which case $\bar{p} p$ and $p p$ are not related by $G$-parity transformation [25].

The recent rise of interest in baryonium is due to the observation of a nearthreshold enhancement in the $p \bar{p}$ invariant mass spectrum, in $J / \psi \rightarrow \gamma p \bar{p}$ decay [34]. No similar signal is observed in the channel $J / \psi \rightarrow \pi^{0} p \bar{p}$. Ignoring final state interactions, the central value of the mass of this enhancement from an S-wave fit was around $1859 \mathrm{MeV} / c^{2}$ [34]. With final state interactions in the isoscalar channel calculated in Ref. [35], BES refit the mass and found it to lie around $1830 \mathrm{MeV} / \mathrm{c}^{2}$ [36]. The proximity of this resonance to the $p \bar{p}$ threshold nourished speculations that the observed strong enhancement could be the signature of an $N \bar{N}$ bound state [37]. In order to establish this enhancement as a new resonance, it was suggested [38] that if this state is indeed baryonium it must be observed in mesonic decay channels such as $\eta \pi \pi, \eta^{\prime} \pi \pi$ and others. Subsequently, the BES Collaboration reported a $7.7 \sigma$ signal of $\mathrm{X}(1835)$ in $J / \psi \rightarrow \eta^{\prime} \pi \pi$ decays with mass of $1833.7 \pm 6.2 \pm 2.7 \mathrm{MeV} / c^{2}$ and width of $67.7 \pm 20.3 \pm 7.7 \mathrm{MeV}$ [39]. No signal was observed in the $\eta \pi \pi$ channel.

At about the same time evidence for low-mass $p \bar{p}$ enhancements with lower statistical significance were reported in $B^{+} \rightarrow p \bar{p} K^{+} \quad$ [40] and $\bar{B}^{0} \rightarrow D^{0} p \bar{p}$ decays [41], $\Lambda \bar{p}$ enhancements in $J / \psi \rightarrow \bar{p} K^{-} \Lambda$ [42] and $B^{+} \rightarrow \bar{p} \Lambda \pi(\gamma)$ decays [43], and a $\Lambda \bar{\Lambda}$ enhancement in $B \rightarrow \Lambda \bar{\Lambda} K^{+/ 0}$ decays [44].

There are alternative explanations for these enhancements as gluonic states [45, 46] or the result of the quark fragmentation process [46]. An entirely different and much more conventional interpretation of the observed enhancements is suggested in several recent works $[35,47,48,50]$. These authors argue that the enhancements are 
primarily due to final state interactions (FSI) between the produced proton and antiproton. Specifically, it was shown within the scattering length approximation [47] and a distorted-wave Born approximation [35], that a calculation with a complex $S$-wave scattering length extracted from an effective-range analysis of $p \bar{p}$ scattering data [49] can reproduce the shape of of the $p \bar{p}$ mass distribution close to threshold. Reference [50] suggests that the enhancement is caused by a peripheral one-pionexchange potential.

A description of the experimental mass spectrum in terms of $p \bar{p}$ FSI effects does not contradict the existence of baryonium, which could also be present in the spectrum. A further investigation of the $p \bar{p}$ mass spectrum in different decay channels is thus desirable and is carried out in this work.

\subsection{New charmonium resonances}

In November 1974 two experiments, one at Brookhaven National Laboratory measuring the products of the collisions of $30 \mathrm{GeV}$ protons on a beryllium target, and the other one at SLAC's SPEAR $e^{+} e^{-}$storage ring, reported the simultaneous discovery of a new very narrow resonance with a mass of $3.1 \mathrm{GeV} / c^{2}[5]$. The $J / \psi$ discovery announcement was followed ten days later by the announcement of the observation of another narrow resonance $\psi^{\prime}$ at $3.68 \mathrm{GeV} / c^{2}$ at SPEAR [51]. Soon thereafter a $\psi^{\prime \prime}$ resonance was found at $3.77 \mathrm{GeV} / c^{2}$ by the DASP Collaboration at DESY's DORIS $e^{+} e^{-}$storage ring [52].

Discovery of the charmonium states revolutionized our understanding of the hadron spectroscopy by demonstration that they could be fairy well described by potential models with the qualitative features expected from QCD (see Table 1.2). The current state of knowledge of the charmonium system and transitions between them is summarized in Figure 1.2.

Only charmonium states with $J^{P C}=1^{--}$can be produced directly in $e^{+} e^{-}$collisions. The three prominent states $J / \psi(3100), \psi^{\prime}(3680)$ and $\psi^{\prime \prime}(3770)$ have been well established as the ${ }^{3} S_{1}, 2^{3} S_{1}$ and ${ }^{3} D_{1}$ states, respectively, with the latter being broad since it is just above the $D \bar{D}$ production threshold. Non-vector states such as the 
Table 1.2: Predicted and observed masses of $c \bar{c}$ states (in MeV) from Ref. [53]

\begin{tabular}{|c|c|c|c|c|c|c|c|}
\hline \multirow[t]{2}{*}{ State } & \multirow[t]{2}{*}{ Expt [20] } & \multicolumn{6}{|c|}{ Predictions } \\
\hline & & [17] & {$[54]$} & [55] & {$[56]$} & {$[57]$} & {$[58]$} \\
\hline $1^{3} \mathrm{~S}_{1}$ & $3096.87 \pm 0.04$ & 3098 & 3097 & 3104 & 3097 & 3096 & 3100 \\
\hline $1^{1} \mathrm{~S}_{0}$ & $2979.8 \pm 1.8$ & 2975 & 2980 & 2987 & 2979 & 2979 & 3000 \\
\hline $1^{3} \mathrm{P}_{2}$ & $3556.18 \pm 0.13$ & 3550 & 3507 & 3557 & 3557 & 3556 & 3540 \\
\hline $1^{3} \mathrm{P}_{1}$ & $3510.51 \pm 0.12$ & 3510 & 3486 & 3513 & 3511 & 3510 & 3500 \\
\hline $1^{3} \mathrm{P}_{0}$ & $3415.0 \pm 0.8$ & 3445 & 3436 & 3404 & 3415 & 3424 & 3440 \\
\hline $1^{1} \mathrm{P}_{1}$ & $3524.4 \pm 0.7$ & 3517 & 3493 & 3529 & 3526 & 3526 & 3510 \\
\hline $2^{3} \mathrm{~S}_{1}$ & $3685.96 \pm 0.09$ & 3676 & 3686 & 3670 & 3686 & 3686 & 3730 \\
\hline $2^{1} \mathrm{~S}_{0}$ & $3654 \pm 10$ & 3623 & 3608 & 3584 & 3618 & 3588 & 3670 \\
\hline $1^{3} \mathrm{D}_{3}$ & & 3849 & & 3884 & & 3815 & 3830 \\
\hline $1^{3} \mathrm{D}_{2}$ & & 3838 & & 3871 & & 3813 & 3820 \\
\hline $1^{3} \mathrm{D}_{1}$ & $3769.9 \pm 2.5$ & 3819 & & 3840 & & 3798 & 3800 \\
\hline $1^{1} \mathrm{D}_{2}$ & & 3837 & & 3872 & & 3811 & 3820 \\
\hline $2^{3} \mathrm{P}_{2}$ & & 3979 & & & & 3972 & 4020 \\
\hline $2^{3} \mathrm{P}_{1}$ & & 3953 & & & & 3929 & 3990 \\
\hline $2^{3} \mathrm{P}_{0}$ & & 3916 & & & & 3854 & 3940 \\
\hline $2^{1} \mathrm{P}_{1}$ & & 3956 & & & & 3945 & 3990 \\
\hline $3^{3} \mathrm{~S}_{1}$ & & 4100 & & & & 4088 & 4180 \\
\hline $3{ }^{1} \mathrm{~S}_{0}$ & & 4064 & & & & 3991 & 4130 \\
\hline
\end{tabular}




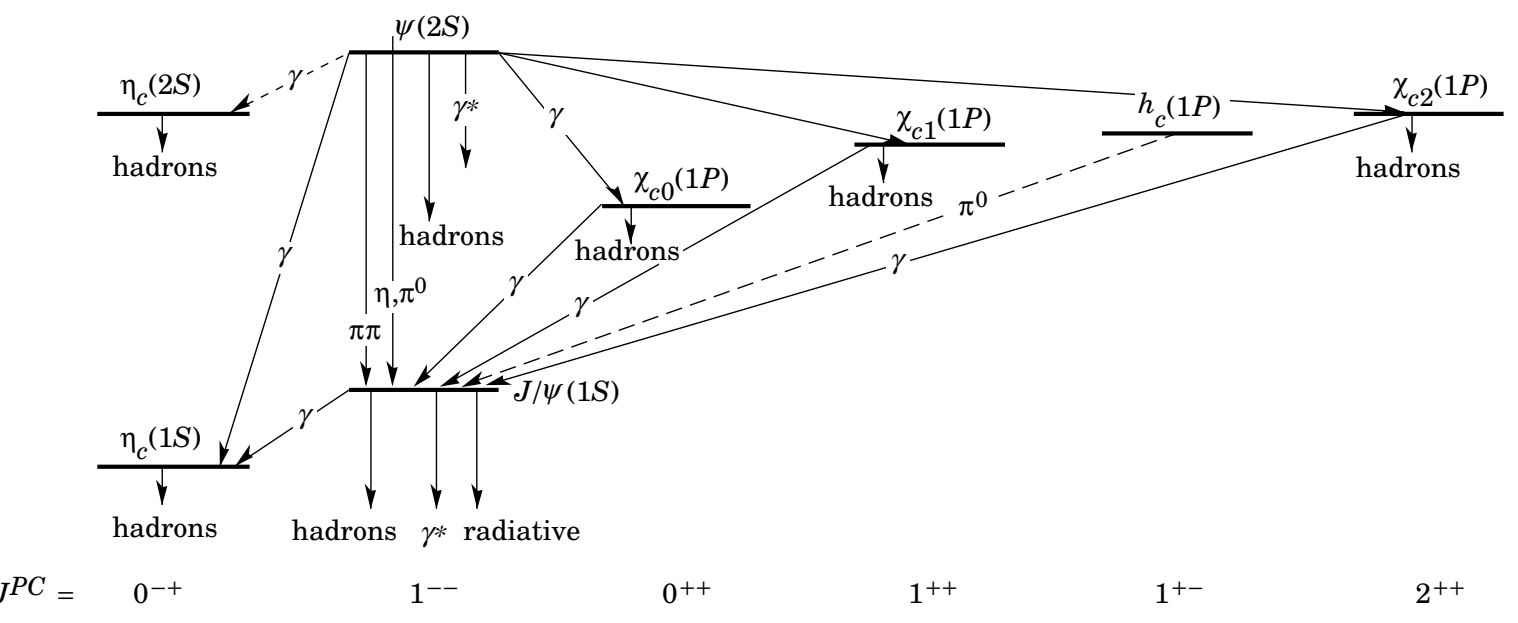

Figure 1.2: The current state of knowledge of the charmonium system and transitions from Ref. [20]. Uncertain transitions are indicated by dashed lines. The notation $\gamma^{*}$ refers to decay processes involving intermediate virtual photons, including decays to $e^{+} e^{-}$and $\mu^{+} \mu^{-}$.

${ }^{3} P_{J}$ (e.g. $\chi_{c J}$ ) and the ${ }^{1} S_{0}$ can be reached by E1 and M1 electromagnetic transitions from these states and thus can be observed in the radiative decays from the $2^{3} S_{1}$ level $\left(\psi^{\prime}(3700)\right)$.

The singlet states have been far more elusive. The $\eta_{c}\left(1^{1} S_{0}\right)$ state has been known for some time, seen in magnetic dipole $(M 1)$ transitions from both the $J / \psi$ and $\psi^{\prime}$. In contrast, a strong claim for observation of the $\eta_{c}^{\prime}\left(2^{1} S_{0}\right)$ state has only occurred recently, first with its observation in the decay $B \rightarrow K \eta_{c}^{\prime}, \eta_{c}^{\prime} \rightarrow K_{s} K^{+} \pi^{-}$by the Belle Collaboration [59] and its subsequent observation by Belle in the mass spectrum recoiling against $J / \psi$ in $e^{+} e^{-}$annihilation [60], and by CLEO [61] and BABAR [62] in $\gamma \gamma$ collisions. While the mass measurement by Belle was higher than expected by most quark potential models, the current world average [20] is in reasonable agreement with theory.

The elusive $h_{c}\left(1^{1} P_{1}\right)$ state of charmonium has been recently observed by CLEO [63, 64] via $\psi(2 S) \rightarrow \pi^{0} h_{c}$ with $h_{c} \rightarrow \gamma \eta_{c}$. Earlier $h_{c}$ sightings (see $[63,64]$ for references), based on $\bar{p} p$ production in the direct channel, include a few events at $3525.4 \pm 0.8 \mathrm{MeV}$ seen at the CERN ISR Experiment R704; a state at 3526.2 $\pm 0.15 \pm 0.2 \mathrm{MeV}$, decaying to $\pi^{0} \mathrm{~J} / \psi$, reported by E760 at Fermilab but not confirmed by E835; and a state at $3525.8 \pm 0.2 \pm 0.2 \mathrm{MeV}$, decaying to $\gamma \eta_{c}$ with $\eta_{c} \rightarrow \gamma \gamma$, reported by E835 with about 
a dozen candidate events [65]. The CLEO data yields $M\left(h_{c}\right)=(3524.4 \pm 0.6 \pm 0.4)$ $\mathrm{MeV}$.

Many charmonium states above the $D \bar{D}$ threshold have been seen recently. The $X(3872)$, discovered initially by Belle in $B$ decays [66], confirmed by BaBar [67] and also seen in hadronic production $[68,69]$, decays predominantly into $J / \psi \pi^{+} \pi^{-}$. Since it lies well above $D \bar{D}$ threshold but is narrower than the experimental resolution (a few $\mathrm{MeV}$ ), unnatural $J^{P}=0^{-}, 1^{+}$or $2^{-}$is favored. It has many features in common with an S-wave bound state of $\left(D^{0} \bar{D}^{* 0}+\bar{D}^{0} D^{* 0}\right) / \sqrt{2} \sim c \bar{c} u \bar{u}$ with $J^{P C}=1^{++}[70]$. The $X(3872)$ decays to $\rho J / \psi$ and $\omega J / \psi$ with roughly equal branching ratios. The analysis of angular distributions [71] in these decays [71] favors the $1^{++}$"molecular" assignment [72]. The detection of a small $\gamma J / \psi$ mode $\left(\sim 14 \%\right.$ of $\left.J / \psi \pi^{+} \pi^{-}\right)$[73] confirms the assignment of $C=+1$ and suggests some admixture of $c \bar{c}$ in the wave function.

Belle has reported a peak at $M(\omega J / \psi) \simeq 3940 \mathrm{MeV}$ in $B \rightarrow K \omega J / \psi$ decay [74]. This state is considered to be a candidate for an excited P-wave charmonium state, perhaps the $\chi_{c 1,2}^{\prime}\left(2^{3} P_{1,2}\right)[75]$. The corresponding $b \bar{b}$ states $\chi_{b 1,2}^{\prime}$ have been seen to decay to $\omega \Upsilon(1 S)[76]$.

A charmonium state distinct from this one, but also around $3940 \mathrm{MeV}$, is produced recoiling against $J / \psi$ in $e^{+} e^{-} \rightarrow J / \psi+X$ and is seen by Belle [77] to decay to $D \bar{D}^{*}$ + c.c., but not to $\omega J / \psi$. Since all lower-mass states observed in this recoil process have $J=0$ (the $\eta_{c}(1 S), \chi_{c 0}$ and $\eta_{c}^{\prime}(2 S)$; it was suggested [75] to identify this state as $\eta_{c}(3 S)$ (not $\chi_{c 0}^{\prime}$, which would decay to $\left.D \bar{D}\right)$.

Belle has recently reported a candidate for $\chi_{c 2}(3931)$ in $\gamma \gamma$ collisions [78], decaying to $D \bar{D}$. The angular distribution of $D \bar{D}$ pairs is consistent with that of $J=2, \lambda= \pm 2$ state. It has $M=3931 \pm 4 \pm 2 \mathrm{MeV}, \Gamma=20 \pm 8 \pm 3 \mathrm{MeV}$, and $\Gamma_{e e} \mathcal{B}(D \bar{D})=$ $0.23 \pm 0.06 \pm 0.04$, all of which are consistent with a $\chi_{c 2}^{\prime}$ state assignment [75].

Finally, BaBar reported a state $Y(4260)$ produced in the radiative return reaction $e^{+} e^{-} \rightarrow \gamma \pi^{+} \pi^{-} J / \psi$ and seen in the $\pi^{+} \pi^{-} J / \psi$ spectrum [79]. Its mass is consistent with being a $4 S$ level (see [80] for this interpretation) since it lies about $230 \mathrm{MeV}$ above the $3 S$ candidate (to be compared with a similar $4 S-3 S$ spacing in the $\Upsilon$ system). However, it could also be a hybrid state [81], as it lies roughly in the expected mass 
range $\left(\sim 4.33 \mathrm{GeV} / c^{2}\right)$, a $c s \bar{c} \bar{s}$ state [82], or an effect associated with $D_{s}^{*} \bar{D}_{s}^{*}$ threshold.

It is possible to study the charmonium states discussed above in the exclusive $B$ decays to charmonium, with charmonium decaying to $p \bar{p}$. This potentially gives access to all $J^{P C}$ quantum numbers of charmonia.

\subsection{Pentaquarks}

Dzierba et al. [83] and R.A. Schumacher [84] have prepared comprehensive surveys of experimental results on the pentaquark states. The information presented in this section is drawn for the most part from these articles.

Experimental searches for particles consisting of four quarks and an anti-quark $(q q q q \bar{q})$ have been ongoing since the early days of the quark model. The review by Hey and Kelly [85] discusses these early inconclusive searches for positive strangeness "Z" resonances, mostly in bubble chamber experiments.

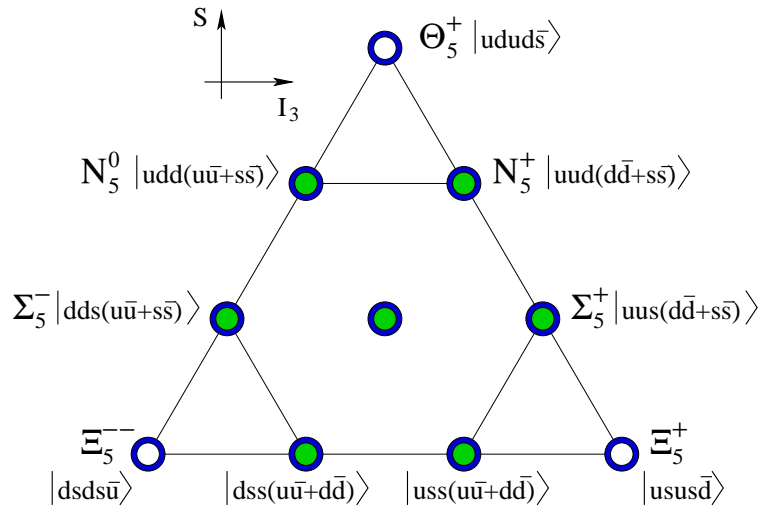

Figure 1.3: The predicted antidecuplet [86] of pentaquark baryons indicating, in particular, the states $\Theta^{+}=u u d d \bar{s}, \Xi_{5}^{--}=d d s s \bar{u}$ and $\Xi_{5}^{0}=u d s s \bar{d}$. Evidence for these states have been presented as well as for a $\Theta_{c}=u u d d \bar{c}$. Other searches for these states, however, have yielded null results.

Recent interest in pentaquark states originates from a prediction made by Diakonov et al. [86] in the context of a chiral-quark soliton model for an anti-decuplet of pentaquark states (see Figure 1.5). It predicts a narrow $\left(\Gamma_{\Theta^{+}} \approx 10 \mathrm{MeV}\right) u u d d \bar{s}$ state, the $\Theta^{+}$, close to $M=1530 \mathrm{MeV} / c^{2}$. The same model predicts seven non-exotic pentaquark baryons which could behave like ordinary $N^{*}$ states or hyperons. It also predicts cascade-like $S=-2$ states near $2070 \mathrm{MeV} / c^{2}$, two of which the $\Xi_{5}^{--}$and the $\Xi_{5}^{+}$had the charge-flavor-exotic structures $d d s s \bar{u}$ and $u u s s \bar{d}$, respectively. In this model pentaquarks emerge as rotational excitations of the soliton, with $J^{P}=\frac{1}{2}^{+}$. 
Evidence for the $\Theta^{+}$state has been reported by several experiments [87, 88, 89, 90, 91, 92, 93, 94, 95, 96, 97, 98]. Also $\Xi_{5}^{--}$[99], an anti-charm pentaquark baryon $\Theta_{c}(u u d d \bar{c})[100]$ and $\Theta^{++}[101]$, an isospin partner of $\Theta^{+}$have been seen by one experiment each. Only $\Theta^{+}$and $\Theta^{++}$results are discussed below.

Table 1.5 lists the experiments claiming evidence for pentaquark states. There are ten experiments claiming a $\Theta^{+}$, a state with $S=+1$, a mass of about $1.54 \mathrm{GeV} / c^{2}$ and a width consistent with being less than the mass resolutions of the experiments. The state is observed through its decay into either $K^{+} n$ or $K_{S}^{0} p$.

Table 1.3: Experimental results with positive signals for $\Theta^{+}$state. Please see the text regarding the final state neutron in the LEPS, CLAS and SAPHIR experiments.

\begin{tabular}{lllll}
\hline Experiment & Reaction & Mode & Ref. & Null-result [Ref.] \\
\hline LEPS(1) & $\gamma C_{12} \rightarrow K^{+} K^{-} X$ & $K^{+} n$ & {$[87]$} & CLAS [102] \\
LEPS(2) & $\gamma d \rightarrow K^{+} K^{-} X$ & $K^{+} n$ & {$[88]$} & CLAS [102] \\
CLAS(d) & $\gamma d \rightarrow K^{+} K^{-}(n) p$ & $K^{+} n$ & {$[89]$} & CLAS [102] \\
CLAS(p) & $\gamma p \rightarrow K^{+} K^{-} \pi^{+}(n)$ & $K^{+} n$ & {$[90]$} & \\
SAPHIR & $\gamma p \rightarrow K_{S}^{0} K^{+}(n)$ & $K^{+} n$ & {$[91]$} & CLAS [103] \\
COSY & $p p \rightarrow \Sigma^{+} K_{S}^{0} p$ & $K_{S}^{0} p$ & {$[92]$} & \\
JINR & $p\left(C_{3} H_{8}\right) \rightarrow K_{S}^{0} p X$ & $K_{S}^{0} p$ & {$[93]$} & \\
SVD & $p A \rightarrow K_{S}^{0} p X$ & $K_{S}^{0} p$ & {$[94,104]$} & {$[105,106,107,108]$} \\
DIANA & $K^{+} X e \rightarrow K_{S}^{0} p(X e)^{\prime}$ & $K_{S}^{0} p$ & {$[95]$} & BELLE [109] \\
$\nu$ BC & $\nu A \rightarrow K_{S}^{0} p X$ & $K_{S}^{0} p$ & {$[96]$} & \\
HERMES & $q u a s i-r e a l$ photoproduction & $K_{S}^{0} p$ & {$[97]$} & BABAR [110] \\
ZEUS & $e p \rightarrow K_{S}^{0} p X$ & $K_{S}^{0} p$ & {$[98]$} & H1 [111],BABAR [112] \\
\hline
\end{tabular}

The first five reactions listed in Table 1.5 use a photon probe of relatively low energy, a few $\mathrm{GeV}$, and the reported pentaquark candidate is observed in the $K^{+} n$ mode. The first sighting of the $\Theta^{+}$is from LEPS at Spring8 $[87,88]$ followed shortly thereafter by CLAS at Jefferson Lab $[89,90]$. The SAPHIR experiment is also a lowenergy photon experiment [91]. The final state neutron in these five experiments is undetected. In the LEPS experiment the assumed $K^{+} n$ effective mass is actually the missing mass recoiling against the $K^{-}$. In the CLAS(d) experiment the assumption is that the final state proton, which is detected, is the spectator nucleon and the $K^{+} n$ 
effective mass is the mass recoiling against the $K^{-} p$ system.

After repeating the measurement on deuterium with six times higher statistics [102], CLAS observes no $\Theta^{+}$peak in the $\gamma d \rightarrow p K^{+} K^{-}(n)$ reaction. The previous CLAS result, when fit with a luminosity-scaled background shape from the higher statistics run, is reduced in significance to $\sim 3 \sigma$. Albeit the kinematic conditions are not the same as those at LEPS, there is no good strong experimental evidence left to suppose the existence of a $\Theta^{+}$produced in these channels.

In SAPHIR, the neutron is inferred by kinematic fitting. In the same reaction with the same kinematics but with much higher statistics, no $\Theta^{+}$signal is found by CLAS [103].

In the CLAS(p) experiment the neutron is inferred from missing mass. The cuts are chosen to enhance diffractive production of a high mass non-strange nucleon resonance which could then decay to a $K^{-}$and a $\Theta^{+}$. A $7.8 \sigma$ signal is reported. It is perhaps the most convincing remaining candidate at the moment.

The COSY experiment uses a low-momentum proton beam [92] spanning the momentum range from 2.85 to $3.3 \mathrm{GeV} / c$. The JINR result comes from an analysis of collisions in a propane bubble chamber exposed to a $10 \mathrm{GeV} / c$ proton beam. The SVD experiment [94] at IHEP studies $p A$ collisions at $70 \mathrm{GeV} / c$. Their initial report [94] is supported by a more recent [104] detailed analysis which increased their pentaquark signal by a factor of about eight. This measurement must be compared to the negative results from WA89 in the scattering of a $340 \mathrm{GeV} / c \Sigma^{-}$beam from carbon and copper [105], SPHINX at IHEP, with $70 \mathrm{GeV}$ protons on carbon [106], HyperCP at Fermilab [107], with $800 \mathrm{GeV}$ protons on a carbon target, and finally HERA-B [108] with the interaction at $41.6 \mathrm{GeV}$ c.m. energy of protons on several nuclear targets. Thus, despite the recent positive result reported by SVD-2, the evidence against the production of $\Theta^{+}$pentaquarks in high energy hadronic production is strong.

Both the DIANA and $\nu \mathrm{BC}$ groups re-analyzed old data from a liquid xenon bubble chamber (in the case of DIANA) [95] and the CERN BEBC and the FNAL 15-foot chamber (in the case of $\nu \mathrm{BC}$ ) [96] - the former using a low-energy $K^{+}$beam, the latter neutrinos. The HERMES experiment at DESY found evidence for the $\Theta^{+}$with quasi-real photons [97] while ZEUS claims evidence [98] for the $\Theta^{+}$in ep collisions. 
The HERMES measurement can be contrasted with the much higher statistics null measurements from the BaBar Collaboration at SLAC [110] in both electro- and hadro-production in the material of the BABAR detector. The ZEUS results are being contradicted by the null-measurement from H1 [111] and BABAR [112] experiments.

The evidence for the $\Theta^{+}$presented in references $[87,88,89,90,91]$ is in the $K^{+} n$ mode, which is manifestly flavor-exotic while the other experiments report the $\Theta^{+}$in the $K_{S}^{0} p$ mode, which is a linear combination of $S=+1$ and $S=-1$.

If the $\Theta$ pentaquark were an isovector, not the isoscalar predicted in most models, then other charge states should exist, such as a $\Theta^{++}$. The decay of this state to $K^{+} p$ is especially easy to look for. Several of these experiments study the $K^{+} p$ spectrum and find no evidence for a $\Theta^{++}$, thus concluding that $I=1 / 2$ for the $\Theta^{+}$. As it is not observed in the $p K^{+}$scattering it is possible to infer an upper limit on its width. The $p K^{+}$cross section is nearly purely elastic in the region of interest so a resonance would follow the Breit-Wigner form, with a peak cross section of about $25 \mathrm{mb}$ if the resonance is at $1.7 \mathrm{GeV} / \mathrm{c}^{2}$ and even larger if the mass is somewhat lower. The cross section has been measured to be about $12 \mathrm{mb}$ at center-of-mass energies spaced by about $15 \mathrm{MeV}$ [20], so its width would need to be considerably less than $15 \mathrm{MeV}$ to have escaped detection. Whereas many non-sightings have been mentioned in the pentaquark literature, a recent result from the STAR Collaboration at RHIC claims [101] a narrow state decaying to $K^{+} p$ in deuteron-gold collisions at $200 \mathrm{GeV}$ $N N$ c.m. energy, with a $4.2 \sigma$ significance. The state sits atop a very large but smooth background, but unfortunately also sits next to an equally large bump that is attributed to $K / \pi$ particle identification errors. Confirmation of this structure and its interpretation are clearly needed.

After an initial flurry of positive reports for these states, null results started to dominate the field. First non-observations of these states have often come from reaction channels very different from the positive evidence channels, making the comparison difficult. Non-observations of the $\Theta^{+}$were reported for $J / \psi$ decays involving $\Theta^{+} \rightarrow K_{S}^{0} p$ from BES [113], for $p \bar{p}$ collisions from CDF [114], and from events in $e^{+} e^{-}$collisions at the $Z$ pole from ALEPH [115]. The significance of these results in relation to the positive observations at low energies is difficult to estimate, since 
the production mechanism of exotic pentaquarks is, well, exotic. Nevertheless, these results add some weight to the conclusion that these states have in fact not been seen. Recent high-statistics repetitions of experiments, wherein there were positive observations, have not convincingly reproduced any of the positive results. Most of the unconfirmed positive sightings suffer from low statistics and large backgrounds.

Following the observation of the charmless baryonic three-body $B$ decays of the type $B \rightarrow p \bar{p} K$, it was suggested that this decay might include events of the form $B \rightarrow \Theta \bar{p}$. Both states with $\Theta^{+} \rightarrow p K_{S}^{0}$ and its isovector partner $\Theta^{++} \rightarrow p K^{+2}$ would be accessible [117] with limited statistics but very little background. The results of these null searches are reported in the current work.

\section{$1.6 \quad B$ decays}

$B$ mesons are heavy enough to decay into a baryon-antibaryon pairs while conserving the baryon number. Since the baryons are heavy, it was originally assumed that the decays with many additional mesons are suppressed by phase-space limitations and the need to produce an extra quark-antiquark pair. Inspired by the claim of the observation of the decay modes $B^{+} \rightarrow p \bar{p} \pi^{+}$and $B^{0} \rightarrow p \bar{p} \pi^{+} \pi^{-}$by ARGUS [118] in the late 1980s, baryonic $B$ decays were studied extensively with the focus on the tree-dominated two-body decay modes, e.g. the "charmful" decays $B \rightarrow \Lambda_{c} \bar{n}, \Sigma_{c} \bar{N}$, and "charmless" ones $B \rightarrow p \bar{p}, \Lambda \bar{\Lambda}$. Several authors have attempted to calculate exclusive two-body decay rates into baryons $[119,120,121,122,123]$. The theoretical predictions are model-dependent and vary quite drastically with many of the earlier model predictions being too large compared to experimental limits [20], see Table 1.4. Only recently a few charmful modes have been detected $[124,125]$, more than an order of magnitude below the model predictions. Two of the two-body decay modes, $B^{0} \rightarrow \Lambda_{c} \bar{p}$ and $B^{+} \rightarrow \Lambda(1520) K^{+}$, will be discussed in the current work.

It was pointed out in 1998 by Dunietz [126] and later by Hou and Soni [127], that the smallness of the two-body baryonic decays has to do with the large energy

\footnotetext{
${ }^{2} \Theta^{++}$can also be an $I=1, I_{3}=1$ pentaquark, which is a member of the baryon 27-plet and with quark content uuuds. It is predicted to lie in the region $1.43-1.70 \mathrm{GeV} / \mathrm{c}^{2}[116]$.
} 
Table 1.4: Predictions of the branching ratios $\left(\times 10^{-6}\right)$ for some two-body baryonic $B$ decays. Branching ratios denoted by " $\dagger$ " are calculated only for the parity-conserving part. We have normalized the branching ratios to $\left|V_{u b} / V_{c b}\right|=0.085$.

\begin{tabular}{|c|c|c|c|c|c|c|}
\hline & Ref. [119] & Ref. [120] & Ref. [121] & Ref. [122] & Ref. [123] & Expt. [20] \\
\hline $\bar{B}^{0} \rightarrow p \bar{p}$ & 4.2 & 1.2 & 7.0 & $2.9-27$ & $0.11^{\dagger}$ & $<1.2$ \\
\hline $\bar{B}^{0} \rightarrow \Lambda \bar{\Lambda}$ & & & 0.2 & & $0^{\dagger}$ & $<1.0$ \\
\hline$B^{-} \rightarrow p \bar{\Delta}^{--}$ & 150 & 0.29 & 320 & $2.4-8.7$ & 1.4 & $<150$ \\
\hline$B^{-} \rightarrow \Lambda \bar{p}$ & & $\lesssim 3$ & & & $0.22^{\dagger}$ & $<2.2$ \\
\hline $\bar{B}^{0} \rightarrow \Lambda_{c} \bar{p}$ & & 400 & 1000 & $1700-1900$ & & $22 \pm 8$ \\
\hline $\bar{B}^{0 /+} \rightarrow \bar{\Lambda}_{c} \Xi_{c}$ & & 1000 & & $1200-1800$ & & $\sim 1200(4800)$ \\
\hline
\end{tabular}

release. This feature is easy to understand by studying the Dalitz plot for treedominated processes. Due to the $V-A$ nature of the $b \rightarrow u d \bar{u}$ process, the invariant mass of the diquark $u d$ peaks at the highest possible values in the Dalitz plot for $b \rightarrow u d \bar{u}$ transition. If the $u d$ forms a nucleon, then the very massive $u d q$ objects will tend to form a highly excited baryon state such as $\Delta$ and $N^{*}$ and be seen as $N n \pi(n \geq 1)$. This explains the non-observation of the $N \bar{N}$ final states and why the three body modes of type $N \bar{N} \pi(\rho)$ are favored. In the three-body baryonic decay, $B \rightarrow \mathcal{B B} M$, the emission of the meson $M$ will carry away energy in such a way that the $\mathcal{B} \overline{\mathcal{B}}$ invariant mass becomes smaller and hence it is relatively easier to fragment into the baryon-antibaryon pair.

The first charmful baryonic mode, $D^{*+} n \bar{p}$, with the branching fraction on the order of $10^{-3}$, was discovered by CLEO in 2001 [41]. The first charmless baryonic three-body mode $p \bar{p} K^{+}$was observed a year later by Belle [40]. Observations of other modes soon followed [128]. This work will concentrate on studying both penguindominated modes involving $b \rightarrow s$ transition $\left(B \rightarrow p \bar{p} K^{+/ 0(*)}\right)$ and tree-dominated mode $\left(B^{+} \rightarrow p \bar{p} \pi^{+}\right)$.

The Feynman diagrams for $B^{+} \rightarrow p \bar{p} K^{+(*)}$ decay are shown in Figure 1.4. The leading diagrams [122] are a penguin diagram and a doubly Cabibbo-KobayashiMaskawa (CKM) [130] suppressed tree diagram shown in Figure 1.4(a,b). There is also 
(a)

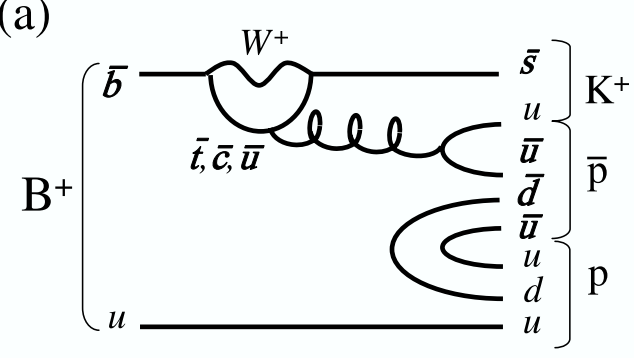

(b)

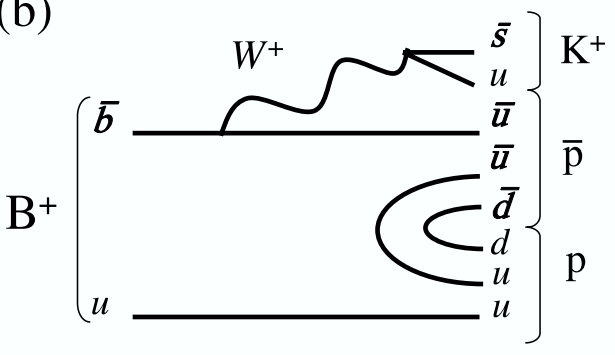

(c)

Figure 1.4: Idealization [129] of the main Feynman diagrams for the non-resonant $B^{+} \rightarrow p \bar{p} K^{+}$decay: (a) leading penguin diagram, (b) leading tree diagram (external $W^{+}$-emission), (c) Okubo-Zweig-Iizuka-suppressed penguin diagram, (d,f) colorsuppressed penguin diagrams with an internal gluon-emission, (e) an internal $W^{+}$ emission and, (g) a $W^{+}$-annihilation.

(d)

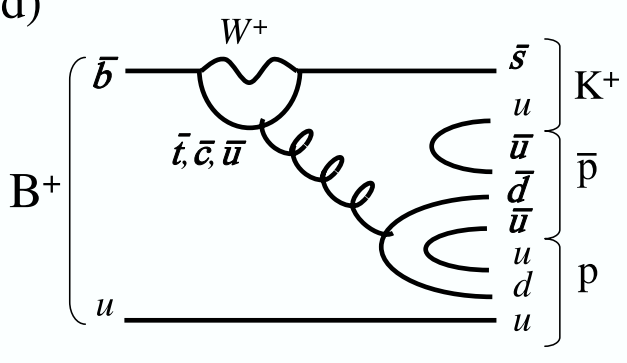

(f)

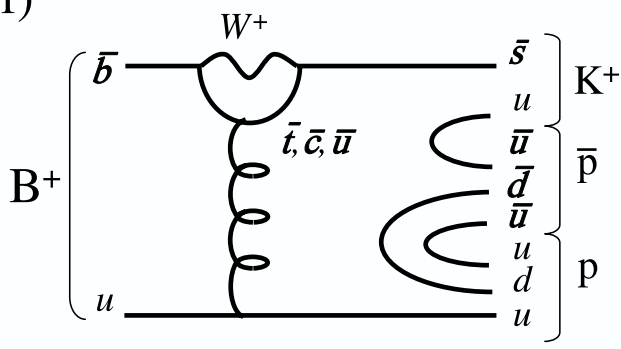

(e)

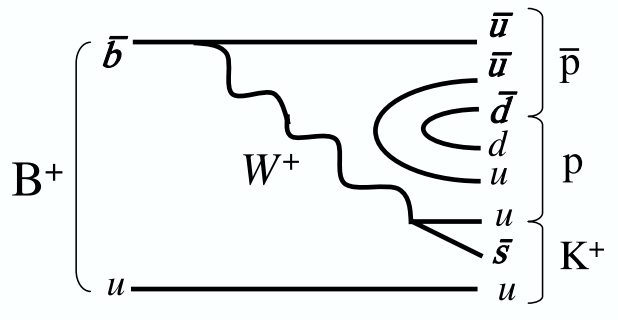

(g)

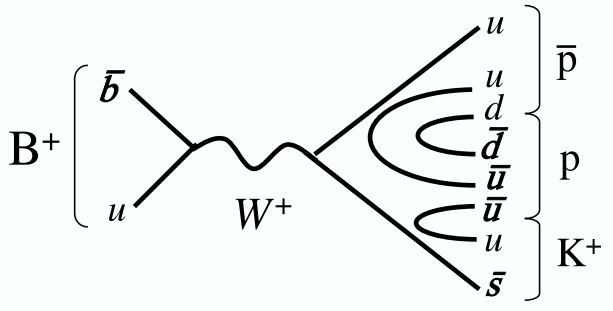



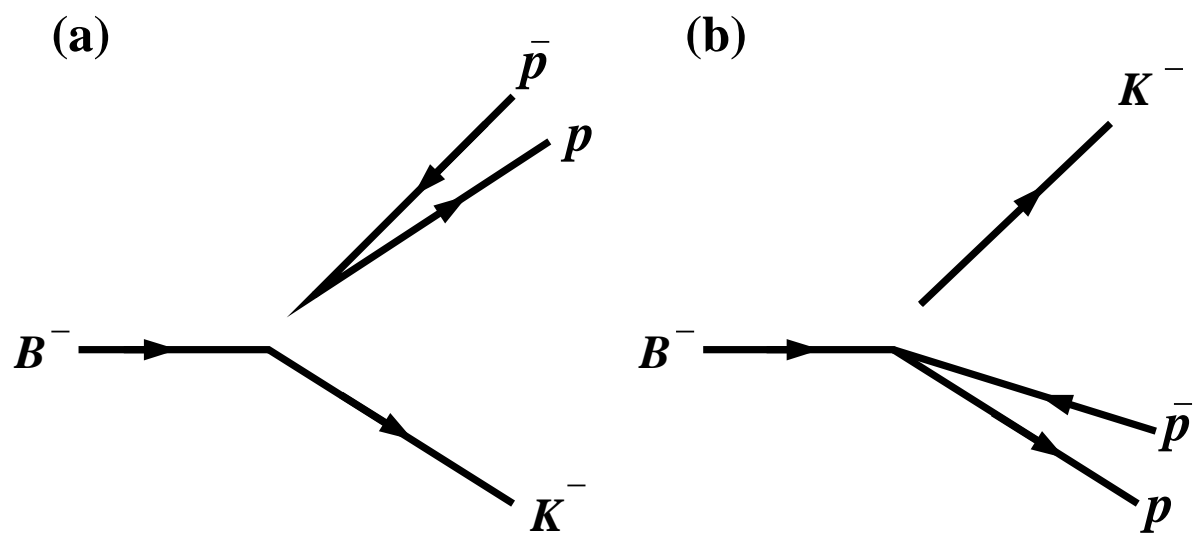

Figure 1.5: (a) The current-produced $(\mathcal{J})$ and (b) transition $(T)$ diagrams for $B^{+} \rightarrow$ $p \bar{p} K^{+}$decay from Ref. [28].

an Okubo-Zweig-Iizuka-suppressed penguin diagram shown in Figure 1.4(c), where the $p \bar{p}$ pair is created through a pair of gluons (or a gluonic resonance). There are four additional color-suppressed diagrams [122]: two tree diagrams with an internal $W^{+}$-emission and a $W^{+}$-annihilation (Figure 1.4(e,g)) and two penguin diagrams with an internal gluon-emission (Figure $1.4(\mathrm{~d}, \mathrm{f})$ ) which are expected to be small. All the diagrams shown in Figure 1.4 with the exception of the leading tree diagram and the $W^{+}$-exchange diagram ${ }^{3}$ (Figure $1.4(\mathrm{~b}, \mathrm{~g})$ ) are valid for the $B^{0} \rightarrow p \bar{p} K^{0(*)}$ decay. To obtain the Feynman diagrams for the $B^{+} \rightarrow p \bar{p} \pi^{+}$mode, one needs to replace the $\bar{s}$ quark in Figure 1.4 by $\bar{d}$; in this case the leading diagram will be a tree diagram shown in Figure 1.4(b).

Since baryonic $B$ decays involve two baryons, precise theoretical calculations are extremely complicated. In recent years the factorization approach has been successfully applied to calculation of the charmless baryonic three-body decay branching fractions and in prediction of the decay dynamics. The three-body baryonic $B$ decay matrix element is separated into two parts as shown in Figure 1.5: a current-produced baryon-pair $(\mathcal{J})$ part together with a $B$ recoil meson transition part, $\langle B|\mathcal{J}| K\rangle\langle p \bar{p}\rangle$ (eg. Figure 1.4(c)), and a B-to-baryon-transition $(T)$ part together with a current

\footnotetext{
${ }^{3} W^{+}$-exchange diagram is valid for neutral $B$ decay, while $W^{+}$-annihilation for the charged $B$ decay.
} 
Table 1.5: Summary of the experimental and theoretical values for the branching fractions $\left(\times 10^{-6}\right)$ of $B \rightarrow p \bar{p} h$.

\begin{tabular}{||c||c||c|c||}
\hline Mode & {$[131,132]$} & {$[122]$} & {$[28]$} \\
\hline$p \bar{p} K^{+}$ & $5.30_{-0.39}^{+0.45} \pm 0.58$ & 4.0 & $0.2-4.8$ \\
\hline$p \bar{p} K^{*+}$ & $10.3_{-2.8}^{+3.6+1.3}$ & 2.3 & - \\
\hline$p \bar{p} K_{S}^{0}$ & $1.20_{-0.51}^{+0.62} \pm 0.39$ & 0.1 & $0.5-3.6$ \\
\hline$p \bar{p} K^{* 0}$ & $<7.6,90 \%$ CL & 0.05 & - \\
\hline$p \bar{p} \pi^{+}$ & $3.06_{-0.62}^{+0.73} \pm 0.37$ & $1-2$ & $1.9-2.0$ \\
\hline
\end{tabular}

produced recoil-meson part, $\langle B|T| p \bar{p}\rangle\langle K\rangle$ (eg. Figure 1.4(b)). A factorization approach is generally used in the literature for the evaluation of the current-produced amplitude [122, 28]. Currently two approaches were used for the evaluation of the three-body matrix element in the transition process: the pole model [122] or factorizing amplitude into a current-produced meson and a $B$ to baryonic pair transition amplitude [28]. A summary of the experimental situation and theoretical predictions prior to this work are given in Table 1.5.

In Ref. [122], Cheng and Yang use the factorization approach for calculation of the current-produced $(\mathcal{J})$ amplitude and a simple pole model for calculation of the transition $(T)$ part (so-called "pole model"). They assume, for example, in $B^{+} \rightarrow$ $p \bar{p} K^{+}$decay, a strong process $B^{+} \rightarrow \bar{\Lambda}_{b}^{*}, \bar{\Sigma}_{b}^{*} p$, followed by a weak $\bar{\Lambda}_{b}^{*}, \bar{\Sigma}_{b}^{*} \rightarrow \bar{p} K^{+}$decay. This model gives values close to experimental results for the $B^{+} \rightarrow p \bar{p} K^{+}$decay rate by using a monopole $\left(\Lambda_{b}\right) q^{2}$ dependence, and an order of magnitude lower result assuming both $\Lambda_{b}$ and $\Sigma_{b}$ poles. In turn, the $B^{0} \rightarrow p \bar{p} K^{0}$ rate is expected to be suppressed to $\sim 10^{-7}$ due to the absence of the $\Lambda_{b}$ pole. The $B^{+} \rightarrow p \bar{p} K^{*+}$ and $B^{0} \rightarrow p \bar{p} K^{* 0}$ modes are expected to be smaller as some of the penguin contributions are absent.

In Ref. [28], C.K. Chua et al. use the factorization approach for both current and transition parts (so-called "form-factor model"). They apply SU(3) relations and QCD counting rules [133] on baryon form factors. In particular the transition part is described by three form factors: two for chiral conserving parts $\left(F_{A}\right.$ and $\left.F_{V 5}\right)$ with the interacting quark spins parallel and anti-parallel to the proton, and a chiral flipping 
part $F_{P}$, with only the parallel spins. The form factors are parametrized as follows:

$$
F_{A, V 5}=\frac{C_{A, V 5}}{m_{p \bar{p}}^{3}}, F_{P}=\frac{C_{P}}{m_{p \bar{p}}^{4}},
$$

where $C_{A, V 5, P}$ are coefficients to be determined experimentally. The $p \bar{p} \pi^{+}, p \bar{p} K^{+}$ and $p \bar{p} K^{0}$ modes are dominated by transition contributions, with the current contribution being significant for the last two modes. Under factorization hypothesis all three modes have the same form factors and one can use the form factor parameters obtained from the $p \bar{p} \pi^{+}$mode (which has only a transition contribution) in the calculation of the amplitude of the latter two modes. Only the cases when one of the form factors dominates are considered. The authors predict $B^{+} \rightarrow p \bar{p} K^{+}$rate close to the measured one and obtain an $m_{p \bar{p}}$ threshold enhancement from their calculation. They also predict that the enhancement in $B^{+} \rightarrow p \bar{p} K^{+}\left(\propto 1 / m_{p \bar{p}}^{2}\right)$ decreases slower with $m_{p \bar{p}}$ than that in $B^{+} \rightarrow p \bar{p} \pi^{+}\left(\propto 1 / m_{p \bar{p}}^{3,4}\right)$, which can be verified experimentally. For the $B^{0} \rightarrow p \bar{p} K^{0}$ mode the current-induced part is identical to that in $B^{+} \rightarrow p \bar{p} K^{+}$and the transition part for $\mathcal{B}_{\mathcal{T}}\left(B^{0} \rightarrow p \bar{p} K^{0}\right)$ can be $\approx \mathcal{B}_{\mathcal{T}}\left(B^{+} \rightarrow p \bar{p} K^{+}\right), \ll \mathcal{B}_{\mathcal{T}}\left(B^{+} \rightarrow p \bar{p} K^{+}\right)$or $\gg \mathcal{B}_{\mathcal{T}}\left(B^{+} \rightarrow p \bar{p} K^{+}\right)$if $C_{A}, C_{P}$ or $C_{V 5}$ contributions are dominant, respectively. The authors also compare $p \bar{p} h$ modes with the familiar two-meson $B$ decays. For the $B \rightarrow p \bar{p}$ transition part, the analogous transitions are $B^{+} \rightarrow \pi^{0}, \rho^{0}$ (or isospin related $B^{0} \rightarrow \pi^{-}, \rho^{-}$). In principle all the two body modes mentioned above, except $B^{+} \rightarrow \pi^{0} \pi^{+}$(which have the cancellation of strong penguin amplitude in $B^{+} \rightarrow \pi^{0}$ and $B^{+} \rightarrow \pi^{+}$) have similar transition terms. For the current-produced part, similar terms can be found in $B^{+} \rightarrow \pi^{0} \pi^{+}, \pi^{0} K^{+}, \rho^{0} \pi^{+}$, and $\rho^{0} K^{+}$decay amplitudes. However there are additional terms in there as the isosinglet currents are non-vanishing, in contrast to the two-body modes, where $\pi^{0}\left(\rho^{0}\right)$ are members of an isotriplet and can not be produced via isosinglet current.

One can compare the two models using the $B^{+} \rightarrow p \bar{p} K^{+}$mode. In particular the $m_{p \bar{p}}$ spectrum in the pole model peaks around $m_{p \bar{p}} \approx 2.5 \mathrm{GeV} / c^{2}$, while in the form-factor model there is a sharper peak at lower $m_{p \bar{p}}$ (around $2 \mathrm{GeV} / c^{2}$ ). Also, in the pole model one expects peaking behavior toward large $m_{p K^{-}}$(due to $\Lambda_{b}$ pole), while in the form-factor model there is no structure in the $m_{p K^{-}}$spectrum. The rate 
of $B^{0} \rightarrow p \bar{p} K^{0}$ is expected to be $\sim 10^{-7}$ in the pole model, while in the form-factor model it can be as large as $B^{+} \rightarrow p \bar{p} K^{+}$.

One of the interesting features observed in the three-body baryonic $B$ decays is a low-mass $m_{p \bar{p}}$ enhancement. As discussed in Section 1.3 there are explanations for this feature as a resonance or a result of the quark fragmentation process [46]. It was suggested [46] that one can distinguish the fragmentation or below-the-threshold resonance mechanisms by studying the distribution of events in the Dalitz plot. If the $p \bar{p}$ system is produced independently of the $K^{+}$through a tree diagram with an external $W^{+}$-emission (Figure 1.4(b)) or a penguin with an external gluon-emission (Figure 1.4(c)), i.e. the $p \bar{p}$ quark lines are not associated with the $\bar{s}$ or $u$ quarks in the $K^{+}$, then the distributions $m_{p K^{+}}$and $m_{\bar{p} K^{+}}$should be identical. If the $u$ quark in the $K^{+}$is associated with a $\bar{u}$ quark in a $\bar{p}$ (Figure 1.4(a)), larger values of $m_{p K^{+}}$are favored over those of $m_{\bar{p} K^{+}}$[46]. Thus a study of the Dalitz plot provides insight not only into the dominant mechanism of this decay but also into whether the penguin or the tree amplitude is dominant.

By removing extra quark lines from Figures $1.4(\mathrm{a}, \mathrm{b}, \mathrm{e})$ one can relate $B^{+} \rightarrow p \bar{p} K^{+}$ to corresponding penguin and tree diagrams of $B^{+} \rightarrow K^{+} \pi^{0}$. After observation of the direct CP violation in $B^{0} \rightarrow K^{+} \pi^{-}[134]$ and its non-observation in $B^{+} \rightarrow K^{+} \pi^{0}$ [135], it would also be interesting to study the charge asymmetry in the $B^{+} \rightarrow p \bar{p} K^{+}$system.

In this work, we present a comprehensive study of the pattern in the $B \rightarrow$ $p \bar{p} h$ branching fractions, the nature of the $m_{p \bar{p}}$ spectrum, resonant substructures and an evaluation of CP asymmetries in these decays. 


\section{Chapter 2}

\section{The BABAR Detector and Data}

The BABAR detector was designed to study the decays of $B$ mesons produced at the PEP-II asymmetric-energy electron-positron collider. Both collider and detector are optimized for their primary purpose: the study of time-dependent $\mathrm{CP}$ asymmetries in neutral B meson decays. Nonetheless, the detector is sufficiently versatile to allow a full range of $B$ physics measurements to be made. This section gives a brief description of the PEP-II collider and its performance, and details the components of the BABAR detector relevant for current work $[136]^{1}$.

\subsection{The PEP-II Collider}

The PEP-II $B$-factory is an asymmetric $e^{+} e^{-}$collider designed to operate at a luminosity of $3 \times 10^{33} \mathrm{~cm}^{-2} \mathrm{~s}^{-1}$ and above, at a center-of-mass energy of $10.58 \mathrm{GeV}$, which is the mass of the $\Upsilon(4 S)$ resonance. As this resonance decays almost exclusively to $B^{0} \bar{B}^{0}$ and $B^{+} B^{-}$pairs, it provides an ideal laboratory for the study of $\mathrm{B}$ mesons.

The collider itself consists of a pair of storage rings which collide a $9.0 \mathrm{GeV}$ electron beam with a $3.1 \mathrm{GeV}$ positron beam. The electron and positron beams are stored with respective currents of over $1.0 \mathrm{~A}$ and $1.5 \mathrm{~A}$, in 1658 bunches approximately $120 \mu \mathrm{m} \times 6 \mu \mathrm{m} \times 9 \mathrm{~mm}$ in size $(\mathrm{x}, \mathrm{y}, \mathrm{z})$. Dipole and quadrupole magnets are used to steer and focus the beams, respectively, and bring them into collision; the centroid of the

\footnotetext{
${ }^{1}$ Unless otherwise specified, all information in this chapter is derived from this reference.
} 
overlap of the beam profiles - the beam-beam interaction point, or IP - is monitored over time relative to the $B A B A R$ detector. The relative energies of the two beams are such that the Lorentz boost relative to the lab is $\beta \gamma=0.56$.

\subsection{The BABAR Detector}

The study of B decays typically involves either partial or full reconstruction of the decay chain of the $\mathrm{B}$ meson down to the final-state particles: charged hadrons $(\pi, K$,

$p)$, charged leptons $(e, \mu)$, and photons. Intermediate states in the decay chain are reconstructed as composites of the final-state particles. Reconstruction of the decay chain and kinematics of the decay is rarely unambiguous, and optimal reconstruction requires good knowledge of the following:

- Momentum and charge of charged tracks,

- Particle identification of charged tracks,

- The energy and direction (momentum) of photons.

The BABAR detector consists of the five sub-detector components shown in Figure 2.1, each of which provides complementary information about the final-state products of the B decay. From the innermost to outermost, the sub-detectors, together with their primary tasks, are:

- Silicon Vertex Detector (SVT): Precise tracking of charged particles near the interaction region, and measurement of energy loss $(d E / d X)$.

- Drift Chamber (DCH): Precise measurement of momentum and trajectory of charged particles, and measurement of energy loss $(d E / d X)$.

- Ring-imaging Cerenkov Detector (DIRC): Charged particle identification, particularly $\pi / K / p$ discrimination.

- Electromagnetic Calorimeter (EMC): Position and energy measurement of photons and leptons. Hadron rejection, and particularly electron identification. 


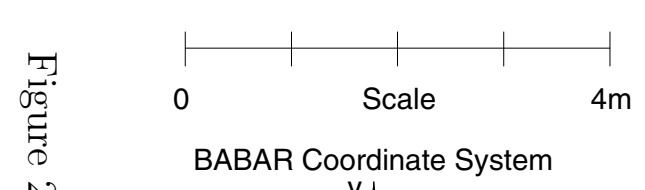

Detector $\mathbb{E}$

Instrumented
I.P.

BABAR Coordinate System

N

5 Cryogenic Chimney $\bar{z}$

\section{Cherenkov}

Detector

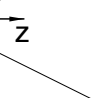

$+1149+-1015-1749$

-

Superconducting

Coil

Electromagnetic

Calorimeter (EMC)

Drift Chamber (DCH)

Silicon Vertex

Tracker (SVT)

:

ค Magnetic Shield

for DIRC

$\stackrel{\overbrace{}}{\stackrel{5}{\circ}}$

음.

$\stackrel{0}{\circ}$

$\stackrel{0}{\oplus}$

कृ

何

हJ

$\frac{0}{8}$

官

Bucking Coil

Support

Tube
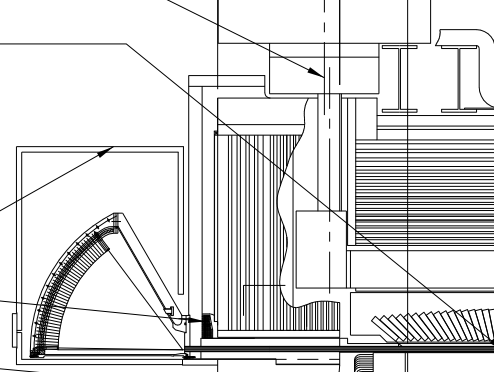

$-\quad-370$
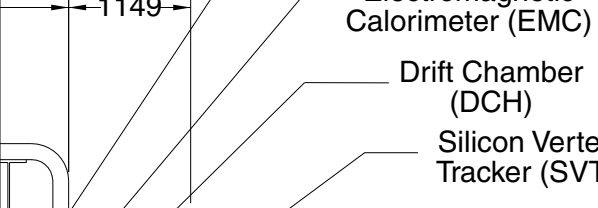

IFR

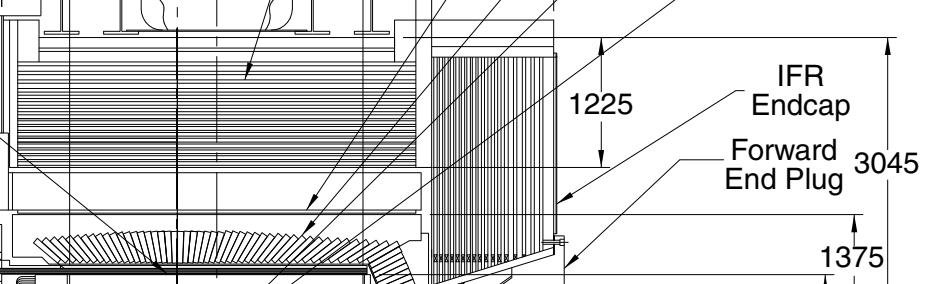

$\mathrm{e}-\cdots$

Q4

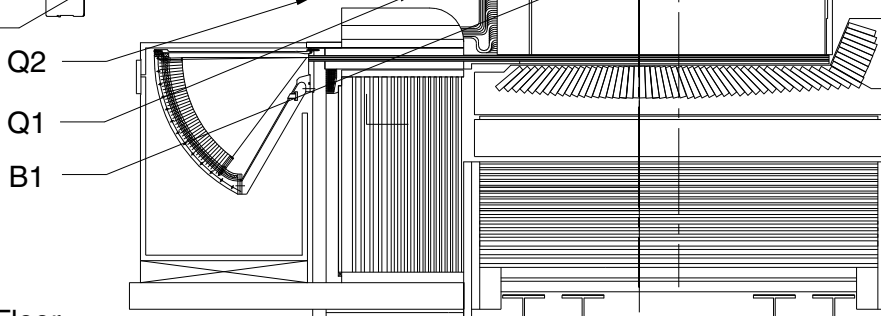

Floor 
- Instrumented Flux Return (IFR): Neutral hadron and $\mu^{ \pm}$identification, hadron rejection.

All detector components but the IFR are embedded in the $1.5 \mathrm{~T}$ superconducting solenoid; the curvature of a charged track in the magnetic field allows determination of the momentum and charge of the particles. Only the tracking and particle identification systems crucial for this analysis are described in the remainder of this chapter. The EMC is detailed in Appendix B.1.

All BABAR detector systems share a common electronics architecture. The frontend electronics (FEE) for any detector component are mounted directly on the detector system; the FEE chain digitizes the detector signals, buffers the digitized output, and forwards that information to the trigger system. Once triggered, the output of an FEE is transferred to storage via readout modules, or ROMs, which connect to the FEE circuits via 1.2 Gbits/s fiber optic cables and provide the standard interface between the detector-specific electronics of the FEE and the fast-control and timing system (FCTS) as well as the event builder. Subsystem-specific feature extraction, in which the relevant features of the raw data (e.g. integrated charge, shape, and timing of digitized waveforms) are extracted, is also done in the ROMs.

\subsection{The Silicon Vertex Tracker}

\subsubsection{Layout and Electronics}

The BABAR SVT has been designed to precisely reconstruct charged particle trajectories and decay vertices near the interaction region. It also provides a measurement of ionization loss $(d E / d x)$ which is supplementary to that provided by the DCH.

The SVT layout is depicted in Figure 2.2. The detector consists of five layers of double-sided silicon strip sensors, organized into three sets of six modules for the inner three layers, and sixteen and eighteen modules for the outer two layers The silicon sensors are double-sided; on one side, the readout strips run parallel to the beam ( $\phi$ strips), while on the other, they run transverse to the beam axis ( $z$ strips). 


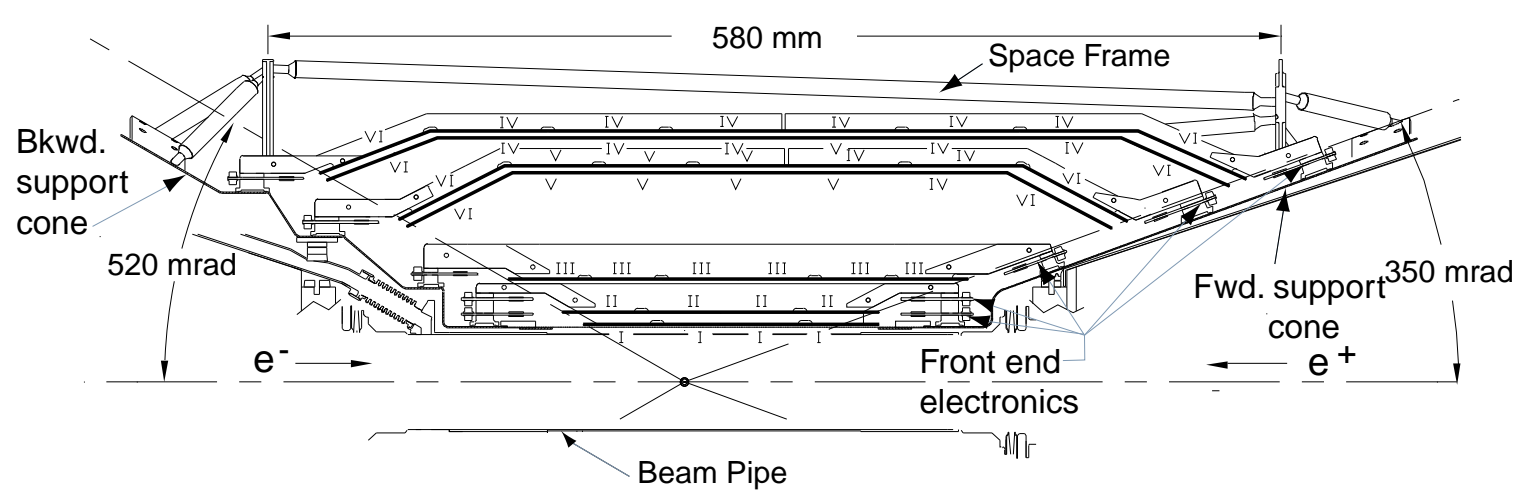

Figure 2.2: Longitudinal cross-section of the SVT.

The readout pitch varies from 50 to $210 \mu \mathrm{m}$; in most cases floating strips (strips that are not read out) lie between two readout strips.

Modules in the inner three layers, which primarily provide position and angle information for measurement of the vertex position, are straight and positioned close to the beam-pipe, in order to minimize the impact of multiple scattering on extrapolation to the vertex. Modules in the fourth and fifth layers are arch-shaped to increase solid angle coverage and the crossing angle for particles near the edges of the module acceptance. The forward acceptance of $350 \mathrm{mrad}$ and the backward acceptance of $520 \mathrm{mrad}$, as well as the $32 \mathrm{~mm}$ radius of the innermost layer relative the interaction point, are determined by the radius of the beam pipe and the size and configuration of the magnets in the interaction region.

Data from the approximately 150,000 channels are delivered via fanout circuits to a custom integrated chip known as the ATOM (A Time-Over-Threshold-Machine). In the ATOM, the signal is processed by a charge-sensitive preamplifier and shaping circuit, and transformed by a programmable-threshold comparator into a pulse whose width is a quasi-logarithmic function of the collected charge. The comparator output is sampled at $15 \mathrm{MHz}$ onto a 193 bin circular buffer. Upon receipt of a Level 1 (L1) trigger (see Section 2.6.1), the time and time-over-threshold of the pulse are retrieved 
from the latency buffer, sparsified, and stored in a four-event buffer; if an L1 Accept is received, the time-over-threshold, the time stamp, and strip address are formatted, serialized, and delivered to the ROM.

\subsubsection{Reconstruction and Performance}

The reconstruction algorithm begins by discarding SVT hits with times more than $200 \mathrm{~ns}$ from the event time as determined by the DCH. The remaining in-time hits are passed on to the cluster finding algorithm, which derives the charge of the individual hits from their time-over-threshold values, and then groups hits from adjacent strips with consistent times into clusters. The cluster position is calculated from the positions of the individual strips, weighted by charge. The clusters are then passed on to both the SVT standalone and combined SVT-DCH tracking algorithms.

Accurate knowledge of both the SVT local and global alignments is critical if the SVT clusters are to be used in precise trajectory measurements. The SVT local

alignment determines the relative positions of the individual SVT modules using primarily tracks from $e^{+} e^{-} \rightarrow \mu^{+} \mu^{-}$events and cosmic rays; these track samples are supplemented with well isolated, high momentum tracks from hadronic events. The SVT global alignment determines the orientation of the SVT as a whole with respect to the DCH coordinate system using a sample of tracks with sufficient numbers of both SVT and DCH hits. The SVT and DCH components of these tracks are fit independently, and the differences between the respective track parameters (as a function of the six global alignment parameters) are minimized.

The local alignment is typically quite stable in time relative to the global SVT positioning. The derivation of the local alignments is complex; as a result, local alignments are performed relatively rarely, typically after magnet quenches or detector access. By contrast, the diurnal movement of the SVT with respect to the DCH requires that the global alignment procedure be performed approximately every 2-3 hours. The achieved spatial resolution for SVT hits, in both $z$ and $\phi$, varies between 20 and $40 \mu \mathrm{m}$ depending on the angle of incidence of the track relative to the SVT module, while the mean $d E / d x$ resolution for minimum-ionizing particles sampled 


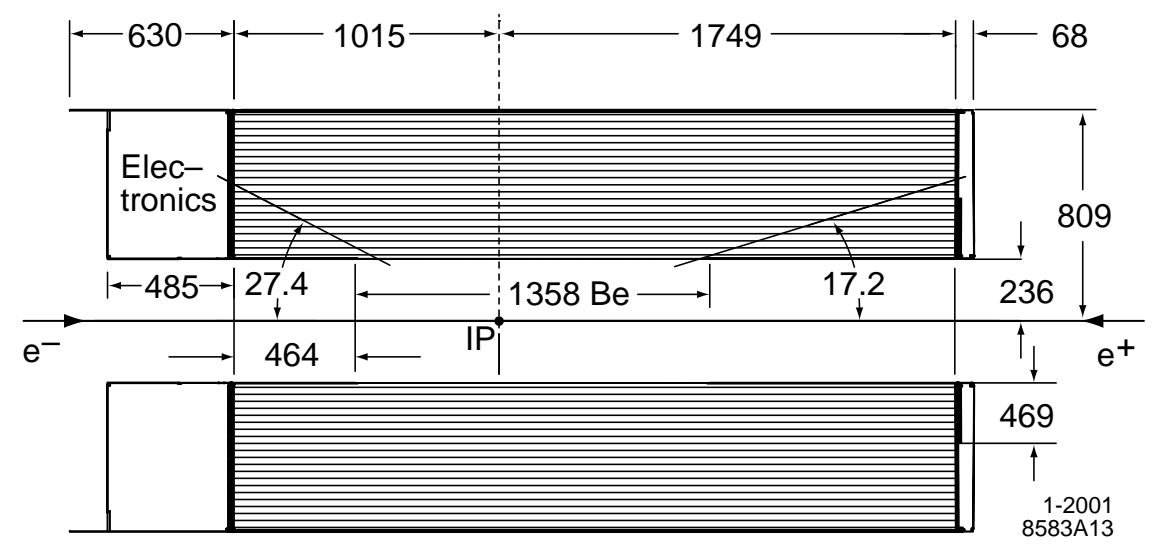

Figure 2.3: Longitudinal cross-section of the DCH.

over five layers is approximately $14 \%$.

\subsection{The Drift Chamber}

The BaBar drift chamber (DCH) is a tracking device which allows for the efficient detection of charged particles and precise measurement of their momenta, as well as the reconstruction of the decay vertices of long-lived particles such as the $K_{s}^{0}$, which may decay outside of the SVT. In addition, the drift chamber measures ionization loss $(d E / d x)$, which provides particle identification information complementary to that provided by the other subsystems. This is particularly critical for low-momentum particles and those in the extreme forward and backward regions of the detector.

\subsubsection{Design and Geometry}

As shown in Figure 2.3, the DCH is enclosed by two concentric cylinders with radii of $236 \mathrm{~mm}$ and $809 \mathrm{~mm}$, approximately 3m in length, and a pair of aluminum endplates. Gold-coated aluminum field wires form 7,104 densely packed hexagonal drift cells, each with a gold-coated tungsten-rhenium sense wires at the center. The cells are arranged in 40 cylindrical layers. Wires in 24 of the 40 layers are strung at small angles (between $\pm 45 \mathrm{mrad}$ and $\pm 76 \mathrm{mrad}$ ) with respect to the z-axis, allowing the extraction of longitudinal as well as axial position information. The layers are 
grouped by fours into ten superlayers; each layer of a superlayer has the same wire orientation (stereo angle) and an equal numbers of cells. Sequential layers within a superlayer are staggered by a half a cell. The stereo angles of the superlayers alternate between the axial (A) and stereo (U and $\mathrm{V})$ pairs, in the order AUVAUVAUVA, as shown in Figure 2.4.

The DCH coverage in azimuth is complete and uniform; the polar acceptance of the $\mathrm{DCH}$ as defined by the most extreme angle at which a particle from the origin crosses at least 20 layers, is $17.2^{\circ}$ in the forward direction and $152.6^{\circ}$ in the backward direction.

The need to minimize multiple scattering, which limits the track resolution below $1 \mathrm{GeV}$, dictates the choice of the physical materials used in the drift chamber construction, as well as the choice of a low-mass gas mixture (an 80:20 helium-isobutane mix). The inner cylindrical wall of the DCH is also kept thin to facilitate matching of SVT and DCH tracks and to minimize the background from photon conversions and interactions; the material in the outer wall and in the forward direction is minimized in order not to degrade the performance of the DIRC and EMC.

\subsubsection{Electronics and Readout}

In order to keep the material in the forward direction to a minimum, the high-voltage (HV) distribution and all DCH readout electronics are mounted at the rear endplate of the chamber. The HV service boards provide the electrostatic potentials for sense, guard, and clearing wires, and pass both signals and ground to the front-end readout electronics. The front-end readout electronics consist of a set of wedge-shaped FrontEnd Assemblies, or FEAs, which plug into connectors on the back side of the HV service boards. Each of the wedge-shaped aluminum boxes of the FEAs contains from two to four amplifier/digitizer (ADB) boards.

The ADB boards themselves hold sets of two 4-channel amplifier integrated circuits(ICs) feeding a single 8-channel digitizer custom ASIC . The custom amplifier IC receives the input signal from the sense wire and produces both a discriminator output signal for the drift time measurement and a shaped analog signal for the 


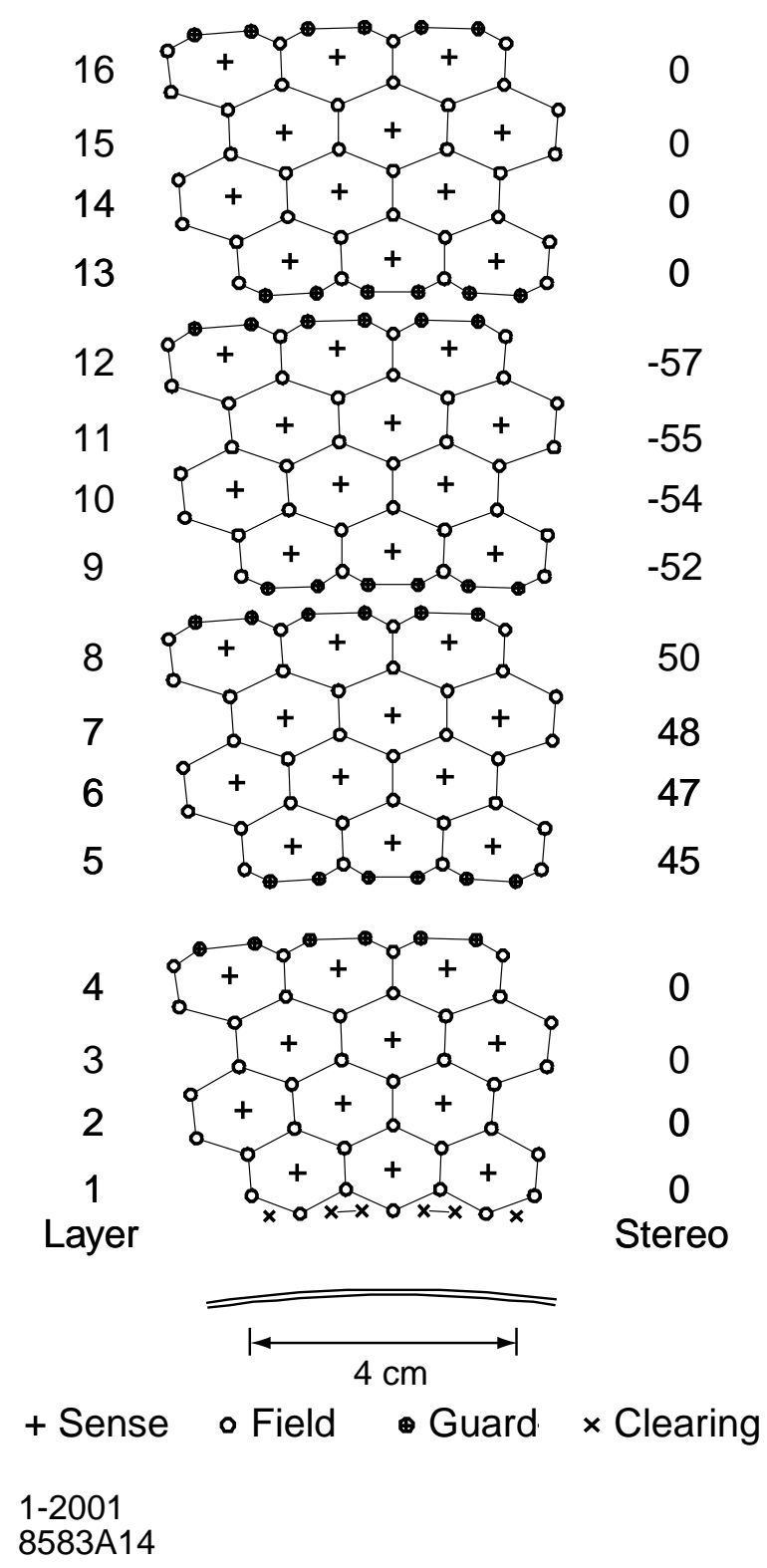

Figure 2.4: Schematic layout of the drift cells in the four inner DCH superlayers. 


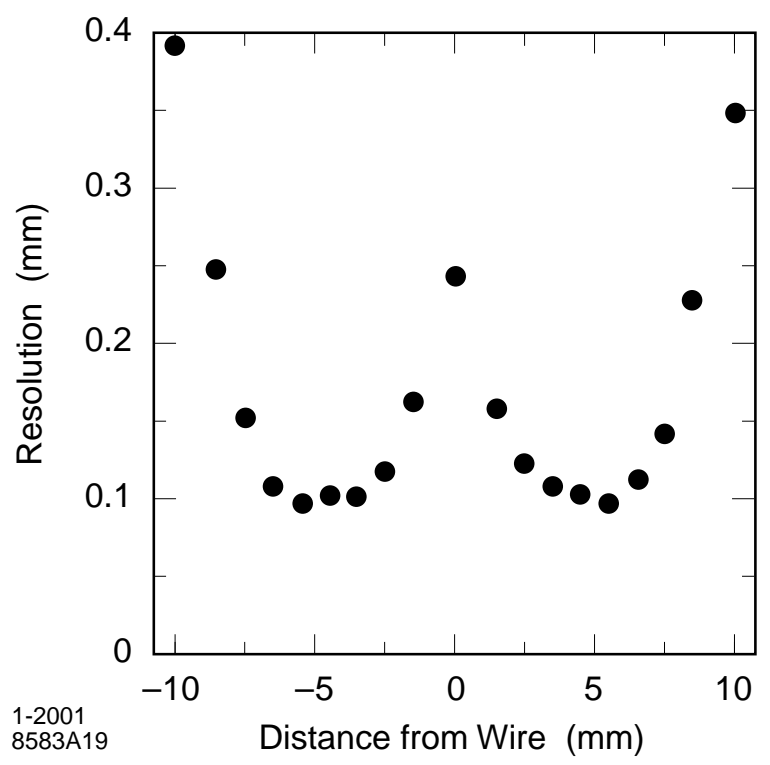

Figure 2.5: DCH single cell resolution.

$d E / d x$ (integrated charge) measurement. The digitizer IC incorporates eight 4-bit Time-to-Digital Converters (TDCs) for time measurement and 6-bit 15MHz FlashAnalog-to-Digital Converters (FADCs) which sample $2.2 \mu$ s of the analog pulse.

Drift chamber-specific feature extraction algorithms convert the raw FADC and TDC information into an ordered set of drift times, the total charge, and a status word. The time and charge are both corrected channel-by-channel for time offsets, pedestals, and gain constants, which are determined by a daily electronics calibration.

\subsubsection{Calibration and Single-Cell Performance}

Knowledge of both the drift time-to-distance relationship and the gas gain are required to determine the drift distance and ionization loss $(d E / d x)$ from the recorded TDC times and accumulated charge. Calibrations for both the time-to-distance relation and $d E / d x$ measurements were developed using cosmic ray data and then implemented for colliding beam data.

The relation between the measured drift time and drift distance is determined using tracks from $e^{+} e^{-}$scattering (Bhabha) and $\mu^{+} \mu^{-}$production. A track trajectory 
is reconstructed using a set of "hits" (TDC times associated with particular drift cells); an estimated drift distance for a cell along the trajectory is determined by computing the distance of closest approach between the track and the signal wire. An average time-to-distance relation is determined for each layer, but separately for the right and left-hand sides of the sense wire, by fitting a sixth-order Chebychev polynomial to a set of estimated drift distances and measured drift times. Figure 2.5 shows the single-cell position resolution as a function of the drift distance for layer 18 of the $\mathrm{DCH}$. The resolution is $100 \mu \mathrm{m}$ away from the boundaries of the cell, but worsens close to the sense wire and the outer cell boundary.

The specific energy loss $\left(\int_{\text {cell }}(d E / d x) d l\right)$ for charged particles traversing the DCH is derived from the measurement of the total charge deposited in each drift cell, as computed by the feature extraction algorithm. The specific energy loss per track is computed as a truncated mean from the lowest 80 percent of the individual cell $d E / d x$ measurements. Corrections are applied to compensate for changes in gas pressure and temperature, differences in cell geometry and charge collection, signal saturation due to space charge buildup, non-linearities in the most probable energy loss at large dip angles, and variation of charge collection as a function of entrance angle. The typical rms resolution, which is limited by the number of samples and Landau fluctuations, is about $7.5 \%$.

\subsubsection{Tracking and Performance}

Reconstruction of charged tracks relies on data from both the SVT and the DCH. Charged tracks are defined by five parameters: $d_{0}$ and $z_{0}$ (transverse distance and $\mathrm{z}$ coordinate at the point of closest approach of the track helix to the $\mathrm{z}$ axis), $\phi_{0}$ (azimuthal angle), $\lambda$ (dip angle with respect to the transverse plane), and $w=1 / p_{t}$ (track curvature). The track reconstruction builds on information from the Level 3 (L3) trigger and tracking algorithms (see Section 2.6.2), first refitting the trigger event time, $t_{0}$, and then performing helix fits to the hits found by the L3 tracking algorithm. A search for additional DCH hits that may belong to a track is performed, and additional track-finding algorithms employed to identify tracks which do not 
traverse the entire $\mathrm{DCH}$ or do not originate from the interaction point.

Tracks found by this algorithm are refit using a Kalman filter, which accounts for local variations in material and magnetic field along the fitting trajectory. They are extrapolated back into the SVT, where SVT track segments are added. Unassociated SVT hits are passed to a pair of standalone SVT track-finding algorithms.

By comparing the number of tracks found in the SVT that extrapolate into the $\mathrm{DCH}$ acceptance to those actually found by the DCH the efficiency for DCH trackfinding has been determined to be $98 \pm 1 \%$. The tracking resolution in the four helix parameters and in transverse momentum $\left(p_{t}\right)$ are determined, in cosmic ray events, to be

$$
\begin{array}{ll}
\sigma_{d_{0}}=23 \mu \mathrm{m} & \sigma_{\phi_{0}}=0.4 \mathrm{mrad} \\
\sigma_{z_{0}}=29 \mu \mathrm{m} & \sigma_{\tan \lambda}=0.53 \cdot 10^{-3}
\end{array}
$$

and

$$
\sigma_{p_{t}} / p_{t}=(0.13 \pm 0.01) \% \cdot p_{t}+(0.45 \pm 0.03) \%
$$

where $p_{t}$ is measured in $\mathrm{GeV} / c$.

\subsection{The Ring-Imaging Cerenkov Detector (DIRC)}

The DIRC (Detector of Internally-Reflected Cherenkov light) is a novel ring-imaging Cherenkov radiation detector used for the identification of charged hadrons. The required momentum coverage of the DIRC is dictated on the one hand by kaon tagging for time-dependent asymmetry measurements, where the typical momentum involved is less than $1 \mathrm{GeV}$, and on the other by $K / \pi$ separation for the $B^{0} \rightarrow$ $\pi^{+} \pi^{-} / K^{+} \pi^{-}$decays, where the relevant momenta lie between 1.7 and $4.2 \mathrm{GeV}$. The minimum transverse momentum required for a charged particle to traverse the $\mathrm{DCH}$ and reach the DIRC is $280 \mathrm{MeV}$, which means there is no need for the DIRC to have any sensitivity below this threshold. 


\subsubsection{Design and Geometry}

The DIRC consists of 144 synthetic fused-silica bars with a refractive index of 1.473, arranged in a 12-sided polygonal barrel around the DCH and an array of 10752 photomultiplier tubes mounted on the stand-off box (SOB) behind the rear IFR doors. The configuration is illustrated in Figure 2.6. Charged particles traversing the bars emit Cherenkov radiation, which propagates by internal reflection to the photomultiplier array in the $\mathrm{SOB}$, allowing reconstruction of the ring and determination of the Cherenkov angle.

The quartz bars are mounted in sets of 12 inside 12 aluminum bar boxes. These bars extend along the entire length of the $\mathrm{DCH}$, covering polar angles down to $25.5^{\circ}$ in the forward direction and $38.6^{\circ}$ in the backward direction, and extend back through the IFR doors to the SOB. The water tank of the SOB flares out from the bars in a conical shape, with twelve sets of $89629 \mathrm{~mm}$ diameter ETL 8125 photomultiplier tubes (PMTs) mounted on the back wall. At the end of each bar is a silica wedge prism, designed to recover photons at wide angles with respect to the bar axis via total reflection. The typical distance between the end of a bar and the photomultipliers is $1.17 \mathrm{~m}$.

The index of refraction of water is 1.346 , close enough to that of the bars to minimize reflection at the interface, and chromatic dispersion is minimized as well. The typical distance between a bar and the PMTs, along with the size of the bars and PMTs themselves, gives a geometric contribution to the single photon Cherenkov angle resolution of $\simeq 7 \mathrm{mrad}$, a contribution somewhat larger than the approximately $5.4 \mathrm{mrad}$ rms spread of the photon production and transmission dispersions. The overall single photon resolution is estimated to be about $10 \mathrm{mrad}$.

\subsubsection{Electronics and Reconstruction}

The DIRC front-end electronics (FEE) are designed to measure the arrival time of each detected Cherenkov photon to an accuracy that is limited by the intrinsic $1.5 \mathrm{~ns}$ transit time spread of the PMTs, and to monitor pulse-height spectra in order to ensure that the PMTs are operating at a voltage which ensures a stable gain (HV 


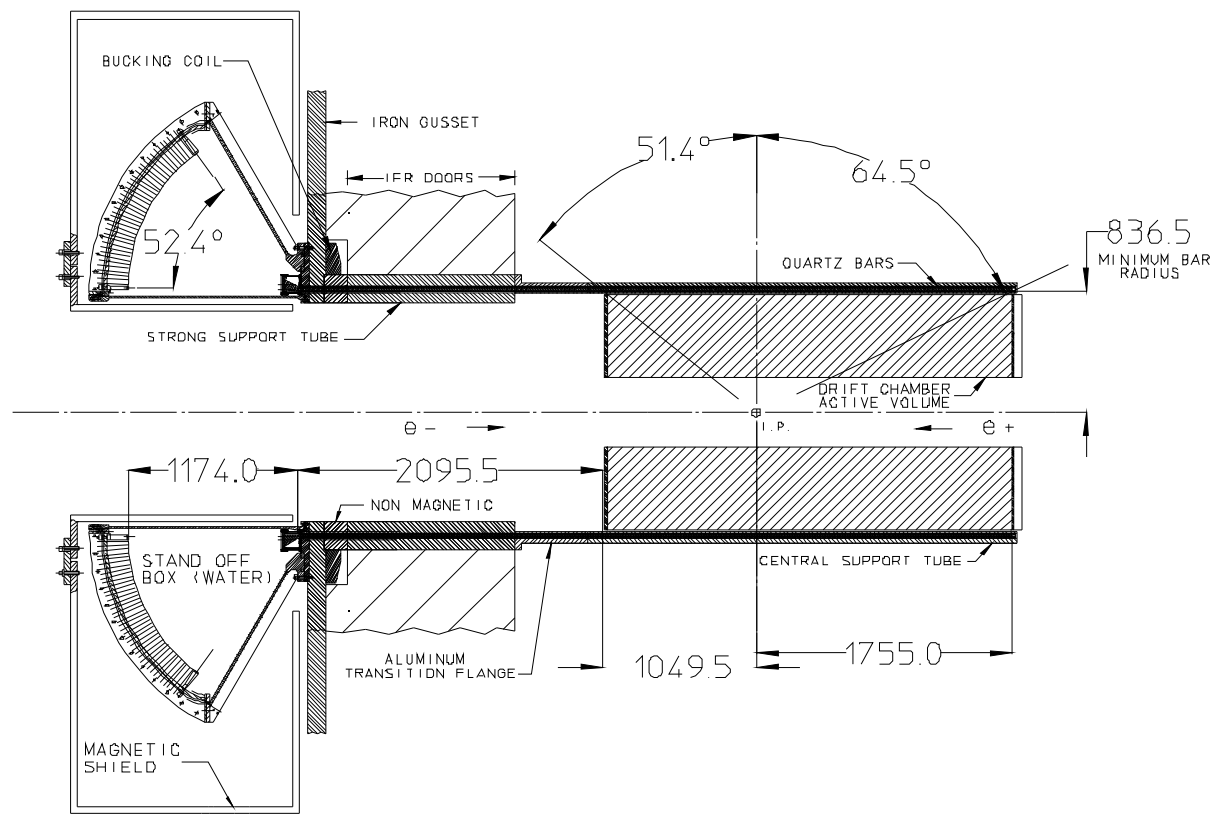

(a)

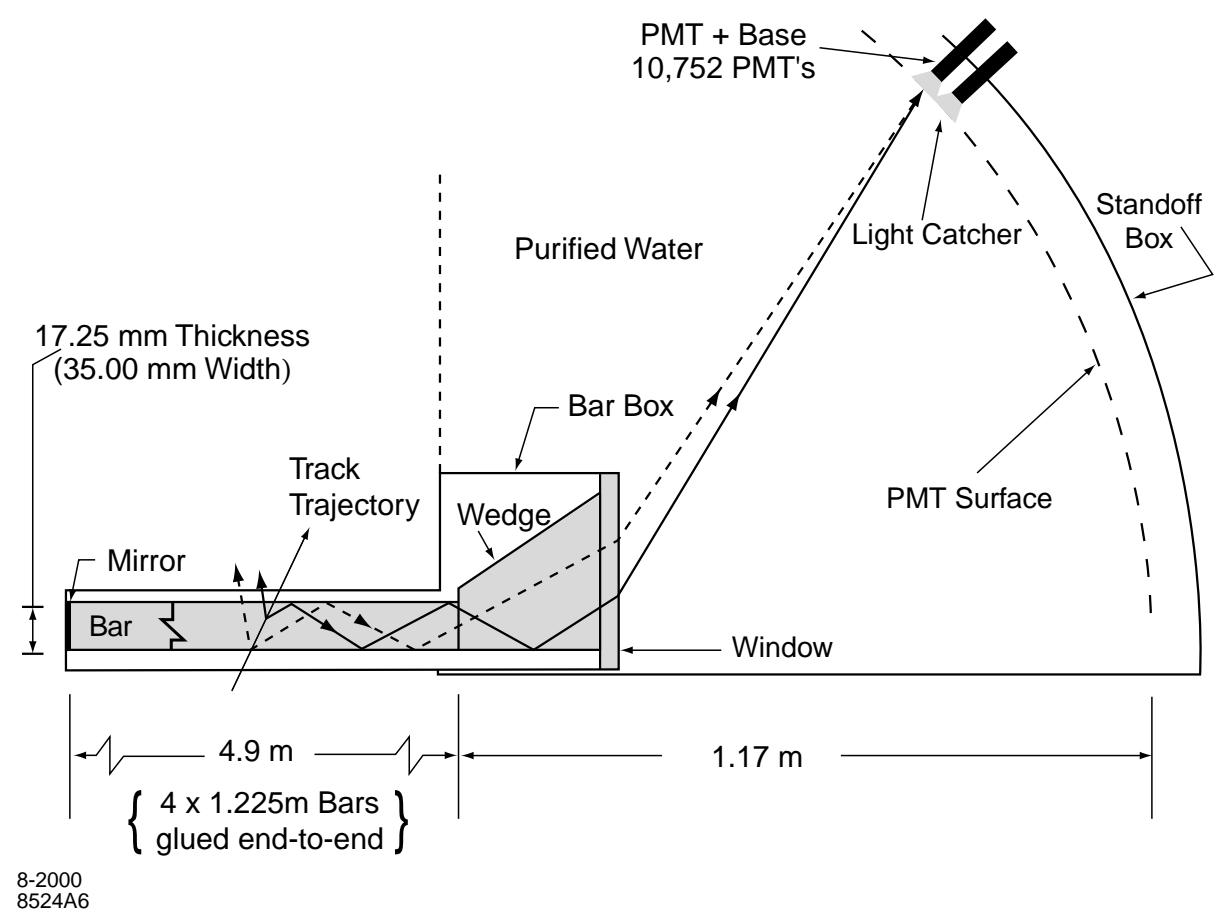

(b)

Figure 2.6: (a) Elevated view of overall DIRC geometry. (b) Bar/SOB transition region. 
plateau) and timing. The DIRC FEE is mounted on the outside of the standoff box, and consists of a set of 168 DIRC Front-end Boards (DFBs), each processing 64 PMT inputs. The four 16-channel custom-IC TDCs allow for an independent timing measurement for each TDC, while the single 8-bit flash ADC (FADC) multiplexes the pulse-height information for all 64 channels. The TDC has $0.5 \mathrm{~ns}$ binning, allowing the photon arrival time to be determined to better than the intrinsic $1.5 \mathrm{~ns}$ accuracy. The digitized information is shipped from the FEE to the ROMs via optical fibers.

Calibration of the DIRC TDCs is achieved using 1 ns pulses from blue LED light pulsers; the LEDs, are pulsed at roughly $2 \mathrm{kHz}$. Adjacent sectors are pulsed in a staggered fashion to prevent light crosstalk. Approximately 65,000 pulses per PMT are used in the calibration, to achieve a statistical accuracy of less than 0.1 ns. A complementary method compares observed and expected light arrival times associated with tracks in actual collision data; this method yields an improved resolution (about $15 \%$ better) and consistent results.

Reconstruction of the emission angle and the arrival time of the Cherenkov photons produced by a charged track in the DIRC is done using observed space-time coordinates of the PMT signals, transformed into the Cherenkov coordinate system $\left(\theta_{c}\right.$ and $\phi_{c}$, the polar and azimuthal angles relative to the cone direction, and $\delta t$, the difference between the observed and expected arrival times). A set of threedimensional vectors, from the end of a radiator bar to the center of each coupled PMT, are extrapolated into the radiator bar using Snell's law and determined up to a 16-fold ambiguity. The uncertainties derive from the last reflection in the bar (forward/backward, left/right), whether the photon scattered off of the coupling wedge, and whether the photon initially propagated forward or backward. The timing resolution cannot provide competitive position information, but is used to suppress beaminduced background by about a factor of 40, to exclude other tracks in the same event, and to reduce the 16-fold ambiguity to a three-fold ambiguity. The reconstruction algorithm then maximizes the likelihood of the entire event, based on the individual track likelihoods for the electron, muon, pion, kaon, and proton hypotheses. When coupled with $d E / d x$ information from the SVT and DCH, the DIRC achieves better than $90 \%$ kaon identification efficiency, with a less than $3 \%$ pion misidentification 
rate, for tracks which intersect with the radiator bars and have momenta between 0.5 and $3 \mathrm{GeV}$.

\subsection{Trigger}

PEP-II operating luminosities exceed the original design luminosity; at the design luminosity, beam-related background events occur at rates greater than $20 \mathrm{kHz}$, while useful events ( $B \bar{B} \tau^{+} \tau^{-}$, etc.) occur at rates of only a few Hz. The role of the trigger system is to select events of interest while rejecting the rest, thus reducing the total data-taking rate to level manageable for online reconstruction (less than $120 \mathrm{~Hz}$ ). The trigger must operate with a high efficiency (at least $99 \%$ for $B \bar{B}$ events) and a low deadtime (no more than $1 \%$ ).

The trigger is implemented as a two-tier system: Level 1 (L1), implemented in hardware, is designed to reduce the input rate to Level 3 to $1 \mathrm{kHz}$, while Level 3 (L3) is implemented in software. For historical reasons, there is no Level 2 trigger.

\subsubsection{The Level 1 Trigger}

The Level 1 (L1) trigger consists of three hardware components, each based on information from a specific subsystem: the DCT, based on DCH information, the EMT, based on EMC information, and the IFT, based on IFR information (IFT) and used primarily for diagnostic purposes.

\section{The Drift Chamber Trigger}

The DCT takes data from the DCH cells and implements a fast 24 module Track Segment Finder (TSF) using the $\phi$ coordinate and the drift times of the DCH hits. The segments are passed to the Binary Link Tracker (BLT), which bins the segments into supercells dividing the DCH into $32 \phi$ bins and 10 superlayers. The BLT links segments in contiguous supercells, starting from the innermost drift-chamber layer. Eight transverse momentum discriminator modules (PTD) determine the number of tracks above a certain threshold. The output of the DCT is a set of 16 bit trigger 
primitives which categorize the BLT and PTD results into short tracks (traversing half the DCH), long tracks (traversing the entire DCH), and high $p_{t}(>800 \mathrm{MeV})$ tracks.

\section{The Electromagnetic Calorimeter Trigger}

For trigger purposes, the EMC is divided into 280 towers, $7 \times 40(\theta \times \phi)$. For each tower, all crystal energies above a $20 \mathrm{MeV}$ threshold are summed and sent to the EMT every 269 ns. The towers are also grouped into the $40 \phi$-sectors. The patterns of energy deposition and arrival time in the $\phi$-sectors are used to form a set of trigger primitives.

The DCT and EMT inputs are processed by a global trigger (GLT) to form specific L1 triggers. Processing times for the DCT and EMT both are about $5 \mu$ s with an additional $4 \mu \mathrm{s}$ for the GLT to process and initiate readout by the ROMs. The combined L1 triggers achieve nearly $100 \%$ efficiency for generic $B \bar{B}$ events.

\subsubsection{The Level 3 Trigger}

The Level 3 trigger (L3) involves a basic reconstruction of the event in both the DCH and EMC. As such it consists of a track finding algorithm for the DCH and a clustering algorithm for the EMC. The kinematics and topology of the reconstructed event, as given by the results of these two algorithms, allows the event to be categorized for acceptance or rejection.

The track-finding algorithm uses a Monte Carlo-derived lookup table of hit patterns in order to join track segments from the TSF to form a track. If a pattern of segment hits matches an entry in the lookup table, the reconstructed track is refined by an iterative fitting algorithm, which adds or drops hits based on their proximity to the fitted trajectory. The L3 clustering algorithm forms clusters from adjacent energy depositions that are within $1.3 \mu$ s of the event time, and have more than 20 $\mathrm{MeV}$ of energy. For clusters with at least $100 \mathrm{MeV}$ of energy, the centroid, lateral energy profile, and average cluster time are calculated. 
Table 2.1: Generic Monte Carlo samples used in the analysis.

\begin{tabular}{||c|c|c|c||}
\hline Event Type & Number of Events (millions) & cross-section (nb) & $L\left(f b^{-1}\right)$ \\
\hline Generic $B^{+} B^{-}$ & 590 & 0.525 & 1123 \\
Generic $B^{0} \bar{B}^{0}$ & 581 & 0.525 & 1106 \\
Generic $c \bar{c}$ & 472 & 1.3 & 363 \\
Generic $u \bar{u}+d \bar{d}+s \bar{s}$ & 706 & 2.09 & 338 \\
\hline
\end{tabular}

\section{Performance}

L3 information allows QED processes useful for calibration purposes, such as (radiative) Bhabha scattering or $e^{+} e^{-} \rightarrow 2 \gamma$ events, to be identified and passed at reduced rates, while multiplicity criteria identify hadronic events from both $B \bar{B}$ decays and the continuum. The combined L1 and L3 trigger achieves better than $99.9 \%$ efficiency for $B \bar{B}$ events and better than $95 \%$ efficiency for continuum events.

\subsection{The Data}

We use data collected on the $\Upsilon(4 \mathrm{~S})$ resonance with the BABAR detector at PEPII , corresponding to an integrated luminosity of $210 \mathrm{fb}^{-1}$. An additional $21 \mathrm{fb}^{-1}$ of data, collected $40 \mathrm{MeV}$ below the resonance peak (referred to as off-resonance data), is used to study the background from light-quark and $c \bar{c}$ production. Within the collision environment, the $\Upsilon(4 \mathrm{~S})$ production which actually produces $B \bar{B}$ pairs accounts for about a quarter of the total cross-section of hadronic production; the rest is continuum production. Other processes such as $e^{+} e^{-} \rightarrow \mu^{+} \mu^{-}$and $e^{+} e^{-} \rightarrow \tau^{+} \tau^{-}$ are produced at rates comparable to the $\Upsilon(4 \mathrm{~S})$ production while Bhabha scattering occurs at rates nearly forty times higher. The total number of $B \bar{B}$ pairs produced in the data is determined by comparing the yield of hadronic events in the on- and off-peak data [137]. The yield in the off-resonance data is scaled by the luminosity determined from the yield of $e^{+} e^{-} \rightarrow \mu^{+} \mu^{-}$to the on-resonance data and subtracted. The number of $B \bar{B}$ pairs is determined to be 232 million assuming equal production 
of $B^{0} \bar{B}^{0}$ and $B^{+} B^{-}$and neglecting other decay modes of $\Upsilon(4 \mathrm{~S})^{2}$.

The Monte Carlo simulations of specific channels as well as generic continuum and $B \bar{B}$ processes is based on a detailed detector simulation using GEANT4 [138]. Background estimates from these samples are obtained by scaling the event yields using the equivalent luminosity for the simulated sample based on the known cross-section for these processes [139]. The corresponding luminosities of the generic background Monte Carlo (MC) samples used in the analysis are summarized in Table 2.1.

\footnotetext{
${ }^{2}$ The other decay modes of $\Upsilon(4 \mathrm{~S})$ constitute $<4 \%$ at $95 \%$ confidence level (CL) [20]
} 


\section{Chapter 3}

\section{$B^{+} \rightarrow p \bar{p} K^{+}$Branching Fraction Measurement}

This chapter describes a measurement of the baryonic three-body decay final state $B^{+} \rightarrow p \bar{p} K^{+}$. Charge-conjugate reactions are implicitly included throughout this document. An earlier measurement [131] of the branching fraction for this channel gave $\mathcal{B}\left(B^{+} \rightarrow p \bar{p} K^{+}\right)=\left(5.7_{-0.6}^{+0.7} \pm 0.7\right) \times 10^{-6}$, after removing the charmonium regions $2.85<m_{p \bar{p}}<3.128 \mathrm{GeV} / c^{2}$ and $3.315<m_{p \bar{p}}<3.735 \mathrm{GeV} / c^{2}$. This channel is particularly interesting for the possible presence of exotic [45], [117] intermediate states. We also isolate the decays $B^{+} \rightarrow X_{c \bar{c}} K^{+}$, where $X_{c \bar{c}}=\eta_{c}$ and $J / \psi$ decaying to $p \bar{p}$, measure the width of the $\eta_{c}$, and search for the decay $B \rightarrow p \bar{\Lambda}(1520)$.

\subsection{Track reconstruction requirements}

The "prompt" tracks which are produced at the $B$ decay vertex are required to

- Contain at least 12 DCH hits.

- Originate from the interaction region point within $10 \mathrm{~cm}$ along the beam direction and $1.5 \mathrm{~cm}$ in the transverse plane.

- Have minimum transverse momentum: $p_{t}>0.1 \mathrm{GeV} / \mathrm{c}$. 
- Have non-zero $B$-vertex fit $\chi^{2}$ probability.

\subsection{Particle Identification (PID)}

Particle identification used in this analysis is described in details in Ref. [140]. The charged particles are identified using corresponding likelihood ratios formed from $d E / d x$ information from the DCH and SVT for $p_{t}<0.7 \mathrm{GeV} / \mathrm{c}$, the measured Cherenkov angle, and the number of photons observed in the DIRC for $p_{t}>0.6 \mathrm{GeV} / \mathrm{c}$.

The likelihood selector calculates a product of likelihoods for each particle hypothesis: $\mathcal{L}_{\text {ipart }}=\mathcal{L}_{\text {ipart }}^{D I R C} \times \mathcal{L}_{\text {ipart }}^{D C H} \times \mathcal{L}_{\text {ipart }}^{\text {DVT }}$. The DCH and SVT Likelihoods are already calculated by comparing the measured $d E / d x$ against the expected $d E / d x$ from the Bethe-Bloch parametrization for each particle hypothesis:

$$
\mathcal{L}^{D C H, S V T}=\frac{d E / d x(\text { measured })-d E / d x(\text { Bethe }- \text { Bloch })}{\sigma},
$$

where $\sigma$ is an error on the measured $d E / d x$ value. The DCH likelihood is calculated based on a Gaussian Probability Distribution Function (PDF), and the SVT likelihood is calculated based on a Bifurcated Gaussian PDF.

The DIRC likelihood cannot be "calculated" in this way, since there are significant tails in the distributions of the fit Cherenkov angle and the number of photons. To minimize the effect of these tails, a binned likelihood is constructed from the Cherenkov angle (DIRC angle), number of photons $\left(N_{\gamma}\right)$, and track quality (Trkqual). This new likelihood is stored in a lookup table binned in

$$
\begin{aligned}
& \text { ("Momentum in the laboratory frame" }-100 \mathrm{MeV} / \mathrm{c} \text { bins }) \\
& \quad \times(\text { "DIRC angle" }-3 \text { bins }) \\
& \times\left(\text { "N } \mathrm{N}_{\gamma}, \text { Trkqual" }-4 \text { bins }\right) .
\end{aligned}
$$

Only three "DIRC angle" bins, corresponding to the pion, kaon and proton bands are used. The four " $N_{\gamma}$,Trkqual" bins are formed using the Poisson probability for the number of photons observed, the layer of the last DCH hits, and the calorimeter 
energy deposit. This " $N_{\gamma}$, Trkqual" binning is an attempt to identify problem tracks for the DIRC reconstruction. The lookup tables are filled using MC truth-matched tracks $^{1}$. The value of likelihood is the probability that a MC truth-matched track has the measured quantities of that bin. This binned DIRC likelihood is designed to treat the tails in the DIRC response, especially near particle thresholds. It does not separate the DIRC angle bands well at higher momentum, so this binned likelihood is multiplied by a Gaussian DIRC angle likelihood for momentum $>1.5 \mathrm{GeV} / c$.

Finally, the charged particle is assigned to a particular category (electron, muon, pion, kaon or proton) by requiring different cuts on the relevant likelihood ratios. The following cuts are used in this analysis:

- Proton and anti-proton candidates are required to have likelihood ratios $L_{p} / L_{K}>$ 1.3 and $L_{p} / L_{\pi}>0.5$. This yields a proton efficiency of $93 \%$ with $9 \%$ kaon misidentification rate.

- Kaon candidates are required to have likelihood ratios $L_{K} / L_{\pi}>0.8176$ and $L_{K} / L_{p}>0.018$, and for $p_{t}>0.40 \mathrm{GeV} / c$ not passing the electron criteria. The resulting kaon efficiency is $87 \%$ with $2 \%$ pion mis-identification probability.

\section{$3.3 \quad B^{+} \rightarrow p \bar{p} K^{+}$Selection}

The $B$ candidate is formed by combining the momentum four-vectors of the proton, the anti-proton and the kaon candidates. Two kinematic variables are used to isolate the $B \rightarrow p \bar{p} K^{+}$signal taking advantage of the kinematic constraints of $B$ mesons produced at the $\Upsilon(4 S)$. The first is the beam-energy-substituted mass,

$$
m_{E S}=\left[\left(E_{C M}^{2} / 2+\mathbf{p}_{i} \cdot \mathbf{p}_{B}\right)^{2} / E_{i}^{2}-\mathbf{p}_{B}^{2}\right]^{1 / 2},
$$

where $E_{C M}$ is the total center-of-mass energy of the $e^{+} e^{-}$collision. Here, the fourmomentum of the initial $e^{+} e^{-}$system is $\left(E_{i}, \mathbf{p}_{i}\right)$ and $\mathbf{p}_{B}$ is the momentum of the

\footnotetext{
${ }^{1}$ Truth-matching is an algorithm used to associate a reconstructed track in the Monte Carlo simulation to its true origin.
} 

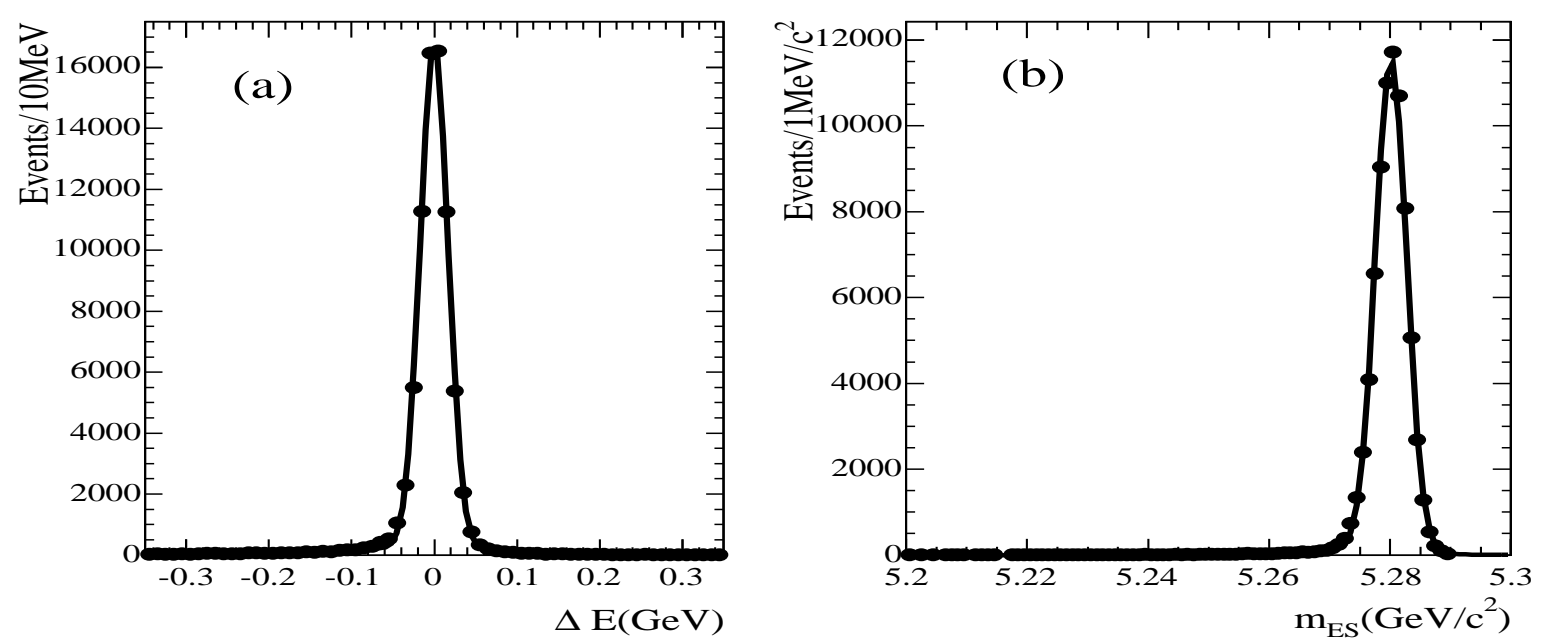

Figure 3.1: (a) $\Delta E$ distribution and (b) $m_{E S}$ distribution of truth-matched $B^{+} \rightarrow$ $p \bar{p} K^{+}$signal Monte Carlo events.
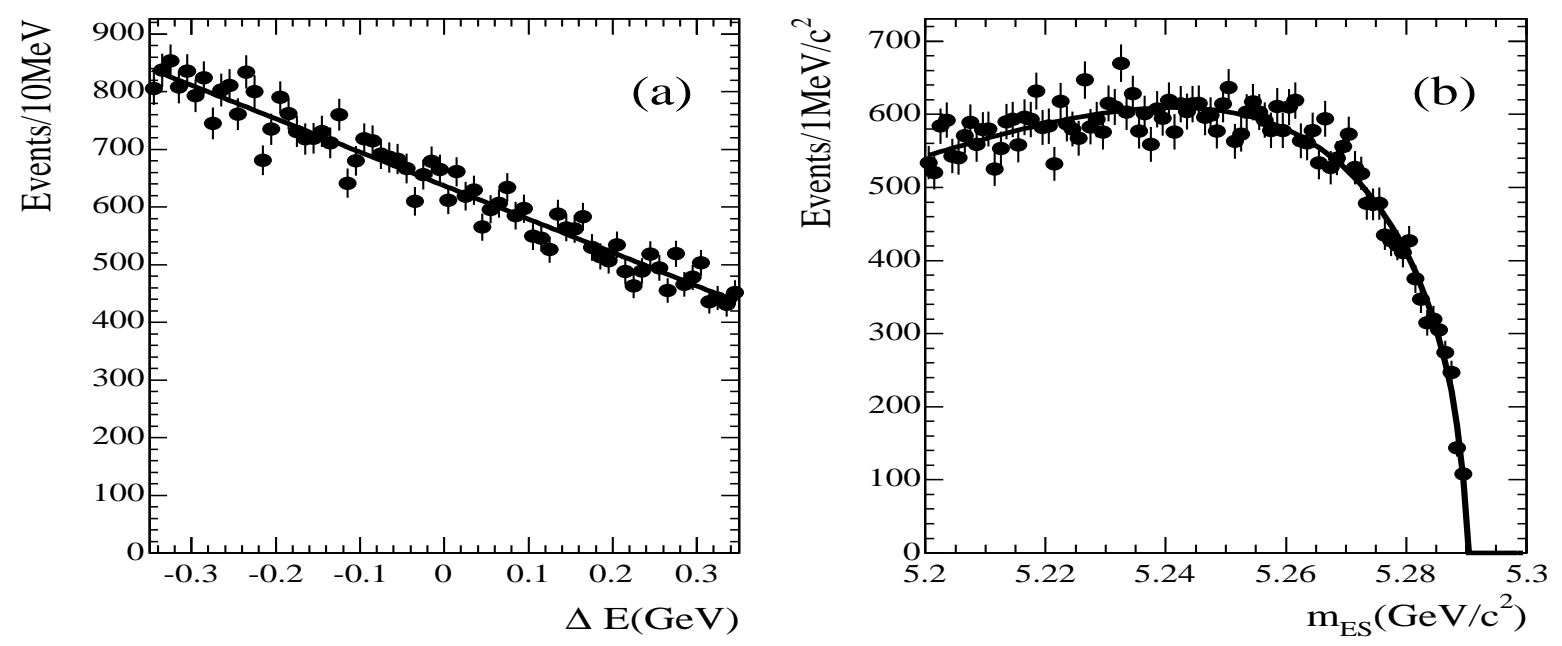

Figure 3.2: (a) $\Delta E$ distribution and (b) $m_{E S}$ distribution of off-peak data background events reconstructed in $B^{+} \rightarrow p \bar{p} K^{+}$mode. 
reconstructed $B$ candidate, both measured in the laboratory frame. The second variable is

$$
\Delta E=E_{B}^{*}-E_{C M} / 2
$$

where $E_{B}^{*}$ is the $B$-candidate energy in the center-of-mass frame. Distributions of $\Delta E$ and $m_{\mathrm{ES}}$ for the $B^{+} \rightarrow p \bar{p} K^{+}$Monte Carlo events are shown in Figure 3.1 and are fit with double Gaussian distributions. $\Delta E$ and $m_{E S}$ have resolutions of approximately $17 \mathrm{MeV}$ and $2.6 \mathrm{MeV} / \mathrm{c}^{2}$, respectively for the $p \bar{p} K^{+}$signal. The corresponding distributions for the background are shown in Figure 3.2 and are fit with a linear function for the $\Delta E$ distribution and an ARGUS function [141] for the $m_{\mathrm{ES}}$ distribution. The initial selection requires $|\Delta E|<0.35 \mathrm{GeV}$ and $m_{E S}>5.2 \mathrm{GeV}$.

\subsection{Background Characterization}

Expected background contributions are studied with generic Monte Carlo samples described in Section 2.7. There are two main sources of backgrounds: continuum background $\left(e^{+} e^{-} \rightarrow q \bar{q}\right.$, where $\left.q=u, d, s, c\right)$, and $B$ background where other $B$ meson-decay modes result in the $p \bar{p} K^{+}$combinations.

\subsubsection{Continuum $(q \bar{q})$ Background}

Continuum events are rich in baryons, producing protons and anti-protons either as primary decay products or as the result of a heavier baryon parent decaying into them (e.g. $\Delta, \Lambda, \Sigma, \Lambda_{c}, \Sigma_{c}, \Xi_{c}$ ). Several topological variables provide discrimination between the large continuum background $\left(e^{+} e^{-} \rightarrow q \bar{q}\right.$, where $\left.q=u, d, s, c\right)$, which tends to be collimated along the original quark direction, and the more spherical $B \bar{B}$ events.

Four variables shown in Figure 3.3 are employed to exploit the difference in event topology.

- The thrust angle $\theta_{t h r}^{B}$ is the angular difference between the thrust axis of the $B$-candidate and the $z$-axis. The thrust axis is defined as the direction in the $\Upsilon(4 S)$ rest frame along which the sum of longitudinal momenta of charged and 

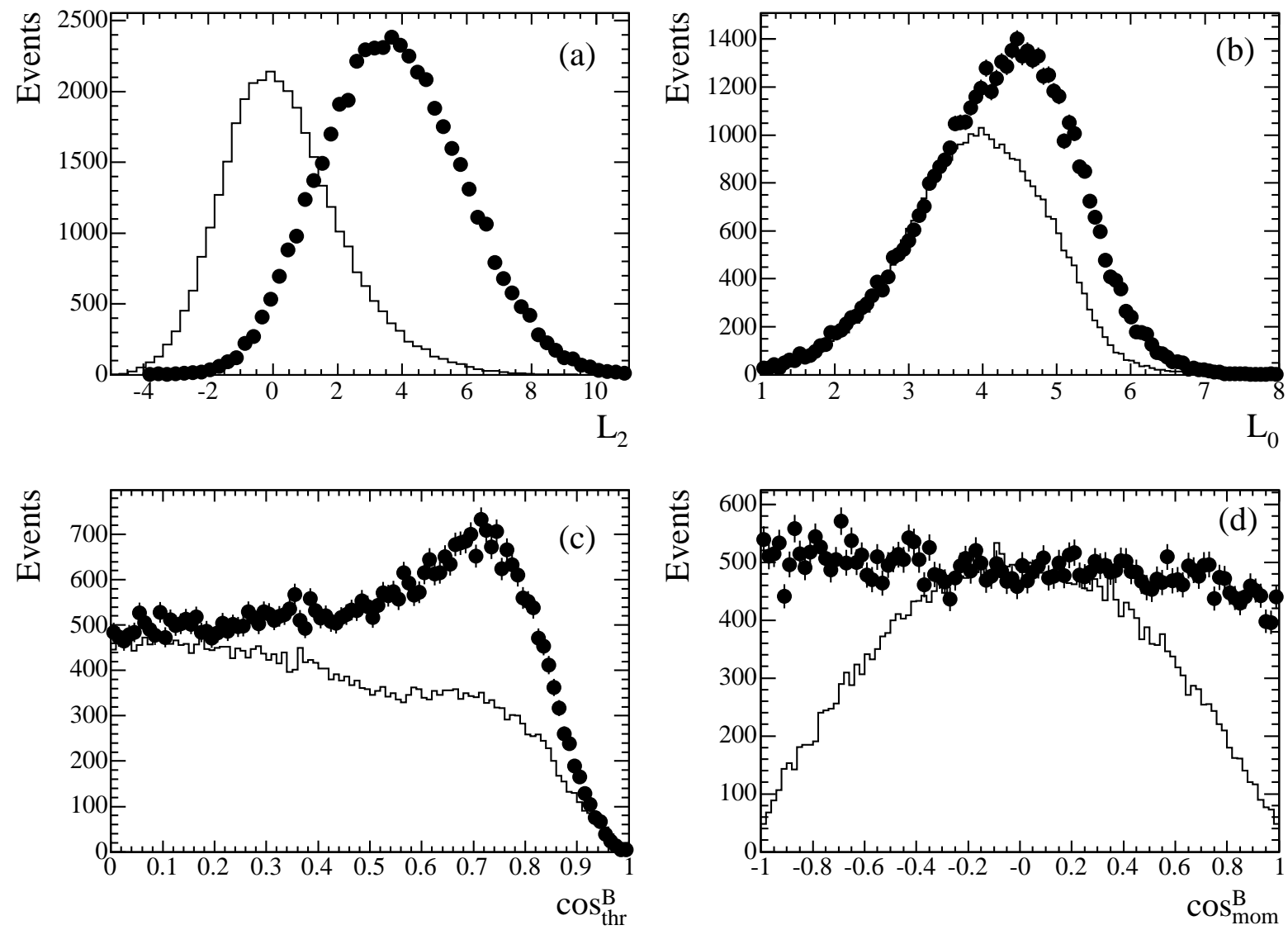

Figure 3.3: $B^{+} \rightarrow p \bar{p} K^{+}$signal Monte Carlo (histogram) - off-peak data background (dots) comparisons for: (a) $L_{2}$, (b) $L_{0}$, (c) $\cos \theta_{t h r}^{B}$, and (d) $\cos \theta_{\text {mom }}^{B}$. Normalizations in the plots are random. 
neutral particles is maximized. The cosine of this angle, $\cos \theta_{t h r}^{B}$ is uniformly distributed for signal events. Because various decay particles in continuum events have highly correlated momentum directions along the jet axis, $\cos \theta_{t h r}^{B}$ peaks strongly at \pm 1 (without the acceptance effects).

- The angle between the $B$-candidate direction and the $z$-axis in the $\Upsilon(4 S)$ rest frame, $\theta_{m o m}^{B}$, is also useful for discrimination. The distribution of $\cos \theta_{m o m}^{B}$ is flat for continuum events. For signal events (without the acceptance cuts) the distribution takes a $1-\cos ^{2} \theta_{\text {mom }}^{B}$ shape.

- Momentum-weighted Legendre polynomials can be used to quantify the sphericity of events, defined as $L_{i}=\sum_{j} p_{j} \mathcal{L}_{i}\left(\theta_{j}\right)$; where the sum is over all charged and neutral particles in the other (non-candidate) $B$ meson, $\theta_{j}$ is the angle between the $B$-candidate thrust axis and the $j$ th particle, and $\mathcal{L}_{i}$ is the $i$ th Legendre polynomial. This analysis uses zeroth- and second-order Legendre polynomial momentum moments, $L_{0}=\sum_{i}\left|p_{i}^{*}\right|$ and $L_{2}=\sum_{i}\left|p_{i}^{*}\right|\left[\left(3 \cos ^{2} \theta_{t h r_{B, i}}-1\right) / 2\right]$, where $p_{i}^{*}$ are the center-of-mass momenta for the tracks and neutral clusters that are not associated with the $B$ candidate.

These variables are correlated and thus cannot be used in a maximum likelihood fit as separate observables. To avoid this problem a linear combination of these variables, known as a Fisher discriminant, $\mathcal{F}=\sum_{i=1 . .4} \lambda_{i} \Lambda_{i}[142]$, is used in this analysis. The $\Lambda_{i}$ are the event shape variables: $L_{0}, L_{2},\left|\cos \theta_{t h r}^{B}\right|$ and $\cos \theta_{m o m}^{B}$. The coefficients $\lambda_{i}$ are determined by maximizing the separation between the means of the resulting signal and background distributions in terms of standard deviations [143]:

$$
\lambda_{i}=\sum_{j=1}^{N}\left(\left(\sigma_{i j}^{S G}\right)^{2}+\left(\sigma_{i j}^{B G}\right)^{2}\right)^{-1}\left(\overline{\Lambda_{i}^{S G}}-\overline{\Lambda_{i}^{B G}}\right)
$$

where the signal distribution is obtained from $B^{+} \rightarrow p \bar{p} K^{+}$simulated events that are distributed uniformly in phase-space $\left(B^{+} \rightarrow p \bar{p} K^{+}\right.$"signal" Monte Carlo) and the background shape comes from off-peak data ${ }^{2}$. The Fisher Discriminant for the signal

\footnotetext{
${ }^{2}$ Off-peak data consists of continuum events only, as it is taken at $E_{C M}<2 M_{B}$.
} 

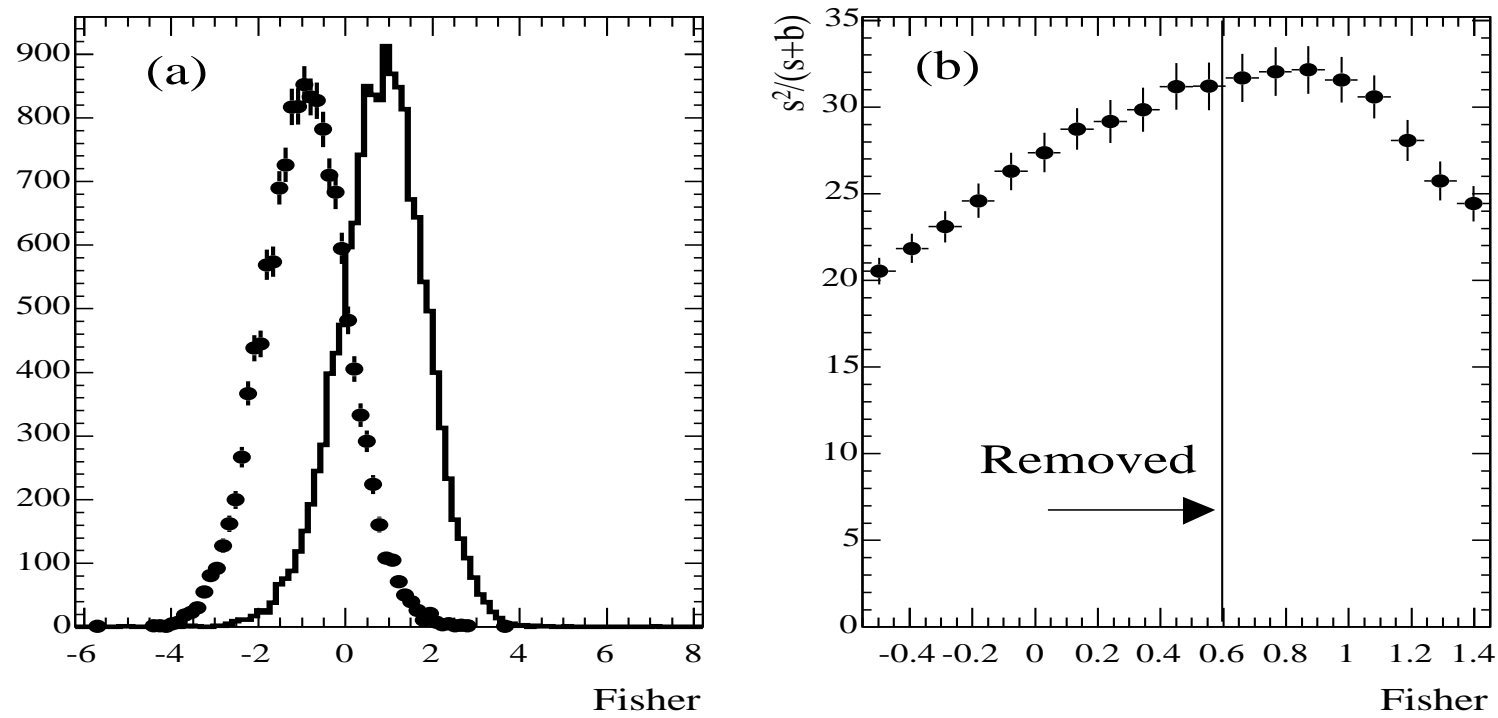

Figure 3.4: (a) Fisher discriminant in signal Monte Carlo (histogram) and off-peak data (dots); (b) Fisher discriminant cut optimization.

Monte Carlo and for the off-peak data is shown in Figure 3.4(a).

\subsubsection{B Background}

The main source of the $B \bar{B}$ backgrounds is the $b \rightarrow c \bar{c} s$ transitions that subsequently decay into the same final states as $B^{+} \rightarrow p \bar{p} K^{+}$signal events. In particular, these include $B^{+} \rightarrow X_{c \bar{c}} K^{+}, X_{c \bar{c}} \rightarrow p \bar{p}$ and $X_{c \bar{c}}=\eta_{c}, J / \psi, \psi(2 S), \chi_{c 0,1,2}$ (the so-called "charmonium background"). These channels have branching fractions compatible to those of the signal, as shown in Table 3.1. To check for additional $B \bar{B}$ backgrounds that might mimic the $B^{+} \rightarrow p \bar{p} K^{+}$signal, we study generic $B \bar{B}$ Monte Carlo as well as a set of samples of exclusive $B$ decay simulated events for potential "charmoniumless" backgrounds. These $b \bar{b}$ background events are dominated by events where some tracks are either mis-identified with $p / \bar{p}$ being mostly pions from $\rho^{0}, D^{0}$ and $B$ decays and $K^{ \pm}$being mostly kaons from $D^{0}$ decays. The expected $B \bar{B}$ "charmoniumless" background contribution is small and indistinguishable from continuum backgrounds. 
Table 3.1: The expected rates for the events of the type $B^{+} \rightarrow X K^{+}, X \rightarrow p \bar{p}[20]$.

\begin{tabular}{||c|c|c|c|c||}
\hline \hline$X$ & Mass $_{X}^{P D G}$ & $B F_{X \rightarrow p \bar{p}}$ & $B F_{B \rightarrow X K^{+}}$ & $B F_{B \rightarrow X(p \bar{p}) K^{+}}$ \\
\hline$\eta_{c}$ & $2979.7 \pm 1.5$ & $(1.3 \pm 0.4) \cdot 10^{-3}$ & $(9.0 \pm 2.7) \cdot 10^{-4}$ & $(1.08 \pm 0.48) \cdot 10^{-6}$ \\
\hline$J / \psi$ & $3096.87 \pm 0.04$ & $(2.12 \pm 0.10) \cdot 10^{-3}$ & $(1.00 \pm 0.04) \cdot 10^{-3}$ & $(2.12 \pm 0.13) \cdot 10^{-6}$ \\
\hline$\chi_{c 0}(1 P)$ & $3415.1 \pm 0.8$ & $(2.2 \pm 0.3) \cdot 10^{-4}$ & $\left(6.0_{-2.1}^{+2.4}\right) \cdot 10^{-4}$ & $\left(1.32_{-0.51}^{+0.61}\right) \cdot 10^{-7}$ \\
\hline$\chi_{c 1}(1 P)$ & $3510.51 \pm 0.12$ & $(7.2 \pm 1.3) \cdot 10^{-5}$ & $(6.8 \pm 1.2) \cdot 10^{-4}$ & $(4.9 \pm 1.2) \cdot 10^{-8}$ \\
\hline$\chi_{c 2}(1 P)$ & $3556.18 \pm 0.13$ & $(6.8 \pm 0.7) \cdot 10^{-5}$ & - & - \\
\hline$\psi(2 S)$ & $3685.96 \pm 0.09$ & $(2.07 \pm 0.31) \cdot 10^{-4}$ & $(6.8 \pm 0.4) \cdot 10^{-4}$ & $(1.41 \pm 0.23) \cdot 10^{-7}$ \\
\hline \hline
\end{tabular}

\subsection{Event Selection}

The event selection is optimized [144] to maximize the statistical sensitivity of the $B^{+} \rightarrow p \bar{p} K^{+}$signal, defined as $S / \sqrt{S+B}$, with $S$ and $B$ being estimated numbers of $B^{+} \rightarrow p \bar{p} K^{+}$signal and background yields in the Monte Carlo simulation, respectively, and assuming the $B^{+} \rightarrow p \bar{p} K^{+}$signal branching fraction of $5.7 \times 10^{-6}$ [131]. The Fisher Discriminant cut $(\mathcal{F}>0.6)$ shown in Figure $3.4(\mathrm{~b})$ retains $66 \%$ of $B^{+} \rightarrow p \bar{p} K^{+}$signal events while removing $93 \%$ of continuum background. The resulting distribution of events in the $m_{E S}-\Delta E$ plane is shown in Figure 3.5. The signal and sideband regions are defined to be "wide" $\left(5.27<m_{E S}<5.29 \mathrm{GeV} / c^{2}\right.$ and $5.20<m_{E S}<5.26 \mathrm{GeV} / c^{2},|\Delta E|<50 \mathrm{MeV}$ ) for the charmonium background studies and "narrow" $\left(5.276<m_{E S}<5.286 \mathrm{GeV} / c^{2}\right.$ and $\left.5.20<m_{E S}<5.26 \mathrm{GeV} / c^{2},|\Delta E|<29 \mathrm{MeV}\right)$ for the Dalitz plot study.

\subsection{Branching fraction measurement}

A fit to the $\Delta E$ projection $\left(5.27<m_{E S}<5.29 \mathrm{GeV} / c^{2}\right)$ of all data (see Figure 3.6(a)) with a first order polynomial and a single Gaussian yields $643 \pm 36$ signal events. Fitting the $m_{E S}$ distribution (for $|\Delta E|<50 \mathrm{MeV}$ ) with a single Gaussian for the signal and an ARGUS function for the background shape (see Figure 3.6(b)), we obtain $663 \pm 39$ events. The number of background events in the wide sideband region $\left(5.20<m_{E S}<5.26 \mathrm{GeV} / \mathrm{c}^{2}\right.$ and $\left.|\Delta E|<50 \mathrm{MeV}\right)$ is 2870 events, corresponding to 569 combinatoric background events in the wide signal region $\left(5.27<m_{E S}<\right.$ 


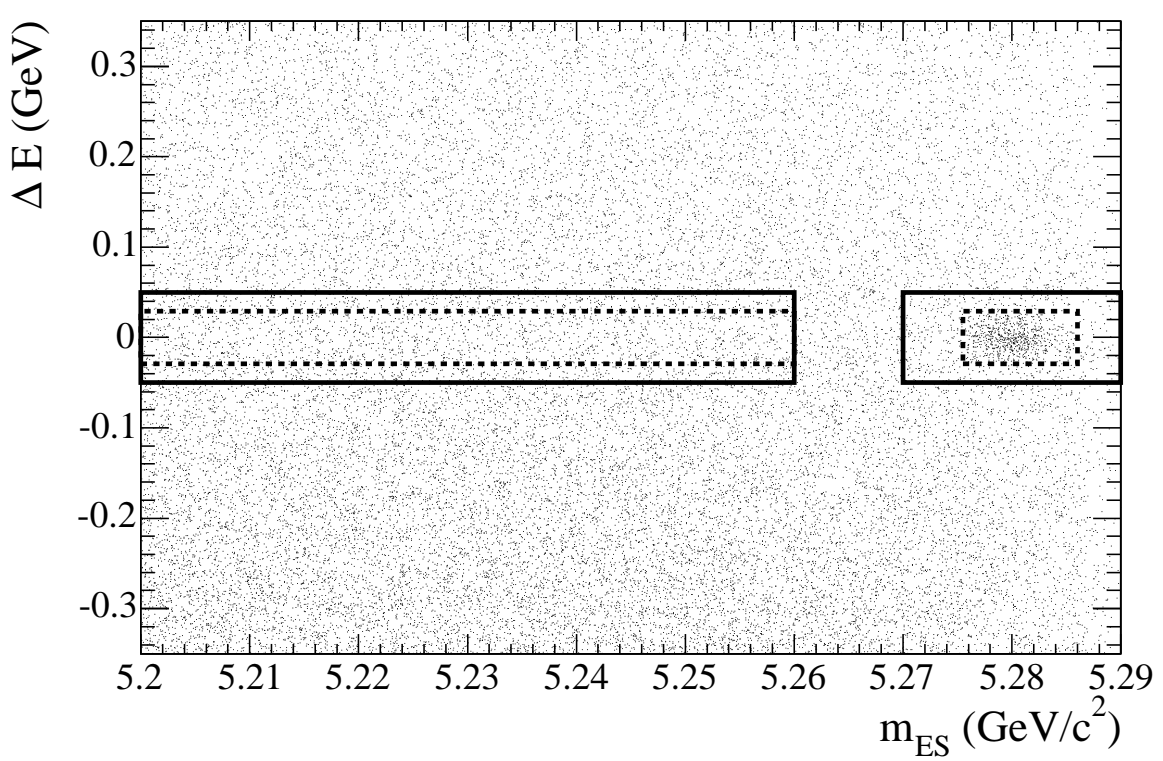

Figure 3.5: Distribution of $\Delta E$ versus $m_{E S}$ for the $p \bar{p} K^{+}$candidates in on-resonance data. The solid (dashed) lines define the wide (narrow) signal and sideband regions.
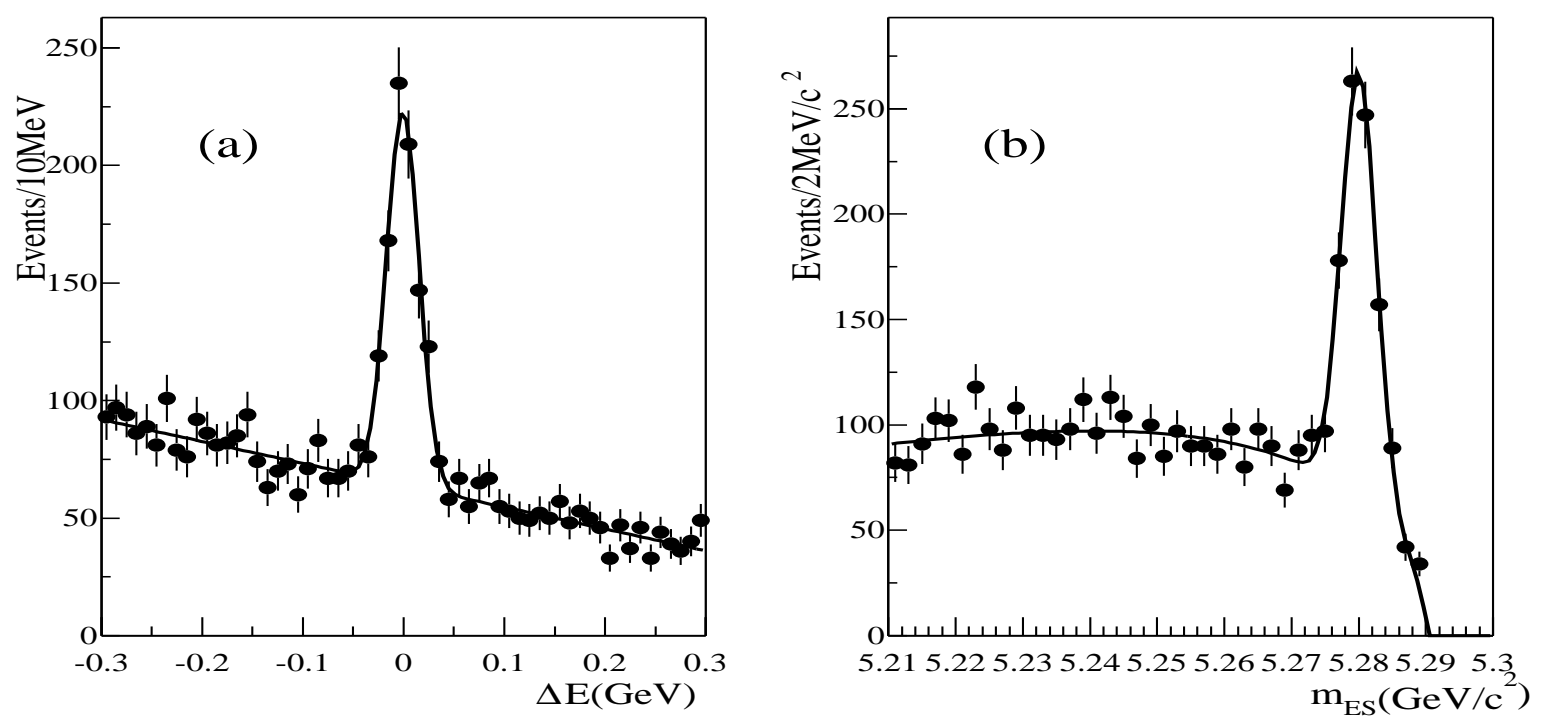

Figure 3.6: (a) $\Delta E$ distribution of on-peak data $\left(5.27<m_{E S}<5.29 \mathrm{GeV} / c^{2}\right)$; (b) $m_{E S}$ distribution of on-peak data $(|\Delta E|<0.05 \mathrm{GeV})$. 

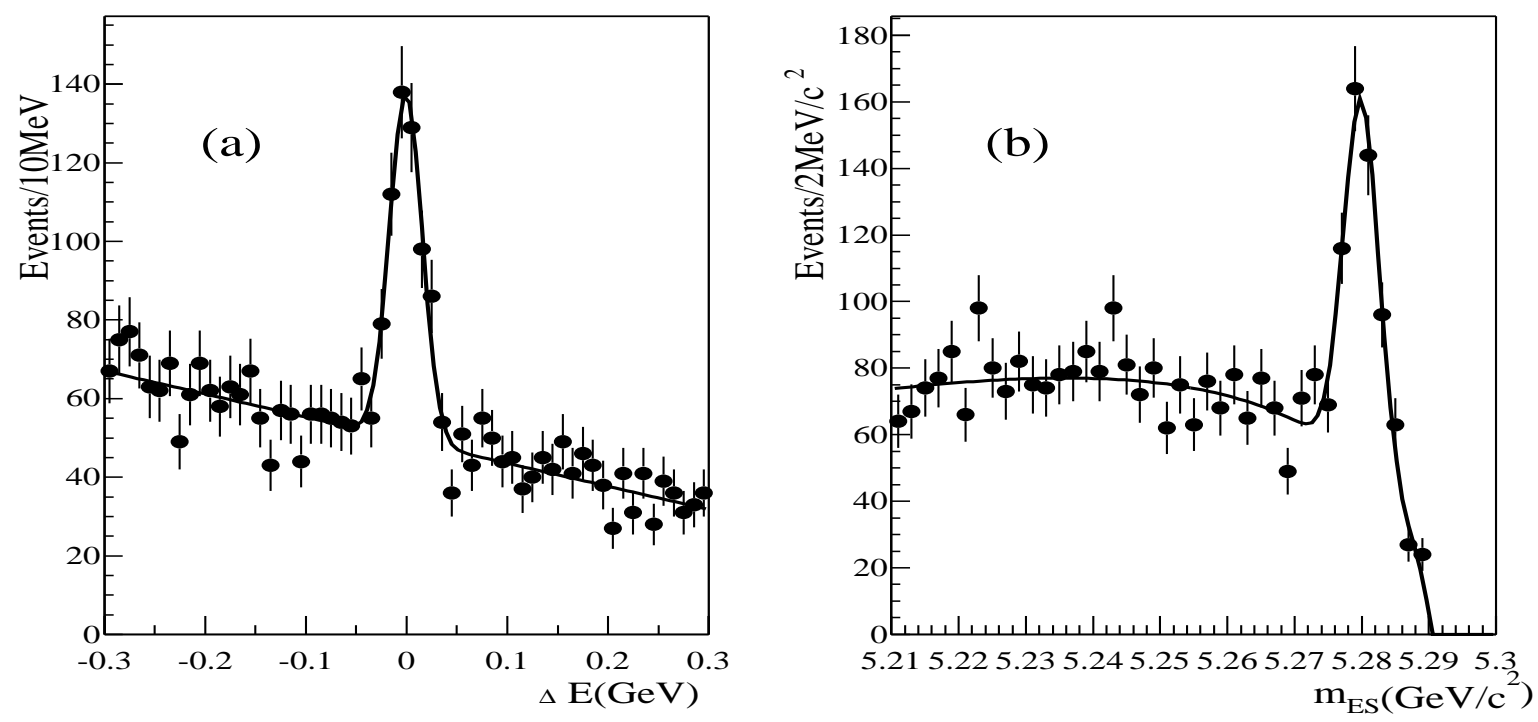

Figure 3.7: (a) $\Delta E$ distribution of on-peak data $\left(5.27<m_{E S}<5.29 \mathrm{GeV} / \mathrm{c}^{2}\right)$; (b) $m_{E S}$ distribution of on-peak data $(|\Delta E|<0.05 \mathrm{GeV})$. Note that we veto charmonium contribution: by requiring $m_{p \bar{p}}<2.85 \mathrm{GeV} / \mathrm{c}^{2}, 3.128<m_{p \bar{p}}<3.315 \mathrm{GeV} / \mathrm{c}^{2}$ and $\left.m_{p \bar{p}}>3.735 \mathrm{GeV} / \mathrm{c}^{2}\right)$.

$5.29 \mathrm{GeV} / \mathrm{c}^{2}$ and $\left.|\Delta E|<50 \mathrm{MeV}\right)$; thus the relative phase-space, or the fraction of the combinatoric background in the wide signal box to the number of events in the wide sideband, is 0.198. If we remove the charmonium region $\left(2.85<m_{p \bar{p}}<3.128 \mathrm{GeV} / \mathrm{c}^{2}\right.$, $\left.3.315<m_{p \bar{p}}<3.735 \mathrm{GeV} / \mathrm{c}^{2}\right)$ we obtain $358 \pm 24$ events from the $\Delta E$ fit and $365 \pm 32$ events from the $m_{E S}$ fit (see Figure 3.7).

As the signal shape is expected to be different from the shape predicted by the phase-space Monte-Carlo we cannot a priori estimate the total signal efficiency. Instead we calculate the efficiency in bins of $m_{p \bar{p}}$ and correct the observed events, bin by bin. The branching fraction can be calculated as follows:

$$
\mathcal{B}\left(B^{+} \rightarrow p \bar{p} K^{+}\right)=\left(\sum \frac{N_{m_{p \bar{p}}}}{\epsilon_{m_{p \bar{p}}}}\right) / N_{B}
$$

where $N_{m_{p \bar{p}}}$ is the number of events, $\epsilon_{m_{p \bar{p}}}$ is the efficiency in each of the $m_{p \bar{p}}$ bins and 


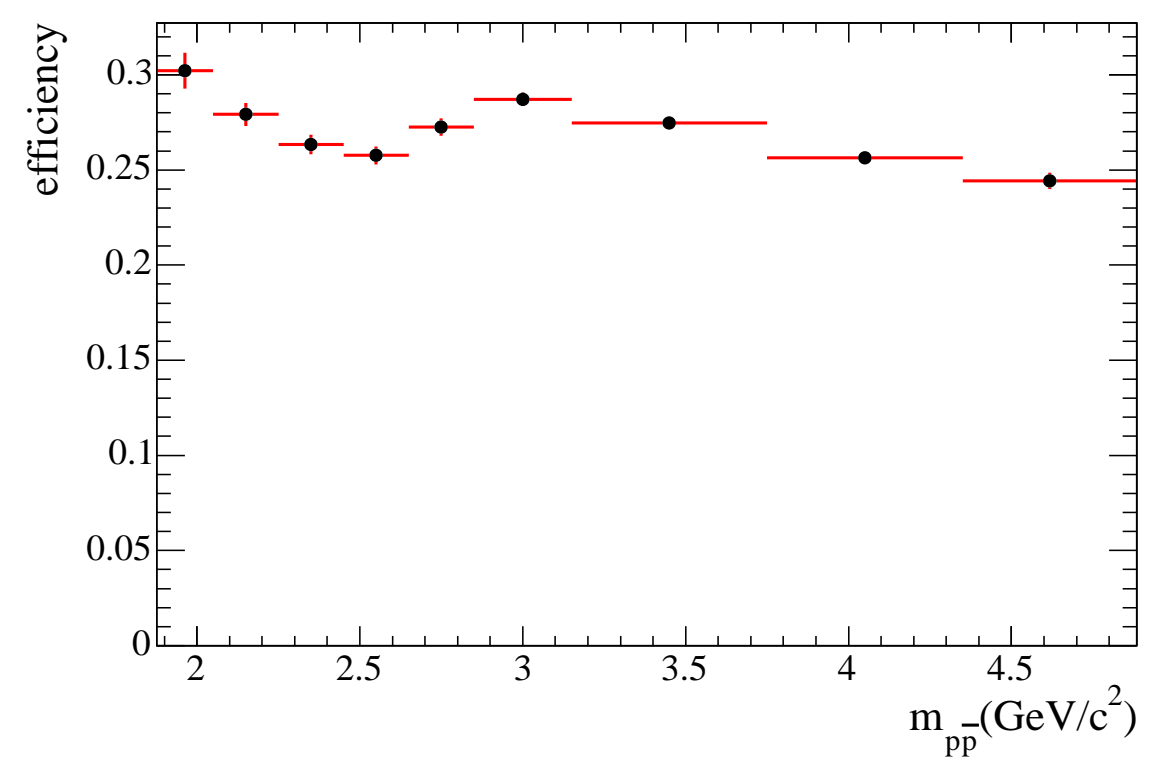

Figure 3.8: Reconstructed efficiency of $B^{+} \rightarrow p \bar{p} K^{+}$signal events as a function of $m_{p \bar{p}}$.

$N_{B}=232 \times 10^{6}$ is the number of charged $B$ mesons in the data sample.

To extract the $p \bar{p} K^{+}$signal yield, we fit the $\Delta E$ distributions of the candidates that lie in the $5.27<m_{E S}<5.29 \mathrm{GeV} / c^{2}$ region, separately in nine bins of $m_{p \bar{p}}$ (see Figure 3.9). The width and location of each bin are shown in Figure 3.10. We use a linear function for the background and a double Gaussian distribution for the signal. The widths and means of the Gaussian distributions and their relative areas are fixed to values obtained from $\mathrm{MC}$ simulation, which is also used to calculate the detection efficiency $\left(\varepsilon_{m_{p \bar{p}}}\right)$ in each $m_{p \bar{p}}$ bin. As shown in Figure 3.8, $\varepsilon_{m_{p \bar{p}}}$ declines smoothly from $30 \%$ at threshold to $24 \%$ at the highest kinematically allowed mass. Fit results for the $\Delta E$ distribution are given in Table 3.2 for on-peak data.

\subsection{1 $\mathcal{B}\left(B^{+} \rightarrow p \bar{p} K^{+}, m_{p \bar{p}}<2.85 \mathrm{GeV} / c^{2}\right)$ measurement}

Summing the $\Delta E$ fits for $m_{p \bar{p}}$ below $2.85 \mathrm{GeV} / c^{2}$ yields $343_{-26}^{+27}$ signal events (see Table 3.2). From the known number of charged $B$ mesons in the sample, the branching 

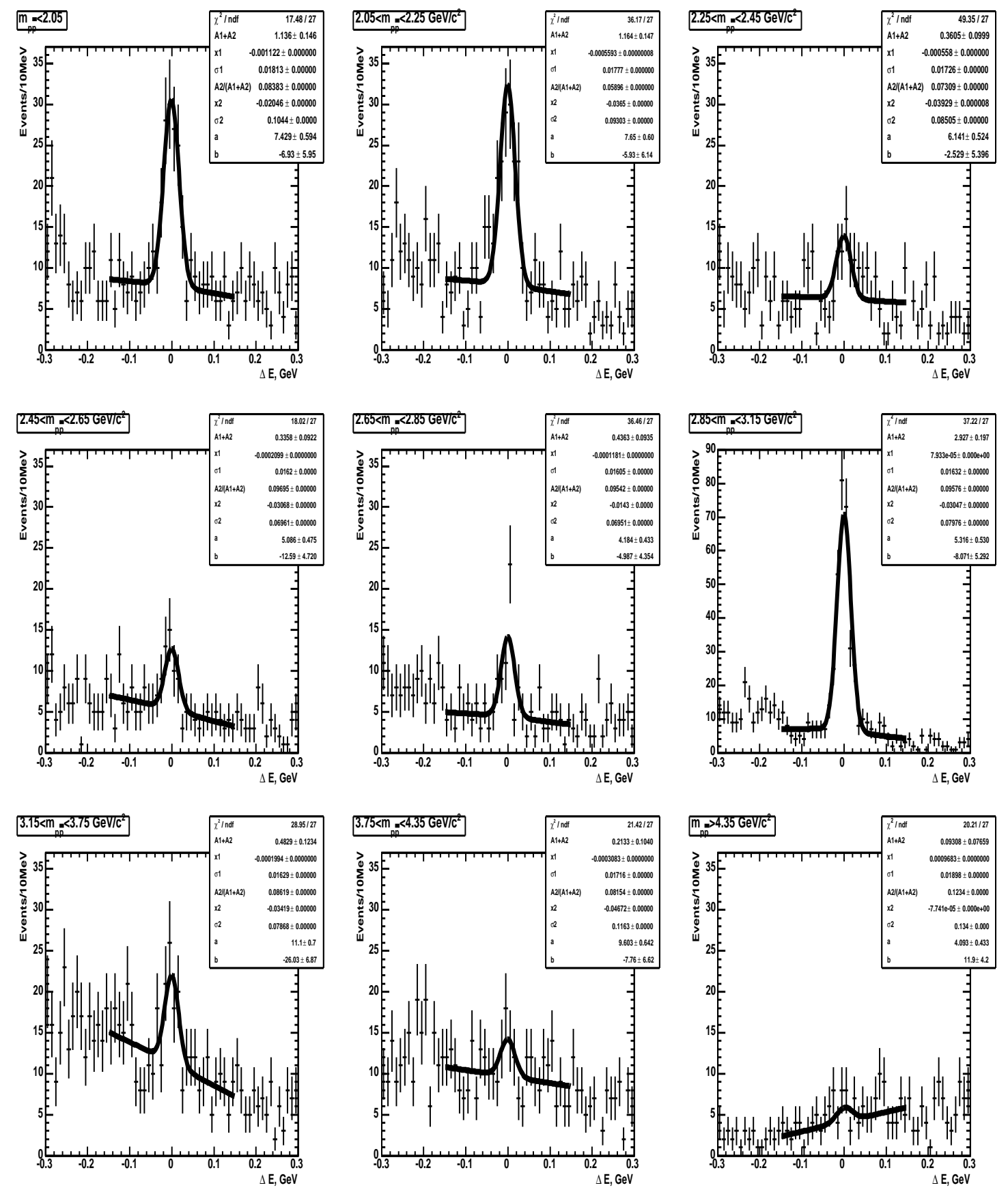

Figure 3.9: $\Delta E$ distribution for on-peak data in different $m_{p \bar{p}}$ regions for $B^{+} \rightarrow p \bar{p} K^{+}$. 
Table 3.2: $\Delta E$ fit results used for the $B^{+} \rightarrow p \bar{p} K^{+}$branching fraction calculation.

\begin{tabular}{||c|c|c|c|c||}
\hline \hline Mass Regions, GeV/c $c^{2}$ & $N_{m_{p \bar{p}}}$ & $\epsilon_{m_{p \bar{p}}}, \%$ & Eff.Cor.Events & $\mathcal{B}, 10^{-6}$ \\
\hline \hline $1.85<m_{p p}<2.05$ & $113.6_{-14.2}^{+14.9}$ & $30.2 \pm 0.9$ & $376.2_{-47.0}^{+49.3}$ & $1.62_{-0.20}^{+0.21}$ \\
$2.05<m_{p p}<2.25$ & $116.4_{-14.4}^{+15.0}$ & $27.9 \pm 0.6$ & $417.2_{-51.6}^{+53.8}$ & $1.80_{-0.22}^{+0.23}$ \\
$2.25<m_{p p}<2.45$ & $36.1_{-9.6}^{+10.3}$ & $26.3 \pm 0.5$ & $137.3_{-36.5}^{+39.2}$ & $0.59_{-0.16}^{+0.17}$ \\
$2.45<m_{p p}<2.65$ & $33.6_{-8.9}^{+9.6}$ & $25.8 \pm 0.5$ & $130.2_{-34.5}^{+37.2}$ & $0.56_{-0.15}^{+0.16}$ \\
$2.65<m_{p p}<2.85$ & $43.6_{-9.0}^{+9.7}$ & $27.3 \pm 0.5$ & $159.7_{-33.0}^{+35.5}$ & $0.69_{-0.14}^{+0.15}$ \\
\hline $3.15<m_{p p}<3.75$ & $48.3_{-12.0}^{+12.7}$ & $27.5 \pm 0.3$ & $175.6_{-43.6}^{+46.2}$ & $0.76_{-0.19}^{+0.20}$ \\
$3.75<m_{p p}<4.35$ & $21.3_{-10.0}^{+10.8}$ & $25.6 \pm 0.3$ & $83.2_{-39.1}^{+42.2}$ & $0.36_{-0.17}^{+0.18}$ \\
$4.35<m_{p p}$ & $9.3 \pm 7.7$ & $24.4 \pm 0.4$ & $38.1 \pm 31.6$ & $0.16 \pm 0.14$ \\
\hline Sum of fits $m_{p \bar{p}}<2.85 \mathrm{GeV}$ & $343.3_{-25.7}^{+27.1}$ & - & $1220.6_{-92.1}^{+99.5}$ & $5.26_{-0.40}^{+0.42}$ \\
\hline Sum of all fits & $422.2_{-31.1}^{+32.8}$ & - & $1517.5_{-113.6}^{+120.1}$ & $6.54_{-0.49}^{+0.52}$ \\
\hline \hline
\end{tabular}

fraction for $m_{p \bar{p}}$ below the $\eta_{c}$ mass is measured to be $\mathcal{B}\left(B^{+} \rightarrow p \bar{p} K^{+} ; m_{p \bar{p}}<2.85 \mathrm{GeV} / c^{2}\right)=$ $(5.3 \pm 0.4 \pm 0.3) \times 10^{-6}$.

\subsection{2 $\mathcal{B}\left(B^{+} \rightarrow \eta_{c} K^{+}\right) \times \mathcal{B}\left(\eta_{c} \rightarrow p \bar{p}\right)$ and $\Gamma\left(\eta_{c}\right)$ measurements}

Measurement of the charmonium contribution in the $m_{p \bar{p}}>2.85 \mathrm{GeV} / c^{2}$ region is required to determine the total non-charmonium $B^{+} \rightarrow p \bar{p} K^{+}$branching fraction. To minimize the systematic error on that quantity, we fit the $m_{p \bar{p}}$ spectrum for the number of the non-charmonium events in the primary charmonium region $(2.85<$ $\left.m_{p \bar{p}}<3.15 \mathrm{GeV} / c^{2}\right)$. To improve the $p \bar{p}$ mass resolution in the $m_{p \bar{p}}$ fit, we perform a kinematic fit fixing the mass and energy of each $B$ candidate in the wide signal and sideband regions to their known values. The $m_{p \bar{p}}$ distribution is shown in Figure 3.10, where prominent signals for the $\eta_{c}$ and $J / \psi$ decaying into $p \bar{p}$ are visible. The region used in the $m_{p \bar{p}}$ fit, $2.4<m_{p \bar{p}}<3.4 \mathrm{GeV} / c^{2}$, is chosen wider than the primary charmonium region, shown in Figure 3.10(inset), to improve the statistical uncertainties on the $p \bar{p} K^{+}$signal and combinatorial background yield. The $\eta_{c}$ peak is described by a convolution of a Breit-Wigner line-shape and a Gaussian distribution, and the $J / \psi$ peak by a sum of two Gaussian distributions with a common 


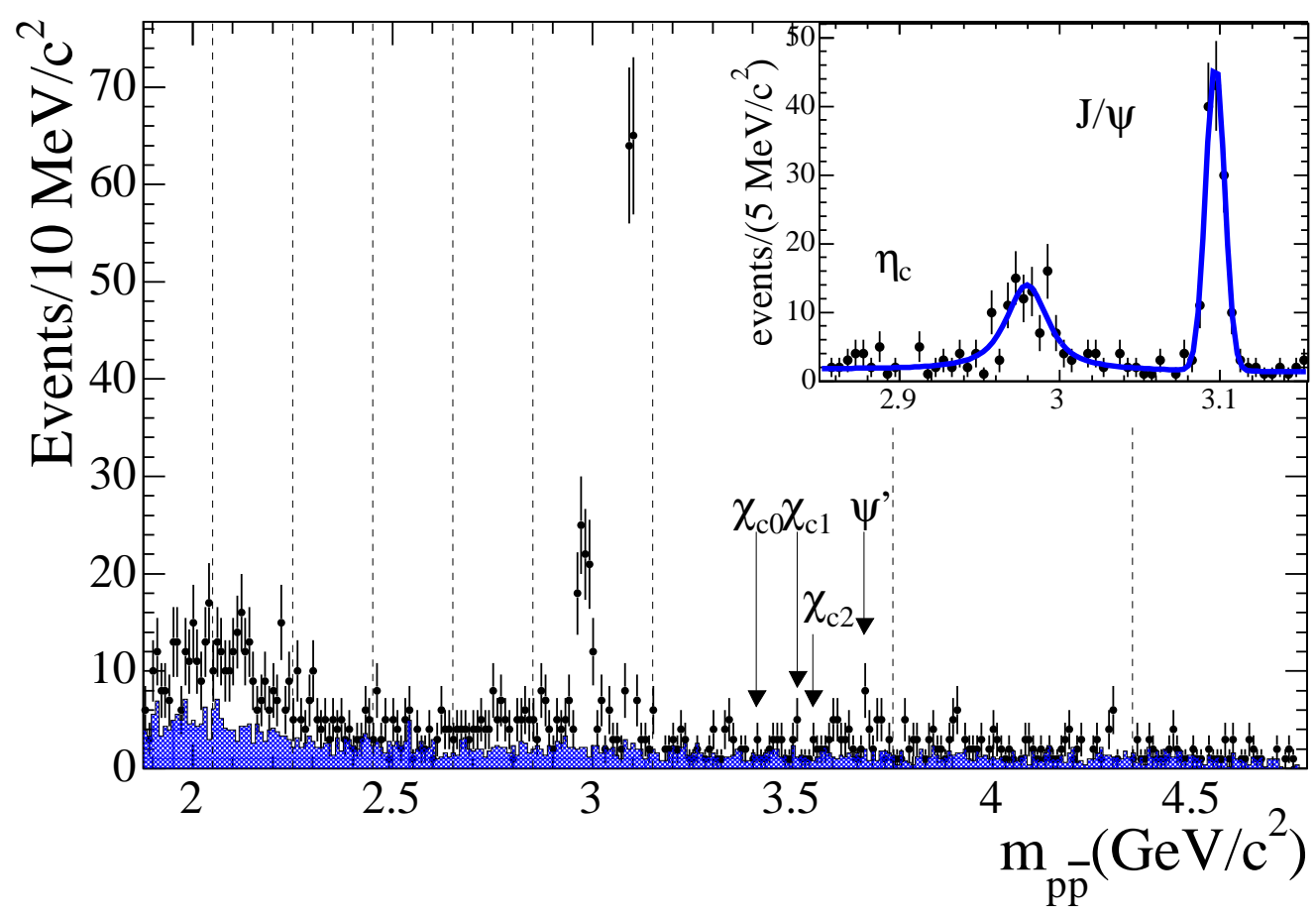

Figure 3.10: The $m_{p \bar{p}}$ distribution for data in the wide signal (points) and sideband (shaded) regions. The sideband histogram is scaled to the expected number of the combinatorial background events in the signal region. 
mean. The shapes are obtained from MC simulation. The width of the broader $J / \psi$ Gaussian distribution and ratio of areas of the two $J / \psi$ Gaussian distributions are constrained in the fit to their MC values. A common width is used for the narrow Gaussian distributions for $J / \psi$ and $\eta_{c}$ and is a free parameter in the fit. The $p \bar{p} K^{+}$signal and combinatorial background distributions are modeled by a linear function of $m_{p \bar{p}}$. The inset of Figure 3.10 shows the result of the fit, which yields $114_{-14}^{+15} \eta_{c}$ events and $137_{-12}^{+13} \mathrm{~J} / \psi$ events. Correcting for the detection efficiency of $(26.9 \pm 0.2) \%$, we find $\mathcal{B}\left(B^{+} \rightarrow \eta_{c} K^{+}\right) \times \mathcal{B}\left(\eta_{c} \rightarrow p \bar{p}\right)=\left(1.8_{-0.2}^{+0.3} \pm 0.2\right) \times 10^{-6}$ and $\mathcal{B}\left(B^{+} \rightarrow J / \psi K^{+}\right) \times \mathcal{B}(J / \psi \rightarrow p \bar{p})=(2.2 \pm 0.2 \pm 0.1) \times 10^{-6}$ in agreement with the PDG values [20]. The fit yields a total $\eta_{c}$ width of $\Gamma\left(\eta_{c}\right)=25_{-5}^{+6} \pm 3 \mathrm{MeV} / c^{2}$, consistent with the current values [20], and a mass resolution of $5.7 \pm 0.4 \mathrm{MeV} / c^{2}$, in agreement with MC expectations.

\subsubsection{Total $\mathcal{B}\left(B^{+} \rightarrow p \bar{p} K^{+}\right)$measurement}

The linear component of the $m_{p \bar{p}}$ fit yields $88 \pm 6 p \bar{p} K^{+}$signal and combinatorial background events in the primary charmonium region. In this region, the background contribution is estimated from the $\Delta E$ fit to be $53 \pm 5$ events, resulting in a non-charmonium $p \bar{p} K^{+}$signal of $35 \pm 8$ events. The $\Delta E$ fits for $m_{p \bar{p}}>3.15 \mathrm{GeV} / c^{2}$ yields $79 \pm 18$ signal events including the contribution from higher-mass charmonium modes. We estimate the latter to be $24 \pm 5$ events, using their measured [20] branching fractions. Adding the $p \bar{p} K^{+}$signal yield obtained from the $\Delta E$ fits outside the primary charmonium region ( $422 \pm 32$ events) with non-charmonium $p \bar{p} K^{+}$signal inside the primary charmonium region ( $35 \pm 8$ events), and subtracting the contribution of the higher mass charmonium modes ( $24 \pm 5$ events) results in a total non-charmonium signal yield of $433 \pm 33$ events. Correcting the signal yield for efficiency in each of the $m_{p \bar{p}}$ bins and normalizing to the number of $B^{+}$mesons in the data sample $\left(232 \times 10^{6}\right)$ results in a total branching fraction of $\mathcal{B}\left(B^{+} \rightarrow p \bar{p} K^{+}\right)=(6.7 \pm 0.5 \pm 0.4) \times 10^{-6}$ where charmonium decays to $p \bar{p}$ are excluded.

The observed $p \bar{p}$ mass spectrum (Figure 3.11) differs from a phase-space distribution; it peaks dramatically at low $p \bar{p}$ mass. To remove the kinematic threshold 


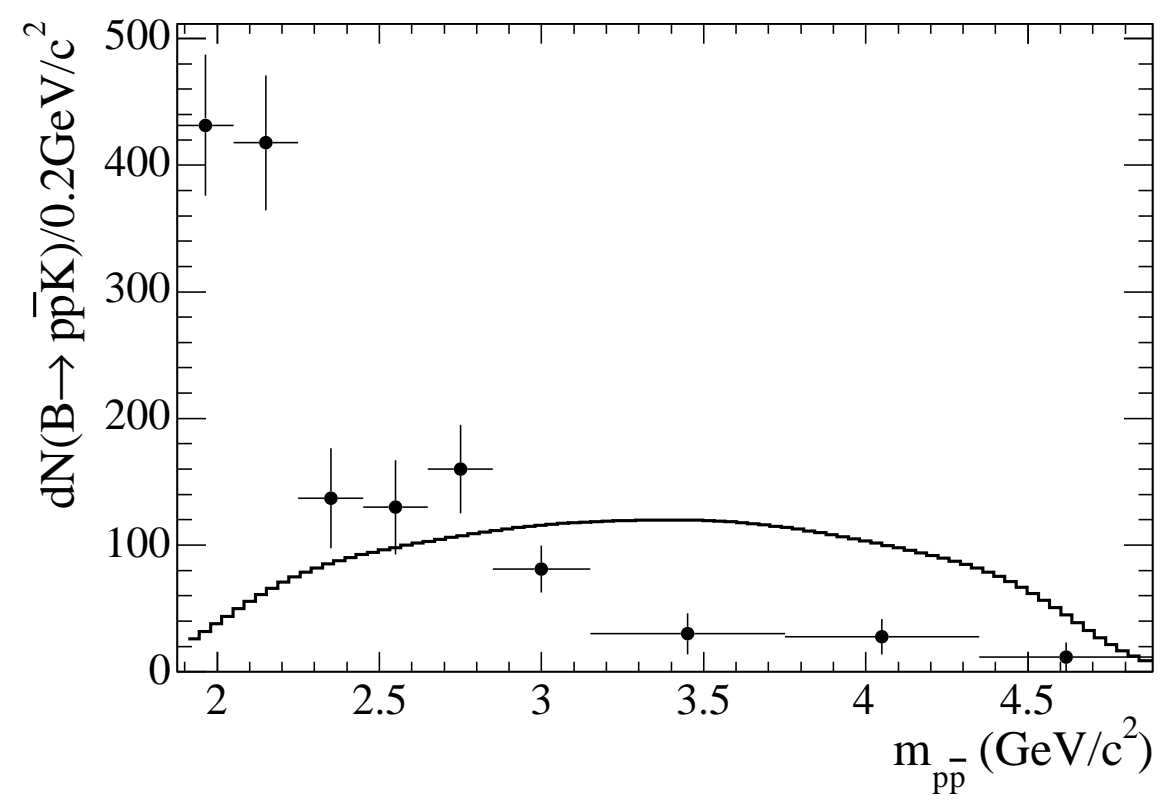

Figure 3.11: Efficiency-corrected yield of $B^{+} \rightarrow p \bar{p} K^{+}$events as a function of $m_{p \bar{p}}$ in data (points) and in three-body phase-space signal MC (histogram). Errors shown are statistical only. 

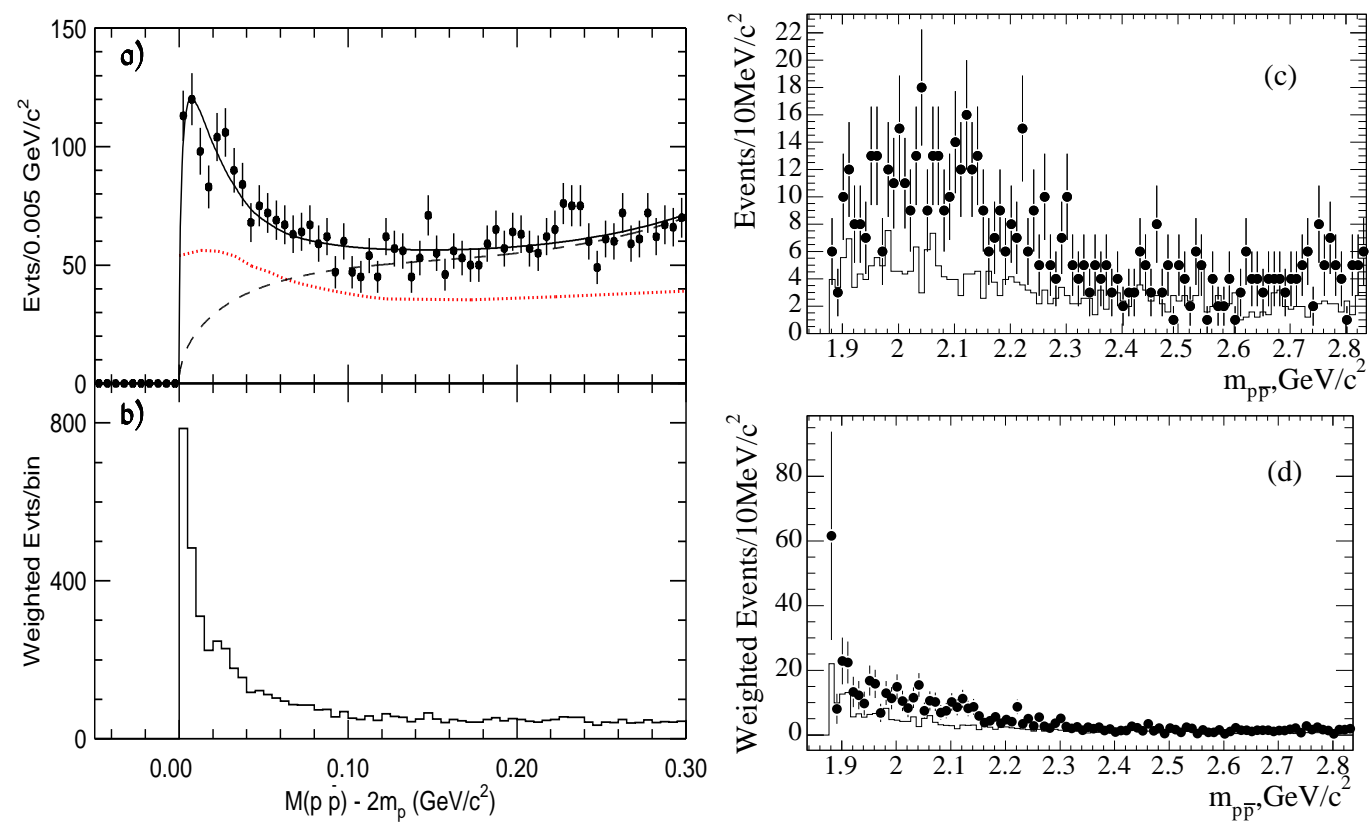

Figure 3.12: (a) The near threshold enhancement observed in $M_{p \bar{p}}-2 m_{p}$ distribution for the $J / \psi \rightarrow \gamma p \bar{p}$ event sample [34]. The dashed curve is the background function described in the text. The dotted curve indicates how the acceptance varies with $p \bar{p}$ invariant mass; the dashed curve shows the fitted background function. (b) The same $M_{p \bar{p}}-2 m_{p}$ distribution with events weighted by $q_{0} / q$. (c) Near threshold distribution of $m_{p \bar{p}}$ for the $p \bar{p} K^{+}$event sample (in the wide signal box region). The histogram is the background estimation from the wide sideband. (d) The same $m_{p \bar{p}}$ distribution in the $B^{+} \rightarrow p \bar{p} K^{+}$sample with events weighted by $q_{0} / q$. Note that distributions (c) and $(\mathrm{d})$ are not efficiency corrected, but the efficiency is expected to be approximately flat. 


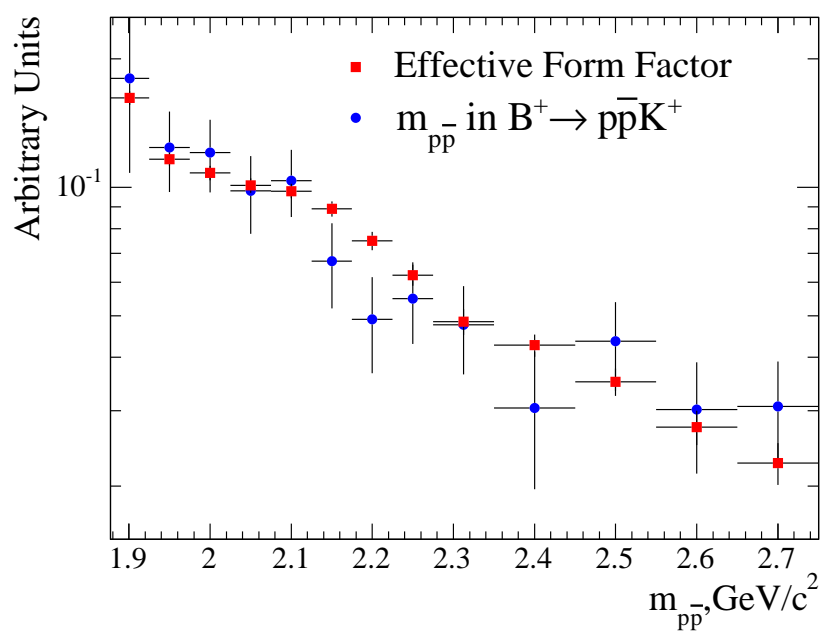

Figure 3.13: Phase-space-corrected $m_{p \bar{p}}$ distribution of $B^{+} \rightarrow p \bar{p} K^{+}$signal events (blue dots) compared to the effective form factor from $e^{+} e^{-} \gamma \rightarrow \gamma p \bar{p}$ [145] (red squares). Note that $B^{+} \rightarrow p \bar{p} K^{+}$distribution is not efficiency corrected, but the efficiency is expected to be approximately flat.

behavior each event is weighted by $q_{0} / q$, where $q$ is the proton momentum in the $p \bar{p}$ rest-frame and $q_{0}$ is the value at fixed $m_{p \bar{p}}\left(2 \mathrm{GeV} / \mathrm{c}^{2}\right.$ here). The weighted and unweighted events as well as a background estimated from sideband data are shown in Figure 3.12(c). There is a sharp and monotonic increase at threshold observed in the weighted histogram which is consistent with the one seen by BES in $J / \psi \rightarrow p \bar{p} \gamma[34]$ in Figure 3.12(d). Threshold behavior of the phase-space corrected ${ }^{3} m_{p \bar{p}}$ distribution is also compared to the effective form factor as measured in $e^{+} e^{-} \gamma \rightarrow \gamma p \bar{p}[145]$ (Figure 3.13). It is striking that those two different kinematic and dynamic processes have similar distributions in the $p \bar{p}$ invariant mass.

\subsubsection{Measurement of the charge asymmetry in $B^{+} \rightarrow p \bar{p} K^{+}$}

Removing extra quark lines from Figures $1.4(\mathrm{a}, \mathrm{b}, \mathrm{e})$ one can relate $B^{+} \rightarrow p \bar{p} K^{+}$to corresponding penguin and tree diagrams of $B^{+} \rightarrow K^{+} \pi^{0}$. After observation of the

\footnotetext{
${ }^{3}$ Phase-space correction means that the distribution is weighted by $q_{0} \cdot p_{0} /(q \cdot p)$, where $q$ is the proton momentum in the $p \bar{p}$ frame, $q_{0}$ its value at $m_{p \bar{p}}=2 \mathrm{GeV} / c^{2}$ and $p$ is the kaon momentum in $B$ rest frame, $p_{0}$ its value at $m_{p \bar{p}}=2 \mathrm{GeV} / c^{2}$ (the choice of $m_{p \bar{p}}=2 \mathrm{GeV} / c^{2}$ is arbitrary).
} 
Table 3.3: Summary of the $B^{+} \rightarrow p \bar{p} K^{+}$asymmetry study.

\begin{tabular}{||l|r|r|r||}
\hline \hline Region & $N_{B^{-}}$ & $N_{B^{+}}$ & $A_{c h}$ \\
\hline$m_{p \bar{p}}<2.85 \mathrm{GeV} / c^{2}$ & $143_{-17}^{+19}$ & $199_{-19}^{+20}$ & $-0.16_{-0.08}^{+0.07}$ \\
all $m_{p \bar{p}}$ & $203_{-23}^{+22}$ & $261_{-23}^{+25}$ & $-0.13_{-0.07}^{+0.08}$ \\
\hline \hline
\end{tabular}

direct CP violation in $B^{0} \rightarrow K^{+} \pi^{-}$[134] and its non-observation in $B^{+} \rightarrow K^{+} \pi^{0}$ [135], it would also be interesting to examine the charge asymmetry in the $B^{+} \rightarrow p \bar{p} K^{+}$ system.

The charge asymmetry is defined as $A_{c h}=\left(N_{B^{-}}-N_{B^{+}}\right) /\left(N_{B^{-}}+N_{B^{+}}\right)$, where $N_{B^{ \pm}}$ is the number of $B^{ \pm} \rightarrow p \bar{p} K^{ \pm}$events (see Table 3.3). We use the same fitting procedure as for the branching fraction measurement, and find $A_{c h}=-0.16_{-0.08}^{+0.07} \pm 0.04$ for $m_{p \bar{p}}<2.85 \mathrm{GeV} / c^{2}$ and $-0.13_{-0.07}^{+0.08} \pm 0.04$ for all $m_{p \bar{p}}$ range (excluding $\eta_{c}$ and $J / \psi$ contributions).

\subsubsection{Search for $B^{+} \rightarrow p \bar{\Lambda}(1520)$}

If the branching fraction of $B^{+} \rightarrow p \bar{\Lambda}(1520)$ is sufficiently large, we expect to see a concentration of $B^{+} \rightarrow p \bar{p} K^{+}$signal events at $m_{\bar{p} K^{+}}=1.52 \mathrm{GeV} / \mathrm{c}^{2}$. With $\mathcal{B}(\Lambda(1520) \rightarrow$ $N \bar{K})=45 \pm 1 \%$, and assuming isospin symmetry, $50 \%$ of those events would decay into $p K^{+}$. The signal efficiency after tracking and particle ID corrections is $20.8 \pm 0.2 \%$. The "combinatoric" background level to a $\Lambda(1520)$ signal can be seen in see Figure 3.14.

The $m_{\bar{p} K^{+}}$spectrum, shown in Figure 3.15(a), is fit with an ARGUS function [141] for the background and a Breit-Wigner convolved with a double Gaussian (with a common mean) for the $\Lambda(1520)$ signal. The mass resolutions and the ratio of areas of the Gaussians are fixed to the values obtained from MC simulation ${ }^{4}$, and the mean and the natural width to established values [20]; the endpoint of the ARGUS function is fixed to the sum of the proton and kaon masses. An unbinned maximum likelihood

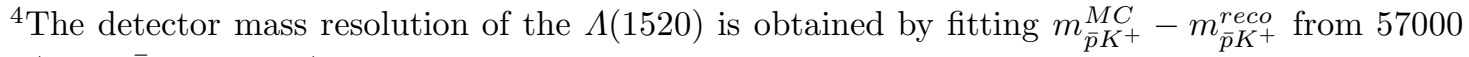
of $B^{+} \rightarrow p \bar{\Lambda}(1520)\left[\bar{p} K^{+}\right]$Monte Carlo events to a sum of two Gaussian distributions (with common mean). The parameters of the Gaussian distributions are widths $\sigma_{1}=0.78 \pm 0.03 \mathrm{MeV}$ and $\sigma_{2}=$ $1.61 \pm 0.08 \mathrm{MeV}$ and the relative area of the two Gaussians of $0.436 \pm 0.098$.
} 


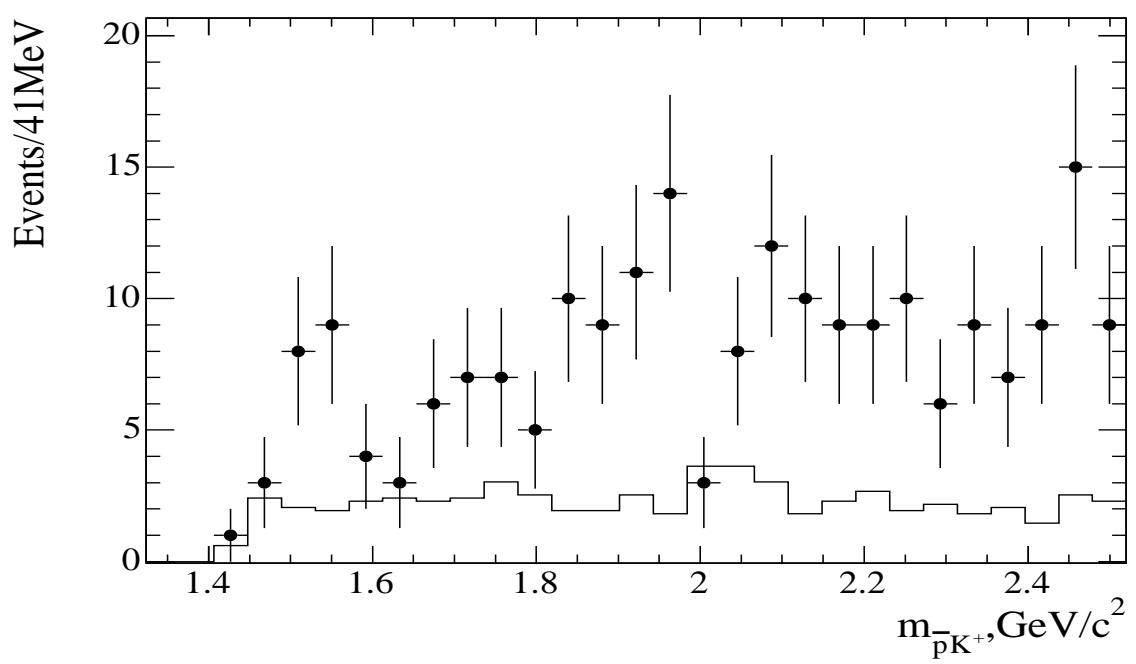

Figure 3.14: The $m_{\bar{p} K^{+}}$distribution for data events (points) in $B^{+} \rightarrow p \bar{p} K^{+}$signal box and $1(1520)$ mass region, not efficiency corrected. The histogram is rescaled $m_{\bar{p} K^{+}}$distribution from $m_{E S}$ sideband region.

fit and the likelihood function for it are shown in Figure 3.15(a,b). The resulting branching fraction is $\left(0.61_{-0.54}^{+0.63}(\right.$ stat $) \pm 0.10$ (syst $\left.)\right) \times 10^{-6}$ which leads to an upper limit (U.L.) on $\mathcal{B}\left(B^{+} \rightarrow p \bar{\Lambda}(1520)\right)$ of $1.5 \times 10^{-6}$ at $90 \%$ C.L. (including a systematic error of $16 \%$ ).

\subsection{6 $\quad \Theta^{*++}$ pentaquark search}

As we are interested only in the low $m_{p K^{+}}$region, the following figures will be limited to $m_{p K^{+}}$up to $2 \mathrm{GeV} / \mathrm{c}^{2}$. There are the total of 25 events in the signal box in this $m_{p K^{+}}$ region. It is convenient to represent data in two distinct ways: assuming that the width of $\Theta^{*++}$ is negligible and assuming that $\Theta^{*++}$ has a width of $\sigma_{\Theta^{*++}}=10 \mathrm{MeV}$. The binning of the plots corresponds to $4 \cdot \sigma_{p K^{+}}$or $4 \cdot \sqrt{\sigma_{p K^{+}}^{2}+\sigma_{\Theta^{*++}}^{2}}$, where $\sigma_{p K^{+}}$is the detector resolution shown in Figure 3.16(a) and $\sigma_{\Theta^{*++}}^{2}=10 \mathrm{MeV}$.

We use two different MC samples to calculate the signal efficiency: 173k events of the standard $B^{+} \rightarrow p \bar{p} K^{+}$phase-space $\mathrm{MC}\left(\mathrm{MC}_{p s}\right)$ and 5 samples of $57 \mathrm{k}$ each, 

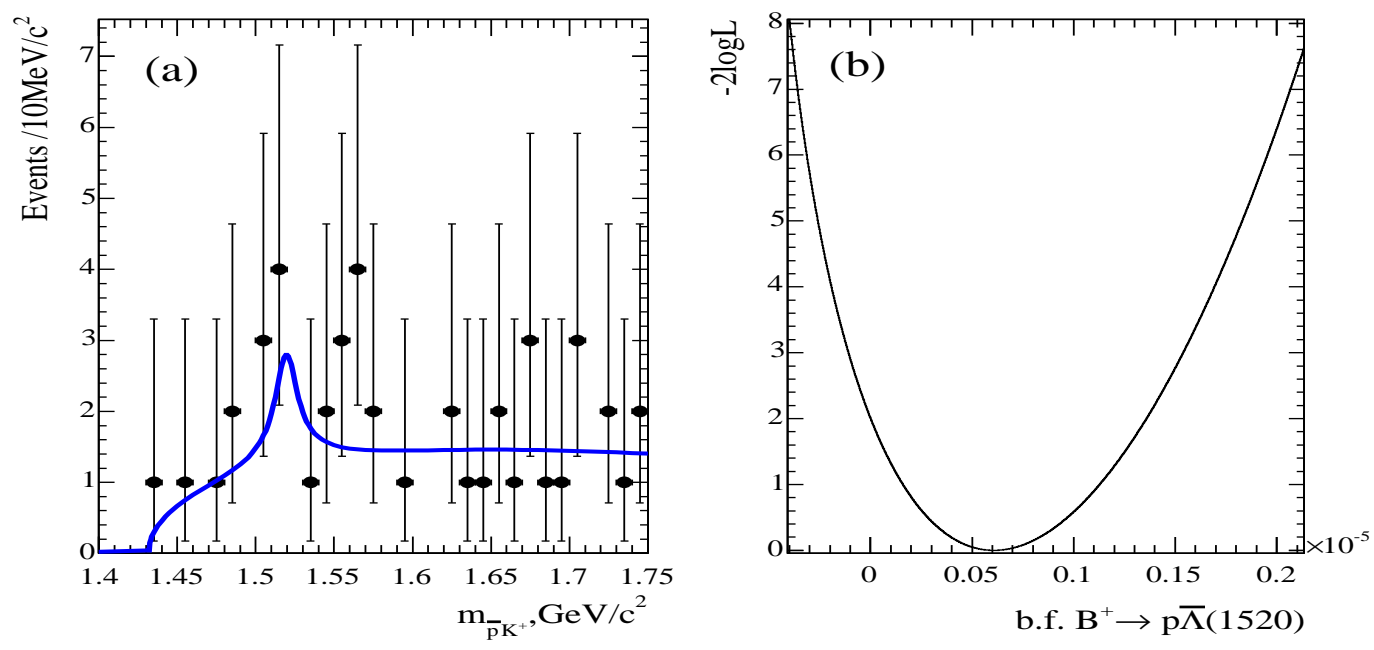

Figure 3.15: (a) The $m_{\bar{p} K^{+}}$distribution for data events in $B^{+} \rightarrow p \bar{p} K^{+}$signal box and $1(1520)$ mass region. Results of the maximum likelihood fit are overlaid. (b) Likelihood distribution as a function of the branching fraction of $B^{+} \rightarrow p \bar{\Lambda}(1520)$.
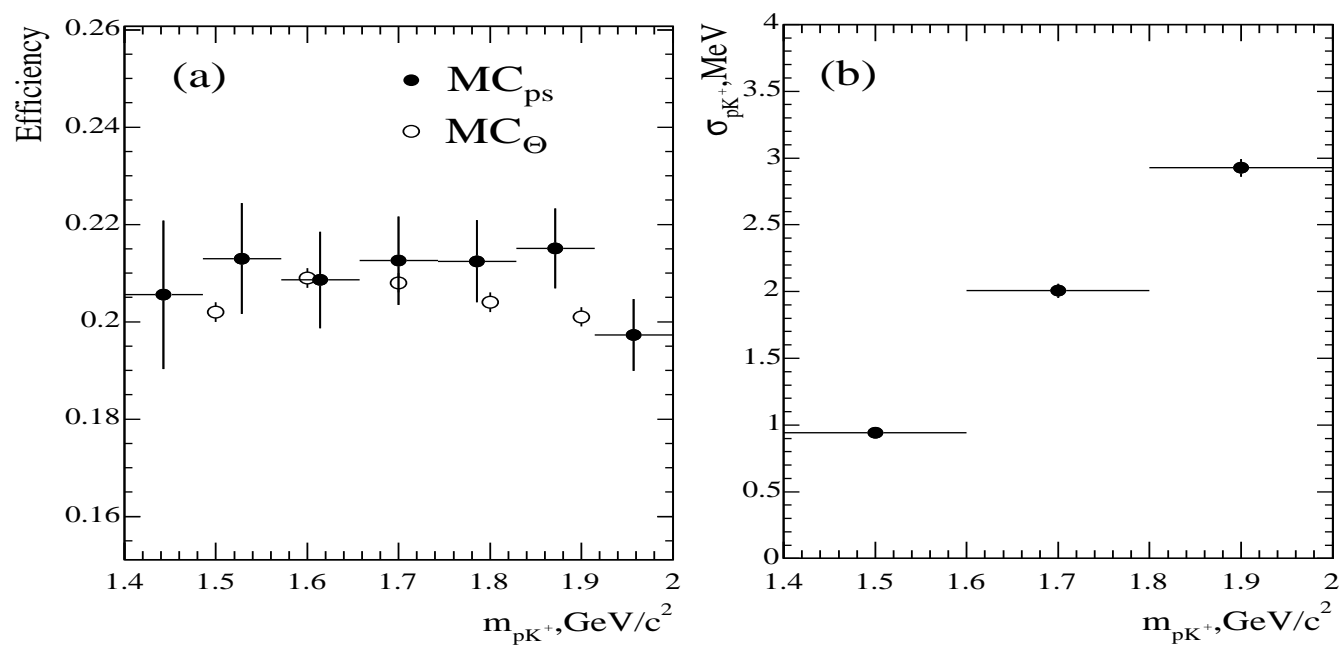

Figure 3.16: The $B^{+} \rightarrow p \bar{p} K^{+}$signal reconstruction efficiency (a) and the detector resolution (b) as functions of $m_{p K^{+}}$ 

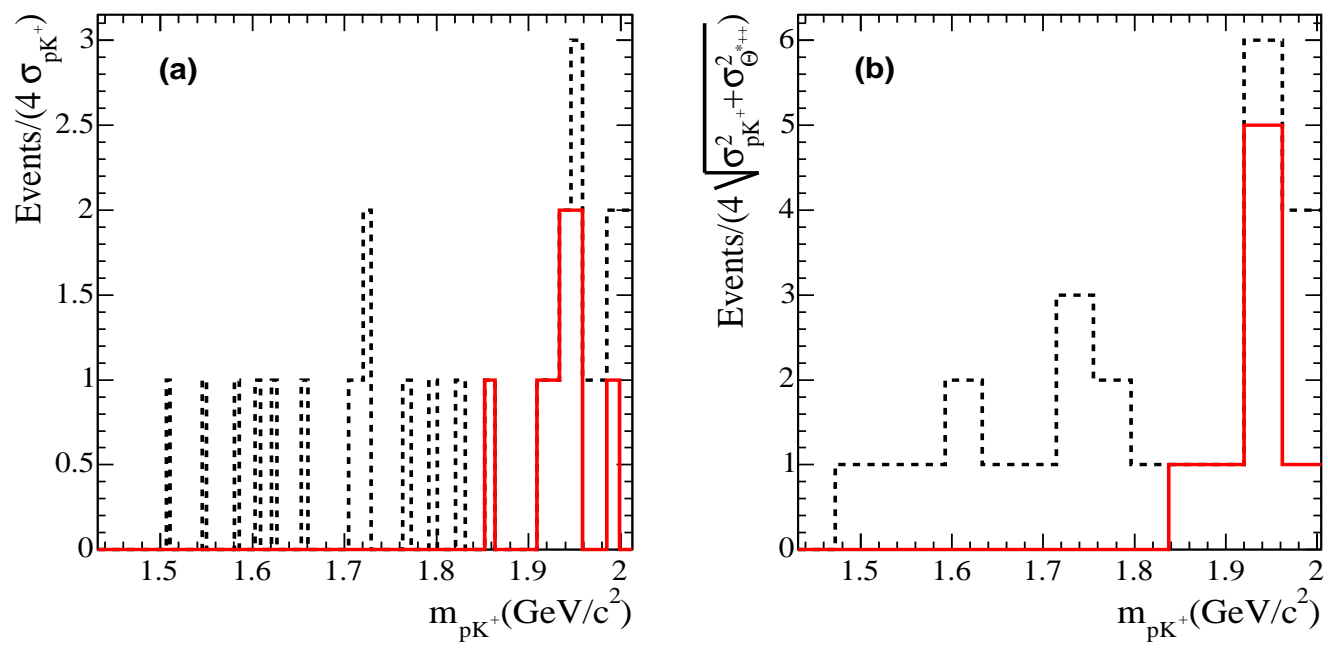

Figure 3.17: The $m_{p K^{+}}$distribution for data events in $B^{+} \rightarrow p \bar{p} K^{+}$signal box: events in the charmonium region $2.85<m_{p \bar{p}}<3.15 \mathrm{GeV} / \mathrm{c}^{2}$ (solid), events outside the charmonium region (dashed). (a) neglecting the width for $\Theta^{*++}$; (b) $\sigma_{\Theta^{*++}}=10 \mathrm{MeV}$. Note that these distributions are not efficiency-corrected.
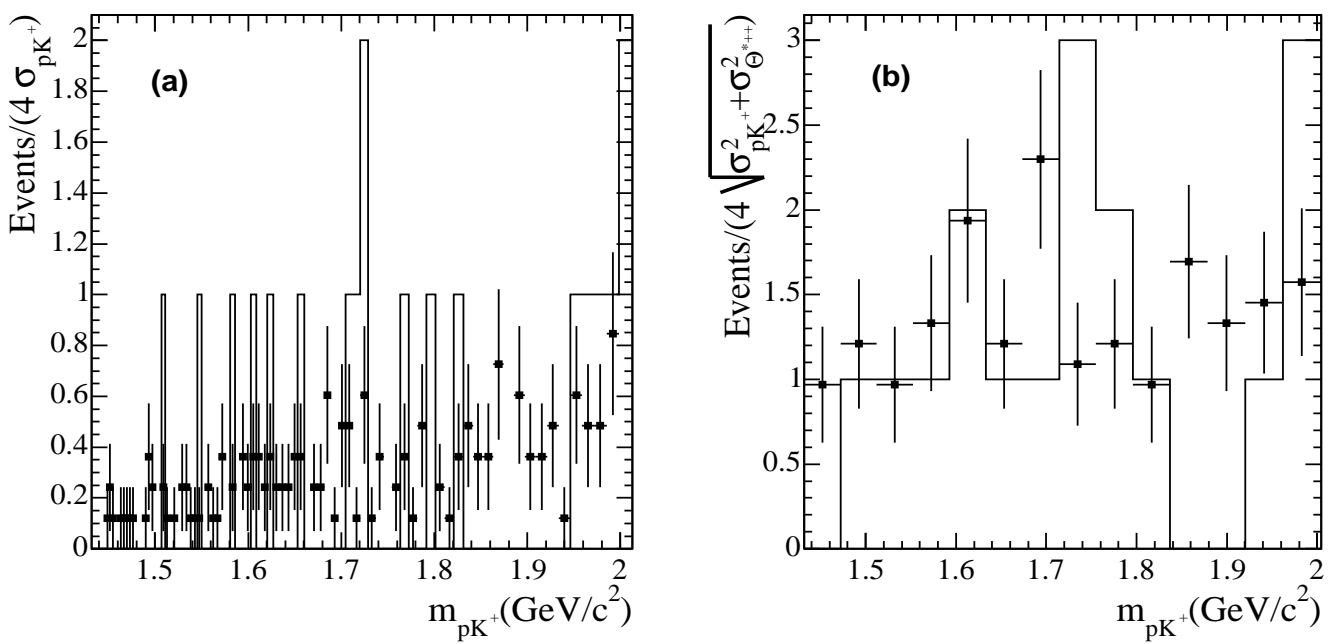

Figure 3.18: The $m_{p K^{+}}$distributions for data reconstructed as $B^{+} \rightarrow p \bar{p} K^{+}$(solid line) and rescaled $m_{E S}$ sideband (dots). (a) neglecting the width for $\Theta^{*++}$; (b) $\sigma_{\Theta^{*++}}=10 \mathrm{MeV}$. Note that these distributions are not efficiency-corrected. 

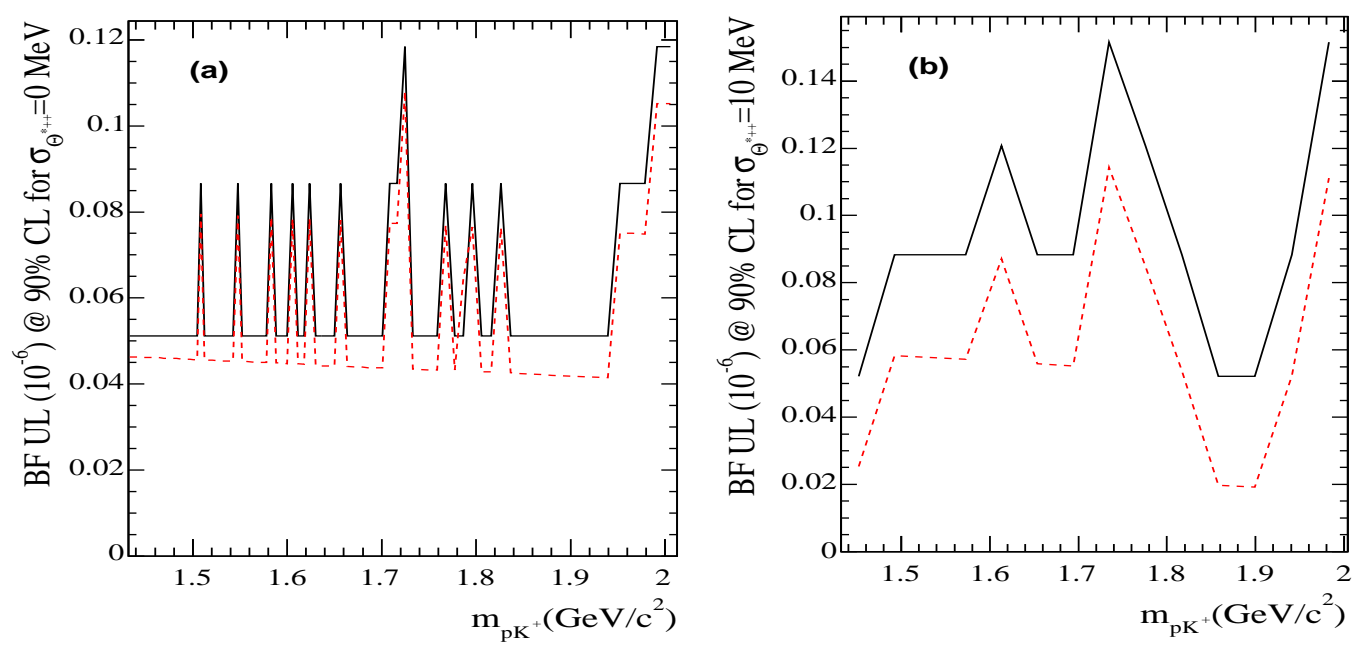

Figure 3.19: Upper Limit on the branching fraction of $B^{+} \rightarrow \Theta^{*++}\left(p K^{+}\right) \bar{p}$ at $90 \%$ confidence level with the assumption of no background(solid), with background as determined from $m_{E S}$ sideband data (dashed). (a) neglecting the width for $\Theta^{*++}$; (b) $\sigma_{\Theta^{*++}}=10 \mathrm{MeV}$. The systematic error correction is included in the limits.

of dedicated $B^{+} \rightarrow \Theta^{*++}\left(p K^{+}\right) \bar{p} \mathrm{MC}^{5}\left(\mathrm{MC}_{\Theta}\right)$ with $m_{\Theta^{*++}}=1.5,1.6,1.7,1.8,1.9$ $\mathrm{GeV} / \mathrm{c}^{2}$. The results of both Monte Carlo simulations are shown in Figure 3.16(a) and are consistent with each other. We will use the average $B^{+} \rightarrow \Theta^{*++}\left(p K^{+}\right) \bar{p}$ signal efficiency value obtained from $\mathrm{MC}_{\Theta}$ (as it has smaller errors), $(20.5 \pm 0.1) \%$ for $1.43<m_{p K^{+}}<2.00 \mathrm{GeV} / \mathrm{c}^{2}$, for this analysis.

In Figure 3.17 we separate the events into those inside the charmonium window and those outside. For the purpose of this search we remove the region of the main charmonium resonances $\eta_{c}$ and $J / \psi: 2.85<m_{p \bar{p}}<3.15 \mathrm{GeV} / \mathrm{c}^{2}$.

We search for $\Theta^{*++}$ pentaquark in the $p K^{+}$mass spectrum, shown in Figure 3.18 (charmonium resonances removed). The $m_{p K^{+}}$distribution of the combinatoric background shown in Figure 3.18 is obtained from the events in the data $m_{E S}$ sideband region and is scaled to the expected number of the combinatoric background events in the signal box.

\footnotetext{
${ }^{5}$ To generate these MC samples $\Delta^{++}$with different mass settings and width of $1 \mathrm{MeV}$ was used to represent $\Theta^{*++}$. All the $\Lambda_{c}$ decays into $\Delta^{++}$were set to zero.
} 
To set an upper limit on the branching fraction of $B^{+} \rightarrow \Theta^{*++}\left(p K^{+}\right) \bar{p}$ we count events in each of the $m_{p K^{+}}$mass bins in Figure 3.18 assuming that all the events observed are $B^{+} \rightarrow \Theta^{*++}\left(p K^{+}\right) \bar{p}$ signal events.

We use two methods to determine the upper limit. In the first one we assume that there is no background contribution. We calculate from Table 31.3 [20] the Bayesian upper limit at $90 \%$ confidence level as a function of $m_{p K^{+}}$assuming Poissondistributed events in the absence of background. The resulting values are shown in Figure 3.19. To account for systematic errors we increase the upper limit by the total systematic error $(6.1 \%)$.

To calculate the upper limit in the presence of background we use a tool described in [146]. It uses the toy Monte Carlo technique to calculate an upper limit in presence of uncertainties on the efficiency and the number of expected background events. We assume all the systematic errors except the systematics on background and $B$ counting to contribute to the uncertainty on the efficiency $(6.0 \%)$. To estimate the number of expected background events we fit a first-order polynomial to the $p K^{+}$mass spectrum of the combinatoric background events. The uncertainty on the background comes from the statistical error on the fit as well as the systematic error on the background. The resulting values of the upper limit as a function of $m_{p K^{+}}$increased by the systematic error on $B$-counting (1.1\%) are shown in Figure 3.19.

To simplify the presentation of the upper limit on the branching fraction as a function $m_{p K^{+}}$we assume that the number of events in each of the bins in $m_{p K^{+}}$is equal to the maximum number of events per bin for each of the $m_{p K^{+}}$regions (see Table 3.4). The resulting upper limit values for $\sigma_{\Theta^{*++}}=0 \mathrm{MeV}$ and $\sigma_{\Theta^{*++}}=10 \mathrm{MeV}$ are not significantly different.

\subsection{Systematic Studies}

The systematic uncertainties for each analysis are summarized in Table 3.5. The $\Upsilon(4 S)$ is assumed to decay equally to $B^{0} \bar{B}^{0}$ and $B^{+} B^{-}$mesons. Incomplete knowledge of the luminosity and cross-section leads to a $1.1 \%$ uncertainty [147]. Chargedtracking and particle-identification (PID) studies in the data lead to small corrections 
Table 3.4: Upper limits for the branching fraction of $B^{+} \rightarrow \Theta^{*++}\left(p K^{+}\right) \bar{p}$ as a function of $m_{p K^{+}}$without (with) background subtraction.

\begin{tabular}{||c|c|c|c||}
\hline \hline $\begin{array}{c}\text { Mass Region, } \\
\mathrm{GeV} / \mathrm{c}^{2}\end{array}$ & $\begin{array}{c}\text { Max Events } \\
(0 / 10 \mathrm{MeV}) \\
\text { in any } m_{p K^{+}} \text {bin }\end{array}$ & $\begin{array}{c}\text { BF UL }\left(10^{-7}\right) \\
\text { @ 90\% CL } \\
\text { for } \sigma_{\Theta^{*++}}=0 \mathrm{MeV}\end{array}$ & $\begin{array}{c}\text { BF UL }\left(10^{-7}\right) \\
\text { @ 90\% CL } \\
\text { for } \sigma_{\Theta^{*++}}=10 M e V\end{array}$ \\
\hline $1.4<m_{p K^{+}}<1.5$ & $0 / 0$ & $0.52(0.47)$ & $0.52(0.25)$ \\
$1.5<m_{p K^{+}}<1.6$ & $1 / 1$ & $0.87(0.80)$ & $0.87(0.58)$ \\
$1.6<m_{p K^{+}}<1.7$ & $1 / 2$ & $0.87(0.80)$ & $1.19(0.87)$ \\
$1.7<m_{p K^{+}}<2.0$ & $2 / 3$ & $1.19(1.08)$ & $1.49(1.14)$ \\
\hline \hline
\end{tabular}

Table 3.5: Systematic uncertainties in percent on the branching fraction measurements and in the values of uncertainties for the asymmetry measurements. Values for $m_{p \bar{p}}$ below $2.85 \mathrm{GeV} / c^{2}$ are given in parenthesis.

\begin{tabular}{|l|c|c|c|c|c|}
\hline Type & $p \bar{p} K^{+}$ & $\eta_{c} K^{+}$ & $p \Lambda(1520)$ & $\bar{p} \Theta^{*++}$ & $A_{c h}$ \\
\hline B-counting & $1.1(1.1)$ & 1.1 & 1.1 & 1.1 & - \\
Tracking/PID & $3.8(3.8)$ & 3.4 & 4.2 & 4.2 & 0.02 \\
MC Statistics & $2.1(2.4)$ & 0.7 & 1.0 & 0.5 & 0.03 \\
B.F. Errors & $0.9(-)$ & - & 2.2 & - & - \\
Selection & $0.2(-)$ & 0.4 & 3.9 & 3.9 & - \\
$\Delta E /$ Mass Fits & $3.6(2.4)$ & 8.9 & 14.3 & - & 0.01 \\
\hline Total & $5.8(5.2)$ & 13.5 & 15.6 & 6.1 & 0.03 \\
\hline
\end{tabular}


applied to each track in these simulations. Limitation of statistics and purity in these data-MC comparisons lead to residual tracking/PID uncertainties. A large control sample of $B^{+} \rightarrow J / \psi\left(e^{+} e^{-}\right) K^{+}$is separately studied in data and MC simulations to understand the residual errors from the event-shape, $\Delta E$, and $m_{E S}$ cuts. Limitation of $\mathrm{MC}$ statistics employed in each analysis contributes to a small uncertainty. Branching fraction uncertainties (B.F. Errors) [20] on $\mathcal{B}\left(B^{+} \rightarrow X K^{+}\right) \times \mathcal{B}(X \rightarrow p \bar{p})$, where $X=\chi_{c[0,1,2]}, \psi^{\prime}$ and $\mathcal{B}\left(\Lambda(1520) \rightarrow p K^{-}\right)$affect the total $p \bar{p} K^{+}$and the $p \bar{\Lambda}$ branching fraction measurements, respectively. Where the $\mathrm{MC}$ values are used to fix signal shape parameters in a fit, the parameters are varied within their uncertainties and the data are refit to propagate this uncertainty. In a similar fashion, different ranges and background functions are employed to establish the uncertainty on the mass spectra fits (resulting, for example, in the $\Gamma\left(\eta_{c}\right)$ uncertainty of $3 \mathrm{MeV}$ ).

\subsection{Summary}

In summary, with $210 \mathrm{fb}^{-1}$ of data, we isolate the $B^{+} \rightarrow p \bar{p} K^{+}$final state, and measure its non-charmonium branching fraction to be $(5.3 \pm 0.4 \pm 0.3) \times 10^{-6}$ for $m_{p \bar{p}}$ below $2.85 \mathrm{GeV} / c^{2}$ and $(6.7 \pm 0.5 \pm 0.4) \times 10^{-6}$ for the whole $m_{p \bar{p}}$ range. We measure $A_{c h}=-0.16_{-0.08}^{+0.07} \pm 0.04$ for $m_{p \bar{p}}$ below $2.85 \mathrm{GeV} / c^{2}$. We measure the total width of $\eta_{c}$ to be $25_{-5}^{+6} \pm 3 \mathrm{MeV} / c^{2}$. An upper limit of the decay rate to $p \bar{\Lambda}(1520)$ is set at $1.5 \times 10^{-6}$. No evidence is found for the pentaquark candidate $\Theta^{*++}$ in the mass range 1.43 to $2.0 \mathrm{GeV} / c^{2}$, decaying into $p K^{+}$, and branching fraction limits are established at the $10^{-7}$ level. 


\section{Chapter 4}

\section{The $B \rightarrow p \bar{p} h$ Maximum Likelihood Analysis}

In this chapter we address the rest of processes $B \rightarrow p \bar{p} h\left(h=K^{+}, K_{S}^{0}, K^{*+}, K^{* 0}, \pi^{+}\right)$. Instead of applying the analysis method described in the previous chapter, we use a different method which relies on the Maximum Likelihood (ML) fitting in at least three variables and sPlots for the mass projection studies. These techniques are suited for the study of these channels, because they have either lower branching fractions or lower reconstruction efficiency.

\subsection{Event Selection}

\subsubsection{Track reconstruction}

The track requirements are divided between two categories: one for "prompt" tracks which are produced at the $B$ decay vertex (see Section 3.1), and one for secondary tracks from $K_{S}^{0}$ decays. The criteria for the $K_{S}^{0} \rightarrow \pi^{+} \pi^{-}$tracks are loosened compared to "prompt" tracks; tracks are not required to come from the interaction point, nor to have a minimum $p_{t}$ or a minimum number of DCH hits. 


\subsubsection{Particle Identification}

Particle identification used in this analysis is identical to that of $B^{+} \rightarrow p \bar{p} K^{+}$branching fraction measurement as described in detail in Section 3.2. The pions in the $B^{+} \rightarrow$ $p \bar{p} \pi^{+}$mode are required not to pass kaon identification criteria as well as not to pass proton, electron or muon selection. There are no PID requirements on the pions coming from the $K^{0 *} \rightarrow K^{+} \pi^{-}$or $K^{*+} \rightarrow K^{0} \pi^{+}$.

\subsection{3 $K_{S}^{0}$ Selection}

Candidate $K_{S}^{0}$ 's are reconstructed in $K_{S}^{0} \rightarrow \pi^{+} \pi^{-}$mode. A pair of oppositely charged tracks are fit to a common vertex using the helical track parameters at the origin using the so-called Cascade vertex fitter [148]. The Cascade fitter algorithm calculates the point of closest approach of the two tracks based on their trajectories and determines the most likely point of origin by minimizing the $\chi^{2}$ based on the track covariance matrix. From its lifetime $\left(\tau_{K_{S}^{0}}=0.894 \times 10^{10} \mathrm{~s}, c \tau=2.68 \mathrm{~cm}[20]\right)$, the $K_{S}^{0}$ is expected to traverse an appreciable distance from the production point before decaying. As a result, the track parameters may be significantly different at the decay vertex from those obtained at the origin. The track momenta are reevaluated at the calculated vertex, resulting in a significant improvement in the mass resolution.

To select events for the maximum likelihood fit the following the $K_{S}^{0}$ quality cuts are applied (Figure 4.1):

- $\cos \alpha_{K_{S}^{0}}>0.999$

- $K_{S}^{0}$ vertex probability $>10^{-4}$

- $\left|m_{K_{S}^{0}}^{P D G}-m_{K_{S}^{0}}^{r e c o}\right|=\left|\Delta m_{K_{S}^{0}}\right|<8 \mathrm{MeV} / c^{2}$ or $3 \sigma$ of $K_{S}^{0}$ mass resolution

where $\alpha_{K_{S}^{0}}$ is the angle between the $K_{S}^{0}$ flight direction and a line connecting the $B$ and the $K_{S}^{0}$ vertices. 

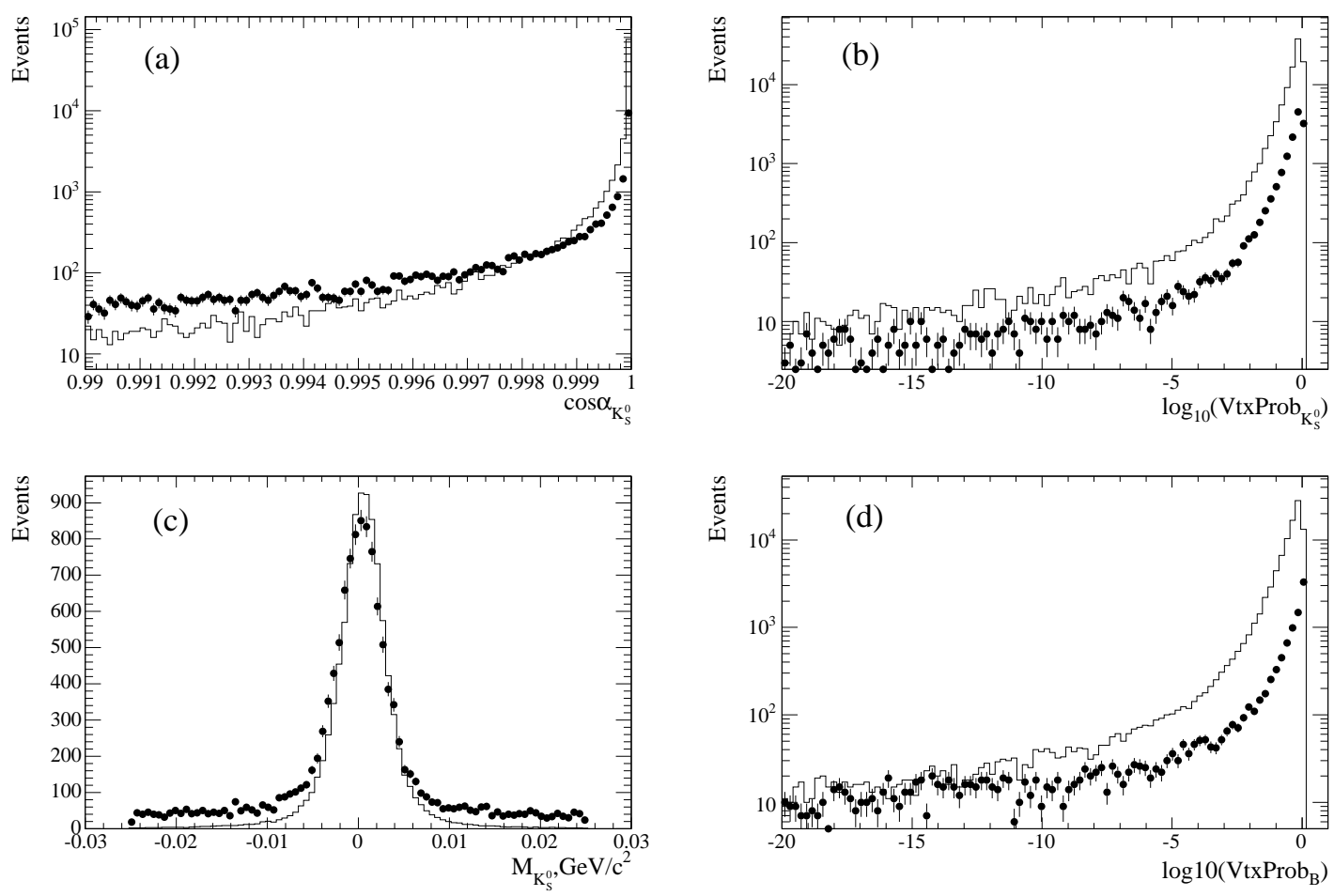

Figure 4.1: Relevant distributions of discriminating variables: (a) $\cos \alpha_{K_{S}^{0}}$, (b) $K_{S}^{0}$ vertex probability, (c) $K_{S}^{0}$ mass and (d) $B$ vertex probability. Solid histogram - signal MC. Points - off-peak data. 


\subsection{4 $K^{*+}$ Selection}

Candidate $K^{*+}$ 's are reconstructed in the $K^{* \pm} \rightarrow K_{S}^{0} \pi^{ \pm}$mode which constitutes $66.7 \%$ [20] of possible $K^{*+}$ decays. It is reconstructed by vertexing $K_{S}^{0}$ with a "prompt" charged track. The candidates whose mass is within $80 \mathrm{MeV} / c^{2}$ of the nominal $K^{*+}$ mass $\left(891.7 \mathrm{MeV} / c^{2}\right)$ are selected as $K^{*+}$ candidates, and the ones with mass $160-240 \mathrm{MeV} / c^{2}$ away from the $K^{*+}$ mass are chosen as the sideband (SB) control sample.

\subsection{5 $\quad K^{* 0}$ Selection}

Candidate $K^{* 0}$ S are reconstructed in the $K^{* 0} \rightarrow K^{ \pm} \pi^{\mp}$ mode which constitutes $66.7 \%$ [20] of possible $K^{* 0}$ decays. It is reconstructed by vertexing oppositely charged pairs of tracks, one of which has been identified as a kaon and assigned the kaon mass hypothesis. The candidates whose mass is within $80 \mathrm{MeV} / c^{2}$ of the nominal $K^{* 0}$ mass $\left(896.1 \mathrm{MeV} / c^{2}\right)$ are selected as $K^{* 0}$ candidates, and the ones with mass $160-240 \mathrm{MeV} / c^{2}$ away from the $K^{* 0}$ mass are chosen as the sideband (SB) control sample.

\subsubsection{B Selection}

The $B$ candidate is formed by vertexing the proton, the anti-proton and the $h$ candidates using the Cascade vertex fitter. The $B$ meson vertex probability must be greater than $10^{-4} . \Delta E$ and $m_{E S}$ are used to isolate the $B \rightarrow p \bar{p} h$ signal taking advantage of the kinematic constraints of $B$ mesons produced at the $\Upsilon(4 S)$. The initial selection requires $|\Delta E|<0.35 \mathrm{GeV}$ and $m_{E S}>5.2 \mathrm{GeV}$.

\subsubsection{Background Characterization}

\section{Continuum $(q \bar{q})$ Background}

The Fisher discriminant described in Section 3.4.1 is used to provide discrimination between the large continuum background $\left(e^{+} e^{-} \rightarrow q \bar{q}\right.$, where $\left.q=u, d, s, c\right)$, which tends to be collimated along the original quark direction, and more spherical $B \bar{B}$ 
events. The Fisher discriminant coefficients are optimized for each of the modes separately using the signal distributions obtained from $B \rightarrow p \bar{p} h$ simulated events that are distributed uniformly in phase-space $(B \rightarrow p \bar{p} h$ signal Monte Carlos) and the background shape from off-peak data.

\section{$B$ Background}

For $B \rightarrow p \bar{p} h$ analysis the main source of the $B \bar{B}$ backgrounds decaying into the same final states as the signal are the $b \rightarrow c \bar{c} s$ transitions, where $B \rightarrow X_{c \bar{c}} K, X_{c \bar{c}} \rightarrow p \bar{p}$ and $X_{c \bar{c}}=\eta_{c}, J / \psi, \psi(2 S), \chi_{c 0,1,2}$ (charmonium background) shown in Table 4.1. This background is comparable in size to signal. To check for additional $B \bar{B}$ backgrounds that might peak in the ML fit region (as defined in Table 4.3), we study generic $B \bar{B}$ Monte Carlo as well as a set of samples of exclusive $B$ decay simulated events for potential charmoniumless backgrounds. The expected $B \bar{B}$ backgrounds are primarily from those modes with charmed baryons (in particular $\Lambda_{c}^{+}$) decaying into the same final states as the signal. They are shown in Table 4.2. Other "charmoniumless" and charmless modes are negligible or their distribution in $m_{E S}-\Delta E$ plane is indistinguishable from that of continuum data.

\subsubsection{Summary of the event selection.}

The final event selection criteria are summarized in Table 4.3. After the selection described above some signal Monte Carlo events have more than one $B$ candidates. To select a single candidate per event for further analysis, we keep candidate with the largest product of $K_{S}^{0}$ and $B$ vertexing probabilities for the modes which have $K_{S}^{0}$ or with the largest $B$ vertexing probability for the modes without $K_{S}^{0}$. The resulting percentage of signal Monte-Carlo events with one or more tracks mis-reconstructed, so-called self-cross-feed (SCF), is summarized in Table 4.3.

After selecting the $B$ candidates in the $\Delta E: m_{E S}$ region, a kinematic fit is performed for each $B$ candidate, fixing its mass and energy to their known values to be able to study the corresponding mass projections with improved resolution. 
Table 4.1: The expected rates for the peaking background events of the type $B^{0} \rightarrow$ $X h, X \rightarrow p \bar{p}$ and $h=K^{0}, K^{*+}, K^{* 0}, \pi^{+}$. Values in italic are taken from Ref. [149] and all others from Ref. [20]. To obtain the expected yields $K^{0}$ and $K^{*+}$ partial branching have to be multiplied by 0.5 for the number of $K^{0}$ which are $K_{S}^{0}$ and by $0.6894 \pm 0.0014$ for $K_{S}^{0} \rightarrow \pi^{+} \pi^{-} ; K^{*+}$ b.f. has to be multiplied by 0.667 for $K^{*+} \rightarrow K^{0} \pi^{+}$and $K^{* 0}$ b.f. has to be multiplied by 0.667 for $K^{* 0} \rightarrow K^{+} \pi^{-}$.

\begin{tabular}{||c|c|c|c|c|c|c|}
\hline \hline$X / K^{0}$ & $M_{X}$ & $\mathcal{B}_{X \rightarrow p \bar{p}} \cdot 10^{-3}$ & $\mathcal{B}_{B \rightarrow X K^{0} \cdot 10^{-3}}$ & $\mathcal{B}_{\text {part }} \cdot 10^{-6}$ & $\epsilon$ & events \\
\hline$\eta_{c}$ & 2980 & $1.3 \pm 0.4$ & $1.2 \pm 0.4$ & $1.56 \pm 0.71$ & 35.8 & 46.7 \\
\hline$J / \psi$ & 3097 & $2.12 \pm 0.10$ & $0.85 \pm 0.04$ & $1.80 \pm 0.08$ & 36.6 & 53.8 \\
\hline$\chi_{c 0}(1 P)$ & 3415 & $0.22 \pm 0.03$ & $<0.5$ & $<0.13$ & 36.0 & $<3.6$ \\
\hline$\chi_{c 1}(1 P)$ & 3511 & $0.072 \pm 0.013$ & $0.40 \pm 0.12$ & $0.03 \pm 0.01$ & 36.0 & 0.8 \\
\hline$\chi_{c 2}(1 P)$ & 3556 & $0.068 \pm 0.007$ & $<0.041$ & $<0.003$ & 36.0 & $<0.08$ \\
\hline$\psi(2 S)$ & 3686 & $0.207 \pm 0.031$ & $0.62 \pm 0.07$ & $0.13 \pm 0.02$ & 36.0 & 3.7 \\
\hline \hline$X / K^{*+}$ & $M_{X}$ & $\mathcal{B}_{X \rightarrow p \bar{p} \cdot 10^{-3}}$ & $\mathcal{B}_{B \rightarrow X K^{*+} \cdot 10^{-3}}$ & $\mathcal{B}_{\text {part }} \cdot 10^{-6}$ & $\epsilon$ & events \\
\hline$\eta_{c}$ & 2980 & $1.3 \pm 0.4$ & & & 17.6 & \\
\hline$J / \psi$ & 3097 & $2.12 \pm 0.10$ & $1.35 \pm 0.010$ & $2.86 \pm 0.25$ & 19.2 & 42.5 \\
\hline$\chi_{c 0}(1 P)$ & 3415 & $0.22 \pm 0.03$ & & & & \\
\hline$\chi_{c 1}(1 P)$ & 3511 & $0.072 \pm 0.013$ & $0.294 \pm 0.137$ & $0.02 \pm 0.01$ & 19 & 0.31 \\
\hline$\chi_{c 2}(1 P)$ & 3556 & $0.068 \pm 0.007$ & $<0.012$ & $<0.001$ & & \\
\hline$\psi(2 S)$ & 3686 & $0.207 \pm 0.031$ & $0.70^{\prime} \pm 0.085$ & $0.15 \pm 0.04$ & 18 & 2.7 \\
\hline \hline$X / K^{* 0}$ & $M_{X}$ & $\mathcal{B}_{X \rightarrow p \bar{p} \cdot 10^{-3}}$ & $\mathcal{B}_{B \rightarrow X K^{* 0} \cdot 10^{-3}}$ & $\mathcal{B}_{\text {part }} \cdot 10^{-6}$ & $\epsilon$ & events \\
\hline$\eta_{c}$ & 2980 & $1.3 \pm 0.4$ & $1.6 \pm 0.7$ & $2.08 \pm 1.11$ & 23.7 & 66.7 \\
\hline$J / \psi$ & 3097 & $2.12 \pm 0.10$ & $1.31 \pm 0.07$ & $2.78 \pm 0.20$ & 25.0 & 107.5 \\
\hline$\chi_{c 0}(1 P)$ & 3415 & $0.22 \pm 0.03$ & $<0.77$ & $<0.17$ & 25.7 & 6.8 \\
\hline$\chi_{c 1}(1 P)$ & 3511 & $0.072 \pm 0.013$ & $0.32^{r} 7 \pm 0.076$ & $0.024 \pm 0.007$ & 25.7 & 1.0 \\
\hline$\chi_{c 2}(1 P)$ & 3556 & $0.068 \pm 0.007$ & $<0.036$ & $<0.002$ & 25.7 & 0.08 \\
\hline$\psi(2 S)$ & 3686 & $0.207 \pm 0.031$ & $0.711 \pm 0.062$ & $0.147 \pm 0.026$ & 25.7 & 8.8 \\
\hline \hline$X / \pi^{+}$ & $M_{X}$ & $\mathcal{B}_{X \rightarrow p \bar{p}} \cdot 10^{-3}$ & $\mathcal{B}_{B \rightarrow X \pi^{+} \cdot 10^{-3}}$ & $\mathcal{B}_{\text {part }} \cdot 10^{-6}$ & $\epsilon$ & events \\
\hline$J / \psi$ & 3097 & $2.12 \pm 0.10$ & $0.048 \pm 0.004$ & $0.102 \pm 0.010$ & 50 & 11.8 \\
\hline$\chi_{c 0}(1 P)$ & 3415 & $0.22 \pm 0.03$ & $<0.061$ & $<0.013$ & & \\
\hline
\end{tabular}


Table 4.2: Estimated non-charmonium $B$ background events with $p \bar{p} h$ signal final states in $210 \mathrm{fb}^{-1}$ of data.

\begin{tabular}{||c|c|c|c|c|c|}
\hline Mode & $\mathcal{B}_{\Lambda_{c}^{+} \rightarrow p h} \cdot 10^{-3}$ & $\mathcal{B}_{B \rightarrow \Lambda_{c}^{+} \bar{p}} \cdot 10^{-3}$ & $\mathcal{B}_{\text {part }} \cdot 10^{-6}$ & $\epsilon$ & events \\
\hline$h=K^{0}$ & $23 \pm 6$ & $0.022 \pm 0.008$ & $0.51 \pm 0.23$ & 30.0 & 12 \\
\hline \hline$h=K^{* 0}$ & $16 \pm 5$ & $0.022 \pm 0.008$ & $0.35 \pm 0.17$ & 22.5 & 12.7 \\
\hline \hline & $\mathcal{B}_{\Lambda_{c}^{+} \rightarrow p K^{0}} \cdot 10^{-3}$ & $\mathcal{B}_{B \rightarrow \Lambda_{c}^{+} \bar{p}} \cdot 10^{-3}$ & $\mathcal{B}_{\text {part }} \cdot 10^{-6}$ & $\epsilon$ & events \\
\hline$K^{*+}$ & $23 \pm 6$ & $0.201 \pm 0.025$ & $4.62 \pm 1.30$ & 0.05 & 0.2 \\
\hline \hline
\end{tabular}

Table 4.3: Summary of the event selections (" $\sqrt{ }$ means "required").

\begin{tabular}{||c||c|c|c|c|c||}
\hline Cut/Mode & $p \bar{p} K^{+}$ & $p \bar{p} K^{0}$ & $p \bar{p} K^{*+}$ & $p \bar{p} K^{* 0}$ & $p \bar{p} \pi^{+}$ \\
\hline Proton ID & $\sqrt{ }$ & $\sqrt{ }$ & $\sqrt{ }$ & $\sqrt{ }$ & $\sqrt{ }$ \\
Kaon ID & $\sqrt{ }$ & - & - & $\sqrt{ }$ & - \\
Pion ID & - & - & - & - & $\sqrt{ }$ \\
$m_{\mathrm{ES}}>\left(\mathrm{GeV} / c^{2}\right)$ & 5.22 & 5.22 & 5.22 & 5.22 & 5.25 \\
$|\Delta E|<(\mathrm{GeV})$ & 0.15 & 0.15 & 0.10 & 0.10 & 0.10 \\
$B$ vtx prob $>$ & $10^{-4}$ & $10^{-4}$ & $10^{-4}$ & $10^{-4}$ & $10^{-4}$ \\
$K_{S}$ vtx prob $>$ & - & $10^{-4}$ & $10^{-4}$ & - & - \\
$\left|\Delta m_{K_{S}^{0}}\right|<\left(\mathrm{MeV} / c^{2}\right)$ & - & 8 & 8 & - & - \\
$\cos \alpha_{K_{S}^{0}}>$ & - & 0.999 & 0.999 & - & - \\
$\left|\Delta m_{K^{*}}\right|<\left(\mathrm{MeV} / c^{2}\right)$ & - & - & 80 & 80 & - \\
\hline$\%$ of self-cross-feed & 3.0 & 0.5 & 5.6 & 4.3 & 1.6 \\
\hline \multicolumn{2}{|r|}{}
\end{tabular}


Table 4.4: Maximum Likelihood Fit Event Categories (" $\sqrt{ }$ " means that this event category is used in one of the fit regions of the mode).

\begin{tabular}{||c||c|c|c|c|c||}
\hline Categories & $p \bar{p} K^{+}$ & $p \bar{p} K^{0}$ & $p \bar{p} K^{*+}$ & $p \bar{p} K^{* 0}$ & $p \bar{p} \pi^{+}$ \\
\hline signal & $\sqrt{ }$ & $\sqrt{ }$ & $\sqrt{ }$ & $\sqrt{ }$ & $\sqrt{ }$ \\
SCF & $\sqrt{ }$ & - & $\sqrt{ }$ & $\sqrt{ }$ & $\sqrt{ }$ \\
combinatorial & $\sqrt{ }$ & $\sqrt{ }$ & $\sqrt{ }$ & $\sqrt{ }$ & $\sqrt{ }$ \\
$J / \psi$ & $\sqrt{ }$ & $\sqrt{ }$ & $\sqrt{ }$ & $\sqrt{ }$ & - \\
$\eta_{c}$ & $\sqrt{ }$ & $\sqrt{ }$ & $\sqrt{ }$ & $\sqrt{ }$ & - \\
$\Lambda_{c}^{+}$ & $\sqrt{ }$ & $\sqrt{ }$ & $\sqrt{ }$ & $\sqrt{ }$ & - \\
$p \bar{p} K^{+}$ & - & - & - & - & $\sqrt{ }$ \\
$p \bar{p} K^{+}$SCF & - & - & - & - & $\sqrt{ }$ \\
\hline
\end{tabular}

\subsection{Maximum Likelihood Fit}

Yields for signal and background events are determined using an extended maximum likelihood (ML) fit. The extended likelihood is given by

$$
\mathcal{L}=\exp \left(-\sum_{k} n_{k}\right) \prod_{j=1}^{N}\left(\sum_{k} n_{k} P_{k}(\vec{\alpha}, \vec{x})\right)_{j}
$$

where $P_{k}(\vec{\alpha}, \vec{x})$ is the product of probability density functions (PDFs) for event category $k$ (see Table 4.4 ) with PDF parameters $\vec{\alpha}$ and event variables $\vec{x}$ for event $j$ out of $N$ events entering the fit. The yields are represented by the parameters $n_{k}$ and are the free parameters in the fit.

Extraction of the non-resonant $B \rightarrow p \bar{p} h$ event yield is made more complicated by the potential presence of the resonances containing a charm quark decaying into $p \bar{p} h$ final state. The main resonances, such as $\Lambda_{c}^{+}$or the charmonium resonances, are shown in Figure 4.2 for the $B^{0} \rightarrow p \bar{p} K_{S}^{0}$ mode. To extract non-resonant (charmless) signal, the amount of charm and charmonium resonant contributions are determined by fitting either $p \bar{p}$ or $p h$ mass spectra. The fit mass region is chosen in such a way that the non-resonant signal is expected to be approximately flat (more so if the mass 


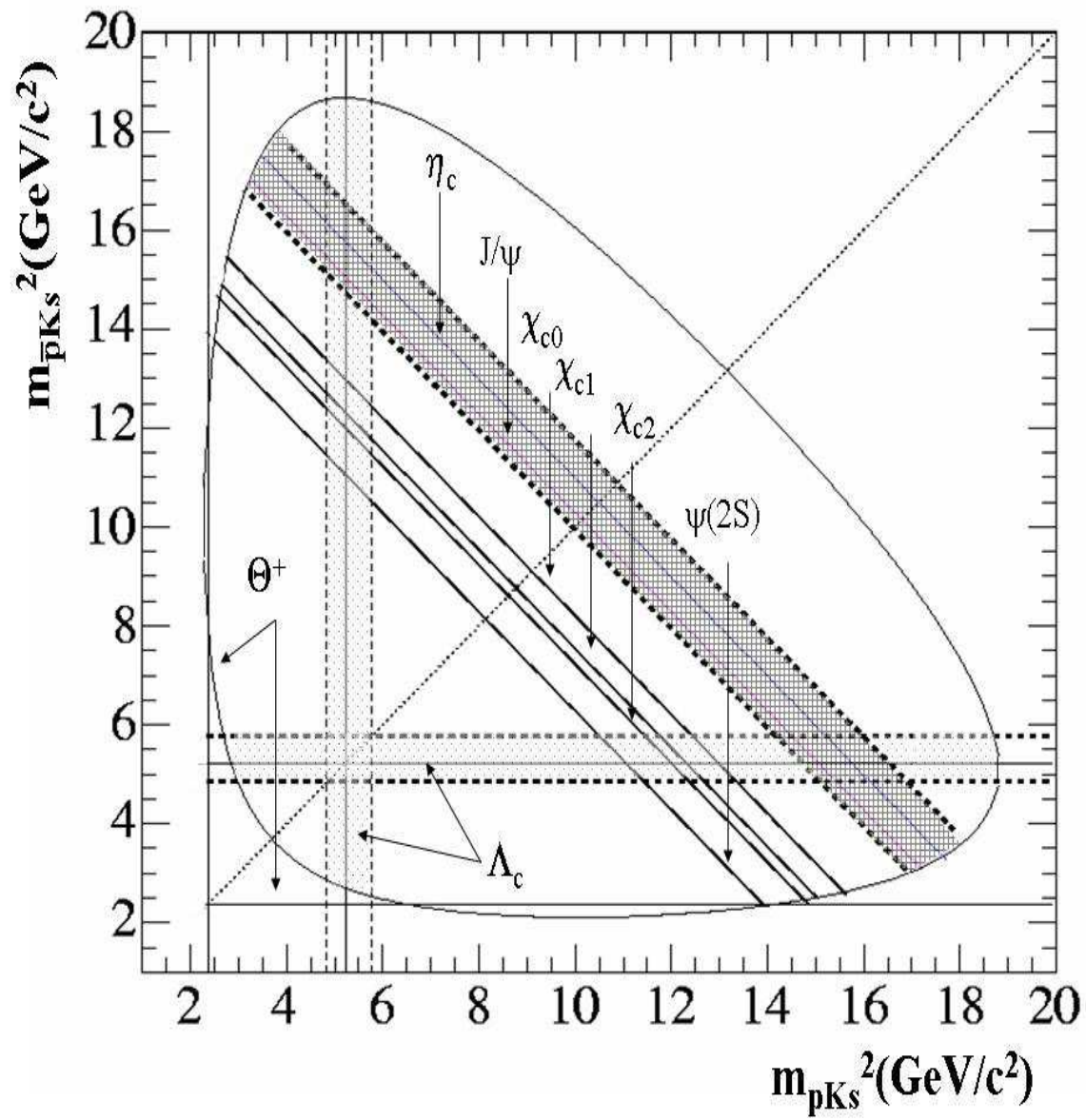

Figure 4.2: $B^{0} \rightarrow p \bar{p} K_{S}^{0}$ Dalitz plot with bands of sub-resonance structures. 
Table 4.5: The total number of event categories $\left(N_{s}\right)$ in Maximum Likelihood Fit Regions.

\begin{tabular}{||c||c|c|c|c|c||}
\hline Region & $p \bar{p} K^{+}$ & $p \bar{p} K^{0}$ & $p \bar{p} K^{*+}$ & $p \bar{p} K^{* 0}$ & $p \bar{p} \pi^{+}$ \\
\hline charmonium & 5 & 4 & 5 & 5 & - \\
charm & - & 3 & - & 4 & - \\
all-other & 3 & 2 & 3 & 3 & 5 \\
\hline
\end{tabular}

Table 4.6: Maximum Likelihood Fit Variables (" $\sqrt{ }$ " means "used").

\begin{tabular}{||c||c|c|c|c||}
\hline \multicolumn{1}{||c||}{$\begin{array}{c}\text { Event } \\
\text { Variables }\end{array}$} & \multicolumn{2}{c||}{ Branching Fraction Calculation } & \multicolumn{2}{c||}{ Resonant } \\
charmonium & charm & all-other & Structure \\
\hline$m_{\mathrm{ES}}$ & $\sqrt{ }$ & $\sqrt{ }$ & $\sqrt{ }$ & $\sqrt{ }$ \\
$\Delta E$ & $\sqrt{ }$ & $\sqrt{ }$ & $\sqrt{ }$ & $\sqrt{ }$ \\
$\mathcal{F}$ & $\sqrt{ }$ & $\sqrt{ }$ & $\sqrt{ }$ & $\sqrt{ }$ \\
$m_{p \bar{p}}$ & $\sqrt{ }$ & - & - & - \\
$m_{p h}$ & - & $\sqrt{ }$ & - & - \\
\hline
\end{tabular}

region is taken to be fairly narrow) while the resonance has a Gaussian or Voigtian ${ }^{1}$ distribution. Three main regions of interest in this analysis are shown in Figure 4.2 and summarized in Table 4.5:

- the "main" charmonium region with includes $\eta_{c}$ and $J / \psi$ resonances: $2.85<m_{p \bar{p}}$ $<3.15 \mathrm{MeV} / \mathrm{c}^{2}$ (for $K^{+} / K^{0} / K^{* 0} / K^{*+}$ modes). Event categories: signal, selfcross-feed, $J / \psi, \eta_{c}$, combinatorial.

- the $\Lambda_{c}^{+}$charm region(two possible projections are summed): $\left|m_{p \bar{p}}-3\right|>0.15 \mathrm{GeV} / \mathrm{c}^{2}$ and $\left|m_{p h_{S}}-2.3\right|<0.1 \mathrm{GeV} / \mathrm{c}^{2}$ (for $K^{0} / K^{* 0}$ modes). Event categories: signal, self-cross-feed, $\Lambda_{c}^{+}$, combinatorial.

- the "all-other" region. Event categories: signal, self-cross-feed, combinatorial $\left(p \bar{p} K^{+}\right.$and its self-cross-feed for $B^{+} \rightarrow p \bar{p} \pi^{+}$mode).

\footnotetext{
${ }^{1} \mathrm{~A}$ convolution of a Gaussian and a Breit-Wigner distributions.
} 
The event variables $\vec{x}$ used for the fit in each of the regions are summarized in Table 4.6. Note that for the detailed study of the resonant structures the threevariable $\left(m_{\mathrm{ES}}, \Delta E\right.$ and $\left.\mathcal{F}\right)$ maximum likelihood fit is performed in the whole Dalitz plot simultaneously. The PDFs used to model these as well as PDF parameters $\vec{\alpha}$ are determined from data or Monte Carlo samples and are described in detail in the next section.

The sPlot technique is used to extract the branching fraction from the fit yields [150, 151]. By using the PDF information to assign a "signal weight" to each event, this method correctly deals with the difficulty that the signal efficiency is not necessarily constant over the Dalitz plot. In this method, for example, one calculates the branching fraction $B^{0} \rightarrow p \bar{p} K_{S}^{0}$ from the fit yields as:

$$
\mathcal{B}=\frac{\sum_{j=1}^{N} \frac{\left(\mathcal{P}_{\text {signal }}\right)_{j}}{\varepsilon_{j}}}{N_{B \bar{B}} \cdot \mathcal{B}\left(K_{S}^{0} \rightarrow \pi^{+} \pi^{-}\right)},
$$

where

$$
\left(\mathcal{P}_{\text {signal }}\right)_{j}=\frac{\sum_{l=1}^{N_{s}} \mathbf{V}_{\text {signal }, l} P_{l}\left(\vec{\alpha}, \vec{x}_{j}\right)}{\sum_{k=1}^{N_{s}} n_{k} P_{k}\left(\vec{\alpha}, \vec{x}_{j}\right)} .
$$

As above, $P$ is the PDF for an event category $k, l$ where $k, l$ are described in Table 4.4 and $N_{s}$ is total number of event categories. $\vec{x}_{j}$ are the event variables (see Table 4.6) for event $j$ from the total events $N$ in the fit which returns the yields $n_{k}$ and the covariance matrix $\mathbf{V} . N_{B \bar{B}}$ is the number of $B \bar{B}$ pairs in the dataset and $\varepsilon_{j}$ is the signal efficiency as a function of each event's Dalitz plot location.

The statistical error on branching fraction is calculated using the following equation:

$$
\sigma(\mathcal{B})=\frac{\sqrt{\sum_{j=1}^{N} \frac{\left(\mathcal{P}_{\text {signal }}\right)_{j}^{2}}{\varepsilon_{j}^{2}}}}{N_{B \bar{B}} \cdot \mathcal{B}\left(K_{S}^{0} \rightarrow \pi^{+} \pi^{-}\right)} .
$$

The event yields $n_{k}$ are floated in the fit in order to maximize the likelihood $\mathcal{L}$. The corresponding $\chi^{2}$ is defined as

$$
\chi^{2}=-2 \cdot \ln \left(\mathcal{L} / \mathcal{L}_{\max }\right),
$$


where $\mathcal{L}_{\text {max }}$ is the maximum likelihood value. The statistical significance $\sigma$ of the fitted signal $n_{\text {signal }}$ is then defined as:

$$
\sigma=\sqrt{\chi^{2}\left(n_{\text {signal }}(0)\right)}
$$

where $n_{\text {signal }}(0)$ is the signal yield fixed to zero in the fit.

\subsubsection{Probability Distribution Function (PDF) Parameteri- zation}

Table 4.7 shows the parametrization of the PDFs using the following functions:

- $\mathrm{BG}=$ Bifurcated Gaussian

- $\mathrm{G}=$ Gaussian

- $\mathrm{p} 0=$ constant

- $\mathrm{p} 1$ = linear function

- $\mathrm{V}=$ Voigtian, a convolution of a Gaussian and a Breit-Wigner distributions

- $\mathrm{A}=$ ARGUS (described in Ref. [141])

The parameters which are floated in the final fit are indicated under each of the relevant $\mathrm{PDF}$ categories. $m_{\mathrm{ES}}$ and $\Delta E$ means are floated for the signal. The width of the narrower $J / \psi$ Gaussian component is also floated while the other Gaussian widths in the $J / \psi$ and $\eta_{c}$ fit have a fixed ratio to the floated one. Typical distributions for the combinatorial background, signal and self-cross-feed, and $J / \psi, \eta_{c}$ and $\Lambda_{c}^{+}$PDFs are shown in Figures 4.3-4.5. The correlations between the variables used in the ML fit are negligible, thus one-dimensional PDFs can be used to describe event selection variables in all categories. 
Table 4.7: The PDF parametrization of $B \rightarrow p \bar{p} h$ signal and background.

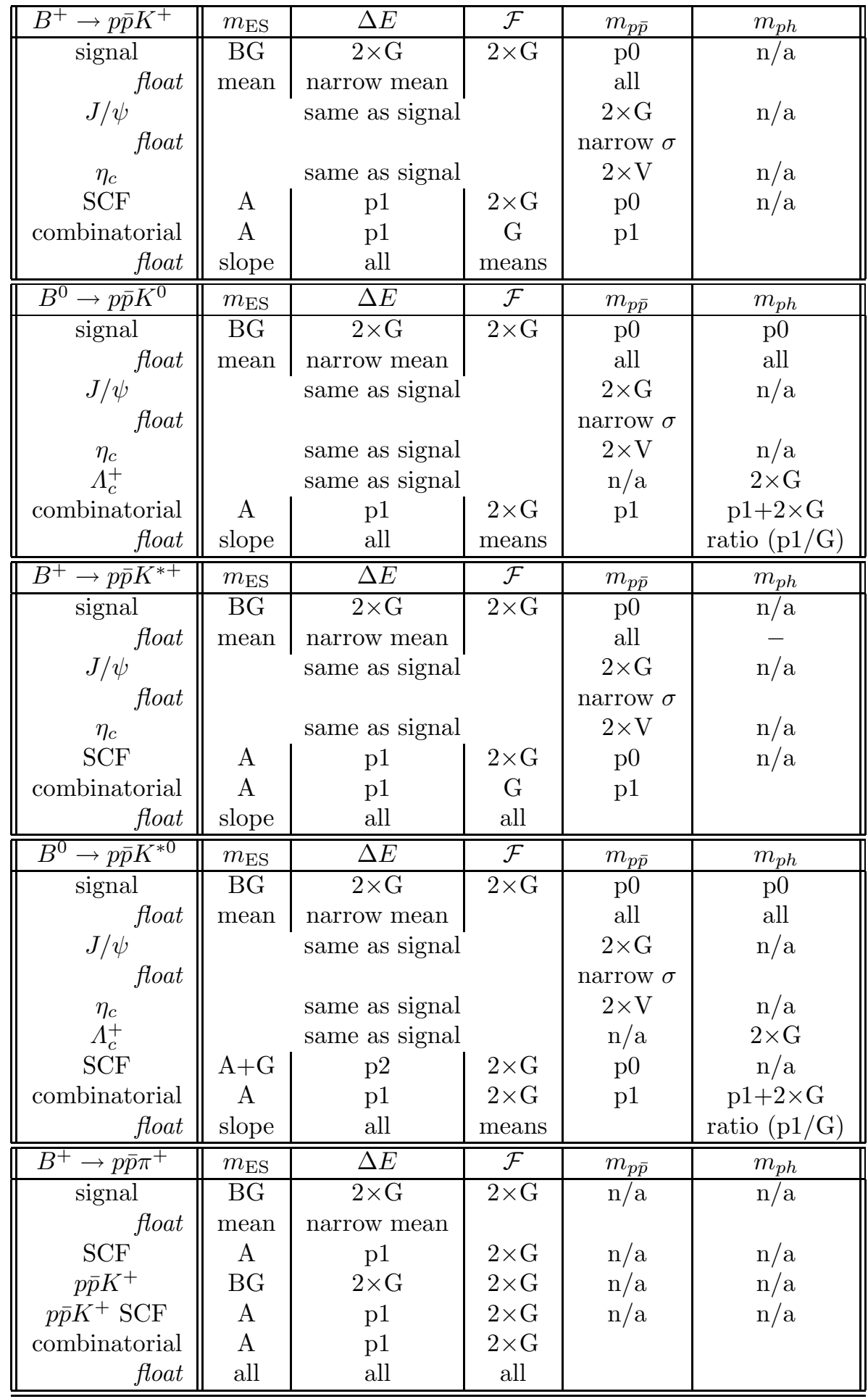



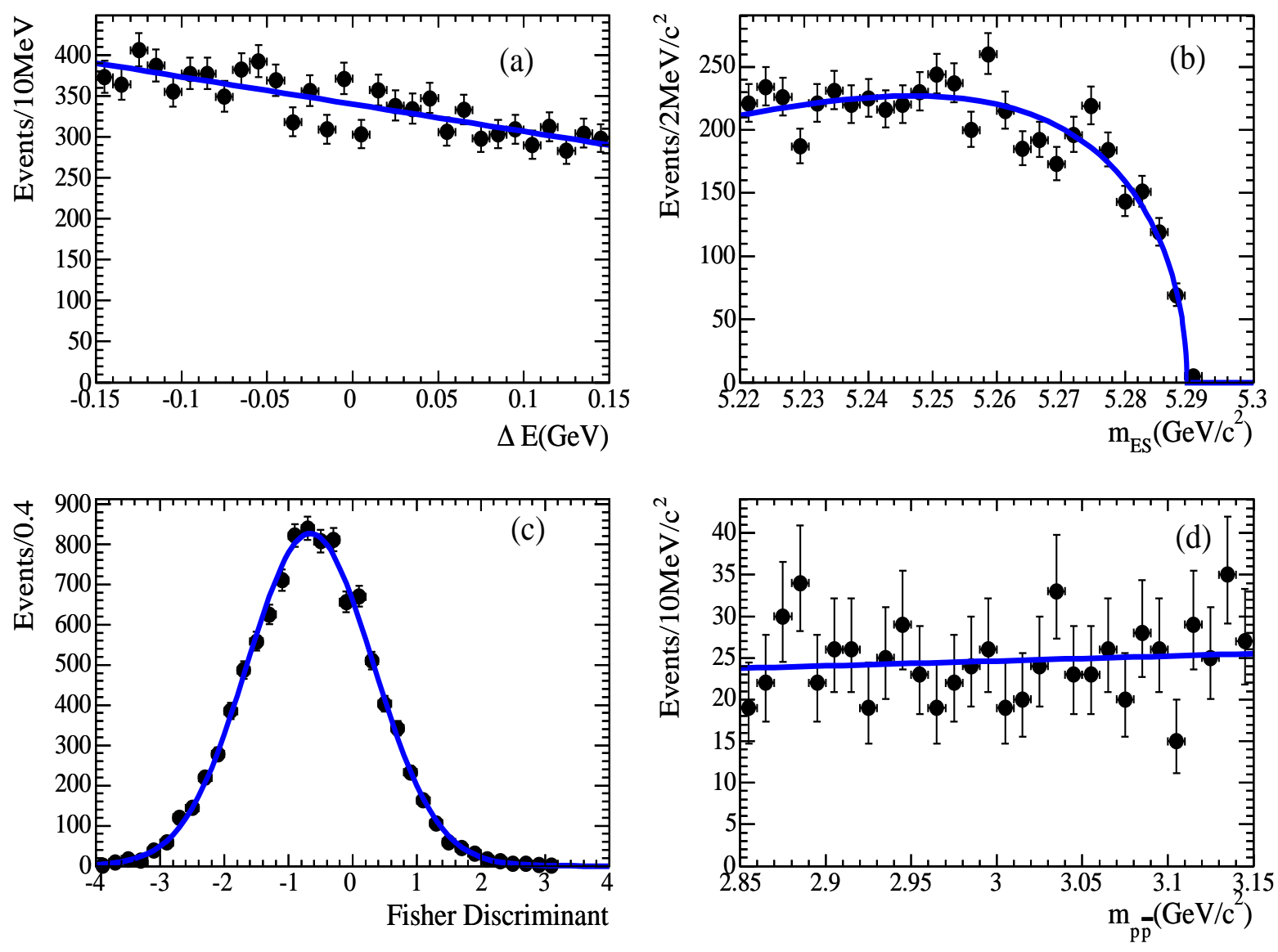

Figure 4.3: PDFs for background in $B^{+} \rightarrow p \bar{p} K^{*+}$ mode: (a) $\Delta E$, (b) $m_{\mathrm{ES}}$, (c) Fisher and (d) $m_{p \bar{p}}$. 

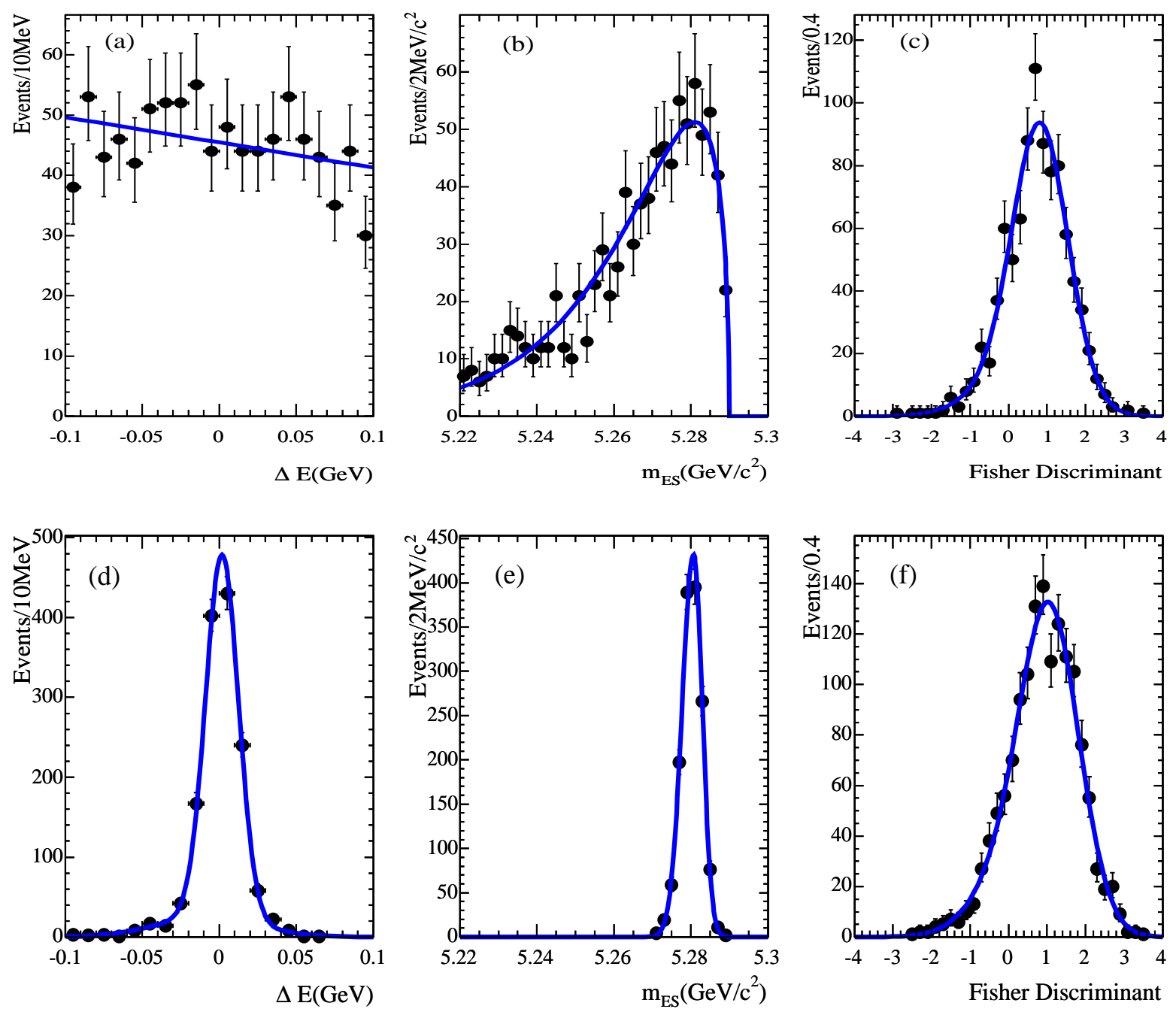

Figure 4.4: PDFs for signal events in $B^{+} \rightarrow p \bar{p} K^{*+}$ mode. Self-cross-feed: (a) $\Delta E$, (b) $m_{\mathrm{ES}}$, (c) Fisher and truth-matched: (d) $\Delta E$, (e) $m_{\mathrm{ES}}$, (f) Fisher. 

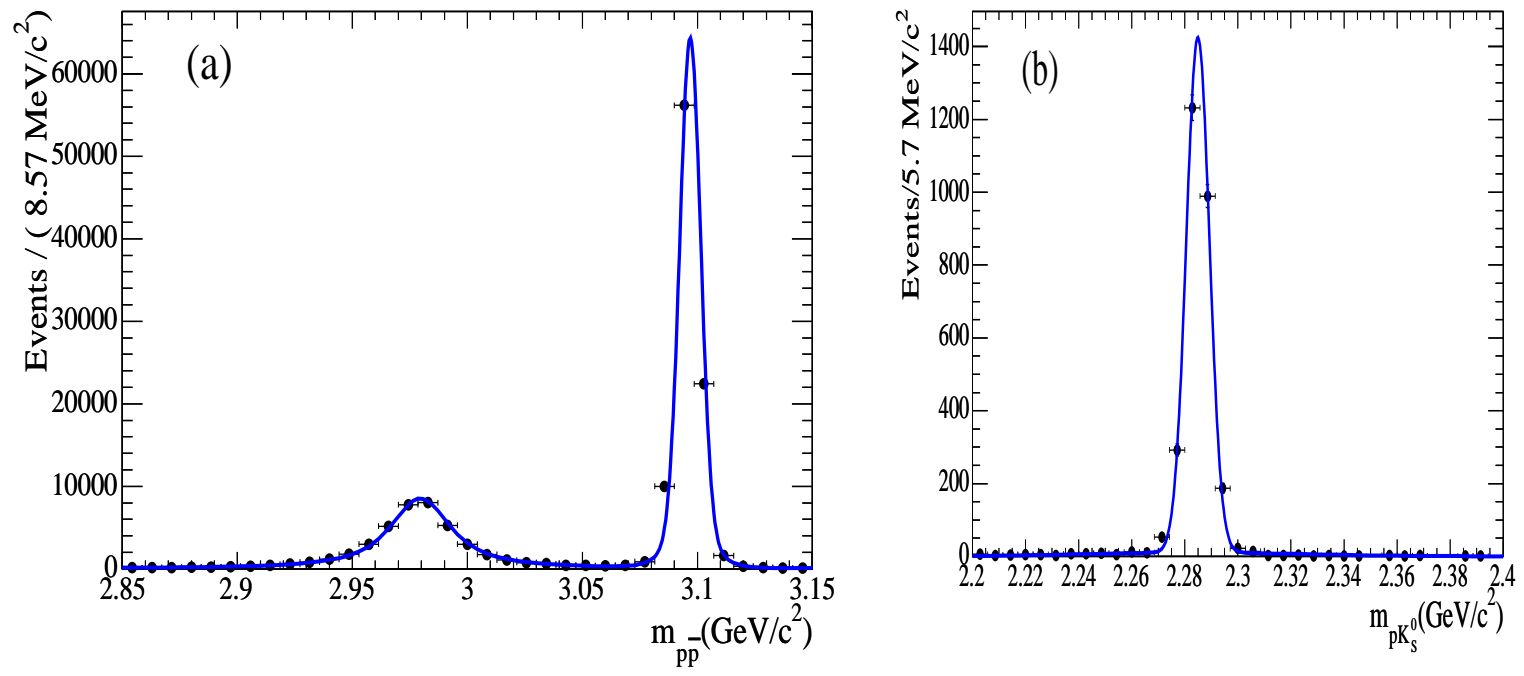

Figure 4.5: PDFs for peaking $B$ background in $B^{0} \rightarrow p \bar{p} K^{0}$ mode: (a) $m_{p \bar{p}}: 2 \times$ Gaussian $(J / \psi)+2 \times$ Voigtian $\left(\eta_{c}\right)$ and (b) $m_{p K_{S}^{0}}: 2 \times$ Gaussian.
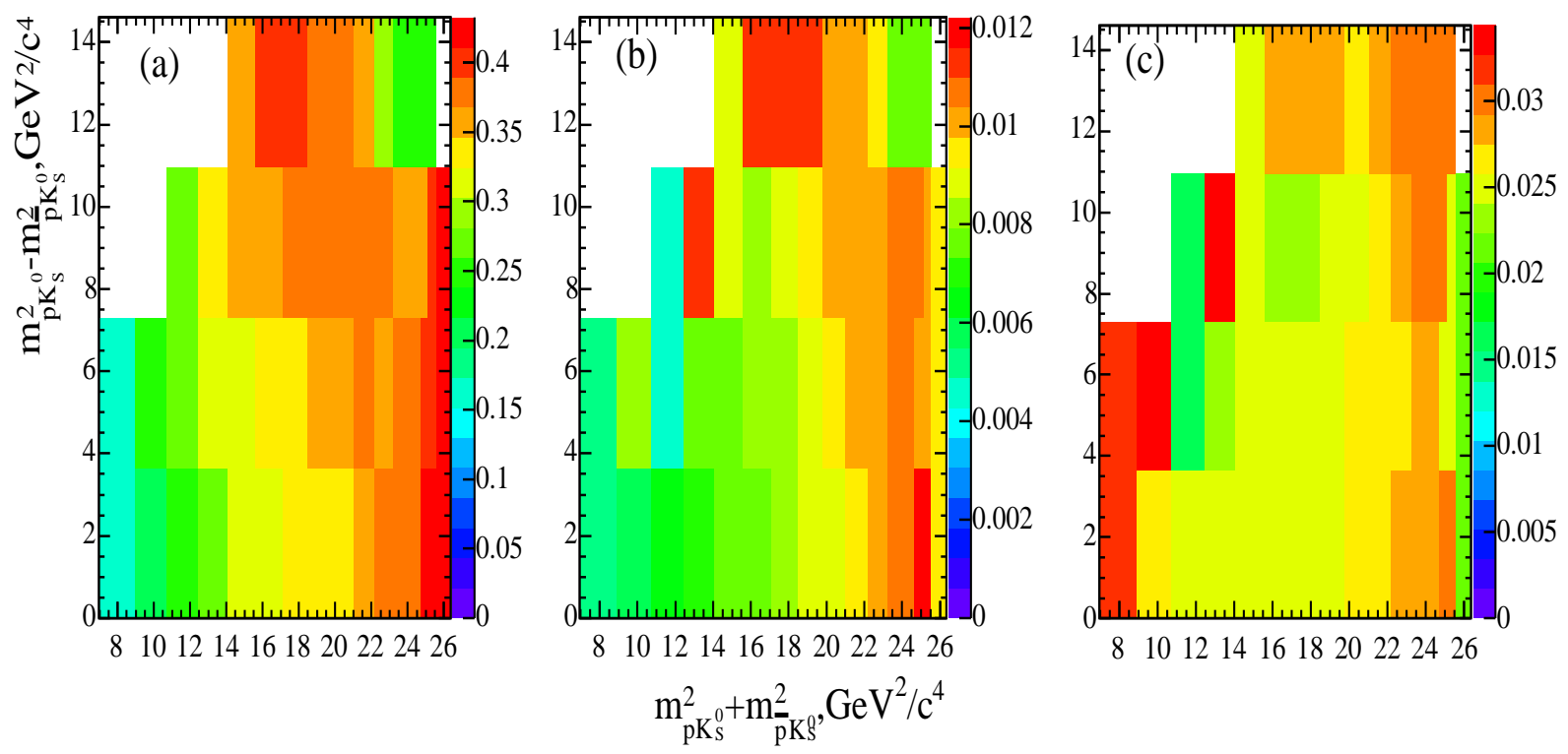

Figure 4.6: (a) Signal efficiency; (b) absolute error on the signal efficiency; (c) relative error on the signal efficiency as a function of the Dalitz plot in $B^{0} \rightarrow p \bar{p} K^{0}$ mode. 


\subsubsection{Signal Efficiency}

For the branching fraction calculation, we must find the efficiency for each event as a function of its Dalitz plot location. To facilitate the Dalitz binning, we construct the Dalitz plot as $\left|m^{2}(p h)-m^{2}(\bar{p} h)\right|$ versus $m^{2}(p h)+m^{2}(\bar{p} h)^{2}$. This transformation has the advantages of collapsing the symmetry of the plot (resulting in higher statistics in each bin), rotating the plot so that the horizontal axis is a function of $m_{p \bar{p}}$, and making one edge of the plot adjacent to zero exactly.

We bin the transformed Dalitz plot into variable bin widths designed to both capture the variation in efficiency across the plot and average out falsely elevated efficiencies at the edges of the plot. To calculate the signal efficiency, the number of events passing the selection criteria in each bin is divided by the number of original Monte-Carlo candidates in that bin. The resulting plot for $B^{0} \rightarrow p \bar{p} K^{0}$ mode is shown in Figure 4.6. The efficiency variation is fairly smooth across the Dalitz plot.

An alternative Dalitz binning scheme is used to quantify the systematic error associated with the Dalitz binning. This version of the Dalitz binning has more bins (approximately twice as many in the vertical direction) than the standard version shown in Figure 4.6. The difference in the branching fraction between the two Dalitz binning schemes is assigned as a systematic error.

\subsection{Systematic Errors}

Using data, Monte Carlo samples, and toy samples generated from the PDFs, we quantify the systematic errors on the measurement. We describe the process in detail for the $B \rightarrow p \bar{p} K_{S}^{0}$ mode; the systematic uncertainties for the other modes are calculated in the same manner.

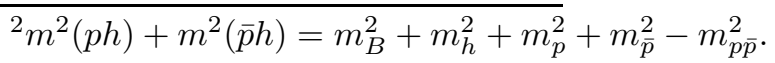




\subsubsection{Fit Validation with Monte Carlo Samples}

\section{PDF Parameter Errors}

For fit validation, we use our PDFs as parent distributions to generate toy data samples which are then fit to the PDFs. Each toy data sample is determined by Poisson smearing around a specified mean $\mu$. The probability for each event to be in a given yield category is given by the the ratio $\mu_{i} / \mu$, where the index $i$ refers to one of the yield categories (signal, self-cross-feed, combinatoric background, etc). To quantify the dependence of the fit signal yield on the PDF parameters, we perform a series of fits to toy data samples, with a set of 100 toy samples for each PDF parameter. For each toy data sample we perform a fit with one of the PDF parameters shifted by its $1 \sigma$ error and a separate fit with the parameter at its normal value. We fit a Gaussian to the distribution of percent change in signal yield for each PDF parameter.

We add the resulting errors together, taking into account the correlations between PDF parameters,

$$
\sigma_{x y}^{2}=\sum_{i} \sum_{j} M_{i j}
$$

to obtain a total error for each

$x=\left\{m_{\mathrm{ES}}, \Delta E, \mathcal{F}, m_{p \bar{p}}, m_{p K_{S}^{0}}\right\}$ and $y=\{$ signal, peaking B background, background $\}$.

$M$ is the error matrix:

$$
M_{i j}=\rho_{i j} \sigma_{i} \sigma_{j}
$$

where $\rho$ is the correlation matrix from the PDF parameter fit and $\sigma_{i}$ is the fractional change in signal yield due to PDF parameter $i$. The procedure described above is repeated using the correlation matrices between $\mathrm{PDF}$ variables and the $\sigma_{x y}$ to obtain a total error for each yield category $y$. These errors are then added in quadrature and averaged over three fit regions, giving a total error of $-3.3 /+2.5 \%$. 


\section{Mixed Toy fits}

To test the validity of the ML fit, we employ fits to datasets consisting of a mixture of toy background and Monte Carlo signal events. The goal is to achieve higher level of statistics available using toy events (i.e. multiple similar fits) without depending wholly on the PDFs to generate the events. Therefore, the events for each fit are assembled from Monte Carlo events wherever possible and toy events when necessary.

The background component of the event sample for these fits is a set of toy events generated from the combinatoric PDF. Signal, $\Lambda_{c}^{+}, J / \psi$ and $\eta_{c}$ Monte Carlo are available in quantities far exceeding the number expected in the data. Appropriate numbers of Monte Carlo events for each of these categories are appended to each toy combinatoric event sample. No Monte Carlo event is used more than once. Signal events were selected according to expected $m_{p \bar{p}}$ distribution. The fit data samples are proportioned identically to those for the toy fits which in turn correspond to the event numbers obtained from the fit to the data sample.

The fit bias for this test is $2.8 \%$ for signal, $0.2 \%$ for $\Lambda_{c}^{+}, 1.4 \%$ for $\eta_{c}$ and $0.1 \%$ for $J / \psi$. These values which are taken as the systematic errors.

\subsubsection{Efficiency Corrections and Systematic Errors}

Reconstruction efficiencies determined by the Monte Carlo studies are then corrected by the amount determined from data and Monte Carlo comparisons.

\section{Tracking efficiency corrections}

The Monte Carlo efficiency for track reconstruction and the track quality requirements described in Section 3.1 are cross-checked using data control samples. The stand-alone tracking capability of the SVT allows tracks to be reconstructed independently of DCH information. The efficiency for the DCH reconstruction and quality requirements can then be calculated in data and Monte Carlo simulation for comparisons. For this purpose, three-prong decay of the $\tau$ and $D^{*+} \rightarrow D^{0} \pi^{+}$decays with four prong $D^{0}$ decays are reconstructed requiring all tracks save one to satisfy the track requirements [152]. The efficiency is then obtained by requiring the final 
(excluded) track to satisfy the track requirements. The results are tabulated into an efficiency correction binned by momentum, polar angle, azimuthal angle, and overall event multiplicity. An average track correction is obtained on a track-by-track basis. The correction suggests that the track reconstruction is somewhat less efficient in the data relative to the Monte Carlo simulation. After applying all the corrections a systematic uncertainty on tracking is assigned to be $0.8 \%$ per track, e.g. $1.6 \%$ for two proton tracks. The average correction value for the $B^{0} \rightarrow p \bar{p} K^{0}$ mode is 0.988 .

\section{$K_{S}^{0}$ tracking corrections}

A separate systematic correction for the $K_{S}^{0}$ reconstruction efficiency, which has less stringent track quality requirements, is obtained from the inclusive $K_{S}^{0}$ decays [153]. The yield of $K_{S}^{0}$ normalized by luminosity is compared between the Monte Carlo simulation of the continuum and inclusive $B \bar{B}$ decays and the on- and off-resonance data. A correction is determined for the reconstruction efficiency in bins of momentum and flight distance. It is applied event-by-event in the signal Monte-Carlo simulation of the reconstructed $K_{S}^{0}$ to determine an average correction. The overall correction factor is 0.981 with an error of $2.8 \%$.

\section{PID corrections}

Each correction has an error to account for the limited size and purity of the control sample used in computing that correction. For example, for the proton identification we use a sample of $\Lambda \rightarrow p \pi^{-}$and for the kaon identification the $D^{*+} \rightarrow D^{0} \pi^{+}, D^{0} \rightarrow$ $K^{+} \pi^{-}$sample. The comparison of the particle identification efficiency in the control samples and relevant Monte Carlo samples leads to PID corrections. To obtain the systematic uncertainty associated with this correction we vary the the values of the corrections within their statistical errors. The resulting average correction is 0.961 . The change in the expected signal efficiency due to this variation gives us $-1.2 /+1.0 \%$ systematic error on the PID correction. 


\subsubsection{Monte Carlo statistics}

Systematic errors arise from binning the signal Monte Carlo Dalitz plot to obtain signal efficiencies. The error due to the fact that bins of finite size will not capture the variation in efficiency over the plot with $100 \%$ accuracy, This error, is calculated as described in Section 4.2.2. To calculate the systematic error on the MC statistics, the branching fraction is recalculated by varying the efficiency within its errors. This results in the systematic error of $2.0 \%$ on the MC statistics.

\subsubsection{Pre-selection cuts}

After all the corrections, we compare our $B^{0} \rightarrow p \bar{p} K_{S}^{0}$ signal simulation to a control sample with similar final state topology $\left(B^{0} \rightarrow J / \psi K_{S}^{0} ; J / \psi \rightarrow e^{+} e^{-} / \mu^{+} \mu^{-}\right)$, in order to quantify the ability of the simulation to model the $B$ vertex probability distribution correctly. We find $\frac{\text { Events }_{M C}}{\text { Events }_{\text {Data }}}=0.976 \pm 0.005$, the difference arising due to different selection efficiencies for Monte Carlo and data. We apply a correction of 0.976 to all the events and assign $0.5 \%$ systematic error.

\subsubsection{Fit Region}

The ranges of $\Lambda_{c}^{+}$and charmonium fit regions are varied around the nominal values to study the variation in the fit yields. The sum in quadrature of the difference between the mean of the obtained values and the nominal value, and the error on that mean is taken as systematic error. We assign a systematic error of $5 \%$ to the $\Lambda_{c}^{+}$measurement and $0.9 \%$ to $B^{0} \rightarrow p \bar{p} K_{S}^{0}$ b.f. measurement (using the total number of 90 events for this estimate).

\subsubsection{Summary of Systematic Uncertainties}

Systematic errors and corrections for the charmless branching fractions for all modes are summarized in Table 4.8, for the charmonium decays in Table 4.9, for the $\Lambda_{c}^{+}$ decays in Table 4.10 and for the exotic searches in Table 4.11. 
Table 4.8: Systematic errors (in percent) and vertexing efficiency corrections for all modes.

\begin{tabular}{lcccc} 
Error Source & $p \bar{p} K^{0}$ & $p \bar{p} K^{*+}$ & $p \bar{p} K^{* 0}$ & $p \bar{p} \pi^{+}$ \\
\hline$B \bar{B}$ counting & 1.1 & 1.1 & 1.1 & 1.1 \\
PID efficiency correction & $-1.2 /+1.0$ & 1.6 & $-3.3 /+3.4$ & 2.1 \\
Track reconstruction efficiency & 1.6 & 2.4 & 3.6 & 2.4 \\
$K_{S}^{0}$ correction & 2.8 & $-2.8 /+3.0$ & $\mathrm{n} / \mathrm{a}$ & $\mathrm{n} / \mathrm{a}$ \\
Monte Carlo Statistics & 2.0 & $-3.4 /+4.4$ & $-2.4 /+2.5$ & $-2.6 /+2.5$ \\
Dalitz plot binning & 2.1 & 3.5 & 1.4 & 2.0 \\
Pre-selection & 0.5 & 0.8 & 0.4 & 0.3 \\
Fit Bias & 5.0 & 3.0 & 5.7 & 1.1 \\
PDF Parameterization & 3.0 & $-3.7 /+3.2$ & $-1.9 /+2.2$ & 6.2 \\
Fit Region & 4.4 & 16.3 & 3.6 & - \\
B Bkg / b.f. errors & 1.0 & 0.8 & 2.9 & 0.8 \\
\hline Total $(\%)$ & 8.7 & $-18.2 /+18.3$ & $-9.6 /+9.5$ & 7.9 \\
\hline Vertexing correction & 0.976 & 0.967 & 0.968 & 0.983 \\
\hline
\end{tabular}

Table 4.9: Systematic errors (in percent) and efficiency corrections (cor) for the charmonium decays.

\begin{tabular}{lcccccc} 
Error Source & $J / \psi K^{0}$ & $\eta_{c} K^{0}$ & $J / \psi K^{*+}$ & $\eta_{c} K^{*+}$ & $J / \psi K^{* 0}$ & $\eta_{c} K^{* 0}$ \\
\hline$B \bar{B}$ counting & 1.1 & 1.1 & 1.1 & 1.1 & 1.1 & 1.1 \\
PID eff cor & 1.3 & 1.2 & 1.4 & 1.6 & 3.3 & 2.9 \\
Track reco & 1.6 & 1.6 & 2.4 & 2.4 & 3.2 & 3.2 \\
$K_{S}^{0}$ cor & 1.0 & 1.1 & 1.3 & 1.2 & - & $\mathrm{n} / \mathrm{a}$ \\
MC Statistics & 0.3 & 0.3 & 1.1 & 1.3 & 0.4 & 0.8 \\
Pre-selection & 0.5 & 0.5 & 0.8 & 0.8 & 0.4 & 0.4 \\
Fit Bias & 1.8 & 3.4 & 1.8 & 3.4 & 1.4 & 1.5 \\
PDF Param & 1.4 & 1.1 & $-0.5 /+0.4$ & $-3.3 /+3.8$ & $-0.6 /+0.8$ & $-2.9 /+3.0$ \\
Fit Region & 0.9 & 5.0 & 0.3 & 1.8 & 1.0 & 3.0 \\
\hline Total & 3.6 & 6.7 & -4.0 & $-6.2 /+6.5$ & 5.1 & 6.4 \\
\hline Vtx cor & 0.976 & 0.976 & 0.967 & 0.967 & 0.968 & 0.968 \\
$K_{S}^{0}$ cor & 0.971 & 0.972 & 0.967 & 0.966 & $\mathrm{n} / \mathrm{a}$ & $\mathrm{n} / \mathrm{a}$ \\
\hline
\end{tabular}


Table 4.10: Systematic errors (in percent) and efficiency corrections for the $\Lambda_{c}^{+}$decays.

\begin{tabular}{lcc} 
Error Source & $\bar{p} \Lambda_{c}^{+}\left(p K^{0}\right)$ & $\bar{p} \Lambda_{c}^{+}\left(p K^{* 0}\right)$ \\
\hline$B \bar{B}$ counting & 1.1 & 1.1 \\
PID efficiency correction & 1.6 & 3.6 \\
Track reconstruction efficiency & 1.6 & 3.2 \\
$K_{S}^{0}$ correction & 2.1 & $\mathrm{n} / \mathrm{a}$ \\
Monte Carlo Statistics & 1.2 & 1.0 \\
Pre-selection & 0.5 & 0.4 \\
Fit Bias & 20.0 & 11.0 \\
PDF Parameterization & $-1.4 /+1.2$ & 0.6 \\
Fit Region & 5.0 & 3.4 \\
\hline Total & 21.0 & 12.6 \\
\hline Vertexing correction & 0.969 & 0.968 \\
$K_{S}^{0}$ correction & 0.976 & $\mathrm{n} / \mathrm{a}$ \\
\hline
\end{tabular}

Table 4.11: Systematic errors (in percent) and efficiency corrections for the exotic searches.

\begin{tabular}{lccccc} 
Error Source & $f_{J} K^{+}$ & $f_{J} K^{0}$ & $f_{J} K^{*+}$ & $f_{J} K^{* 0}$ & $\Theta^{+}$ \\
\hline$B \bar{B}$ counting & 1.1 & 1.1 & 1.1 & 1.1 & 1.1 \\
PID efficiency correction & 2.4 & 1.1 & 2.1 & 3.3 & $-5.8 /+6.6$ \\
Track reconstruction efficiency & 2.4 & 1.6 & 3.2 & 2.4 & 1.6 \\
$K_{S}^{0}$ correction & $\mathrm{n} / \mathrm{a}$ & 1.0 & 1.0 & $\mathrm{n} / \mathrm{a}$ & 1.6 \\
Monte Carlo Statistics & 1.3 & 1.1 & 2.3 & 0.8 & 0.3 \\
Pre-selection & 0.3 & 0.5 & 0.8 & 0.4 & 0.5 \\
Total & 3.8 & 2.7 & 4.3 & 4.8 & $-6.3 /+7.1$ \\
\hline Vertexing correction & 0.983 & 0.976 & 0.967 & 0.968 & 0.976 \\
$K_{S}^{0}$ correction & $\mathrm{n} / \mathrm{a}$ & 0.970 & 0.970 & $\mathrm{n} / \mathrm{a}$ & 0.981 \\
\hline Efficiency, \% & 39.5 & 36.5 & 22.0 & 36.3 & 38.1 \\
\hline
\end{tabular}




\subsection{Summary of Results}

The event yields from the maximum likelihood fits are presented in Table 4.12. For graphical presentation, the variable being plotted is removed from the fit, and the data samples are refitted. The $\mathrm{sPlot}^{3}$ for the resulting distribution for the variable of interest (points) together with the corresponding PDFs used in the fit (solid curve) are shown in Figure 4.7 for signal and Figure 4.8 for background.

\subsection{1 $B \rightarrow p \bar{p} h$ Branching Fraction Measurements}

The branching fractions are determined for each of the modes using the same method employed in $B^{0} \rightarrow p \bar{p} K^{0}$ analysis as described by Equation 4.2. The results of the fits are summarized in Table 4.13. Our fitting method (Section 4.4) removes all $\eta_{c}$ and $J / \psi$ (except in the $B^{+} \rightarrow p \bar{p} \pi^{+}$mode) contributions and most of the $\Lambda_{c}^{+}$contributions.

There still might be some remaining $\Lambda_{c}^{+}$background contribution from the $\Lambda_{c}^{+}$ events in the charmonium region. Knowing the relative efficiency of $\Lambda_{c}^{+}$Monte Carlo events inside and outside the charmonium region allows us to calculate the remaining $\Lambda_{c}^{+}$background contribution from the results of the fit.

The remaining unknown background in the Table 4.1 comes from the $\chi_{c 0}$ events. The current upper limits [20] are summarized in Table 4.1. It is possible to estimate $B^{0} \rightarrow \chi_{c 0} K^{0}$ branching fraction using the corresponding $B^{+} \rightarrow \chi_{c 0} K^{+}$branching fraction measurement by $B A B A R[154]^{4}$. The contribution from the $\chi_{c 0}$ events events is ignored for other modes ${ }^{5}$.

From Table 4.1 we estimate the contribution of the remaining charmonium modes $\left(\chi_{c 1}\right.$ and $\left.\psi(2 S)\right)$. Since only an upper limit exits for the $\chi_{c 2}$ mode, it is not subtracted

\footnotetext{
${ }^{3} \mathrm{~A}$ plot of any variable not present in the fit weighted by "signal" or "background" weight as defined by Equation 4.3 is called sPlot.

${ }^{4}$ Because of the isospin symmetry one would expect ratios of the charged and neutral $B$ mesons decaying into $\chi_{c 0}$ and $\chi_{c 1}$ to be equal. Thus we estimate: $\mathcal{B}\left(B^{0} \rightarrow \chi_{c 0} K^{0}\right) \approx \mathcal{B}\left(B^{+} \rightarrow\right.$ $\left.\chi_{c 0} K^{+}\right) \frac{\mathcal{B}\left(B^{0} \rightarrow \chi_{c 1} K^{0}\right)}{\mathcal{B}\left(B^{+} \rightarrow \chi_{c 1} K^{+}\right)}=(1.34 \pm 0.43 \pm 0.13 \pm 0.14) \times 10^{-4} \times \frac{4.0 \pm 1.2}{6.8 \pm 1.1}=(0.08 \pm 0.04) \times 10^{-3}$. This number needs to be multiplied by the $\mathcal{B}\left(\chi_{c 0} \rightarrow p \bar{p}\right)=(0.22 \pm 0.03) \times 10^{-3}$ and results in the expected contributions to the branching fraction from this mode of $(0.018 \pm 0.009) \times 10^{-6}$. Thus the resulting contribution to the absolute systematic error on the $B$ background is $0.009 \times 10^{-6}$.

${ }^{5}$ Note that there are $1.7 \pm 2.4,1.7 \pm 1.7,-0.4 \pm 0.1$ and $-0.9 \pm 0.7$ events in the $\chi_{c 0}$ region for each of the modes shown in Table 4.13 respectively.
} 
Table 4.12: Summary of the resulting yields from the ML fit for all modes.

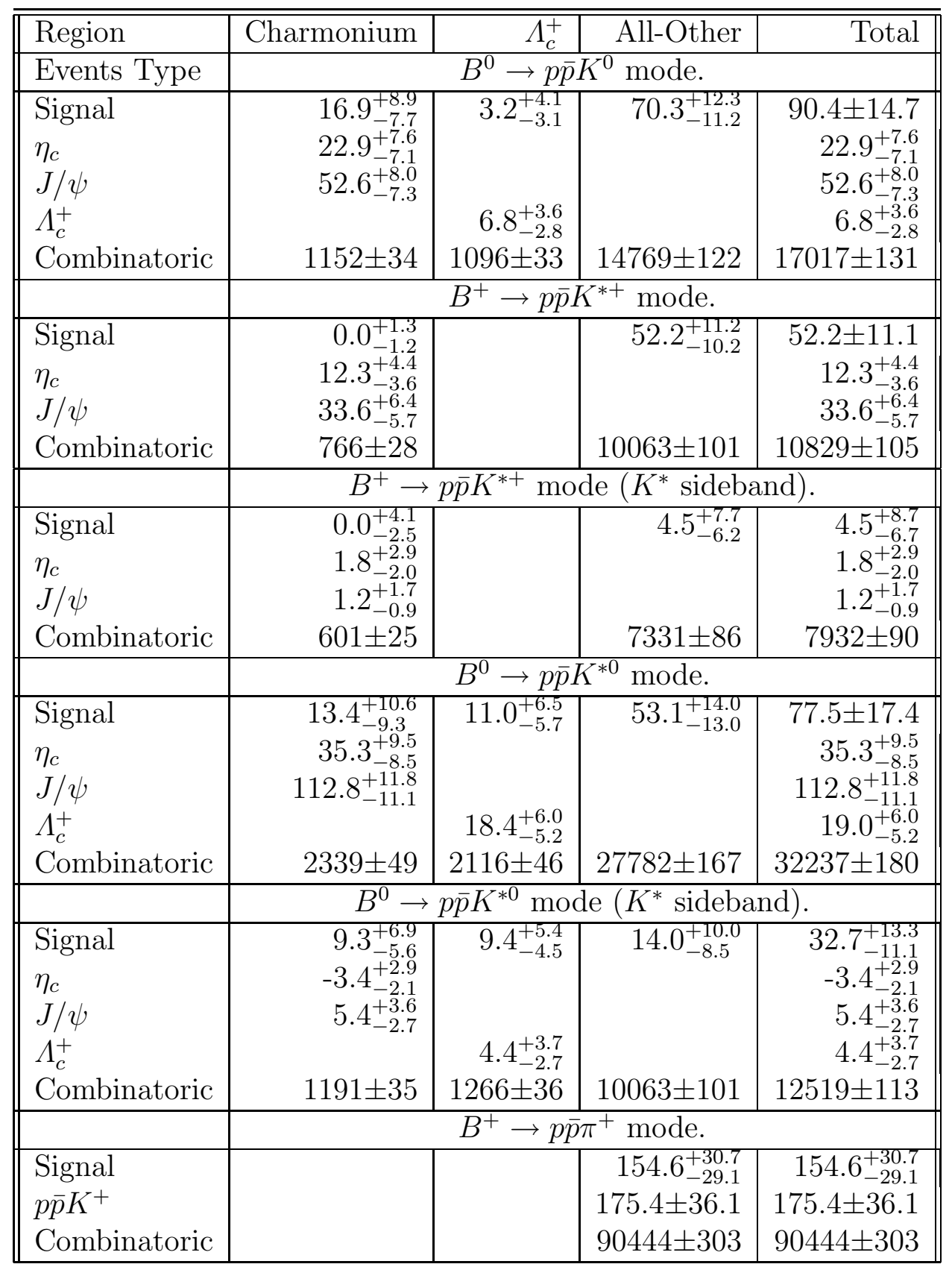



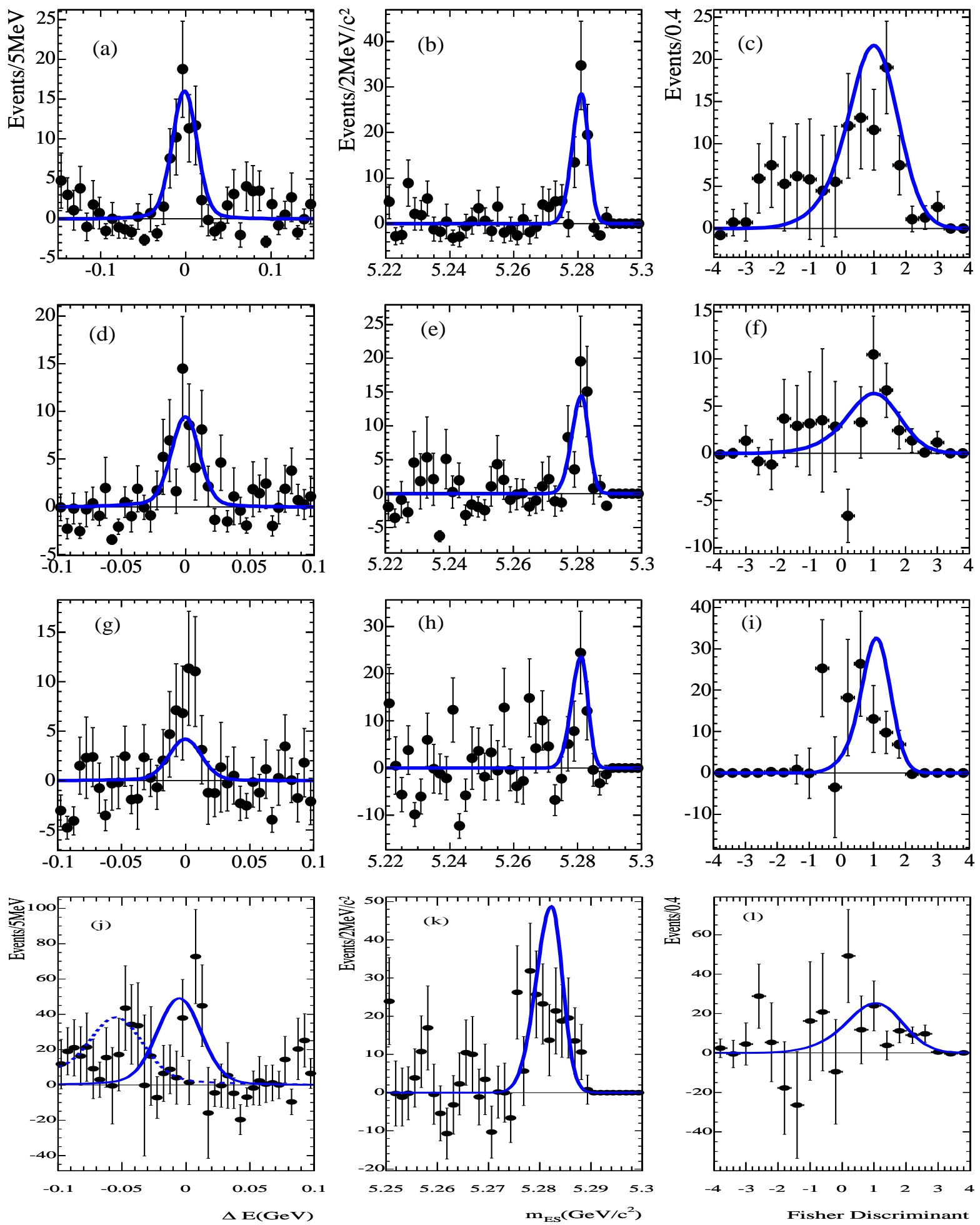

Figure 4.7: Distributions (points with error bars) of $\Delta E, m_{\mathrm{ES}}$, and $\mathcal{F}$ for signal $B^{0} \rightarrow p \bar{p} K^{0}(\mathrm{a})-(\mathrm{c}), B^{+} \rightarrow p \bar{p} K^{*+}(\mathrm{d})-(\mathrm{f}), B^{0} \rightarrow p \bar{p} K^{* 0}(\mathrm{~g})-(\mathrm{i}), B^{+} \rightarrow p \bar{p} \pi^{+}(\mathrm{j})-(\mathrm{l})$ decays using the weighting technique described in Ref. [150]. Solid curves represent the corresponding PDFs used in the fit. 

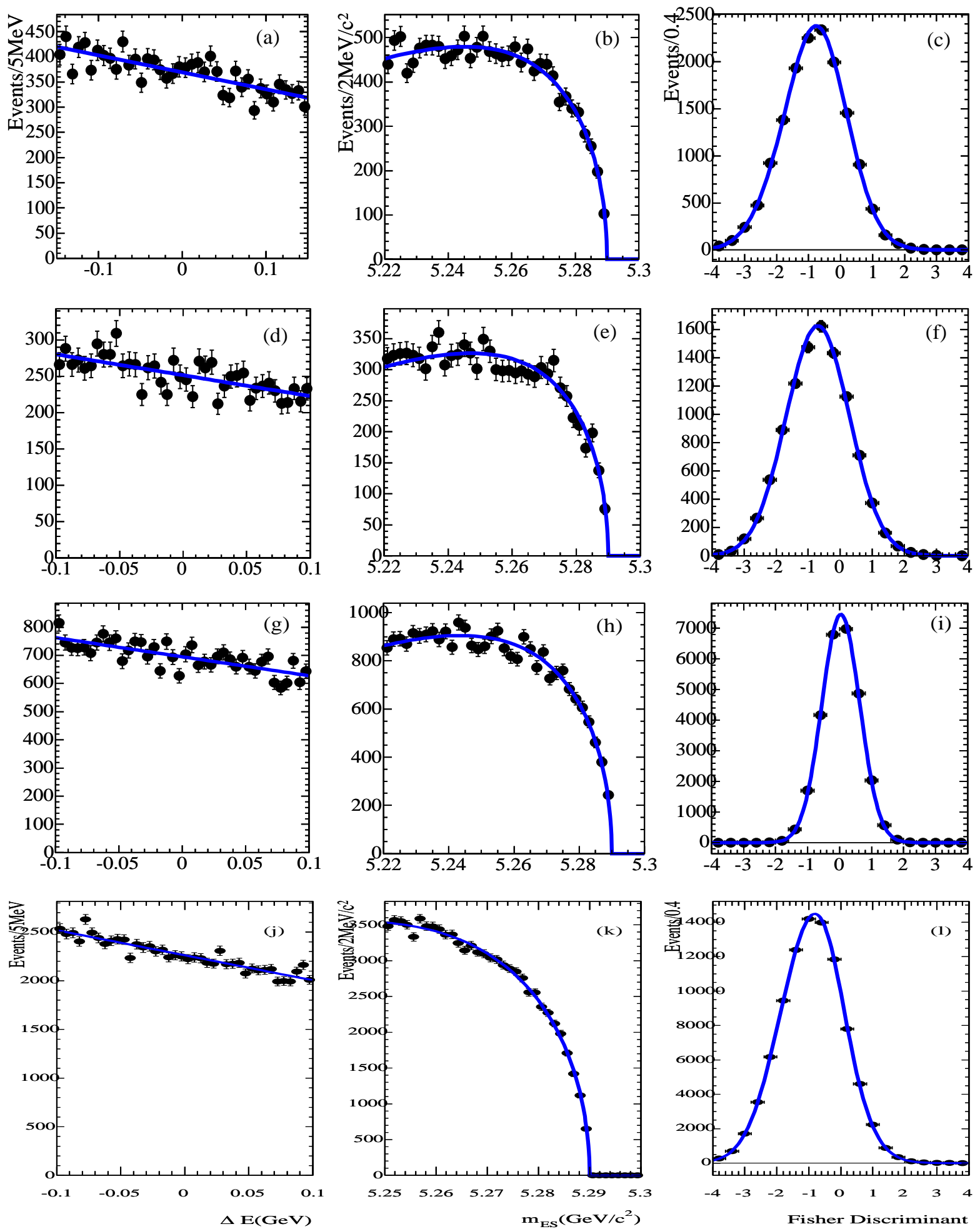

Figure 4.8: Distributions (points with error bars) of $\Delta E, m_{\mathrm{ES}}$, and $\mathcal{F}$ for combinatorial $B^{0} \rightarrow p \bar{p} K^{0}(\mathrm{a})-(\mathrm{c}), B^{+} \rightarrow p \bar{p} K^{*+}(\mathrm{d})-(\mathrm{f}), B^{0} \rightarrow p \bar{p} K^{* 0}(\mathrm{~g})-(\mathrm{i}), B^{+} \rightarrow p \bar{p} \pi^{+}$(j)-(l) decays using the weighting technique described in Ref. [150]. Solid curves represent the corresponding PDFs used in the fit. 
Table 4.13: Non-resonant $B \rightarrow p \bar{p} h$ branching fractions after $B$ background and $K^{*}$ sideband subtractions where applicable.

\begin{tabular}{||l|r|r|r|r||}
\hline \hline & $p \bar{p} K^{0}$ & $p \bar{p} K^{*+}$ & $p \bar{p} K^{* 0}$ & $p \bar{p} \pi^{+}$ \\
\hline Events & $180.8 \pm 29.4$ & $52.2 \pm 14.4$ & $77.5 \pm 17.4$ & $154.6 \pm 28.5$ \\
Efficiency, $\%$ & $35.6 \pm 1.4$ & $18.0 \pm 1.0$ & $23.8 \pm 0.8$ & $45.0 \pm 1.5$ \\
sPlot B.F. $\times 10^{-6}$ & $3.17 \pm 0.53$ & $5.45 \pm 1.49$ & $2.10 \pm 0.47$ & $1.34 \pm 0.32$ \\
\hline Contribution to B.F $\left(\times 10^{6}\right)$ & & & \\
$\Lambda_{c}^{+}$bkg & $0.039 \pm 0.020$ & & $0.091 \pm 0.045$ & \\
$J / \psi$ bkg & & & & $0.102 \pm 0.010$ \\
$\chi_{c 0}$ bkg & $0.018 \pm 0.009$ & & & \\
$\chi_{c 1}$ bkg & $0.03 \pm 0.01$ & $0.02 \pm 0.01$ & $0.024 \pm 0.007$ & \\
$\chi_{c 2}$ bkg & $<0.003$ & $<0.001$ & $<0.002$ & \\
$\psi(2 S)$ bkg & $0.13 \pm 0.02$ & $0.15 \pm 0.04$ & $0.147 \pm 0.026$ & \\
\hline B.F.1 & $2.95 \pm 0.53$ & $5.28 \pm 1.49$ & $1.84 \pm 0.47$ & $1.24 \pm 0.32$ \\
$K^{*}$ SB B.F. & & $0.34 \pm 0.74$ & $0.56 \pm 0.31$ & \\
\hline Final B.F. & $2.95 \pm 0.53 \pm 0.26$ & $4.94 \pm 1.66 \pm 1.00$ & $1.28 \pm 0.56_{-0.17}^{+0.18}$ & $1.24 \pm 0.32 \pm 0.10$ \\
\hline \hline
\end{tabular}

Table 4.14: Comparison of non-resonant $B \rightarrow p \bar{p} h$ branching fractions from Belle $[131,132]$ and this work.

\begin{tabular}{||l|r|r|r|r||}
\hline \hline & $p \bar{p} K^{0}$ & $p \bar{p} K^{*+}$ & $p \bar{p} K^{* 0}$ & $p \bar{p} \pi^{+}$ \\
\hline Belle B.F. & $2.40_{-0.44}^{+0.64} \pm 0.39$ & $10.3_{-2.8}^{+3.6+1.3}$ & $<7.6,90 \%$ CL & $3.06_{-0.62}^{+0.73} \pm 0.37$ \\
\hline This B.F. & $2.95 \pm 0.53 \pm 0.26$ & $4.94 \pm 1.66 \pm 1.00$ & $1.28 \pm 0.56_{-0.17}^{+0.18}$ & $1.24 \pm 0.32 \pm 0.10$ \\
\hline \hline
\end{tabular}

but taken as a contribution to systematic uncertainty.

The line "B.F.1" in Table 4.13 summarizes the values of the non-resonant branching fractions after $B$ background subtraction.

The non- $K^{*}$ background is obtained from the fit identical to that in the one described above performed in the $160<\left|m_{K^{*+}}^{P D G}-m_{K^{*+}}^{r e c o}\right|<240 \mathrm{MeV} / c^{2}$ region. The branching fraction obtained in that region is subtracted from the "B.F.1" resulting in the "Total B.F.", while the errors on the branching fraction in the sideband region are added in quadrature to the other systematic uncertainties.

In Table 4.14 we compare our results with Belle [131],[132]. We observe a new mode $B^{0} \rightarrow p \bar{p} K^{* 0}$ with a significance of $5.1 \sigma$ (for the fit in "all-other" region). 
The branching fractions are approximately two times smaller for $B^{+} \rightarrow p \bar{p} K^{*+}$ and $B^{+} \rightarrow p \bar{p} \pi^{+}$, when compared to the Belle measurements [131], bringing the branching fraction of $B^{+} \rightarrow p \bar{p} K^{*+}$ below $B^{+} \rightarrow p \bar{p} K^{+}$and more in line with theoretical predictions $^{6}$. However, both experiments are in agreement within their errors.

\section{$B \rightarrow p \bar{p} h$ Charge Asymmetry Measurements}

The charge asymmetry is defined as $A_{c h}=\frac{N_{\bar{B}}-N_{B}}{N_{\bar{B}}+N_{B}}$, where $N_{B}$ and $N_{\bar{B}}$ (the event yields in each of the categories of interest) are obtained from the maximum likelihood fit for each $B$ flavor separately. The same fitting procedure is employed as for calculating the branching fraction ${ }^{7}$. The results are summarized in Table 4.15. The measurements for the current modes are consistent with zero within their statistical errors.

\subsubsection{Study of Charmonium Substructure}

\section{$B \rightarrow\left(J / \psi, \eta_{c}\right) h$ Branching Fraction Measurements}

The results of the ML fits for the $\eta_{c}$ and $J / \psi$ region are shown in Figure 4.9. Using the $\eta_{c}$ and $J / \psi$ yields from Table 4.12 and corrections and systematic uncertainties from Table 4.9 we obtain the branching fractions as shown in Table 4.16. The values obtained are consistent with current world averages [20].

We also report evidence for $B^{+} \rightarrow \eta_{c} K^{*+}$ decay. If the number of $\eta_{c}$ events in the fit is fixed at zero, the yields change to:

$$
\begin{aligned}
\mathrm{p} \overline{\mathrm{p}} \mathrm{K}^{*+} \text { Signal Events } & =11.9_{-4.3}^{+5.2} \\
\mathrm{~J} / \psi \mathrm{K}^{*+} \text { Events } & =32.9_{-5.7}^{+6.4} \\
\text { Combinatoric Events } & =767 \pm 28 \\
\eta_{\mathrm{c}} \mathrm{K}^{*+} \text { Events } & =0 \text { (fixed) }
\end{aligned}
$$

The statistical significance of the $\eta_{c} K^{*+}$ signal is calculated from the change in the

\footnotetext{
${ }^{6}$ The $\mathcal{B}\left(B^{+} \rightarrow p \bar{p} K^{+}\right)$was previously reported to be $(6.7 \pm 0.5 \pm 0.4) \times 10^{-6}$.

${ }^{7}$ Note that remaining $B$ background and $K^{*}$ sideband subtractions are not performed in this case.
} 

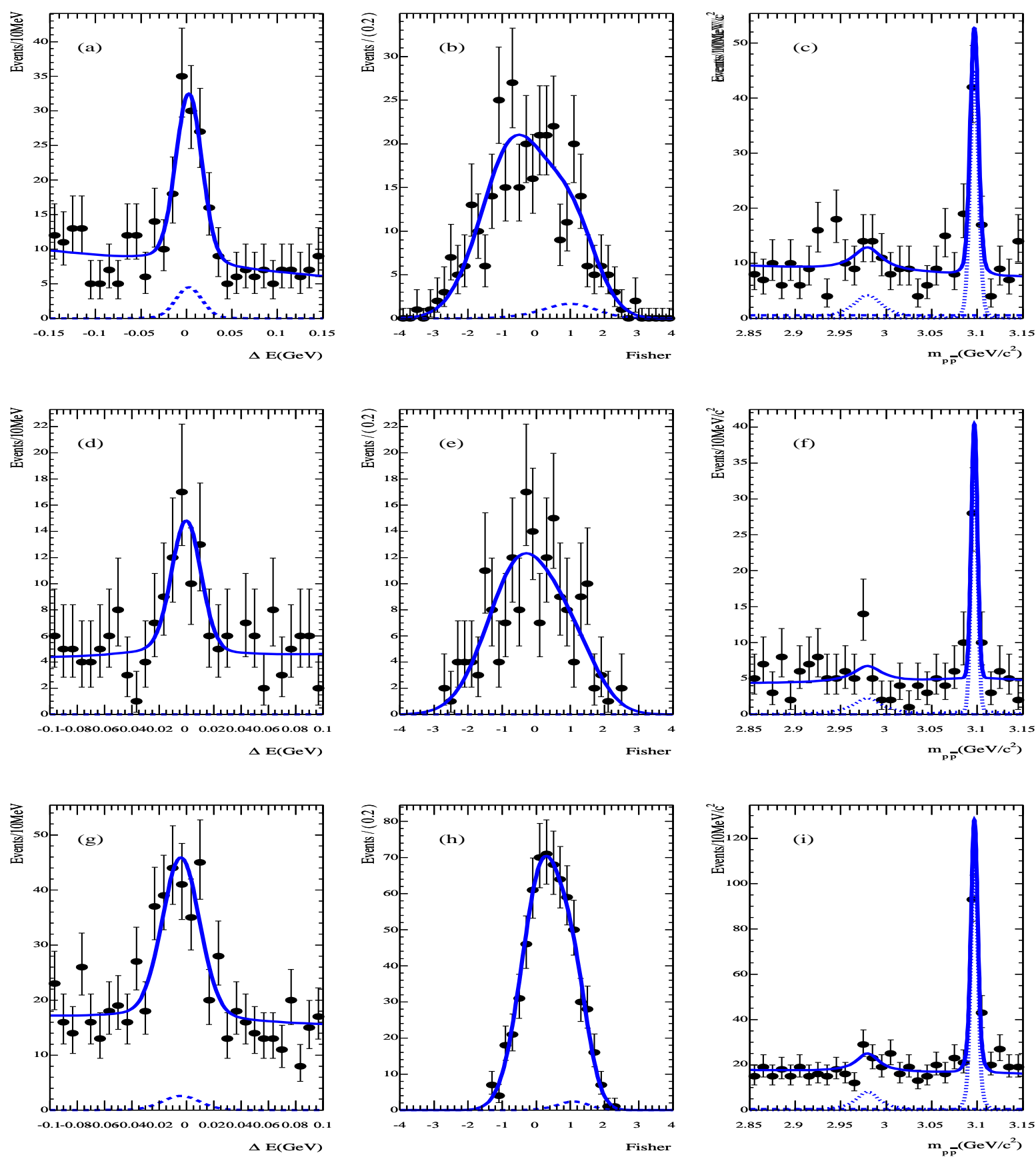

Figure 4.9: The results of the ML fit for $m_{\mathrm{ES}}>5.27 \mathrm{GeV} / c^{2}$ for $B^{0} \rightarrow p \bar{p} K^{0}$ (a)-(c), $B^{+} \rightarrow p \bar{p} K^{*+}(\mathrm{d})$-(f) and $B^{0} \rightarrow p \bar{p} K^{* 0}(\mathrm{~g})$-(i) modes. Solid line represents results of the fit, dashed line is the signal contribution and dotted line shows the $\eta_{c}$ and $J / \psi$ yields in the $m_{p \bar{p}}$ distribution. 
likelihood of the fit from $\ln \left(\mathcal{L}_{\text {max }}\right)=-8058.7$ to $\ln (\mathcal{L}(0))=-8048.0$ resulting in $\sigma=4.6$ (from Equation 4.6).

\subsection{3 $B \rightarrow \Lambda_{c}^{+} \bar{p}$ Branching Fraction Measurement}

$B \rightarrow \Lambda_{c}^{+} \bar{p}$ from $B^{0} \rightarrow p \bar{p} K^{0}$ mode

The result of the ML fit in the $\Lambda_{c}^{+}$region is shown in Figure 4.10(a)-(c). Using the $\Lambda_{c}^{+}$yield fit from Table 4.12 and knowing its efficiency to be $25.4 \pm 0.3 \%^{8}$ and $\mathcal{B}\left(\Lambda_{c}^{+} \rightarrow p K^{0}\right)=(23 \pm 6) \times 10^{-3}$ we obtain $\mathcal{B}\left(B^{0} \rightarrow \Lambda_{c}^{+} \bar{p}\right)=\left(15.1_{-6.2}^{+8.0}(\right.$ stat $) \pm$ $\left.3.2(\text { syst })_{-1.2}^{+1.4}\left(\frac{\mathcal{B}_{\Lambda_{c}^{+} \rightarrow p K^{0}}}{\mathcal{B}_{\Lambda_{c}^{+} \rightarrow p K \pi}}\right)_{-3.2}^{+5.3}\left(\mathcal{B}_{\Lambda_{c}^{+} \rightarrow p K \pi}\right)\right) \times 10^{-6}$.

If the number of $\Lambda_{c}^{+}$events is set to zero, the yields change to:

$$
\begin{aligned}
\mathrm{p} \overline{\mathrm{p}} \mathrm{K}^{0} \text { Signal Events } & =9.2_{-4.1}^{+4.9} \\
\text { Combinatoric Events } & =1096 \pm 33 \\
\overline{\mathrm{p}} \Lambda_{\mathrm{c}}^{+} \text {Events } & =0 \text { (fixed) }
\end{aligned}
$$

The statistical significance of the $\bar{p} \Lambda_{c}^{+}$signal yield is calculated from the change in the likelihood of the fit from $\ln \left(\mathcal{L}_{\text {max }}\right)=-11160.4$ to $\ln (\mathcal{L}(0))=-11154.5$ resulting in $\sigma=3.4$ (from Equation 4.6).

\section{$B \rightarrow \Lambda_{c}^{+} \bar{p}$ from $B^{0} \rightarrow p \bar{p} K^{* 0}$ mode}

The result of the ML fit in the $\Lambda_{c}^{+}$region is shown in Figure 4.10(e)-(f). Using the $\Lambda_{c}^{+}$yield fit from Table 4.12 and knowing its efficiency to be $19.7 \pm 0.2 \%^{9}$ and $\mathcal{B}\left(\Lambda_{c}^{+} \rightarrow p K^{0}\right)=(16 \pm 5) \times 10^{-3}$ we obtain $\mathcal{B}\left(B^{0} \rightarrow \Lambda_{c}^{+} \bar{p}\right)=\left(30.6_{-12.9}^{+15.3}(\right.$ stat $) \pm$ $\left.5.1(\text { syst })_{-3.5}^{+4.5}\left(\frac{\mathcal{B}_{\Lambda_{c}^{+} \rightarrow p K^{* 0}}}{\mathcal{B}_{\Lambda_{c}^{+} \rightarrow p K \pi}}\right)_{-6.3}^{+10.8}\left(\mathcal{B}_{\Lambda_{c}^{+} \rightarrow p K^{* 0}}\right)\right) \times 10^{-6}$.

\footnotetext{
${ }^{8}$ This value includes PID/Tracking corrections only. It needs to be multiplied by 0.976 for the $B$ vertex correction and by 0.969 for the $K_{S}^{0}$ correction.

${ }^{9}$ This value includes PID/Tracking corrections only. It needs to be multiplied by 0.968 for the $B$ vertex correction.
} 
Table 4.15: Summary of the asymmetry study. Resulting yields from the ML fit for all modes.

\begin{tabular}{||l|r|r|r||}
\hline \hline & $N_{\bar{B}}$ & $N_{B}$ & $A_{c h}$ \\
\hline Events Type & \multicolumn{3}{|c|}{$B^{0} \rightarrow p \bar{p} K^{* 0}$ mode. } \\
\hline Signal Charmonium Region & $2.3 \pm 7.2$ & $9.0 \pm 7.5$ & $-0.60 \pm 1.05$ \\
Signal $\Lambda_{c}^{+}$Region & $3.3 \pm 3.4$ & $4.0 \pm 4.3$ & $-0.11 \pm 0.74$ \\
Signal All-Other Region & $29.6 \pm 9.8$ & $28.7 \pm 9.3$ & $0.07 \pm 0.24$ \\
\hline Signal Total & $35.2 \pm 12.6$ & $38.7 \pm 12.7$ & $-0.05 \pm 0.24$ \\
\hline$\eta_{c}$ & $26.3 \pm 7.8$ & $12.1 \pm 5.7$ & $0.37 \pm 0.24$ \\
$J / \psi$ & $65.9 \pm 8.7$ & $46.5 \pm 7.4$ & $0.17 \pm 0.10$ \\
$\Lambda_{c}^{+}$ & $9.9 \pm 3.9$ & $9.0 \pm 3.8$ & $0.05 \pm 0.29$ \\
\hline \hline & \multicolumn{3}{|c||}{$B^{+} \rightarrow p \bar{p} K^{*+}$ mode. } \\
\hline Signal All-Other Region & $34.5 \pm 8.2$ & $20.3 \pm 6.9$ & $0.26 \pm 0.19$ \\
\hline$\eta_{c}$ & $10.7 \pm 3.8$ & $8.2 \pm 2.7$ & $0.13 \pm 0.24$ \\
$J / \psi$ & $19.4 \pm 4.7$ & $15.7 \pm 4.3$ & $0.11 \pm 0.18$ \\
\hline \hline & \multicolumn{3}{|c|}{$B^{+} \rightarrow p \bar{p} \pi^{+}$mode. } \\
\hline Signal & $81.2 \pm 22.2$ & $71.8 \pm 20.3$ & $0.06 \pm 0.020$ \\
\hline \hline
\end{tabular}

Table 4.16: Summary of the resulting branching fractions for $\eta_{c}$ and $J / \psi$ modes (Uncertainties order is as follows: statistical, systematic, due to partial branching fraction correction where appropriate). The following values of branching fractions are used $\mathcal{B}\left(\eta_{c} \rightarrow p \bar{p}\right)=(1.3 \pm 0.4) \times 10^{-3}$ and $\mathcal{B}(J / \psi \rightarrow p \bar{p})=(2.12 \pm 0.10) \times 10^{-3}$.

\begin{tabular}{|c|c|c|c|c|c|}
\hline$p \bar{p} X$ & Eff & \multicolumn{2}{|c|}{$\mathcal{B}\left(B \rightarrow \eta_{c}(p \bar{p}) X\right)\left(10^{-6}\right)$} & \multicolumn{2}{|c|}{$\mathcal{B}\left(B \rightarrow \eta_{c} X\right)\left(10^{-3}\right)$} \\
\hline Mode & $\%$ & Measured & $\mathrm{PDG}$ & Measured & $\mathrm{PDG}$ \\
\hline$p \bar{p} K^{0}$ & 36.3 & $0.83_{-0.26}^{+0.28} \pm 0.05$ & $1.56 \pm 0.71$ & $0.64_{-0.20}^{+0.22} \pm 0.04_{-0.15}^{+0.28}$ & $1.2 \pm 0.4$ \\
\hline$p \bar{p} K^{* 0}$ & 23.7 & $1.09_{-0.25}^{+0.28} \pm 0.06$ & $2.08 \pm 1.11$ & $0.84_{-0.19}^{+0.21} \pm 0.05_{-0.20}^{+0.37}$ & $1.6 \pm 0.7$ \\
\hline$p \bar{p} K^{*+}$ & 15.7 & $1.32_{-0.52}^{+0.67} \pm 0.10$ & - & $1.02_{-0.40-0.07-0.24}^{+0.51+0.08+0.45}$ & - \\
\hline Mode & Eff & \multicolumn{2}{|c|}{$\mathcal{B}(B \rightarrow J / \psi(p \bar{p}) X)\left(10^{-6}\right)$} & \multicolumn{2}{|c|}{$\mathcal{B}(B \rightarrow J / \psi X)\left(10^{-3}\right)$} \\
\hline$p \bar{p} K^{0}$ & $\overline{37.1}$ & $1.87_{-0.26}^{+0.28} \pm 0.07$ & $1.80 \pm 0.08$ & $0.88_{-0.12}^{+0.13} \pm 0.03 \pm 0.04$ & $0.85 \pm 0.04$ \\
\hline$p \bar{p} K^{* 0}$ & 25.0 & $2.87_{-0.30}^{+0.33} \pm 0.15$ & $2.78 \pm 0.20$ & $1.35_{-0.14}^{+0.15} \pm 0.07_{-0.06}^{+0.07}$ & $1.31 \pm 0.07$ \\
\hline$p \bar{p} K^{*+}$ & 17.8 & $3.65_{-0.65}^{+0.74} \pm 0.15$ & $2.86 \pm 0.25$ & $1.72_{-0.31}^{+0.35} \pm 0.07_{-0.08}^{+0.09}$ & $1.35 \pm 0.10$ \\
\hline
\end{tabular}



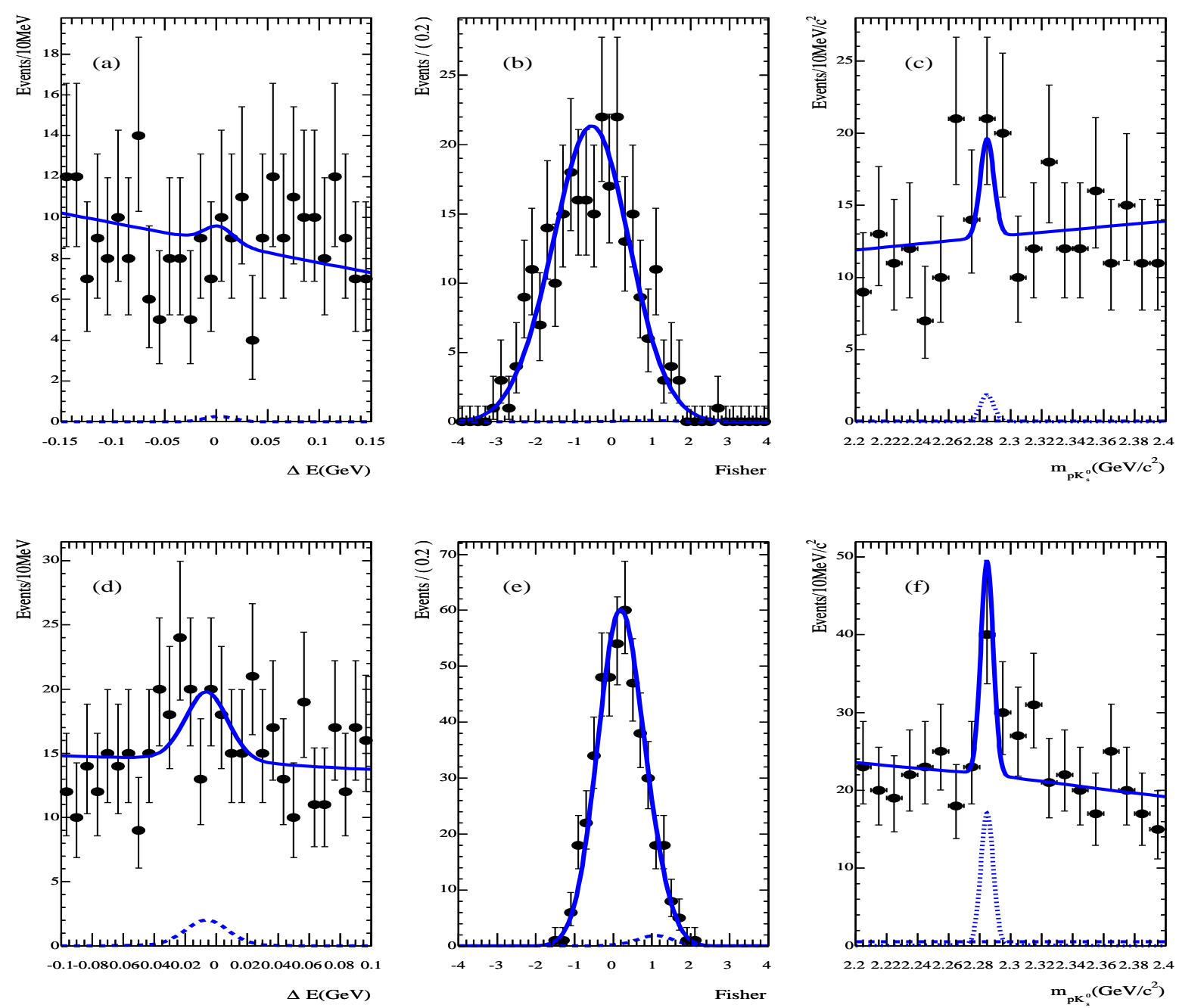

Figure 4.10: The results of the ML fit in the $\Lambda_{c}^{+}$region for $m_{\mathrm{ES}}>5.27 \mathrm{GeV} / c^{2}$ for $B^{0} \rightarrow p \bar{p} K^{0}$ (a)-(c) and $B^{0} \rightarrow p \bar{p} K^{* 0}$ (d)-(f) modes. Solid line represents results of the fit, dashed line is the signal contribution and dotted line shows the $\bar{p} \Lambda_{c}^{+}$yield in the $m_{p K_{S}^{0}}$ distribution. 
If the number of $\Lambda_{c}^{+}$events is set to zero, the yields change to:

$$
\begin{aligned}
\mathrm{p} \overline{\mathrm{p}} \mathrm{K}^{* 0} \text { Signal Events } & =22.8_{-6.5}^{+7.6} \\
\text { Combinatoric Events } & =2122 \pm 46 \\
\overline{\mathrm{p}} \Lambda_{\mathrm{c}}^{+} \text {Events } & =0(\text { fixed })
\end{aligned}
$$

The statistical significance of the $\bar{p} \Lambda_{c}^{+}$signal yield is calculated from the change in the likelihood of the fit from $\ln \left(\mathcal{L}_{\text {max }}\right)=-25132.9$ to $\ln (\mathcal{L}(0))=-25119.9$ resulting in $\sigma=5.1$ (from Equation 4.6).

\section{$B \rightarrow \Lambda_{c}^{+} \bar{p}$ branching fraction measurement combining the modes}

Averaging results for all the modes and adding the errors in quadrature (except the systematic error on $B$-counting), we obtain branching fraction for $\mathcal{B}\left(B^{0} \rightarrow \Lambda_{c}^{+} \bar{p}\right)=$ $\left(22.9_{-7.2}^{+8.6}(\right.$ stat $\left.) \pm 3.0(\text { syst })_{-1.9}^{+2.4}\left(\Lambda_{c}^{+} \text {b.f }\right)_{-4.7}^{+8.0}\left(\mathcal{B}_{\Lambda_{c}^{+} \rightarrow p K \pi}\right)\right) \times 10^{-6}$. This measurement is consistent with the current value of $\mathcal{B}\left(B^{0} \rightarrow \Lambda_{c}^{+} \bar{p}\right)=\left(21.9_{-4.9}^{+5.6} \pm 3.2 \pm 5.7\right) \times 10^{-6}$ based on a single measurement by Belle [125].

\subsection{4 $\mathcal{B}\left(B^{0} \rightarrow \Theta(1540)^{+} \bar{p}\right)$ Upper Limit Calculation}

As was suggested in Ref. [117] we search for a pentaquark baryon candidate $\Theta^{+}$, in the $m_{p K_{S}^{0}}$ mass distribution of $B^{0} \rightarrow p \bar{p} K^{0}$ decays. A search for $\Theta^{+}$has been performed by Belle in $140 \mathrm{fb}^{-1}[132]$, and they set an upper limit on $\mathcal{B}\left(B^{0} \rightarrow \Theta(1540)^{+} \bar{p}\right) \times \mathcal{B}(\Theta \rightarrow$ $p K_{S}^{0}$ ) (in the region $1.53<m_{p K_{S}^{0}}<1.55 \mathrm{GeV} / c^{2}$ ) of $0.23 \times 10^{-6}$ at $90 \% \mathrm{CL}$. If $\Theta^{+}$ decays strongly, there are only two possible decays modes: $n K^{+}$and $p K^{0}$. For this measurement we assume $\mathcal{B}\left(\Theta \rightarrow p K_{S}^{0}\right)=25 \%$.

From dedicated signal Monte-Carlo we determine that the $\Theta^{+}$invariant mass resolution is represented by a sum of two Gaussian functions with common center. The resolutions of the core (tail) Gaussian are $0.95(2.32) \mathrm{MeV} / \mathrm{c}^{2}$ and the wider Gaussian contributes $19 \%$ of the total. The overall resolution, defined as the FWHM of the resolution function divided by 2.355 is $1 \mathrm{MeV} / c^{2}$ at the $\Theta^{+}$mass of $1.54 \mathrm{GeV} / c^{2}$. The $\Theta^{+}$pentaquark signal efficiency is $30.8 \pm 0.1 \%$. 


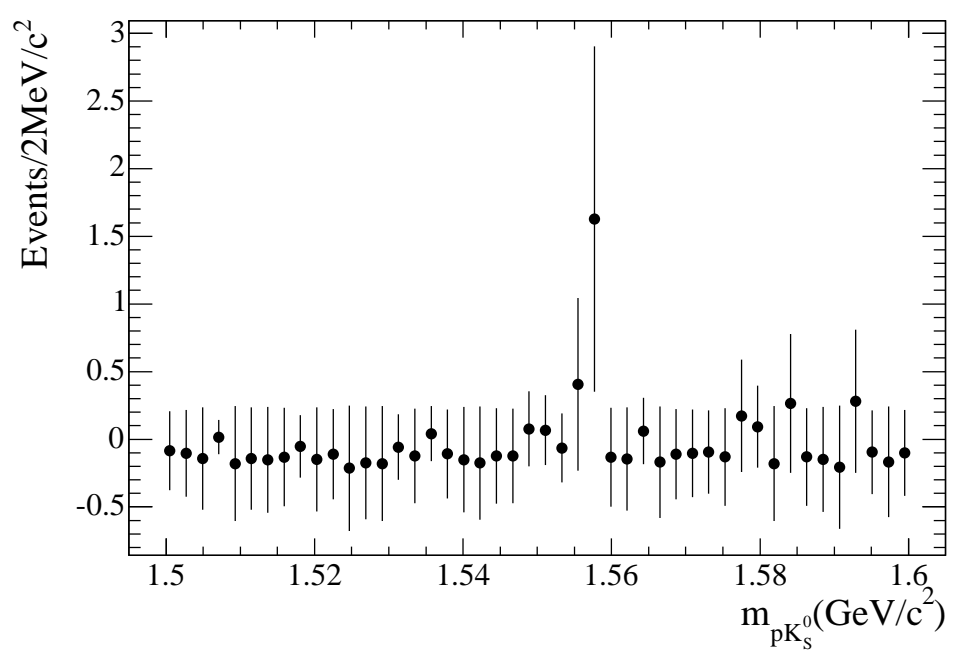

Figure 4.11: sPlot of the $p K_{S}^{0}$ mass projections in $\Theta^{+}$mass region for $B^{0} \rightarrow$ $p \bar{p} K^{0}$ signal events.

The sPlot of the $p K_{S}^{0}$ mass projections in $\Theta^{+}$mass region is shown in Figure 4.11. The plot is fairly smooth (and has no sign of $\Theta^{+}$) with a small enhancement of $\approx 2$ events at $1.557 \mathrm{GeV} / c^{2}$. A Bayesian approach is used to calculate the U.L. at $90 \%$ C.L. as a function of $m_{p K^{+}}$, assuming Poisson-distributed events in the absence of background. As there are no events anywhere but at $1.557 \mathrm{GeV} / c^{2}$ we obtain the following upper limit on the $\mathcal{B}\left(B^{0} \rightarrow \Theta(1540)^{+} \bar{p}\right)$ at $90 \%$ c.l. ${ }^{10}$ :

$$
\frac{2.3}{232 \times 10^{6} \cdot 0.308 \cdot 0.25 \cdot 0.6895 \cdot 0.976 \cdot 0.981} \times(1.071)=0.20 \times 10^{-6} .
$$

The corresponding value for the mass region around $1.557 \mathrm{GeV} / c^{2}$ is $0.47 \times 10^{-6}$. These values are consistent with and improve on the Belle's upper limit of $\mathcal{B}\left(B^{0} \rightarrow\right.$ $\left.\Theta(1540)^{+} \bar{p}\right)<0.92 \times 10^{-6}[132]$.

\footnotetext{
${ }^{10}$ Systematic errors of $7.1 \%$ is included. 0.976 is $B$ vertex cut correction. 0.981 is $K_{S}^{0}$ efficiency correction.
} 

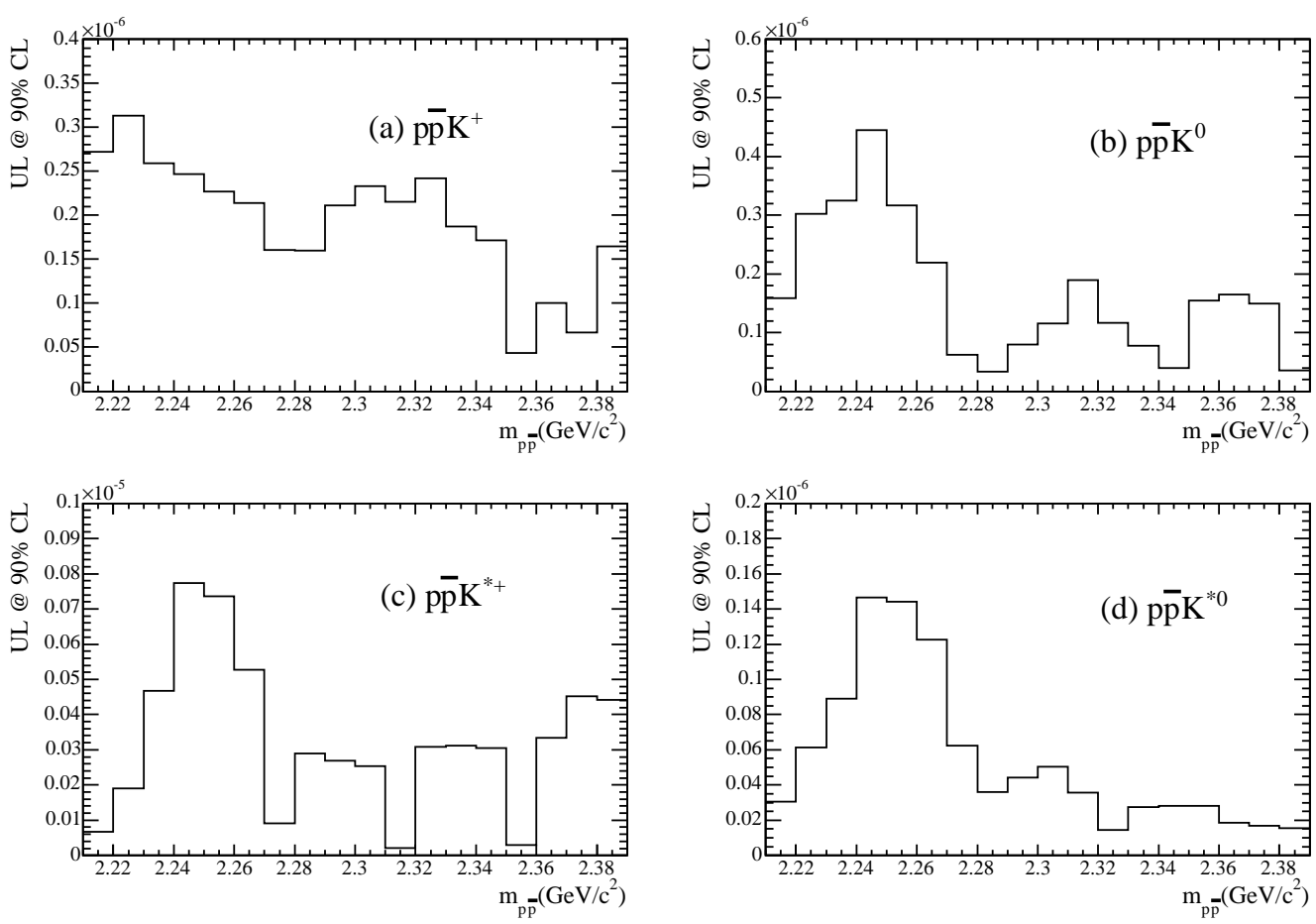

Figure 4.12: Upper limits at 90\% CL on the product of branching fractions $\mathcal{B}(B \rightarrow$ $\left.f_{J}(2220) X\right) \times \mathcal{B}\left(f_{J}(2220) \rightarrow p \bar{p}\right)$ for $(\mathrm{a}) B^{+} \rightarrow p \bar{p} K^{+},(\mathrm{b}) B^{0} \rightarrow p \bar{p} K^{0}$, (c) $B^{+} \rightarrow$ $p \bar{p} K^{*+}$ and $(\mathrm{d}) B^{0} \rightarrow p \bar{p} K^{* 0}$ modes.

\subsubsection{Search for glueballs in $B \rightarrow p \bar{p} h$ decays}

One theoretical conjecture [45] suggests a possible presence of $f_{J}(2220)$ resonance in the baryonic $B$ decays. We perform scan through $2.2<m_{p \bar{p}}<2.4 \mathrm{GeV} / c^{2}$ region with a $30 \mathrm{MeV} / c^{2}$ mass window in the final states with $b \rightarrow s$ transition. The results of the scan are shown in Figure 4.12. The largest upper limits at 90\% CL on the product of branching fractions ${ }^{11}$ are found to be $\mathcal{B}\left(B \rightarrow f_{J}(2220) X\right) \times \mathcal{B}\left(f_{J}(2220) \rightarrow p \bar{p}\right)<$ $3.1(4.5,7.7$ and 1.5$) \times 10^{-7}$ for $X=K^{+}, K^{0}, K^{*+}$ and $K^{* 0}$, respectively.

The corresponding upper limit on the product of branching fractions from Belle is $\mathcal{B}\left(B^{+} \rightarrow f_{J}(2220) K^{+}\right) \times \mathcal{B}\left(f_{J}(2220) \rightarrow p \bar{p}\right)<4.1 \times 10^{-7}$ with $20 \mathrm{MeV} / c^{2}$ mass window. The theoretical expectations are $\sim 1 \times 10^{-6}[132]$.

\footnotetext{
${ }^{11}$ We use the following formula to calculate the upper limit: $U L=$ Yield $+1.64 \cdot \Delta Y$ ield $\cdot(1+$ syst $)$.
} 


\subsection{Summary}

With $210 \mathrm{fb}^{-1}$ of data, we measure the branching fractions of $B \rightarrow p \bar{p} h$ final states where $h=K_{S}^{0}, K^{*+}, K^{* 0}, \pi^{+}$. We report the first observation of the $B^{0} \rightarrow p \bar{p} K^{* 0}$ decay with statistical significance of $5.1 \sigma$ and branching fraction of $\left(1.28 \pm 0.56_{-0.17}^{+0.18}\right) \times$ $10^{-6}$. Our branching fraction measurements in the other modes are consistent with those of reported by Belle [131],[132] using $78 \mathrm{fb}^{-1}$ and $140 \mathrm{fb}^{-1}$, respectively. The resulting branching fraction in the $B^{0} \rightarrow p \bar{p} K^{0}$ mode, $(2.95 \pm 0.53 \pm 0.26) \times 10^{-6}$, is higher than measured by previously by Belle. This is expected as this value takes into account the charmonium and $\Lambda_{c}^{+}$regions excluded in Ref. [132]. The values are two times smaller than in Ref. [131] for the $B^{+} \rightarrow p \bar{p} K^{*+}$ and $B^{+} \rightarrow p \bar{p} \pi^{+}$modes at $(4.94 \pm 1.66 \pm 1.00) \times 10^{-6}$ and $(1.24 \pm 0.32 \pm 0.10) \times 10^{-6}$, respectively. We also report the first evidence of the $B^{+} \rightarrow \eta_{c} K^{*+}$ decay at $4.6 \sigma$ statistical significance and confirm Belle's observation [125] of $B \rightarrow \Lambda_{c}^{+} \bar{p}$ in $\Lambda_{c}^{+} \rightarrow p K^{0(*)}$ modes. No evidence is found for the pentaquark candidate $\Theta^{+}$, and we improve the branching fraction upper limits of $0.2 \times 10^{-6}$ by more than a factor of four [132]. We also find no evidence to a presence of $f_{J}(2220)$ glueball candidate in $p \bar{p}$ spectrum and set upper limits on the order of $10^{-7}-10^{-6}$ in modes with $K^{+}, K_{S}^{0}, K^{*+}, K^{* 0}$. 


\section{Chapter 5}

\section{Summary and Outlook}

\subsection{Summary of Branching Fraction Values}

The measurements of branching fractions for the $B \rightarrow p \bar{p} h$ modes from Ref. [131],[132] and this work are summarized in Table 5.1 and compared to those of the two-body mesonic modes from Ref. [149]. In the following discussion only the experimental measurements of $B \rightarrow p \bar{p} h$ presented in this work are used.

One prominent observation is that the ratio of $B^{+} \rightarrow p \bar{p} K^{+}$to $B^{0} \rightarrow p \bar{p} K^{0}$ branching fractions is not unity as seen in two-body meson modes, but it is closer to 2 . This could be explained with an external W-emission diagram (see Figure 1.4) which is not possible for the $K^{0}$ mode. Although this contribution is expected to be small due to double CKM suppression, it may be at the same level as the penguin contributions.

Table 5.1: Summary of the experimental values for the branching fractions $\left(\times 10^{-6}\right)$ of $B \rightarrow p \bar{p} h$ and their comparison to two-body mesonic modes from Ref. [149].

\begin{tabular}{||c||c|c||c|c||}
\hline$h$ & Belle $p \bar{p} h$ & BABAR $p \bar{p} h$ & $\pi^{0} h$ & $\rho^{0} h$ \\
\hline$K^{+}$ & $5.30_{-0.39}^{+0.45} \pm 0.58$ & $6.7 \pm 0.5 \pm 0.4$ & $12.1 \pm 0.8$ & $4.2 \pm 0.6$ \\
$K^{0}$ & $2.40_{-0.44}^{+0.64} \pm 0.39$ & $2.95 \pm 0.53 \pm 0.26$ & $11.5 \pm 1.0$ & $5.1 \pm 1.6$ \\
$K^{*+}$ & $10.3_{-2.8}^{+3.6+1.3}$ & $4.94 \pm 1.66 \pm 1.00$ & $6.9 \pm 2.3$ & $10.6_{-3.5}^{+3.8}$ \\
$K^{* 0}$ & $<7.6,90 \% \mathrm{CL}$ & $1.28 \pm 0.56_{-0.17}^{+0.18}$ & $1.7 \pm 0.8$ & $<2.6$ \\
$\pi^{+}$ & $3.06_{-0.62}^{+0.73} \pm 0.37$ & $1.24 \pm 0.32 \pm 0.10$ & $5.5 \pm 0.6$ & $8.7_{-1.1}^{+1.0}$ \\
\hline
\end{tabular}


There may also be a contribution due to interference between different diagrams. The pole model [122] predicts $B^{0} \rightarrow p \bar{p} K^{0}$ rate an order of magnitude smaller that that of $B^{+} \rightarrow p \bar{p} K^{+}$. The form-factor model [28] predicts the current-induced parts to be identical in both modes while the transition part of $B^{0} \rightarrow p \bar{p} K^{0}$ can be $\ll 1$, $\approx 1$, or $\gg 1$ (compared to $B^{+} \rightarrow p \bar{p} K^{+}$), depending on which contribution $\left(C_{P}, C_{A}\right.$ or $\left.C_{V 5}\right)$ is dominant (see discussion in Section 1.6). As the $B^{+} \rightarrow p \bar{p} K^{+}$branching fraction is twice as large as that of $B^{0} \rightarrow p \bar{p} K^{0}$ one could conclude that the $C_{V 5}$ contribution is not dominant. The $B^{+} \rightarrow p \bar{p} K^{*+}$ branching fraction is also larger (by a factor of four) that that of $B^{0} \rightarrow p \bar{p} K^{* 0}$, similar to the pattern observed in the two-body mesonic modes.

The $K^{*}$ modes are consistently smaller than the $K$ modes in both the charged and neutral cases. This seems to be the case for the $\pi^{0} h$ modes as well, but not for the $\rho^{0} h$ modes.

The $B^{+} \rightarrow p \bar{p} \pi^{+}$branching fraction is lower than that of the $B^{+} \rightarrow p \bar{p} K^{+}$as expected because the $b \rightarrow u$ transition at tree level is suppressed compared to the $b \rightarrow s$ penguin. This is similar to what is observed in the $\pi^{0} h$ modes but contrary to what is observed in $\rho^{0} h$.

Overall, the theoretical calculations of the baryonic $B$ decays are not very precise and the current measurements of the branching fractions of all four $p \bar{p} K$ modes should help to improve our understanding of those decays.

\section{$5.2 \quad$ Study of the $B \rightarrow p \bar{p} h$ decay dynamics}

For decay dynamics studies, the ML fit is performed using three variables $\left(m_{\mathrm{ES}}, \Delta E\right.$ and Fisher discriminant) simultaneously over the whole Dalitz plot. The resulting Dalitz sPlots for all the modes are shown in Figure 5.1. The main features of the Dalitz plots are expected to be the charmonium resonances (with $J / \psi$ and $\eta_{c}$ bands most prominent), potential $\Lambda_{c}^{+}$bands in $B^{0} \rightarrow p \bar{p} K^{0}$ and $B^{0} \rightarrow p \bar{p} K^{* 0}$ modes, as well as the low $p \bar{p}$ mass enhancements. 

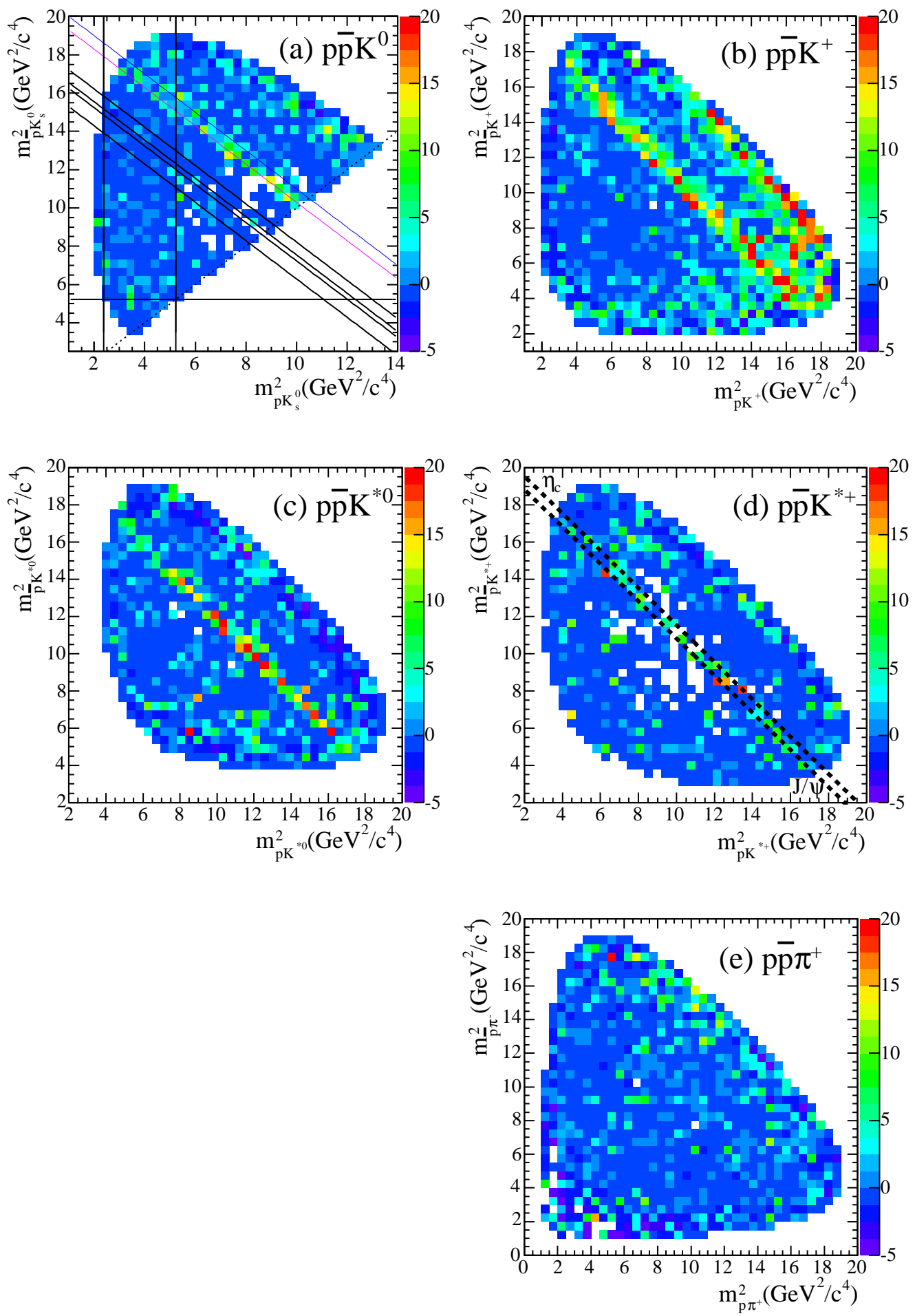

Figure 5.1: Dalitz sPlots for (a) $B^{0} \rightarrow p \bar{p} K^{0}$, (b) $B^{+} \rightarrow p \bar{p} K^{+}$, (c) $B^{0} \rightarrow p \bar{p} K^{* 0}$, (d) $B^{+} \rightarrow p \bar{p} K^{*+}$ and (e) $B^{+} \rightarrow p \bar{p} \pi^{+}$modes. 


\subsubsection{Low $m_{p \bar{p}}$ enhancement}

The resulting sPlots for the $m_{p \bar{p}}$ distributions are shown in Figure 5.2 after removing $2.85<m_{p \bar{p}}<3.15 \mathrm{GeV} / c^{2}$. Although the $m_{p \bar{p}}$ enhancement at low mass is quite prominent in the $B^{+} \rightarrow p \bar{p} K^{+}, B^{0} \rightarrow p \bar{p} K^{0}$ and $B^{+} \rightarrow p \bar{p} \pi^{+}$modes, in the case of $B^{0} \rightarrow p \bar{p} K^{* 0}$ and $B^{+} \rightarrow p \bar{p} K^{*+}$ modes the statistics are too poor to draw a definite conclusion (as one can see from the distribution comparisons in Figure 5.3(a,b)). The shapes of the enhancement in $B^{0} \rightarrow p \bar{p} K^{0}$ and $B^{+} \rightarrow p \bar{p} K^{+}$are similar within the statistics of the measurements (see Figure 5.3(c)), in agreement with the theoretical predictions [122].

\subsubsection{Dalitz plot asymmetry}

To study the $m_{p h}$ and $m_{\bar{p} h}$ asymmetry, the Dalitz plot is divided along the diagonal $m_{p h}=m_{\bar{p} h}$ line and each of the two halves is projected onto the nearer axis. The corresponding sPlot distributions for the signal events in all the modes are shown in Figure 5.4. No asymmetry is expected to be introduced from variations in $\varepsilon_{m_{p \bar{p}}}$ which is charge-symmetric and slowly varying with $m_{p \bar{p}}$.

In $B^{+} \rightarrow p \bar{p} K^{+}$the asymmetry appears as a broad enhancement peaking at about $4 \mathrm{GeV}$ in the $p K^{+}$combinations (Figure 5.4(b)). This is contrary to the pole model predictions [122] of single $\Lambda_{b}$ pole dominance in $B^{+} \rightarrow p \bar{p} K^{+}$, that would lead to an $m_{\bar{p} K^{+}}$enhancement at high mass. It is also contrary to the flat distribution predicted by the form-factor model [28]. This feature could be an indication of a correlation between quarks in the $\bar{p}$ and the $K^{+}$if the $B$ decay proceeds through a penguin diagram (Figure 1.4(a)). No quantitative theoretical description of this correlation is available at present. The asymmetry in the low $m_{p \bar{p}}$ band in Figure 5.1(b) disfavors the possibility of the low mass $p \bar{p}$ enhancement originating only from the presence of a resonance below threshold (such as the baryonium candidate at $1835 \mathrm{MeV} / c^{2}$ recently seen by BES). ${ }^{1}$

In the case of $B^{0} \rightarrow p \bar{p} K^{0}$, there is no information on the flavor of $B$ (without tagging) and thus this study cannot be performed. For the $B^{0} \rightarrow p \bar{p} K^{* 0}$ and $B^{+} \rightarrow$

\footnotetext{
${ }^{1}$ This argument is similar to the one presented in Ref. [46] for gluonic state below threshold.
} 

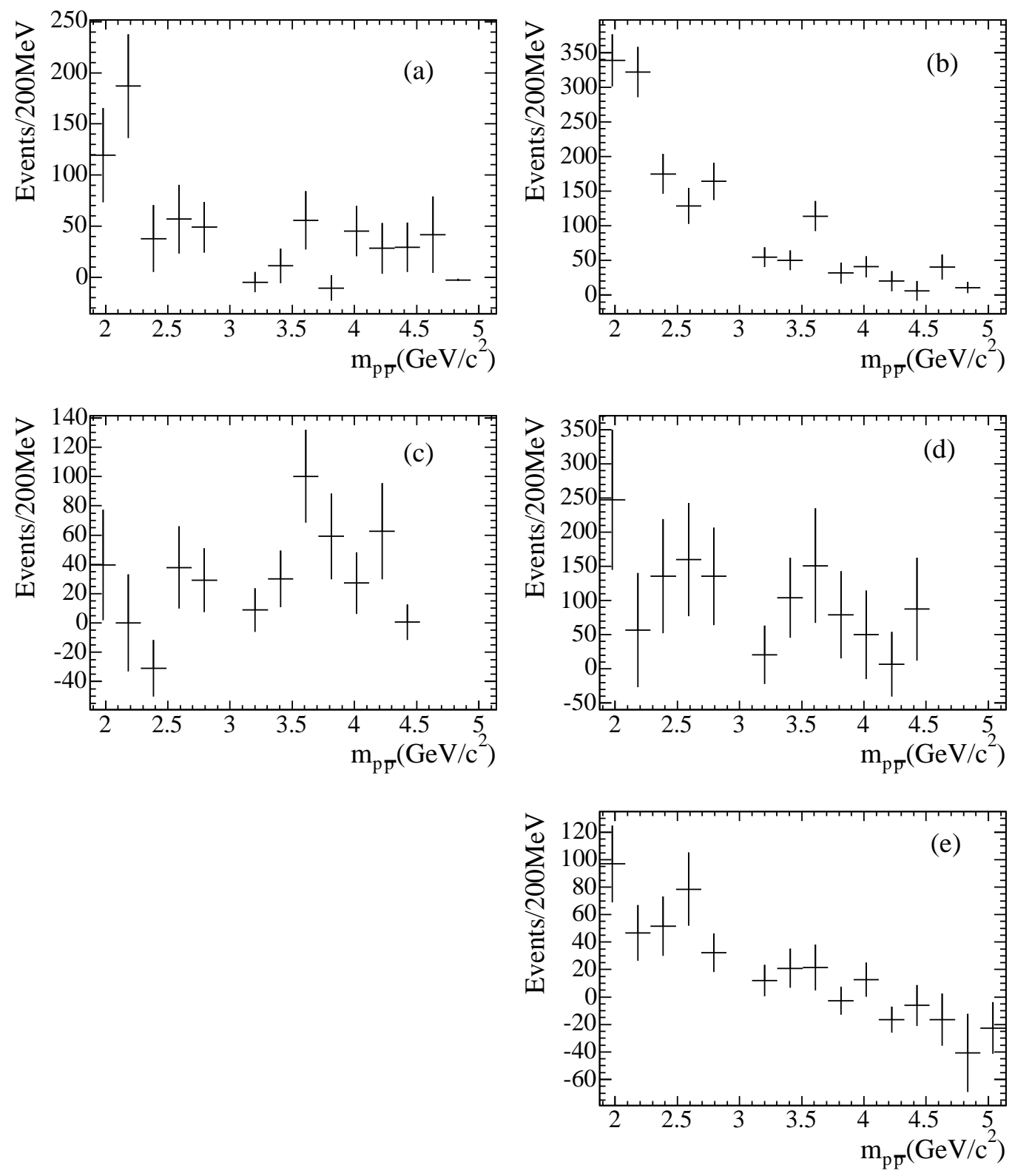

Figure 5.2: $m_{p \bar{p}}$ distribution sPlots for (a) $B^{0} \rightarrow p \bar{p} K^{0}$, (b) $B^{+} \rightarrow p \bar{p} K^{+}$, (c) $B^{0} \rightarrow p \bar{p} K^{* 0},(\mathrm{~d}) B^{+} \rightarrow p \bar{p} K^{*+}$ and (e) $B^{+} \rightarrow p \bar{p} \pi^{+}$modes removing $2.85<$ $m_{p \bar{p}}<3.15 \mathrm{GeV} / c^{2}$. 

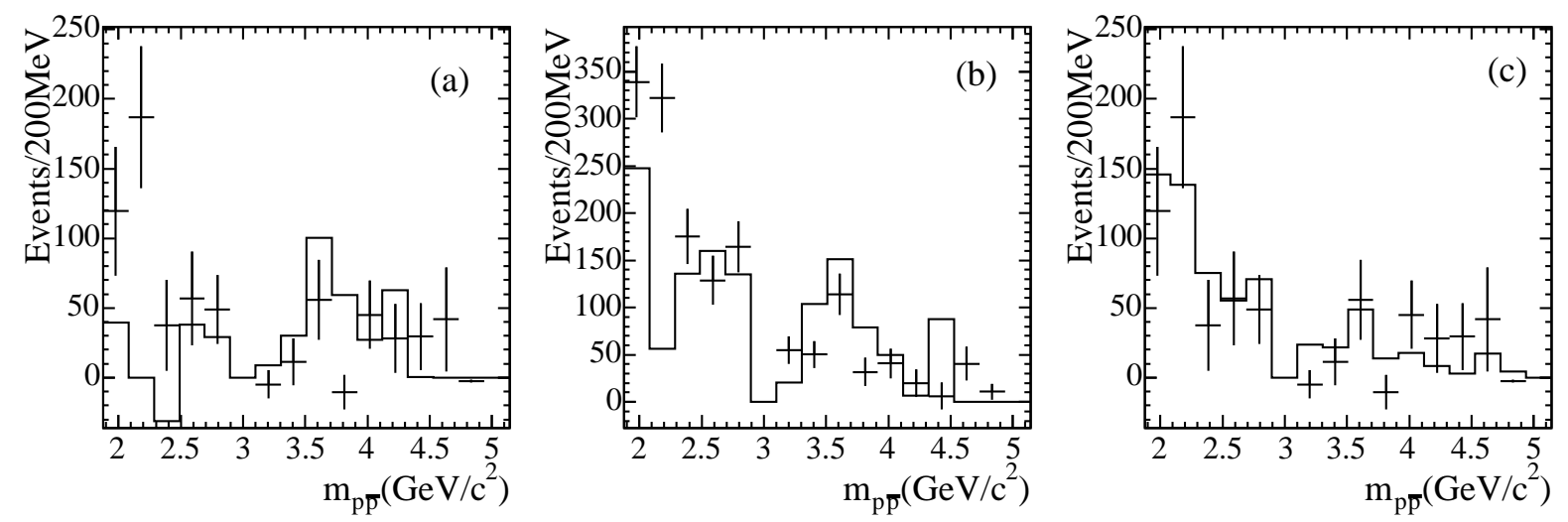

Figure 5.3: Comparisons between sPlots of $m_{p \bar{p}}$ distributions for (a) $B^{0} \rightarrow p \bar{p} K^{0}$ (errors) vs $B^{0} \rightarrow p \bar{p} K^{* 0}$ (histogram), (b) $B^{+} \rightarrow p \bar{p} K^{+}$(errors) vs $B^{+} \rightarrow p \bar{p} K^{*+}$ (histogram) and (c) rescaled to the same area $B^{+} \rightarrow p \bar{p} K^{+}$(histogram) vs $B^{0} \rightarrow$ $p \bar{p} K^{0}$ (errors) (removing $2.85<m_{p \bar{p}}<3.15 \mathrm{GeV} / c^{2}$ ).

$p \bar{p} K^{*+}$ modes there seems to be no difference between the two halves within the available statistics, while in the $B^{+} \rightarrow p \bar{p} \pi^{+}$mode, there is a marginal excess of events in the $m_{p \pi^{-}}$half of the Dalitz plot.

\subsection{Exotic searches}

We find no evidence for the presence of exotic resonances such as a glueball candidate $f_{J}(2330)$ or pentaquark candidates $\Theta^{+/ *++}$ in $B \rightarrow p \bar{p} h$ decays. The distribution of the signal events in $B^{+} \rightarrow p \bar{p} K^{+}$mode disfavors the presence of a resonance below threshold (such as the baryonium candidate at $1835 \mathrm{MeV} / \mathrm{c}^{2}$ recently seen by BES [39]).

\section{$5.4 \quad$ Future Prospects}

Three-body baryonic $B$ decays provide a unique laboratory to simultaneously study many different aspects of physics: hadron spectroscopy, $B$ physics, charmonium physics, studies of CP violation, tests of low-energy QCD, and of course, searches 

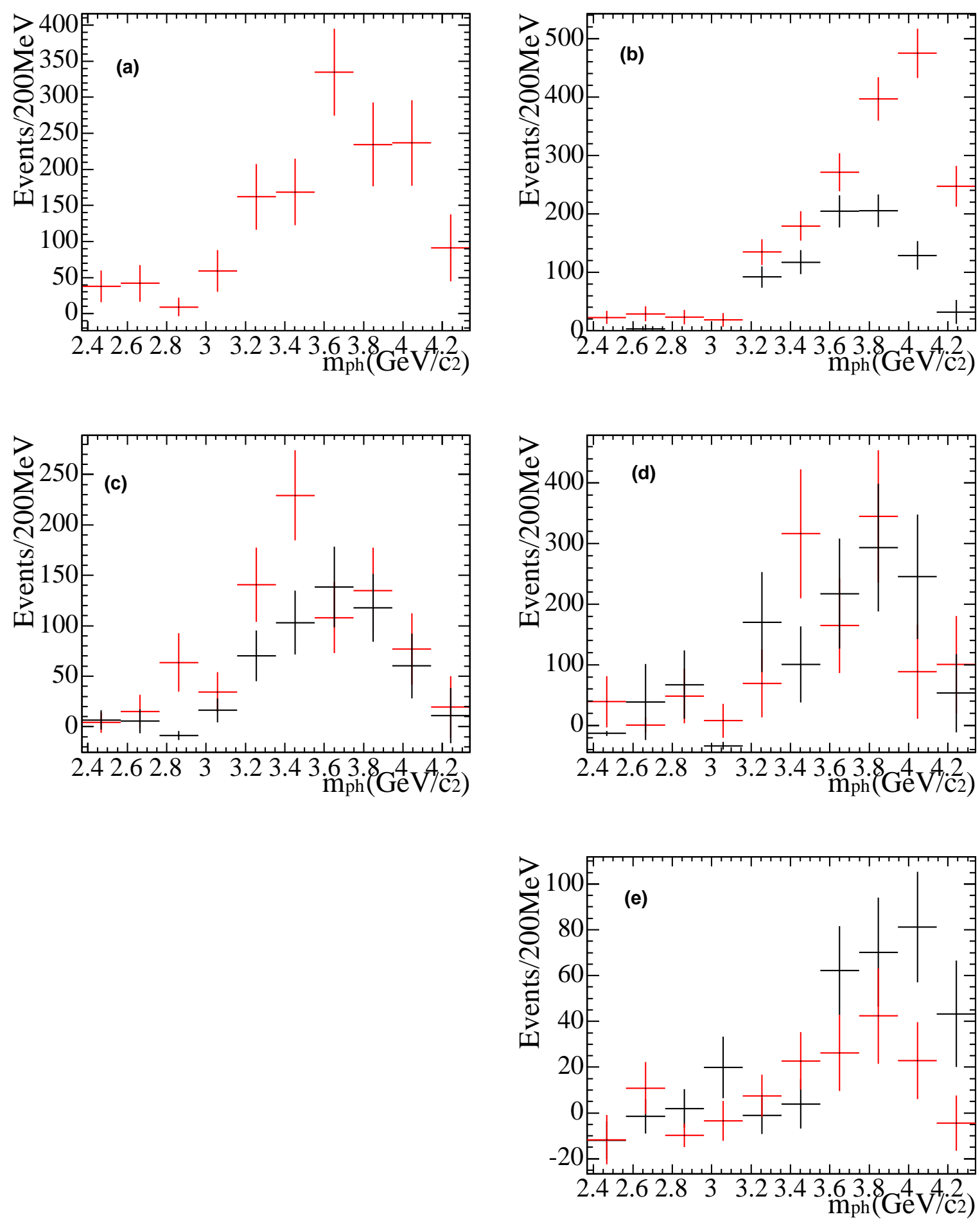

Figure 5.4: $m_{p h}$ distribution sPlots in red for $m_{p h}>m_{\bar{p} h}$ and in black for $m_{p h}<m_{\bar{p} h}$ for (a) $B^{0} \rightarrow p \bar{p} K^{0}$, (b) $B^{+} \rightarrow p \bar{p} K^{+}$, (c) $B^{0} \rightarrow p \bar{p} K^{* 0}$, (d) $B^{+} \rightarrow p \bar{p} K^{*+}$ and (e) $B^{+} \rightarrow p \bar{p} \pi^{+}$modes. 
for something unexpected.

A $50 \%$ increase in the BABAR dataset is expected by summer 2006 and a three-fold increase by end of 2008. This will allow more precise measurements of the branching fractions and the $m_{p \bar{p}}$ enhancement shapes, which in turn will lead to more stringent tests of theoretical models. New theoretical calculations of the expected $p \bar{p}$ angular distributions are anticipated [155].

It would be interesting to see if the $2 \sigma \mathrm{CP}$ asymmetry persists in the $B^{+} \rightarrow$ $p \bar{p} K^{+}$mode as the statistical errors decrease. A time-dependent analysis to study a $\mathrm{CP}$ asymmetry in the $B^{0} \rightarrow p \bar{p} K^{0}$ mode, a CP eigenstate, is currently starting. With more statistics, a time-dependent Dalitz plot analysis will also be achievable.

Larger data statistics will lead to tighter constraints on searches for exotics as well as higher potential for discovery of yet to be observed charmonium resonances (e.g. $\eta_{c}(2 S)$ in $m_{p \bar{p}}$ spectrum of $\left.B^{+} \rightarrow p \bar{p} K^{+}\right)$. With an increase in the data sample, it is possible to improve the limits on the branching fractions of decays of the type $B \rightarrow X_{c \bar{c}} h \rightarrow p \bar{p} h$, where $h=K^{+}, K_{S}^{0}, K^{* 0}$ and $K^{*+}$, and $X_{c \bar{c}}=\chi_{c 0}, \psi(3770)$ (or other known and new $c \bar{c}$ resonances).

A measurement of the spin of $\Lambda_{c}^{+}$in the decay $B \rightarrow \Lambda_{c}^{+} \bar{p}$ with $\Lambda_{c}^{+} \rightarrow p K_{S}^{0}$, can be used to verify the quark model predictions. It is also interesting to study the fully longitudinally polarized $J / \psi \rightarrow p \bar{p}$ helicity distribution produced in $B$ decays to test QCD calculations and to compare with similar measurements performed with $e^{+} e^{-} \rightarrow J / \psi$, where $J / \psi$ is transversely polarized [156].

With additional data, it will be possible to perform the measurements discussed above, so many more interesting and more precise results are soon to come. 


\section{Appendix A}

\section{Three-body decay kinematics}

\section{A.1 Phase-space}

Let us imagine that $B$ meson at rest decays into three particles. The partial decay rate of the $B$ meson of mass $m_{B}$ into three particles in its rest-frame is given in terms of the Lorentz-invariant matrix element $\mathcal{M}$ by

$$
d \Gamma=\frac{(2 \pi)^{4}}{2 m_{B}}|\mathcal{M}| d \Phi_{3}\left(\mathbf{p}_{\mathbf{B}} ; \mathbf{p}_{\mathbf{1}}, \mathbf{p}_{\mathbf{2}}, \mathbf{p}_{\mathbf{3}}\right)
$$

where $d \Phi_{3}$ is an element of three-body phase space given by

$$
\begin{aligned}
& d \Phi_{3}=\frac{d \mathbf{p}_{\mathbf{1}}}{E_{1}} \cdot \frac{d \mathbf{p}_{\mathbf{2}}}{E_{2}} \cdot \frac{d \mathbf{p}_{\mathbf{3}}}{E_{3}} \cdot \delta^{4}\left(\mathbf{p}_{\mathbf{B}}-\mathbf{p}_{\mathbf{1}}-\mathbf{p}_{\mathbf{2}}-\mathbf{p}_{\mathbf{3}}\right) \\
d \Phi_{3}= & \frac{d \mathbf{p}_{\mathbf{1}}}{E_{1}} \cdot \frac{d \mathbf{p}_{\mathbf{2}}}{E_{2}} \delta^{4}\left(\mathbf{p}_{\mathbf{1 2}}-\mathbf{p}_{\mathbf{1}}-\mathbf{p}_{\mathbf{2}}\right) \delta^{4}\left(\mathbf{p}_{\mathbf{B}}-\mathbf{p}_{\mathbf{1 2}}-\mathbf{p}_{\mathbf{3}}\right) d^{4} \mathbf{p}_{\mathbf{1 2}} \frac{d \mathbf{p}_{\mathbf{3}}}{E_{3}} \\
= & \frac{d \mathbf{p}_{\mathbf{1}}}{E_{1}} \cdot \frac{d \mathbf{p}_{\mathbf{2}}}{E_{2}} \delta^{4}\left(\mathbf{p}_{\mathbf{1 2}}-\mathbf{p}_{\mathbf{1}}-\mathbf{p}_{\mathbf{2}}\right) \cdot \frac{d \mathbf{p}_{\mathbf{3}}}{E_{3}} \\
= & \alpha \cdot \beta,
\end{aligned}
$$

where $\mathbf{p}_{\mathbf{1 2}}=\mathbf{p}_{\mathbf{B}}-\mathbf{p}_{\mathbf{3}}$. Then 


$$
\begin{aligned}
\alpha & \equiv \frac{d \mathbf{p}_{\mathbf{1}}}{E_{1}} \cdot \frac{d \mathbf{p}_{\mathbf{2}}}{E_{2}} \delta^{4}\left(\mathbf{p}_{\mathbf{1 2}}-\mathbf{p}_{\mathbf{1}}-\mathbf{p}_{\mathbf{2}}\right) \\
& =\frac{p_{1}^{*} d p_{1}^{*} d\left(\cos \theta_{1}^{*}\right) d \varphi_{1}^{*}}{E_{1} E_{2}} \delta\left(E-E_{1}-E_{2}\right),
\end{aligned}
$$

where $E=\sqrt{m_{1}^{2}+p_{1}^{* 2}}+\sqrt{m_{2}^{2}+p_{1}^{* 2}}$ in the $(1,2)$ rest frame.

$$
d E=\left(\frac{p_{1}^{*}}{E_{1}^{*}}+\frac{p_{1}^{*}}{E_{2}^{*}}\right) d p_{1}^{*}=\left(\frac{E_{1}^{*}+E_{2}^{*}}{E_{1}^{*} E_{2}^{*}}\right) p_{1}^{*} d p_{1}^{*}=\frac{m_{12}}{E_{1}^{*} E_{2}^{*}} p_{1}^{*} d p_{1}^{*} .
$$

Integrating Eq. A.4 over $\varphi_{1}^{*}$ and $E$ one obtains:

$$
\begin{gathered}
\alpha=2 \pi\left(\frac{p_{1}^{*}}{m_{12}}\right) d\left(\cos \theta_{1}^{*}\right) . \\
\beta=\frac{\mathbf{p}_{3}}{E_{3}}=\frac{p_{3}^{2} d p_{3} d \Omega_{3}}{E_{3}} .
\end{gathered}
$$

Integrating over $\Omega_{3}$ one obtains:

$$
\beta=\frac{4 \pi p_{3}^{2} d p_{3}}{E_{3}}
$$

In the overall center-of-mass frame:

$$
\begin{aligned}
p_{3}^{2} & =\frac{\left[s-m_{3}^{2}-m_{12}^{2}\right]^{2}-4 m_{3}^{2} m_{12}^{2}}{4 s} \\
E^{*} & =\sqrt{m_{3}^{@}+p_{3}^{2}}+\sqrt{m_{12}^{2}+p_{3}^{2}} .
\end{aligned}
$$

Then

$$
\begin{gathered}
d E^{*}=0=\frac{p_{3} d p_{3}}{E_{3}}+\frac{d\left(m_{12}^{2}\right)}{2 E_{12}}+\frac{p_{3} d p_{3}}{E_{12}} . \\
-p_{3} d p_{3} \frac{\left(E_{12}+E_{3}\right)}{E_{12} E_{3}}=\frac{d\left(m_{12}^{2}\right)}{2 E_{12}}
\end{gathered}
$$




$$
-\frac{p_{3}}{E_{3}} d p_{3}=\frac{d\left(m_{12}^{2}\right)}{2 \sqrt{s}}
$$

Thus

$$
\beta=\frac{2 \pi p_{3} d\left(m_{12}^{2}\right)}{\sqrt{s}}
$$

Substituting Eq. A.6 and Eq. A.13 into Eq. A.3:

$$
d \Phi_{3}=2 \pi\left(\frac{p_{1}^{*}}{m_{12}}\right) d\left(\cos \theta_{1}^{*}\right) 2 \pi \frac{p_{3}}{\sqrt{s}} d\left(m_{12}^{2}\right) .
$$

By definition of the phase-space the event population is flat in $\cos \theta_{1}^{*}$ thus

$$
\frac{d N_{p h s p}}{d\left(m_{12}^{2}\right)} \propto p_{1}^{*} p_{3}
$$

\section{A.2 Dalitz plot}

The scatter plot in $m_{12}^{2}$ and $m_{23}^{2}$ is called a Dalitz plot.

Considering this system quasi-two-body one can obtain from conservations of momentum and energy the following relations:

$$
\begin{aligned}
& \mathbf{p}_{\mathbf{1 2}}+\mathbf{p}_{\mathbf{3}}=0, \text { or } \\
& E_{12}^{2}-m_{12}^{2}=E_{3}^{2}-m_{3}^{2}
\end{aligned}
$$

and

$$
m_{B}=E_{12}+E_{3}
$$

Substituting expression for $E_{3}$ from A.17 one obtains:

$$
E_{3}=\frac{m_{B}^{2}-m_{12}^{2}+m_{3}^{2}}{2 m_{B}^{2}}
$$

by analogy one can obtain the expressions for $E_{2}$ and $E_{3}$. Summing over those three equations one can obtain another useful expression:

$$
m_{12}^{2}+m_{13}^{2}+m_{23}^{2}=m_{B}^{2}+m_{1}^{2}+m_{2}^{2}+m_{3}^{2} .
$$


The conservations of momentum and energy lead to the following relations:

$$
\mathrm{p}_{1}+\mathrm{p}_{2}+\mathrm{p}_{\mathbf{3}}=\mathbf{0}
$$

and

$$
m_{B}=E_{1}+E_{2}+E_{3} .
$$

From A.20 one obtains:

$$
\begin{aligned}
E_{3}^{2} & =m_{3}^{2}+\left(\mathbf{p}_{\mathbf{1}}+\mathbf{p}_{\mathbf{2}}\right)^{2}=m_{3}^{2}+2 p_{1} p_{2} \cos \theta+\mathbf{p}_{\mathbf{1}}^{2}+\mathbf{p}_{\mathbf{2}}^{2}, \\
& =m_{3}^{2}+2 \sqrt{E_{1}^{2}-m_{1}^{2}} \cdot \sqrt{E_{2}^{2}-m_{2}^{2}} \cos \theta+E_{1}^{2}-m_{1}^{2}+E_{2}^{2}-m_{2}^{2}
\end{aligned}
$$

and substituting expressions for $E_{1,2,3}$ from A.19:

$$
m_{B}^{2}-2 m_{b}\left(E_{1}+E_{2}\right)+2 E_{1} E_{2}-m_{3}^{2}+m_{1}^{2}+m_{2}^{2}+2 \sqrt{E_{1}^{2}-m_{1}^{2}} \cdot \sqrt{E_{2}^{2}-m_{2}^{2}} \cos \theta=0
$$

This equation gives the extent of the kinematic region, when $\cos \theta$ varies from +1 to -1 . The boundary of the Dalitz plot is obtained by setting $\cos \theta= \pm 1$, yielding a second order curve. 


\section{Appendix B}

\section{Study of Radiation Damage of CsI(Tl) Crystals}

\section{B.1 The Electromagnetic Calorimeter}

The electromagnetic calorimeter (EMC) is designed to measure electromagnetic showers with high efficiency, and provide excellent energy and angular resolution over the energy range from $20 \mathrm{MeV}$ to $9 \mathrm{GeV}$. EMC detects photons from $\pi^{0}$ and $\eta$ decays as well as from electromagnetic and radiative processes. The shape of the electromagnetic showers in the EMC also provides the primary source of information for electron identification.

\section{B.1.1 Geometry and Electronics}

As shown in Figure B.1, the EMC consists of a cylindrical barrel, containing 48 rings of 120 Thalium-doped Cesium Iodide $(\mathrm{CsI}(\mathrm{Tl}))$ crystals, and a conical endcap, containing rings of 120,100 , and $80 \mathrm{CsI}(\mathrm{Tl})$ crystals each. It has full azimuthal coverage and a polar angle acceptance extending from $15.8^{\circ}$ to $141.8^{\circ}$. The need for good energy and angular resolution dictated the choice of $\mathrm{CsI}(\mathrm{Tl})$, with its high light yield and small Molière radius; its short radiation length also allowed for a compact design. The fine segmentation provides the few mrad angular resolution needed to 


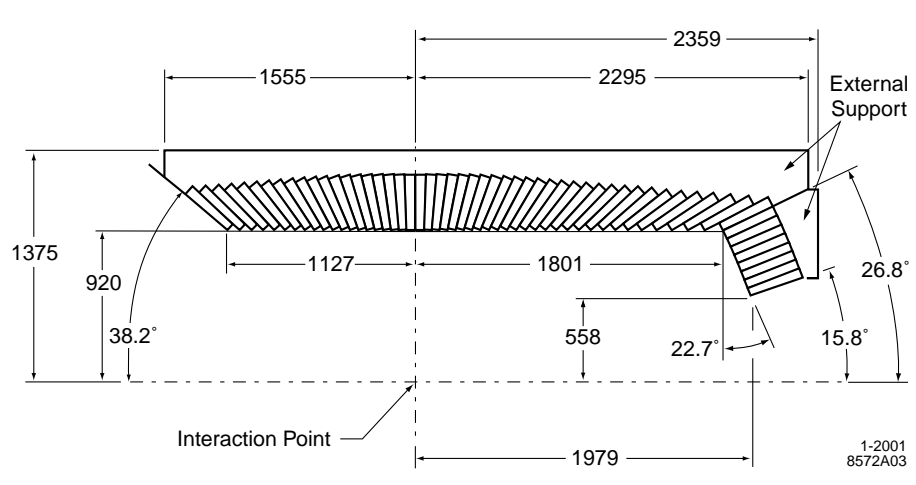

(a)

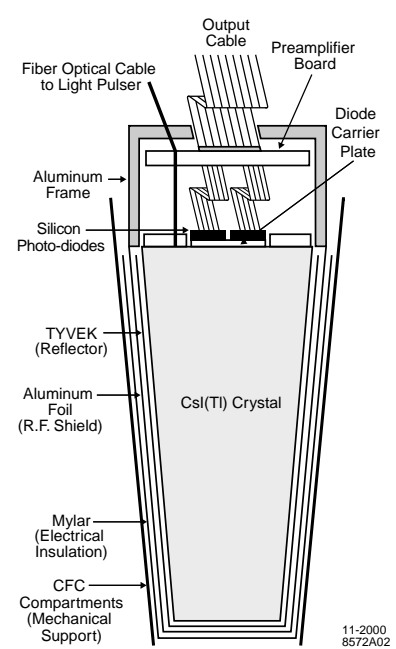

(b)

Figure B.1: (a) Longitudinal section of EMC. (b) Crystal housing with front-end electronics

achieve good $\pi^{0}$ mass resolution above $2 \mathrm{GeV}$.

As shown in Figure B.1, a pair of silicon photodiodes mounted at the end of each $\mathrm{CsI}(\mathrm{Tl})$ crystal registers the crystal light yield, and the signals are fed to a pair of low-noise preamplifiers. The amplified output is fed into the custom auto-range encoding (CARE) circuit; the total gain of the electronics chain is 256, 32, 4, or 1 for the four energy ranges 0-50 MeV, 50-400 MeV, 0.4-3.2 GeV, and 3.2-13.0 GeV. The two-fold redundancy of photodiodes and preamplifiers ensures reliability, since these components are inaccessible after completion of detector assembly.

\section{B.1.2 Reconstruction and Performance}

A typical electromagnetic shower spreads over a number of adjacent crystals, forming a cluster of energy deposits. Pattern recognition algorithms have been developed to not only identify these clusters, but to differentiate clusters with a single energy maximum from those with multiple energy maxima, referred to as bumps. A cluster is 
required to contain at least one seed crystal with an energy above $10 \mathrm{MeV}$. Surrounding crystals are included in the cluster if their energy exceeds a $1 \mathrm{MeV}$ threshold, or if they abut, in any direction, a crystal with at least $3 \mathrm{MeV}$ of energy. Clusters are split into as many bumps as there are local maxima, and an iterative algorithm is used to determine the bump energies.

At low energies (around 6.13 MeV), a radioactive source calibration measures the fractional EMC energy resolution to be $5.0 \pm 0.8 \%$; at higher energies (between 3 and $9 \mathrm{GeV}$ ) radiative Bhabha scattering events are used to determine the resolution to be $1.9 \pm 0.07 \%$. In the intermediate range (below $2 \mathrm{GeV}$ ), the energy resolution is inferred from the mass resolution of reconstructed $\pi^{0} \rightarrow 2 \gamma$ and $\eta \rightarrow 2 \gamma$ decays, with the two photons of approximately equal energies.

The energy resolution of the BaBar calorimeter extracted from a variety of processes - radioactive source, symmetric $\pi^{0}$ and $\eta$ decays, $\chi_{c 1} \rightarrow J / \psi \gamma$, and Bhabha events - is [157]:

$$
\frac{\sigma_{E}}{E}=\frac{(2.30 \pm 0.03 \pm 0.30) \%}{\sqrt[4]{E(G e V)}} \oplus(1.35 \pm 0.08 \pm 0.20) \%
$$

The first term comes from fluctuations in photon statistics, electronic noise and beam backgrounds; it is dominant at low energies. The constant term arises from non-uniformity in light collection, front and rear shower leakage and uncertainties in calibration; it dominates at high energies.

The angular resolution is determined solely from symmetric $\pi^{0}$ and $\eta$ decays. A fit to an empirical parametrization of the energy dependence gives:

$$
\sigma_{\theta}=\sigma_{\phi}=\left(\frac{3.87 \pm 0.07}{\sqrt{E(\mathrm{GeV})}} \oplus 0.00 \pm 0.04\right) \mathrm{mrad}
$$

which gives a resolution of about $12 \mathrm{mrad}$ at low energies and $3 \mathrm{mrad}$ at high energies. This slightly exceeds the performance predicted in simulation.

The reconstructed $\pi^{0}$ mass is measured to be $135.1 \mathrm{MeV}$ and is stable to better than a percent over the full photon energy range, with a width of $6.9 \mathrm{MeV}$. 


\section{B.2 Radiation Damage}

\section{B.2.1 Effects of Radiation on Crystals}

The crystals were produced[158] from a melt of CsI salt doped with $0.1 \%$ thalium using either Kyropoulos (Kharkov, Crismatec, Hilger) or Bridgman (Shanghai, Beijing) growth techniques. As sensitivity to radiation damage is generally found to be smaller for higher purity crystals, the quality of the salt and the recycled material was strictly controlled. In order to decrease the contributions to systematic errors on energy resolution it is important to understand the effect of radiation on $\mathrm{CsI}(\mathrm{Tl})$ crystals.

The total exposure of the EMC crystals is expected to reach up to $10 \mathrm{kRad}$ during the 10 year lifetime of the experiment. This integrated dose induces damage to the crystals, which may be exhibited in two ways: a drop in the light output and a change in the uniformity of the light output from energy deposition along the length of the crystal.

\section{B.2.2 Sources of Radiation Damage}

Radiation damage in the BABAR EMC is believed[159] to be almost entirely caused by 'non-physics' events, or so called 'beam backgrounds' in the EMC. There are two distinct types of this background in the BABAR experiment: single beam background and colliding beam background. The single beam background is mainly caused by fixed dipole magnets which are situated near the interaction point. They tend to sweep off-energy primary beam particles into machine elements near the detector, resulting in a low-energy shower $\left(E_{\gamma}<10 \mathrm{MeV}[136]\right)$ which enters the EMC. For colliding beams, there is also a major contribution of photons from small-angle radiative Bhabhas in which an $\mathrm{e}^{ \pm}$strikes a machine element. In both cases the occupancy increases significantly at small polar angles (in the endcap and backward barrel), while single beam backgrounds also peak in the horizontal plane. 

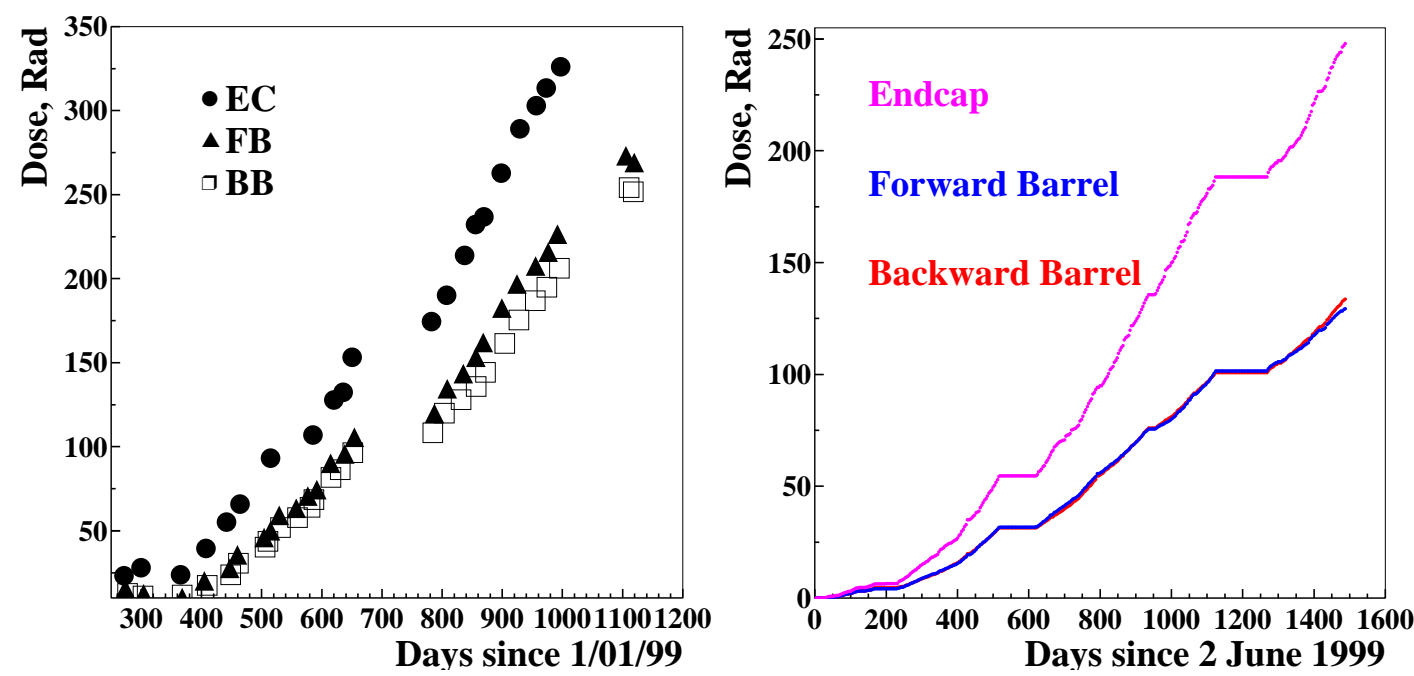

Figure B.2: Average dose in the EMC measured by (left) the RadFETs, (right) the leakage currents.

\section{B.3 Dose Monitoring}

\section{B.3.1 RadFET Monitoring}

The dose received by the front of the EMC is measured by 116 RadFETs[160] placed in front of the barrel and endcap crystals. RadFETs are real-time integrating dosimeters based on solid-state MOS technology. The dose increases approximately linearly with the integrated luminosity. The dose map obtained by the RadFETs reproduces the beam background angular distribution. The highest dose accumulated to date, $700 \mathrm{Rad}$, is observed in the innermost ring of the endcap (EC) while both backward (BB) and forward (FB) barrels have similar doses of about 250 Rad (Figure B.2(left)) on average.

\section{B.3.2 Leakage Currents}

An alternative way to calculate the dose accumulated by the crystals is using the leakage currents[161]. The dose then is proportional to the integral of beam-correlated 
photodiode current $(I)$ :

$$
\text { Dose }=\frac{E_{\text {crystal }}}{M_{\text {crystal }}}=\int \frac{I_{\text {beams on }}-I_{\text {beams off }}}{M_{\text {crystal }} \cdot C} d t
$$

where $M_{\text {crystal }}$ is the mass of crystals in the section of the detector and $C$ is the light output of 3900 photoelectrons/MeV obtained using the EMC readout (a light output of 7300 photoelectons/MeV was measured using a preamplifier with $2 \mu$ s shaping time). There are 10 independent bias voltage supplies for the EMC (four in the BB, four in the $\mathrm{FB}$ and two in the $\mathrm{EC}$ ). Using the formula above, one can obtain the average dose in each sector. The RadFETs measure the dose seen at the front face of the crystal. The leakage currents average the above dose over the whole crystal volume. They give similar results within a scaling factor of approximately 3 (Figure B.2(right)), which corresponds to the fraction of the crystal volume exposed to the radiation since the electro-magnetic showers deposit energy preferentially towards the front of the crystals. One could also investigate when most of the dose is deposited into the EMC: as shown in Figure B.3 the EMC exposures during stable beams and injection are approximately equal, while injection takes much shorter time. Thus dose rate during the injection is much higher.

\section{B.4 Total Light Yield (LY) Monitoring}

The observed integrated dose induces damage to the crystals, causing a drop in the total light output, hence result in a decrease of the photon statistics. The initial average light output of crystals was measured to be 3900 photoelectrons/MeV using standard EMC readout [161]. For the projected exposure over the lifetime of the BaBar experiment (10 kRad) the light yield drop would be $30 \%$ [162]. Thus its contribution towards degrading the energy resolution will be negligible compared to other contributions. We measure the change in the total light output using the standard BABAR calibration procedures[157]: radioactive source (6.13 MeV photons) and Bhabha events $(3-8.75 \mathrm{GeV}$ electrons). 

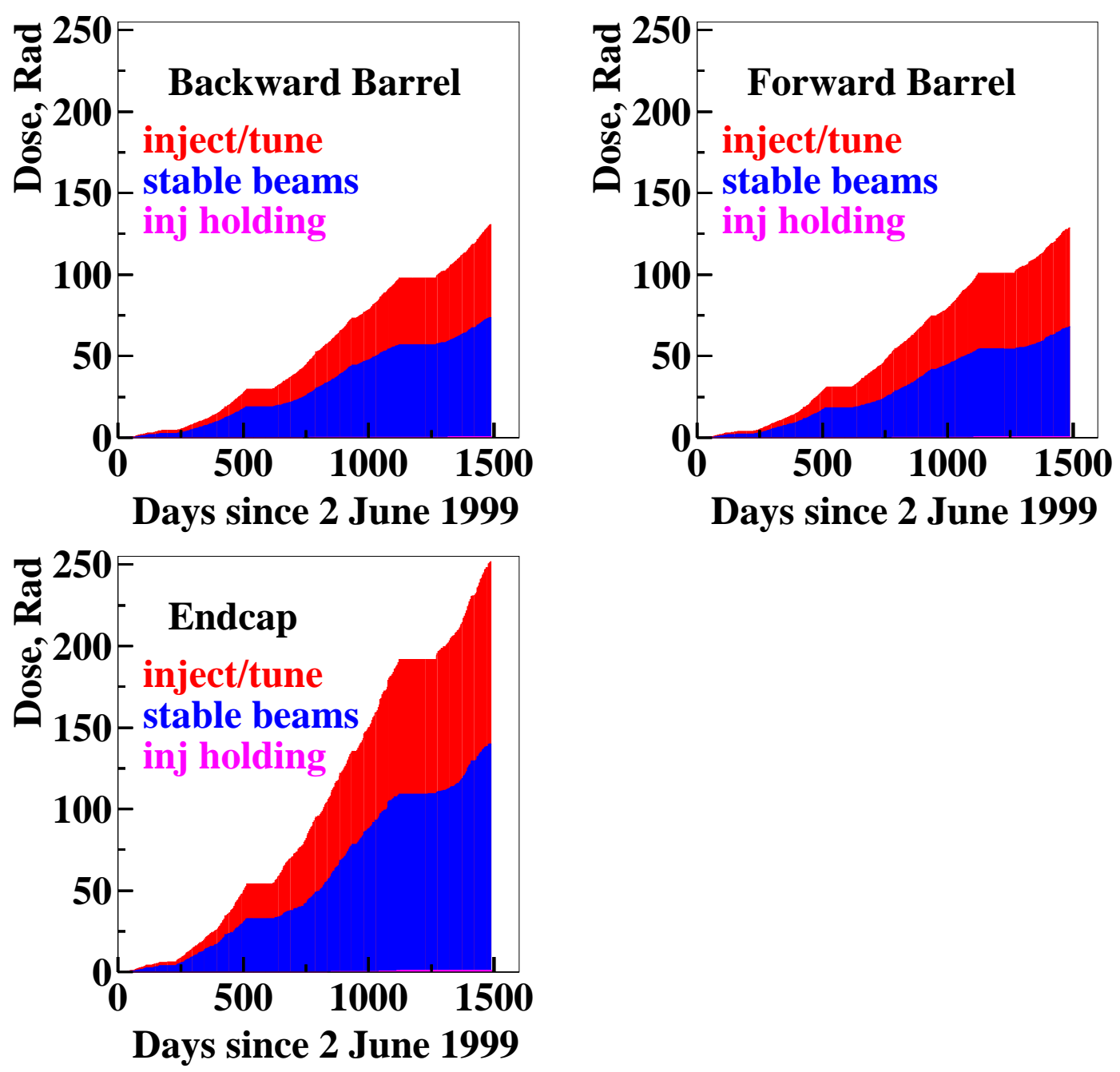

Figure B.3: Average dose in the EMC measured by the leakage currents depending on the beam condition. 

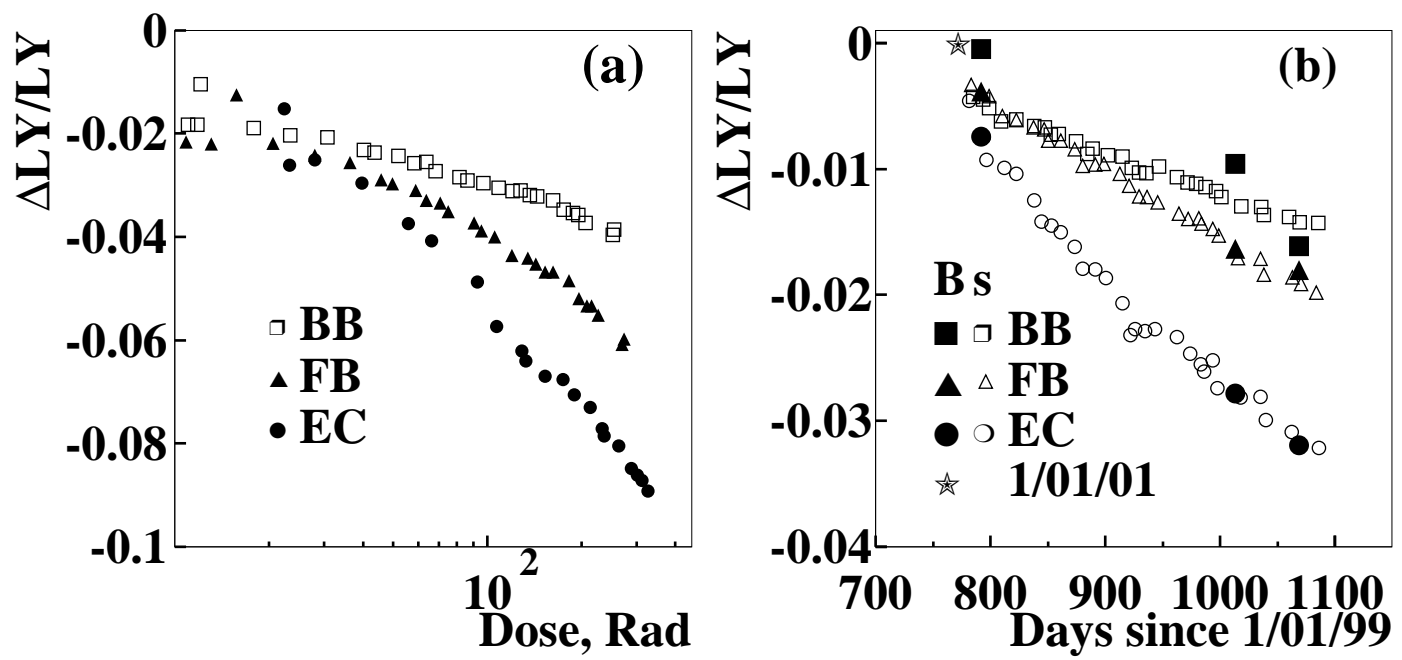

Figure B.4: Average change in the light yield in the EMC measured with (a) source (August 1999-December 2001), (b) source(s) and Bhabhas(B) (January-December 2001).

\section{B.4.1 Source Measurements}

We use 6.13 MeV photons from neutron-activated Fluorinert[163] circulating through a system of thin tubes in front of all crystals. These measurements are taken every 2 weeks and reach a precision of $0.33 \%$ for single crystals.

The dependence of the LY drop (averaged over EC, BB and FB) on the dose is presented in Figure B.4a. The value of the degradation is currently $9 \%$ in the EC, $6 \%$ in the $\mathrm{FB}$ and $3 \%$ in the BB. The LY decreases as a function of dose as expected, but the drop in LY differs for FB and BB, although they received similar doses as measured by the RadFETs.

To address this effect the LY change was studied separately for each crystal vendor (Figure B.5). Among crystals from the same vendor the values of the light yield degradation in the $\mathrm{FB}$ and the $\mathrm{BB}$ are similar. We are currently investigating the different rates of change of the LY in the barrel and the endcap. This may be explained by a significant portion of the EC crystals being irradiated both from the sides and from the front face, whereas the majority of the barrel crystals are irradiated from 
the front face only.
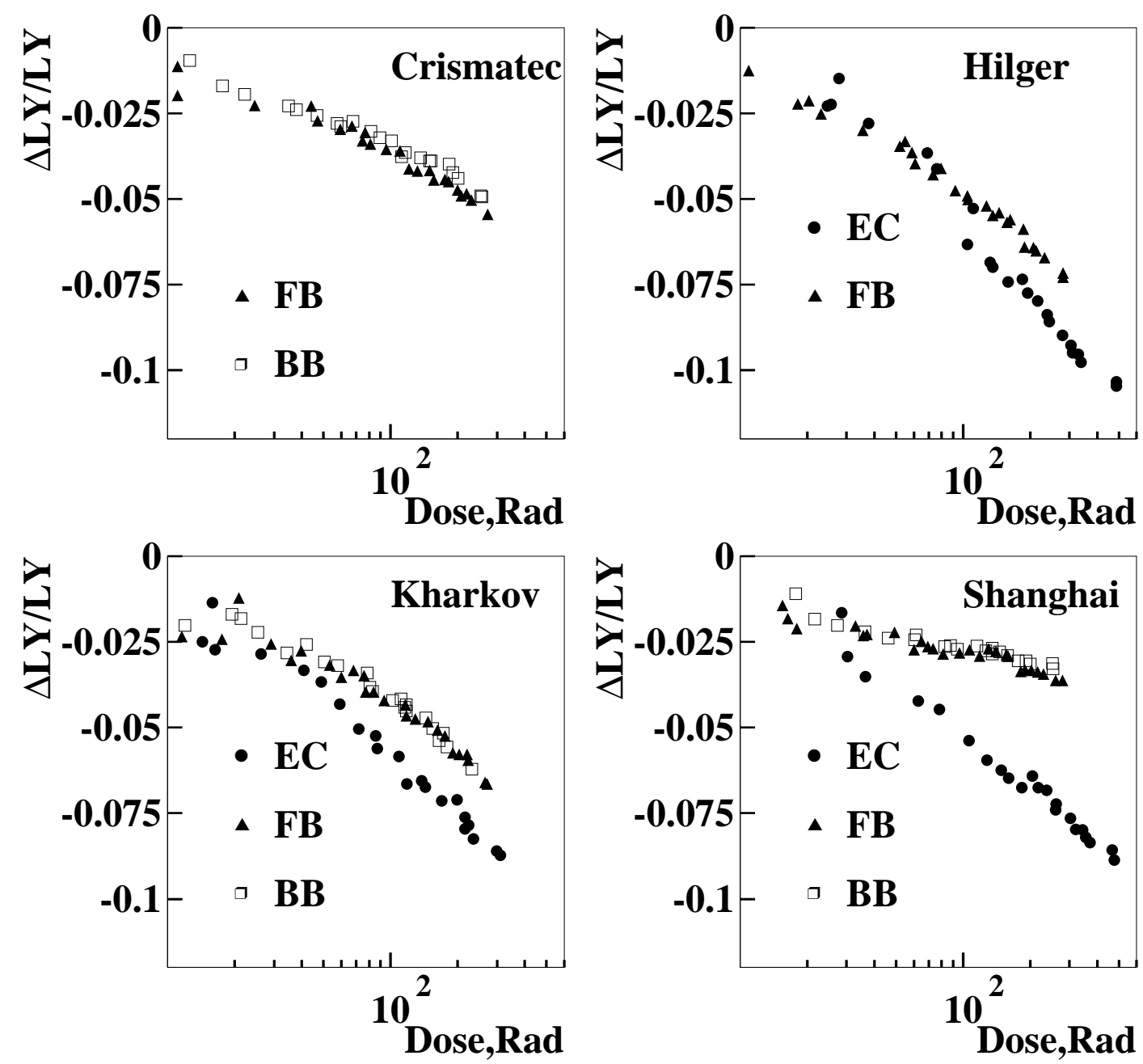

Figure B.5: Average change in the LY in the EMC by vendor.

\section{B.4.2 Bhabha Measurements}

Bhabha events allow the calibration of the calorimeter at high energies. In a 12hour run at a luminosity of $3 \times 10^{33} \mathrm{~cm}^{-2} \mathrm{~s}^{-1}$ we can reach $0.35 \%$ energy resolution per crystal. In the source measurements nearly all of the energy is deposited in the front part of the crystal, whereas in Bhabha events a large fraction of the electromagnetic shower is contained in the back part of the crystal which incidentally has less 
radiation damage. Currently we do not see any difference between the Bhabha and the source LY change measurements (Figure B.4b), thus there is as yet no evidence of non-uniformity.

\section{B.5 Study of Light-Yield Uniformity along the Crys- tal Length}

The scintillation light collection efficiency is not necessarily constant along the length of the crystal. This non-uniformity may result from variations in crystal clarity, surface finish and wrapping. The EMC crystals were wrapped with two layers of diffuse white reflector [164], each $165 \mu \mathrm{m}$ thick. The uniformity of light output along the wrapped crystal was measured by recording the signal from a highly collimated radioactive source at 20 points along the length of the crystal. The target for the nonuniformity contribution to the resolution of the EMC was less than $0.5 \%$. It led to the requirement for the light yield (LY) to be uniform within $\pm 2 \%$ in the front $10 \mathrm{~cm}$ of the crystal, the limit increasing linearly up to maximum of $\pm 5 \%$ at the rear face. Adjustments were made on individual crystals to meet these criteria by selectively roughing or polishing the crystal surface to reduce or increase reflectivity [165].

During the course of the BaBar experiment the EMC crystals accumulate a radiation dose caused primarily by low energy (up to $10 \mathrm{MeV}$ ) photons [159]. These photons deposit nearly all of their energy in the front third of the crystal, which may affect the uniformity of the light output along the length of the crystal. Previous studies [166] of the change in the longitudinal response by irradiation were inconclusive. To understand the impact of the radiation exposure we have constructed an apparatus that allows precise measurement of the longitudinal changes in light yield of large $\mathrm{CsI}(\mathrm{Tl})$ crystals. The systematic errors in these measurements are minimized by performing all the longitudinal scans completely in-situ, interleaved with $\mathrm{Co}^{60}$ exposures. 


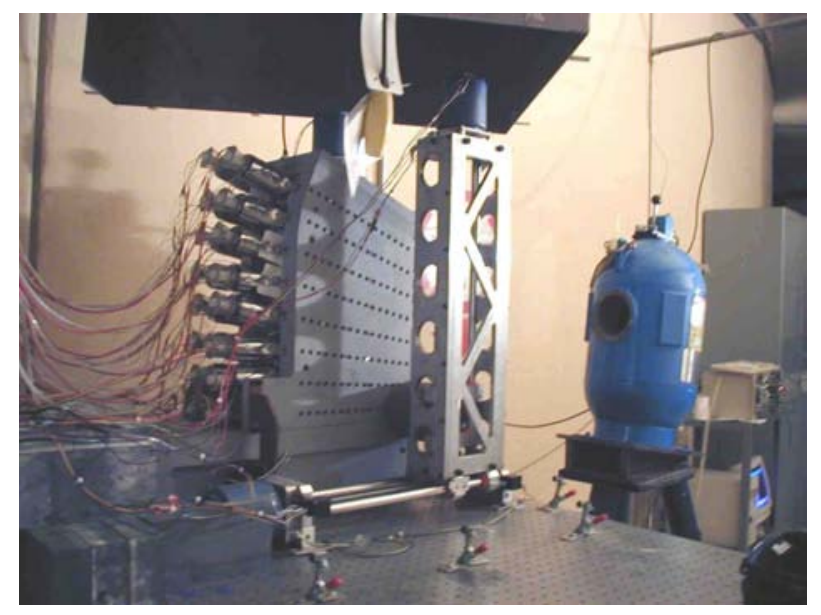

Figure B.6: Experimental Setup: crystal array on the left, ${ }^{60} \mathrm{Co}$ source on the right.

\section{B.5.1 Experimental Setup}

The apparatus is shown in Figure B.6. The assembly consists of $2 \times 8$ crystals $^{1}$ produced from a melt of CsI salt doped with $0.1 \%$ thallium, using either Kyropoulos (Type A) or Bridgman (Type B) growth techniques. The crystals are read out with Hamamatsu R2154-06 photomultiplier tubes (PMT). Four stepper motors move two $\mathrm{Pb}$ collimators with ${ }^{88} \mathrm{Y}$ sources in vertical and horizontal planes. The assembly is irradiated uniformly (maximum dose variations are less than 15\%) from the front face at a rate of $1-2 \mathrm{Rad} /$ hour by photons from a ${ }^{60} \mathrm{Co}$ source located $1 \mathrm{~m}$ from the assembly. The long outside surface of the crystals is shielded by a $5 \mathrm{~cm}$ steel plate with $0.91 \mathrm{~cm}$ holes drilled every $2 \mathrm{~cm}$ along each crystal length for collimation. The low dose rate and geometrical configuration were chosen to approximate radiation exposure of the crystals in the BaBar electromagnetic calorimeter, under typical beam conditions.

\footnotetext{
${ }^{1}$ Sixteen crystals studied in this experiment were part of the pool of "spares" which remained after the construction of the EMC. Out of those 16 crystals 4 Type A crystals were produced by Crismatec, Nemours, France and of Type B crystals 11 were made in Shanghai Institute of Ceramics, Shanghai, P.R. China and 1 in Beijing Glass Research Institute, Beijing, P.R. China. We have excluded 1 Shanghai and 1 Crismatec crystals from the current paper because of readout electronics problems. Another 2 Shanghai, 1 Crismatec and 1 Beijing crystals were excluded because we believe that they were not representative of the quality of the crystals installed in the EMC. Thus the results of this paper are based on 8 Shanghai and 2 Crismatec crystals.
} 
A small CsI $(\mathrm{Tl})$ crystal with PMT and ${ }^{88} \mathrm{Y},{ }^{22} \mathrm{Na}$ and ${ }^{228} \mathrm{Th}$ sources, located behind $10 \mathrm{~cm}$ of lead, is used as a standard reference to compensate for the drift of electronics. We use ten AD592s [167] for the temperature monitoring. The daynight temperature difference in the experimental room was less than $2^{\circ} \mathrm{C}$. A light pulser system with light fibers connected to the face of each crystal is used to monitor the electronics. Radiation monitoring is done with two GM tubes with a computer readout for the current dose monitoring and thermo-luminescent dosimeters for the total dose monitoring. Data is read out through a CAMAC crate/SCSI card to a PC.

\section{B.5.2 Measurements}

Thirty-five irradiations were made, increasing the exposure incrementally between each two irradiations. The total dose currently is $10 \mathrm{kRad}$. After waiting for 6 hours between exposures, data points were taken by moving the ${ }^{88} Y$ source in $2 \mathrm{~cm}$ steps along the length of each crystal.

The results of longitudinal scans at different doses are presented for typical crystals of Type A and B in Figure B.7(a) and B.8(a) respectively.

As we are interested only in the dose dependent contribution to the non-uniformity (Figure B.7(b) and B.8(b)) we can parametrize it as a linear function of position along the length of the crystal $(x)$ :

$$
\frac{L Y(x, D)}{L Y_{a v}(D)}-\frac{L Y(x, D=0)}{L Y_{a v}(D=0)}=\frac{\Delta_{r a d}(D)}{T}\left(\frac{T}{2}-x\right),
$$

where $\Delta_{\text {rad }}(D)$ is a percentage drop in the light yield from the back to the front of the crystal caused by irradiation, $L Y_{a v}$ is the light output averaged over all positions along the crystal length, $T$ is the length of the crystal, $x$ is the position along the crystal length, and $D$ is the dose.

The dose dependence of $\Delta_{\text {rad }}(D)$ is shown in Figure B.9. It can be parametrized as follows:

$$
\Delta_{\text {rad }}(D)=a \cdot \log _{10}^{2} D+b \cdot \log _{10} D+c
$$

The crystals can be subdivided into three categories: 


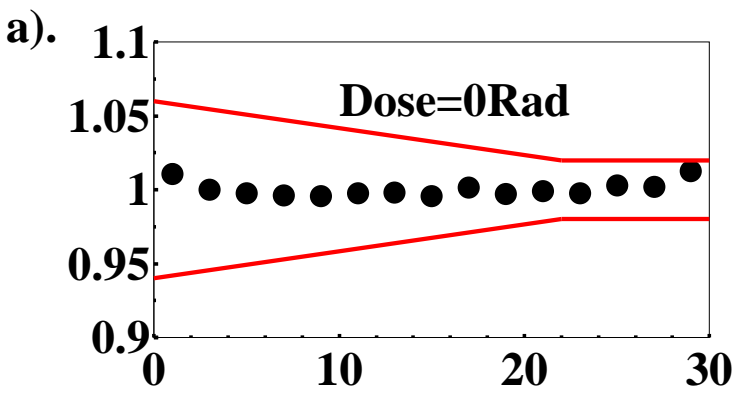

b). 0.04
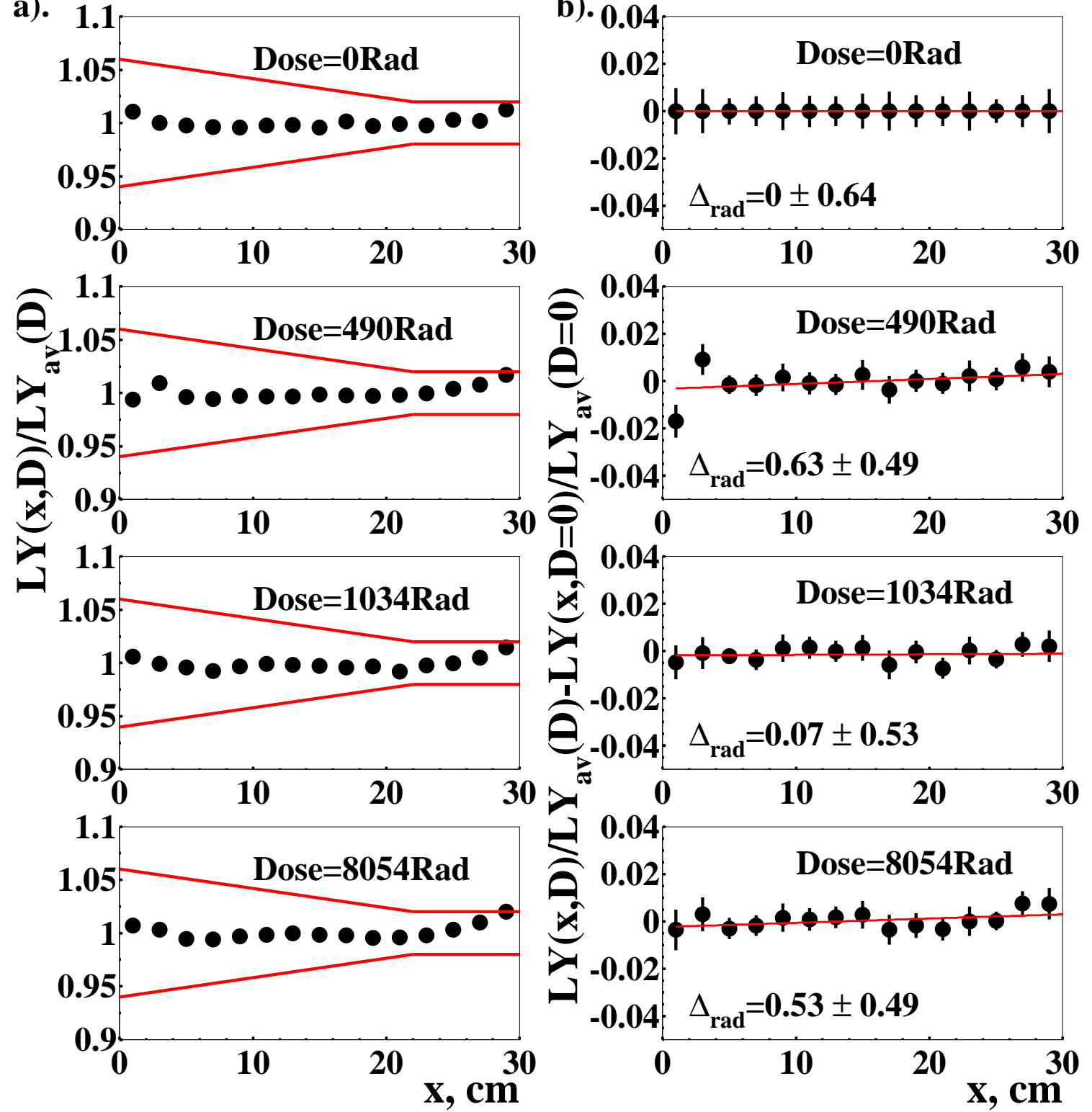

Figure B.7: a. Typical uniformity scan results for Type A crystal, solid line corresponds to the crystal uniformity requirement; b. Irradiation contribution to the uniformity, solid line corresponds to the fit results. Note: $\mathrm{x}=0$ is the back of the crystal. 


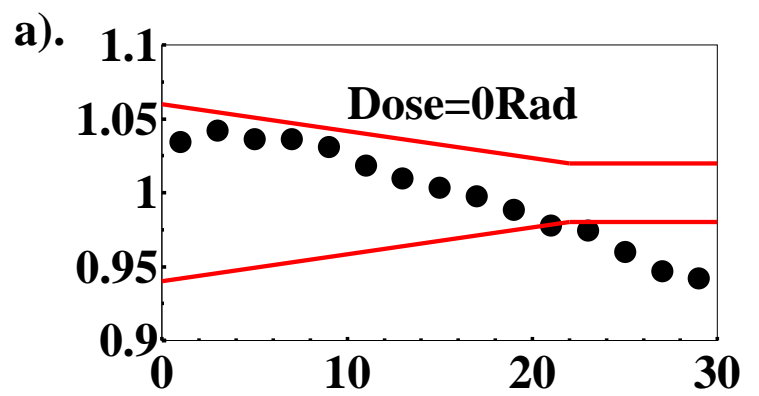

b).
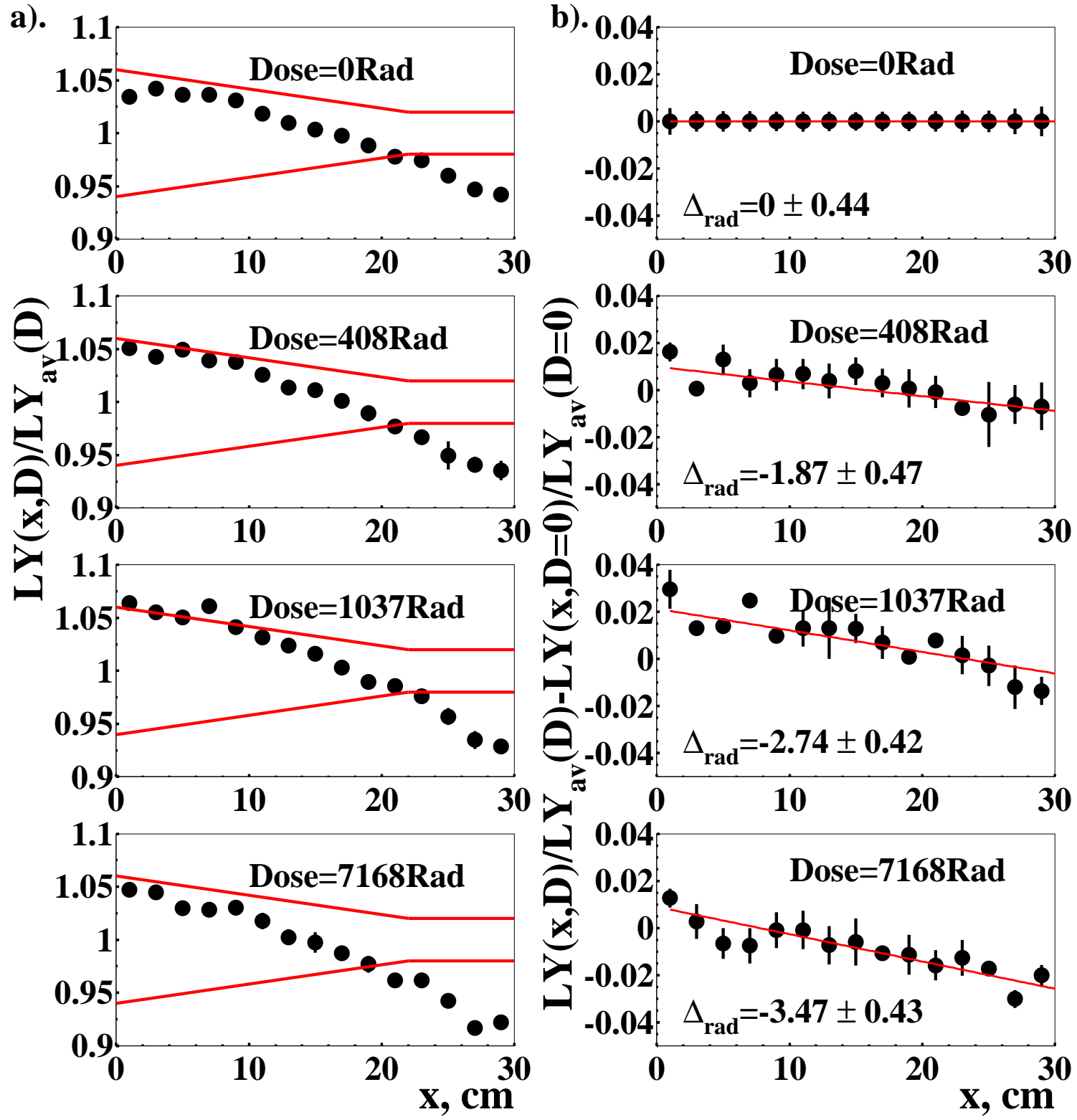

Figure B.8: a. Typical uniformity scan results for Type B crystal, solid line corresponds to the crystal uniformity requirement; b. Irradiation contribution to the uniformity, solid line corresponds to the fit results. Note: $\mathrm{x}=0$ is the back of the crystal. 
132 APPENDIX B. STUDY OF RADIATION DAMAGE OF CSI(TL) CRYSTALS

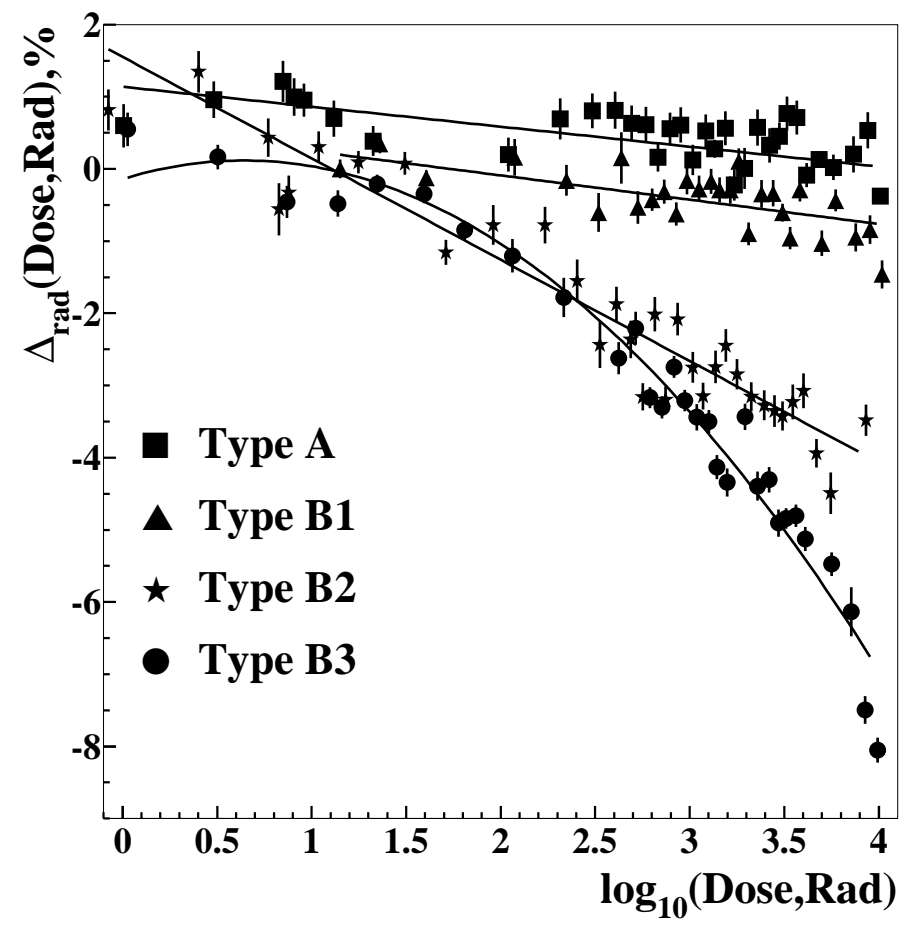

Figure B.9: Dose dependence of the percentage drop of the light yield for a sample of different crystal types. 
B.5. STUDY OF LIGHT-YIELD UNIFORMITY ALONG THE CRYSTAL LENGTH133

Table B.1: Parametrization of $\Delta(D)$ in percent

\begin{tabular}{|c||c|c|c|}
\hline & $\mathbf{a}$ & $\mathbf{b}$ & $\mathbf{c}$ \\
\hline \hline Type A & - & $-0.29 \pm 0.06$ & $0.88 \pm 0.17$ \\
\hline \hline Type B1 & - & $-0.43 \pm 0.06$ & $0.48 \pm 0.17$ \\
\hline Type B2 & - & $-1.39 \pm 0.06$ & $1.61 \pm 0.18$ \\
\hline Type B3 & $-0.68 \pm 0.05$ & $1.24 \pm 0.22$ & $-0.38 \pm 0.23$ \\
\hline \hline Type B & - & $-1.23 \pm 0.03$ & $1.43 \pm 0.09$ \\
\hline
\end{tabular}

- $b<-1$ : two crystals of Type A and three crystals of Type B (B1)

- $b>-1$ : three crystals of Type B (B2)

- $|a|>0$ : two crystals of Type B (B3)

Averaging the fits for 2 Type A and 8 Type $\mathrm{B}$ crystals we obtain values of $\mathrm{a}, \mathrm{b}$ and c for each of the types (see Table B.1). Using linear fit results only we estimate a light yield percentage drop at $10 \mathrm{kRad}$ of $\Delta_{\text {rad }}^{A}(10 \mathrm{kRad})=(-0.3 \pm 0.3) \%$ for Type A crystals and of $\Delta_{\text {rad }}^{B}(10 \mathrm{kRad})=(-3.5 \pm 0.2) \%$ for Type B crystals. The measured average light yield percentage drop at $10 \mathrm{kRad}$ is $(-0.4 \pm 0.5) \%$ and $(4.0 \pm 0.7) \%$ for crystals of Type A and Type B, respectively, which is in good agreement with the estimates $^{2}$.

\section{B.5.3 Study of the impact of non-uniformity}

We studied the effect of the light response uniformity on the energy resolution using the full BaBar GEANT 4 simulation without beam backgrounds. Single photons of $100 \mathrm{MeV}, 500 \mathrm{MeV}, 1 \mathrm{GeV}$ and $5 \mathrm{GeV}$ were produced at $|\cos \theta|<0.2$. Each crystal was divided into eight longitudinal slices. The non-uniformity was simulated as weights on the energy deposited in each slice.

The non-uniformity contribution to the energy resolution is shown in Figure B.10. From the measured energy resolution (Eq.B.1) we obtain the energy resolution for

\footnotetext{
${ }^{2}$ Previously [168] we reported the value of $\Delta_{\text {rad }}^{B}(10 \mathrm{kRad})=(-3.0 \pm 0.2) \%$, which is $1 \%$ higher than measured after extending dose to $10 \mathrm{kRad}$. This can be explained by the existence of Type B3 crystals with non-linear dose dependence of $\Delta_{\text {rad }}^{B}(D)$ which has not been established at $6 \mathrm{kRad}$.
} 


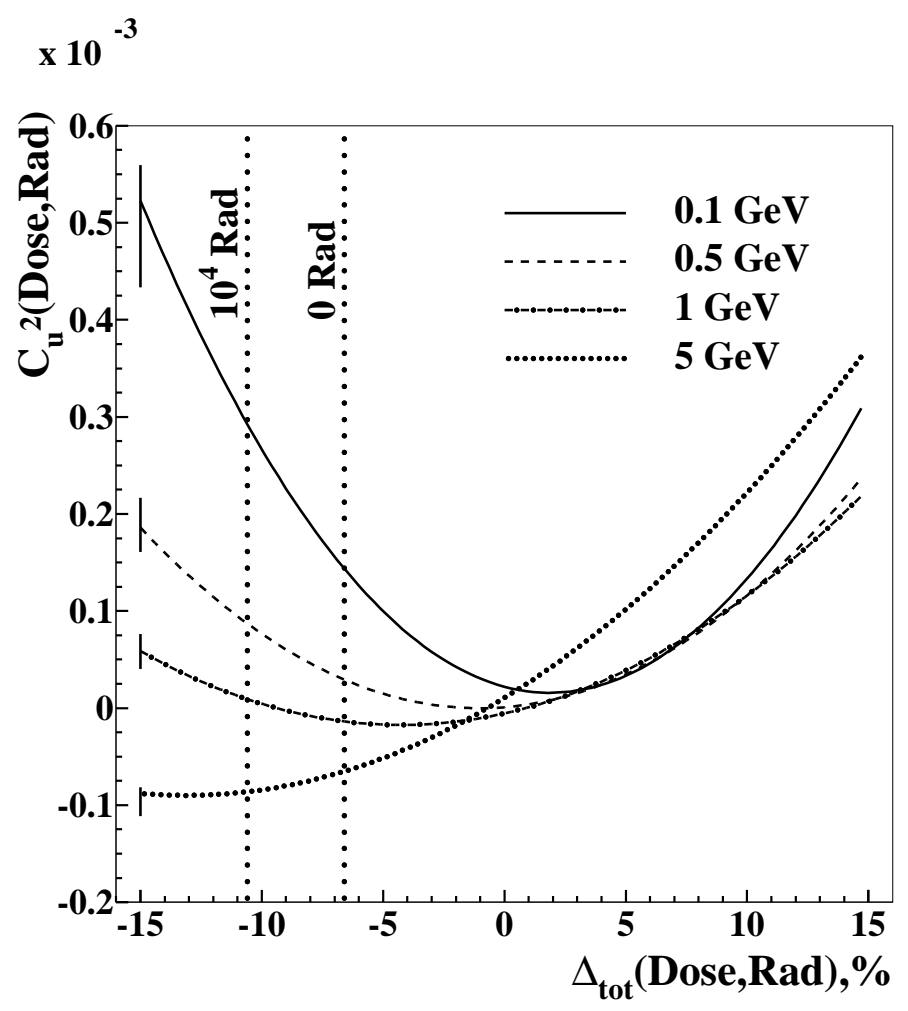

Figure B.10: MC study of the non-uniformity contribution to the energy resolution $\left(C_{u}\right)$ dependence on the total drop of the light yield $\left(\Delta_{t o t}\right)$. The error bars show typical uncertainties in the curves 
B.5. STUDY OF LIGHT-YIELD UNIFORMITY ALONG THE CRYSTAL LENGTH135

Table B.2: Estimate of the EMC resolution for single photons in \%

\begin{tabular}{|c||c|c|c|c|}
\hline & $0.1 \mathbf{G e V}$ & $0.5 \mathrm{GeV}$ & $1 \mathrm{GeV}$ & $5 \mathrm{GeV}$ \\
\hline \hline$\frac{\sigma_{E}}{E}(0 \mathbf{R a d})$ & $4.30 \pm 0.58$ & $3.05 \pm 0.42$ & $2.66 \pm 0.37$ & $2.05 \pm 0.29$ \\
\hline$C_{u}(0 \mathbf{R a d})$ & 1.19 & 0.54 & -0.37 & -0.81 \\
\hline$C_{u}(10 \mathbf{k R a d})$ & 1.71 & 0.93 & -0.30 & -0.93 \\
\hline \hline$\frac{\sigma_{E}}{E}(10 \mathbf{k R a d})$ & $\mathbf{4 . 4 7}$ & $\mathbf{3 . 1 4}$ & $\mathbf{2 . 6 7}$ & $\mathbf{2 . 0 0}$ \\
\hline
\end{tabular}

single photons of different energies, $\frac{\sigma_{E}}{E}$ (Table B.2, line 1). Knowing the percentage drop of the EMC crystals at zero dose, $\Delta_{0}=(-6.6 \pm 0.6) \%$ ([168]) one can estimate from Figure B.10 the initial non-uniformity contribution to the energy resolution, $C_{u}$ (Table B.2, line 2). Assuming the maximum predicted non-uniformity increase for $10 \mathrm{kRad}$, the total percentage drop in the light yield is $\Delta_{\text {tot }}(10 \mathrm{kRad})=\Delta_{0}+$ $\Delta_{\text {rad }}^{B}(10 \mathrm{kRad})=(-10.6 \pm 0.6) \%$. This allows us to estimate from Figure B.10 the nonuniformity contribution to the energy resolution at $10 \mathrm{kRad}, C_{u}(10 \mathrm{kRad})$ (Table B.2, line 3). Note that negative values of $C_{u}^{2}$ mean that sometimes non-uniformity improves the energy resolution. This is the case for photons with energies of a few $\mathrm{GeV}$ for which high values of the light yield at the back of the crystal compensate rear shower leakage. From the above considerations we predict the energy resolution at $10 \mathrm{kRad}$ from the initial energy resolution by subtracting the non-uniformity contribution at zero dose and adding the non-uniformity contribution at $10 \mathrm{kRad}$ in quadrature:

$$
\frac{\sigma_{E}}{E}(D)=\sqrt{\frac{\sigma_{E}^{2}}{E^{2}}(0)-\left( \pm C_{u}^{2}(0)\right)+\left( \pm C_{u}^{2}(D)\right)},
$$

where $\mathrm{D}$ is $10 \mathrm{kRad}$ and \pm corresponds to the sign of $C_{u}^{2}$. Comparing line 1 and line 4 in Table B.2, we conclude that the contribution of non-uniformity to the EMC resolution for doses up to $10 \mathrm{kRad}$ is negligible.

\section{B.5.4 Conclusion}

In this study we have measured the dose dependence of the drop in the light yield from the back to the front of the crystal to be $(-0.29 \pm 0.06) \cdot \log _{10} D+(0.88 \pm 0.17) \%$ 
136 APPENDIX B. STUDY OF RADIATION DAMAGE OF CSI(TL) CRYSTALS

for crystals grown by the Kyropoulos growth technique (Type A) and to be $(-1.23 \pm$ $0.04) \cdot \log _{10} D+(1.43 \pm 0.09) \%$ for crystals grown by the Bridgman growth technique (Type B). On the basis of this measurement we have been able to develop a correction function (Eq. B.4) to be used in Monte Carlo simulations to incorporate the effect of radiation damage on the crystal light yield uniformity. We estimate that even for the maximum observed uniformity decrease of $4 \%$ at $10 \mathrm{kRad}$, the EMC resolution will not be degraded significantly. 


\section{Bibliography}

[1] E. D. Bloom et al., Phys. Rev. Lett. 23, 930 (1969); M. Breidenbach et al., Phys. Rev. Lett. 23, 935 (1969).

[2] M. Gell-Mann, Phys. Lett 8, 214 (1964); G. Zweig, CERN-TH-401 and CERNTH-412 (1964), unpublished.

[3] V. E. Barnes et al., Phys. Rev. Lett. 12, 204 (1964).

[4] O. W. Greenberg, Phys. Rev. Lett. 13, 598 (1964).

[5] J. J. Aubert et al. [E598 Collaboration], Phys. Rev. Lett. 33, 1404 (1974); J. E. Augustin et al. [SLAC-SP-017 Collaboration], Phys. Rev. Lett. 33, 1406 (1974).

[6] H. Fritzsch, M. Gell-Mann and H. Leutwyler, Phys. Lett. B 47, 365 (1973).

[7] L. Lyons, P. F. Smith, G. J. Homer, J. D. Lewin, H. E. Walford and W. G. Jones, Z. Phys. C 36, 363 (1987); A. Marini et al., Phys. Rev. Lett. 48, 1649 (1982).

[8] L.B. Okun and M.B. Voloshin, JETP Lett. 23, 333(1976); A. De Rujula et al., Phys. Rev. Lett. 38, 317(1977);

[9] R. Jaffe, Phys. Rev. D 15, 267 and 281 (1977).

[10] J. Rosner, Phys. Rev. Lett. 21, 950 (1968).

[11] H. Fritzsch and M. Gell-Mann, Proceedings of the XVI Int. Conf. on HEP, J.D. Jackson and A. Roberts, eds. (National Accelerator Laboratory, Batavia, 1972), 
vol 2, p. 135; J. Kogut et al., Nucl. Phys. B 114, 199(1976); D. Robson, University of Liverpool preprint, LTH-35, 1977 (unpublished).

[12] D. Horn and J. Mandula, Caltech preprint CALT-68-575, 1977 (unpublished).

[13] W. Toki in Proceedings of the Summer Institute on Partile Physics 1996, SLAC, SLAC-R-58.

[14] L.G. Landsberg, Sov. Phys. Usp. 33, 169 (1990).

[15] C.J. Morningstar and M. Peardon, Phys. Rev. D 60034509 (1999).

[16] C. Michael, AIP Conf. Proc. 432, 657 (HADRON 97 Conf).

[17] S. Godfrey and N. Isgur, Phys. Rev. D 32, 189 (1985).

[18] J. Weinstein and N. Isgur, Phys. Rev. Lett. 48, 659 (1982); Phys. Rev. D 27, 588 (1983).

[19] R. Jaffe, Phys. Rev. D 17, 1444 (1978), R. Jaffe and K. Johnson, Phys. Lett. B 60, $201(1976)$.

[20] Particle Data Group, S. Eidelman et al., Phys. Lett. B 592, 1 (2004).

[21] R. M. Baltrusaitis et al. [MARK-III Collaboration], Phys. Rev. Lett. 56, 107 (1986).

[22] J. Z. Bai et al. [BES Collaboration], Phys. Rev. Lett. 76, 3502 (1996); J. Z. Bai et al. [BES Collaboration], Phys. Rev. Lett. 81, 1179 (1998).

[23] M. S. Alam et al. [CLEO Collaboration], Phys. Rev. Lett. 81, 3328 (1998);

R. Godang et al. [CLEO Collaboration], Phys. Rev. Lett. 79, 3829 (1997).

[24] J. E. Augustin et al. [DM2 Collaboration], Phys. Rev. Lett. 60, 2238 (1988).

[25] C. Amsler et al., Phys. Lett. B 520, 175 (2001).

[26] S. Godfrey and J. Napolitano, Rev. Mod. Phys. 71, 1411 (1999). 
[27] E.D. Bloom, SLAC-PUB-3573 (1985).

[28] C.K. Chua, W.S. Hou, S.Y. Tsai, Phys. Rev. D 66, 054004 (2002).

[29] E. Fermi and C. N. Yang, Phys. Rev. 76, 1739 (1949).

[30] Y. Nambu and G. Jona-Lasinio, Phys. Rev. 122, 345 (1961); Phys. Rev. 124, $246(1961)$.

[31] W. W. Buck, C. B. Dover and J. M. Richard, Annals Phys. 121, 47 (1979).

[32] E. Klempt, F. Bradamante, A. Martin and J. M. Richard, Phys. Rept. 368, 119 (2002).

[33] B. May et al. [ASTERIX Collaboration], Phys. Lett. B 225, 450 (1989).

[34] J.Z. Bai et al. [BES Collaboration], Phys. Rev. Lett. 91, 022001 (2003).

[35] A. Sibirtsev, J. Haidenbauer, S. Krewald, U. G. Meissner and A. W. Thomas, Phys. Rev. D 71, 054010 (2005).

[36] S. Jin for the BES Collaboration, Presented in Int. Conference on QCD and Hadronic Physics (QCD05), June 16-20, 2005, Beijing, China.

[37] A. Datta and P. J. O’Donnell, Phys. Lett. B 567, 273 (2003), G. J. Ding and M. L. Yan, Phys. Rev. C 72, 015208 (2005), M. L. Yan, S. Li, B. Wu and B. Q. Ma, arXiv:hep-ph/0405087, C. B. Dover and M. Goldhaber, Phys. Rev. D 15, 1997 (1977), I. S. Shapiro, Phys. Rept. 35, 129 (1978).

[38] C.-S. Gao, S.-L. Zhu, Commu. Theo. Phys. 42, 844 (2004).

[39] M. Ablikim et al. [BES Collaboration], Phys. Rev. Lett. 95, 262001 (2005).

[40] K. Abe et al. [Belle Collaboration], Phys. Rev. Lett. 88, 181803 (2002).

[41] K. Abe et al. [Belle Collaboration], Phys. Rev. Lett. 89, 151802 (2002); S. Anderson et al. [CLEO Collaboration], Phys. Rev. Lett. 86, 2732 (2001). 
[42] M. Ablikim et al. [BES Collaboration], Phys. Rev. Lett. 93, 112002 (2004).

[43] M. Z. Wang et al. [Belle Collaboration], Phys. Rev. Lett. 90, 201802 (2003); Y. J. Lee et al., Phys. Rev. Lett. 95, 061802 (2005).

[44] Y. J. Lee et al. [BELLE Collaboration], Phys. Rev. Lett. 93, 211801 (2004).

[45] C.K. Chua, W.S. Hou, S.Y. Tsai, Phys. Lett. B 544, 139 (2002).

[46] J.L. Rosner, Phys. Rev. D 68, 014004 (2003).

[47] B. Kerbikov, A. Stavinsky, V. Fedotov, Phys. Rev. C 69, 055205 (2004).

[48] D. V. Bugg, Phys. Lett. B 598, 8 (2004).

[49] G. Bardin et al., Nucl. Phys. B 411, 3 (1994), T. A. Armstrong et al. [E760 Collaboration], Phys. Rev. Lett. 70, 1212 (1993), M. Ambrogiani et al. [E835 Collaboration], Phys. Rev. D 60, 032002 (1999), M. Andreotti et al., Phys. Lett. B 559, 20 (2003).

[50] B. S. Zou and H. C. Chiang, Phys. Rev. D 69, 034004 (2004).

[51] G. S. Abrams et al., Phys. Rev. Lett. 33, 1453 (1974).

[52] W. Braunschweig et al. [DASP Collaboration], Phys. Lett. B 57, 407 (1975).

[53] N. Brambilla et al., CERN Yellow Report, CERN-2005-005 (2005). "Heavy quarkonium physics."

[54] E.J.Eichten and C.Quigg, Phys. Rev. D 49, 5845 (1994).

[55] L. P. Fulcher, Phys. Rev. D 44, 2079 (1991).

[56] S. N. Gupta and J. M. Johnson, Phys. Rev. D 53, 312 (1996).

[57] D. Ebert, R.N. Faustov and V.O. Galkin, Phys. Rev. D 67, 014027 (2003).

[58] J. Zeng, J. W. Van Orden and W. Roberts, Phys. Rev. D 52, 5229 (1995). 
[59] S. K. Choi et al. [BELLE collaboration], Phys. Rev. Lett. 89, 102001 (2002) [Erratum-ibid. 89, 129901 (2002)].

[60] K. Abe et al. [Belle Collaboration], Phys. Rev. Lett. 89, 142001 (2002).

[61] D.M. Asner et al. [CLEO Collaboration], Phys. Rev. Lett. 92, 142001 (2004).

[62] B. Aubert et al. [BABAR Collaboration], Phys. Rev. Lett. 92, 142002 (2004).

[63] J. L. Rosner et al. [CLEO Collaboration], Phys. Rev. Lett. 95, 102003 (2005).

[64] P. Rubin et al. [CLEO Collaboration], Phys. Rev. D. 72, 092004 (2005).

[65] M. Andreotti et al. [Fermilab E835 Collaboration], Phys. Rev. D 72, 032001 (2005).

[66] S. K. Choi et al. [Belle Collaboration], Phys. Rev. Lett. 91, 262001 (2003).

[67] B. Aubert et al. [BaBar Collaboration], Phys. Rev. D 71, 071103 (2005).

[68] D. Acosta et al. [CDF II Collaboration], Phys. Rev. Lett. 93, 072001 (2004).

[69] V. M. Abazov et al. [D0 Collaboration], Phys. Rev. Lett. 93, 162002 (2004).

[70] F. E. Close and P. R. Page, Phys. Lett. B 578, 119 (2004); N. A. Tornqvist, hep-ph/0308277; E. S. Swanson, Phys. Lett. B 588, 189 (2004); ibid. 598, 197 (2004).

[71] J. L. Rosner, Phys. Rev. D 70, 094023 (2004).

[72] K. Abe et al., Belle report BELLE-CONF-0541, hep-ex/0505038, paper no. LP2005-176, Lepton-Photon 2005.

[73] K. Abe et al., Belle report BELLE-CONF-0540, hep-ex/0505037, paper no. LP2005-175, Lepton-Photon 2005.

[74] S.K. Choi et al. [Belle Collaboration], Phys. Rev. Lett. 94, 182002 (2005).

[75] J.L. Rosner, hep-ph/0508155, Presented at PIC2005. 
[76] D. Cronin-Hennessy et al. [CLEO Collaboration], Phys. Rev. Lett. 92, 222002 (2004).

[77] P. Pakhlov [Belle Collaboration], hep-ex/0412041, presented at 32nd International Conference on High Energy Physics, Beijing, Proceedings, vol. 1, pp. 636639.

[78] K. Abe et al., Belle report BELLE-CONF-0576, hep-ex/0507033. Presented at PIC2005.

[79] B. Aubert et al., Phys. Rev. Lett. 95, 142001 (2005).

[80] F. J. Llanes-Estrada, Phys. Rev. D 72, 031503 (2005).

[81] S. L. Zhu, Phys. Lett. B 625212 (2005); E. Kou and O. Pene, Phys. Lett. B 631164 (2005); F. E. Close and P. R. Page, Phys. Lett. B 628, 215 (2005).

[82] L. Maiani, V. Riquer, F. Piccinini and A. D. Polosa, Phys. Rev. D 72, 031502 (2005).

[83] A. R. Dzierba, C. A. Meyer and A. P. Szczepaniak, J. Phys. Conf. Ser. 9, 192 (2005).

[84] R. A. Schumacher, nucl-ex/0512042.

[85] A. J. G. Hey and R. L. Kelly, Phys. Rept. 96, 71 (1983).

[86] D. Diakonov, V. Petrov and M. Polyakov, Z. Phys. A 359, 305 (1997).

[87] T. Nakano et al., [LEPS Collaboration] Phys. Rev. Lett. 91, 012002 (2003).

[88] T. Nakano, 2004 Presented at QNP2004 www.qnp2004.org

[89] T. Stepanyan et al. [CLAS Collaboration], Phys. Rev. Lett. 91, 252001 (2003).

[90] V. Kubarovsky et al. [CLAS Collaboration], Phys. Rev. Lett. 92, 032001 (2004).

[91] J. Barth et al. [SAPHIR Collaboration], Phys. Lett. B 572, 127 (2003). 
[92] M. Abdel-Bary et al. [COSY Collaboration], Phys. Lett. B 595, 127 (2004).

[93] P. Aslanyan, V. Emelyanenko and G.G. Rikhkvitzkaya, Nucl. Phys. A 755, 375 (2005).

[94] A. Aleev et al. [SVD Collaboration], Phys. Atom. Nucl. 68, 974 (2005).

[95] V. V. Barmin et al. [DIANA Collaboration], Phys. Atom. Nucl. 66, 1715 (2003).

[96] A.E. Asratyan, A.G. Dolgolenko and M.A. Kubantsev, Phys. Atom. Nucl. 67, $682(2004)$.

[97] A. Airapetian et al. [Hermes Collaboration], Phys. Lett. B 585, 213 (2004).

[98] S. Chekanov et al. [ZEUS Collaboration], Phys. Lett. B 591, 7 (2004).

[99] C. Alt et al. [NA49 Collaboration], Phys. Rev. Lett. 92, 042003 (2004).

[100] A. Aktas et al. [H1 Collaboration], Phys. Lett. B 588, 17 (2004).

[101] H. Z. Huang, nucl-ex/0509037.

[102] N. Baltzell et al., to be submitted to Phys Rev. Lett.; reported by V. Burkert at the Lepton Photon Conference, Uppsala, Sweden, July, 2005.

[103] M. Battaglieri et al. [CLAS Collaboration], accepted Phys. Rev. Lett., Dec 2005, hep-ex/0510061.

[104] A. Aleev et al. [SVD Collaboration], hep-ex/0509033.

[105] M. I. Adamovich et al. [WA89 Collaboration], Phys. Rev. C 72, 055201 (2005).

[106] Y. M. Antipov et al. [SPHINX Collaboration], Eur. Phys. J. A 21, 455 (2004).

[107] M. J. Longo et al. [HyperCP Collaboration], Phys. Rev. D 70, 111101 (2004).

[108] I. Abt et al. [HERA-B Collaboration], Phys. Rev. Lett. 93, 212003 (2004).

[109] K. Mizuk et al. [Belle Collaboration], Phys. Lett. B 632, 173 (2006). 
[110] J. Coleman, A Search for Pentaquarks at BaBar, Ph.D. Thesis, University of Liverpool (May 2005); BABAR -THESIS-05/015; K. Götzen, hep-ex/0510041, presented in QCD05, Montpellier, France, 4-8 Jul 2005.

[111] C. Risler, presented at XIII International Workshop on Deep Inelastic Scattering, Wisconsin, U.S.A.

[112] B. Aubert et al.[BABAR Collaboration], Phys. Rev. Lett. 95, 042002 (2005).

[113] J. Z. Bai et al. [BES Collaboration], Phys. Rev. D 70, 012004 (2004).

[114] D. O. Litvintsev [CDF Collaboration], Nucl. Phys. Proc. Suppl. 142, 374 (2005).

[115] S. Schael et al. [ALEPH], Phys. Lett. B 599, 1 (2004).

[116] J. Ellis, M. Karliner, M. Praszalowicz, JHEP 0405, 002 (2004); B. Wu, B.Q. Ma, Phys. Rev. D 69, 077501 (2004); H. Walliser, V.B. Kopeliovich, J. Exp. Theor. Phys. 97, 433 (2003); D. Borisyuk, M. Faber, A. Kobushkin, Ukr. J. Phys. 49, 944 (2004).

[117] J.L. Rosner, Phys. Rev. D 69, 094014 (2004).

[118] H. Albrecht et al. [ARGUS Collaboration], Phys. Lett. B 209, 119 (1988).

[119] N. Paver and R. Riazuddin, Phys. Lett. B 201, 279 (1988).

[120] V. Chernyak and I. Zhitnitsky, Nucl. Phys. B 345, 137 (1990).

[121] M. Jarfi et al., Phys. Rev. D 43, 1599 (1991); Phys. Lett. B 237, 513 (1990).

[122] H.Y. Cheng, K.C. Yang, Phys. Rev. D 66, 014020(2002).

[123] P. Ball and H.G. Dosch, Z. Phys. C 51, 445 (1991).

[124] N. Gabyshev et al. [Belle Collaboration], Phys. Rev. D 66, 091102 (2002); R. Chistov et al. [Belle Collaboration], hep-ex/0510074.

[125] N. Gabyshev et al. [Belle Collaboration], Phys. Rev. Lett. 90, 121802 (2003); 
[126] I. Dunietz, Phys. Rev. D 58, 094010 (1998).

[127] W. S. Hou and A. Soni, Phys. Rev. Lett. 86, 4247 (2001).

[128] J. Schumann, hep-ex/0505098.

[129] S. J. Brodsky and S. Gardner, Phys. Rev. D 65, 054016 (2002).

[130] N. Cabibbo, Phys. Rev. Lett. 10, 531 (1963); M. Kobayashi and T. Maskawa, Prog. Th. Phys. 49, 652 (1973).

[131] M.Z. Wang et al. [Belle Collaboration], Phys. Rev. Lett. 92, 131801 (2004).

[132] M. Z. Wang et al. [Belle Collaboration], Phys. Lett. B 617, 141 (2005).

[133] S. J. Brodsky and G. R. Farrar, Phys. Rev. Lett. 31, 1153 (1973), S. J. Brodsky and G. R. Farrar, Phys. Rev. D 11, 1309 (1975).

[134] B. Aubert et al. [BABAR Collaboration], Phys. Rev. Lett. 93131801 (2004).

[135] B. Aubert et al. [BABAR Collaboration], Phys. Rev. Lett. 94181802 (2005).

[136] B. Aubert et al. [BABAR Collaboration], Nucl. Instrum. Meth. A 479, 1 (2002).

[137] C. Hearty BABAR Analysis Document 135 (2001); http://babarhn.slac.stanford.edu:5090/HyperNews/get/physAnal/1727.html.

[138] S. Agostinelli et al., Nucl. Instrum. Meth. A 506, 250 (2003).

[139] The BABAR Physics Book, SLAC-R-504, Table 3-1.

[140] http://www.slac.stanford.edu/BFROOT/www/Physics/Tools/Pid/Hadrons/ Description_of_the_LH_selectors.html

[141] H. Albrecht et al. [ARGUS Collaboration], Phys. Lett. B 241, 278 (1990).

[142] R.A. Fisher, Ann. Eugenics 7, 179 (1936).

[143] R. Aleksan, et al. BABAR Analysis Document 350; A. Gaidot, et al. BABAR Analysis Document 461. 
[144] N. Berger, BABAR Analysis Document 300.

[145] B. Aubert et al. [BABAR Collaboration], Phys. Rev. D 73, 012005 (2006).

[146] R. Barlow, Computer Physics Communications 149, 97 (2002).

[147] http://babar-hn.slac.stanford.edu:5090/HyperNews/get/physAnal/1727.html.

[148] http://www.slac.stanford.edu/BFROOT/www/Physics/Tools/Vertex/cascade/ VtxCascadeNote.pdf.

[149] http://hfag.phys.ntu.edu.tw/hfagc/current/00105.html (November 04 2005).

[150] M. Pivk, F. Le Diberder, Nucl. Instr. Meth. A 555, 356 (2005).

[151] T. C. Petersen, M. H. Schune BABAR Analysis Document 630, Sec. 5.4.

[152] E. Varnes et.al. BABAR Analysis Document 157 (2001).

[153] R. Faccini et.al. BABAR Analysis Document 19 (2000).

[154] B. Aubert et al. [BABAR Collaboration], Phys. Rev. D 72072003 (2005).

[155] Chun-Khiang Chua, private communications.

[156] K. Abe et al. [The Belle Collaboration], hep-ex/0509020.

[157] M. Kocian, "X ${ }^{\text {th }}$ Int. Conf. on Calorimetry in Particle Physics", World Scientific, 2002, pp.167-174.

[158] Amcrys-H, Kharkov, Ukraine; Crismatec, Nemours, France; Hilger Analytical, Margate, Kent, UK; Shanghai Institute of Ceramics, Shanghai, P.R. China; Beijing Glass Research Institute, Beijing, P.R.China.

[159] S.H. Robertson, private communications.

[160] B. Camanzi et.al., Nucl.Instr.Meth. A 457, 476 (2001).

[161] I. Eschrich, "X $\mathrm{X}^{\text {th }}$ Int. Conf. on Calorimetry in Particle Physics", World Scientific, 2002 . 
[162] J. Bauer, IEEE Trans. Nucl. Sci. 51, 1596 (2004); T. Hryn'ova, in Proc. "Calorimetry in Particle Physics", 2002, pp.175-181.

[163] Fluorinert([C2F3CI]) is manufactured by 3M Corporation, St. Paul, MN, USA.

[164] TYVEK, registered trademark of E.I. DuPont de Nemours \& Co., Wilmington, DE, USA.

[165] P.M. Vavra, NSS 1998 Conference Record, IEEE, 1998, vol.1, pp. 525-530.

[166] R.Y. Zhu, "IV th Int. Conf. on Calorimetry in HEP", Frascati Physics Series, vol. VI, 1996, pp.589-598; J. Allison et al., NSS 1998 Conference Record, IEEE, 1999, vol.1, pp. 6-13; P. Vavra, private communications.

[167] AD592 is a two terminal monolithic integrated circuit temperature transducer produced by Analog Devices, USA.

[168] T. Hryn'ova et al., NSS 2003 Conference Record, IEEE, 2004, vol. 1, pp. 219222. 